\title{
Organocatalytic Asymmetric Arylation of $p$-Quinone Phosphonates: A Green Access to Biaryl Monophosphorus Ligands
}

\author{
Guodong Sun ${ }^{\mathrm{a}, \mathrm{b}}$, Zhuofei Deng', Zhonghua Luo ${ }^{\mathrm{c}}$, Zhongqing Wang ${ }^{\mathrm{c}, *}$ and Jiancun Zhanga,* \\ a State Key laboratory of Respiratory Diseases, Guangzhou Institutes of Biomedicine and Heath, \\ Chinese Academy of Sciences, 190 Kaiyuan Road, Guangzhou, 510530, P. R. China \\ ${ }^{\mathrm{b}}$ University of Chinese Academy of Sciences, No. 19 Yuquan Road, Beijing, 100049, P. R. China \\ c State Key Laboratory of Anti-Infective Drug Development, Sunshine Lake Pharma Co., Ltd, \\ Dongguan 523871, P. R. China
}

\section{Supporting Information}

Table of Contents

1、 Table S1-Optimization of the Reaction Conditions of $4 \mathrm{aa}^{a}$

2、Table S2-Optimization of the Reaction Conditions with quinone $2 \mathrm{~b}$ as the model substrate a

3、Table S3-Substrates scope of quinones with different phosphonate esters ${ }^{\mathrm{a}}$.....................5

4、 Table S4-Substrates scope of 2-naphthols and phenols ${ }^{\mathrm{a}}$.............................................5

5. General procedure for asymmetric synthesis of 4 aa ...........................................6

6、 General procedure for asymmetric synthesis of chiral biaryl phosphonates ....................7

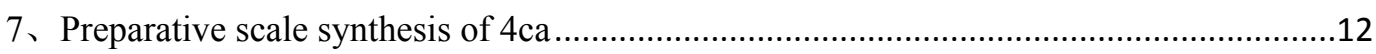

8. Preparation of chiral phosphine 6 from $4 \mathrm{ca}$..............................................................13

9、Typical Experimental Procedure for the Synthesis of Compounds 7a and 7b...............14

10、 Typical Experimental Procedure for the Synthesis of Compound 8...........................15

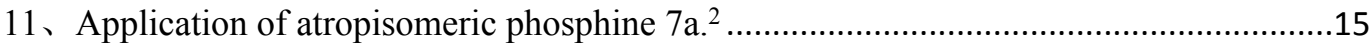

12、X-ray Single Crystal Structure Analysis of $4 \mathrm{ca}$.......................................................16

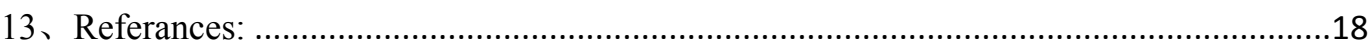

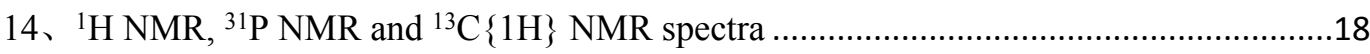

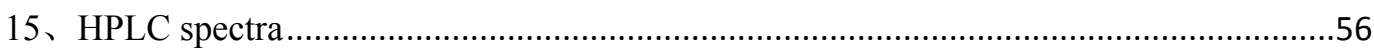




\section{General information}

If no special treatments are indicated, all reagents and solvents were used without further purification. NMR spectra were measured on a Bruker Avance 400 spectrometer or 600 spectrometer in solvents indicated; Chemical shifts were recorded in ppm $(\delta)$, and the residual solvent peak was used as an internal reference: proton (chloroform $\delta$ 7.26, DMSO $\delta 2.50$ ), carbon (chloroform $\delta$ 77.0, DMSO $\delta$ 39.5). Coupling constants are reported in $\mathrm{Hz}$ with multiplicities denoted as singlet (s), doublet (d), triplet (t), quartet (q), dd (doublet of doublets); m (multiplets), and etc. HRMS were performed on Fourier Transform Ion Cyclotron Resonance Mass Spectrometer. Analytical HPLC for liquid phase was carried out on an Agilent HPLC workstation. Melting points were measured using a melting point instrument and are uncorrected. Analytical thin layer chromatography TLC was performed on Huanghai HSGF 254 silica gel plates $(0.2 \pm$ $0.03 \mathrm{~mm}$ thick) with fluorescent indicator $254 \mathrm{~nm}$. Silica gel $60 \mathrm{H}$ (200-300 mesh), manufactured by Qingdao Haiyang Chemical Group Co. (China), was used for general chromatography. Optical rotations were measured using a $1 \mathrm{~mL}$ cell with a $1 \mathrm{dm}$ path length on a Jasco P-1030 polarimeter and are reported as follows: $[\alpha]_{\mathrm{D}}^{20}$ (c in g per $100 \mathrm{~mL}$ solvent). Racemic products were obtained by using diphenyl phosphate as the catalyst.

\section{1、Table S1-Optimization of the Reaction Conditions of $4 \mathbf{a a}^{a}$}

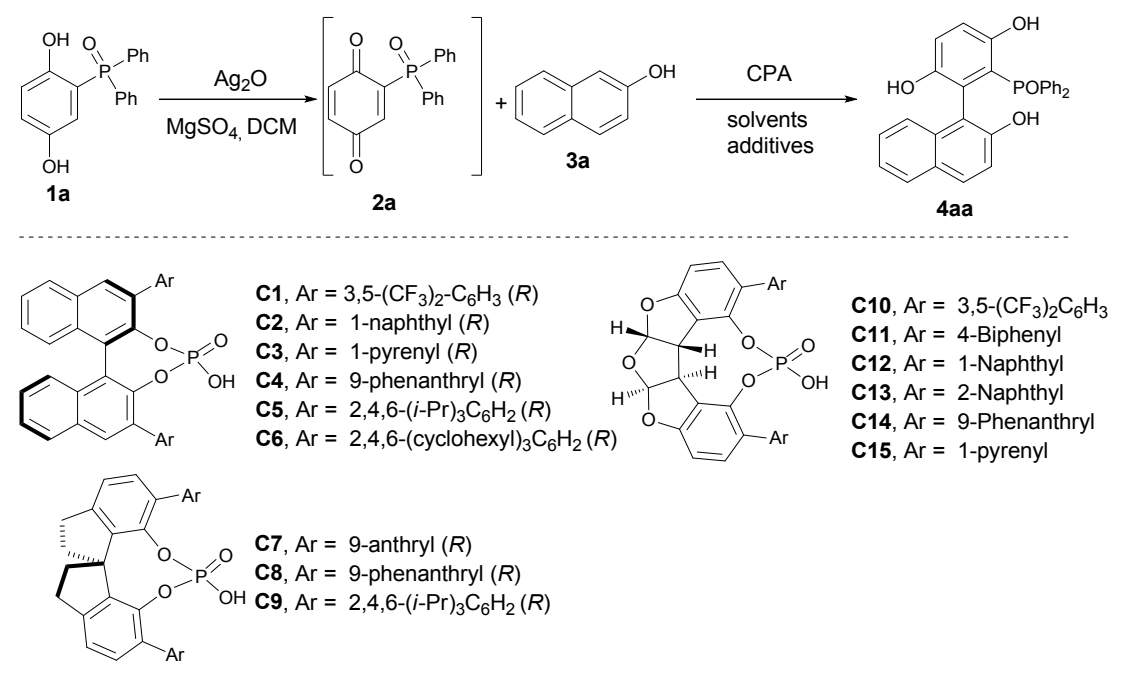

\begin{tabular}{cccccc}
\hline Entry & catalyst & solvent & Time $(\mathrm{h})$ & ${\text { Yield }(\%)^{b}}^{b}$ & ee $(\%)^{c}$ \\
\hline 1 & C1 & DCM & 29 & 67 & 0 \\
2 & C2 & DCM & 23 & 67 & -5 \\
3 & C3 & DCM & 29 & 68 & 3.5 \\
4 & C4 & DCM & 23 & 65 & 0 \\
5 & C5 & DCM & 48 & 41 & -2.4 \\
6 & C6 & DCM & 48 & 51 & -3.2 \\
7 & C7 & DCM & 29 & 56 & 12.1 \\
8 & C8 & DCM & 23 & 65 & 8.2 \\
9 & C9 & DCM & 48 & 50 & 2.2 \\
\hline
\end{tabular}




\begin{tabular}{cccccc}
\hline 10 & C10 & DCM & 30 & 52 & 3.9 \\
11 & C11 & DCM & 30 & 54 & 3.7 \\
12 & C12 & DCM & 24 & 56 & 6.2 \\
13 & C13 & DCM & 24 & 56 & 2.3 \\
14 & C14 & DCM & 24 & 53 & 0 \\
15 & C15 & DCM & 24 & 54 & 3.5 \\
$18^{d}$ & C7 & DCM & 24 & 26 & 9.3 \\
$19^{e}$ & C7 & DCM & 24 & 9 & 12.1 \\
$20^{f}$ & C7 & DCM & 24 & 48 & 12.6 \\
$21^{g}$ & C7 & DCM & 24 & 50 & 23.8 \\
$22^{g}$ & C7 & DCE & 24 & 36 & 9.4 \\
$23^{g}$ & C7 & CHCl & 24 & 58 & 20.0 \\
$24^{g}$ & C7 & toluene & 24 & 28 & 3.7 \\
$25^{g}$ & C7 & THF & 24 & 11 & 2.5 \\
$26^{g}$ & Quinidine & DCM & 24 & 27 & 7.4 \\
$27^{g}$ & Quinine & DCM & 24 & 33 & 11.3 \\
\hline
\end{tabular}

${ }^{a}$ The reaction was carried out with $\mathbf{1 a}(31.0 \mathrm{mg}, 0.10 \mathrm{mmol}), \mathrm{Ag}_{2} \mathrm{O}(27.8 \mathrm{mg}, 0.12 \mathrm{mmol})$ in DCM (2 $\mathrm{mL}$ ) for $2 \mathrm{~h}$ at $25^{\circ} \mathrm{C}$, the mixture was filtered and the filtrate was concentrated in vacuo to give $\mathbf{2 a}$, which was used directly. Then 2a, 2-naphthol 3a (27.8 mg, $0.12 \mathrm{mmol})$, CPA (5 mol \%) was added in solvent $(2 \mathrm{~mL})$ at r.t. ${ }^{b}$ Isolated yield based on 1a. ${ }^{c}$ ee values were determined by chiral HPLC (FLM Chiral ND (2) column). ${ }^{d} 0{ }^{\circ} \mathrm{C},{ }^{e}-30{ }^{\circ} \mathrm{C},{ }^{f} \mathrm{~N}_{2}$ protection, ${ }^{g} \mathrm{MgSO}_{4}$ was added (36.1 mg, 3.0 eq).

\section{2、Table S2-Optimization of the Reaction Conditions with quinone $2 \mathrm{~b}$ as the}

\section{model substrate ${ }^{a}$}
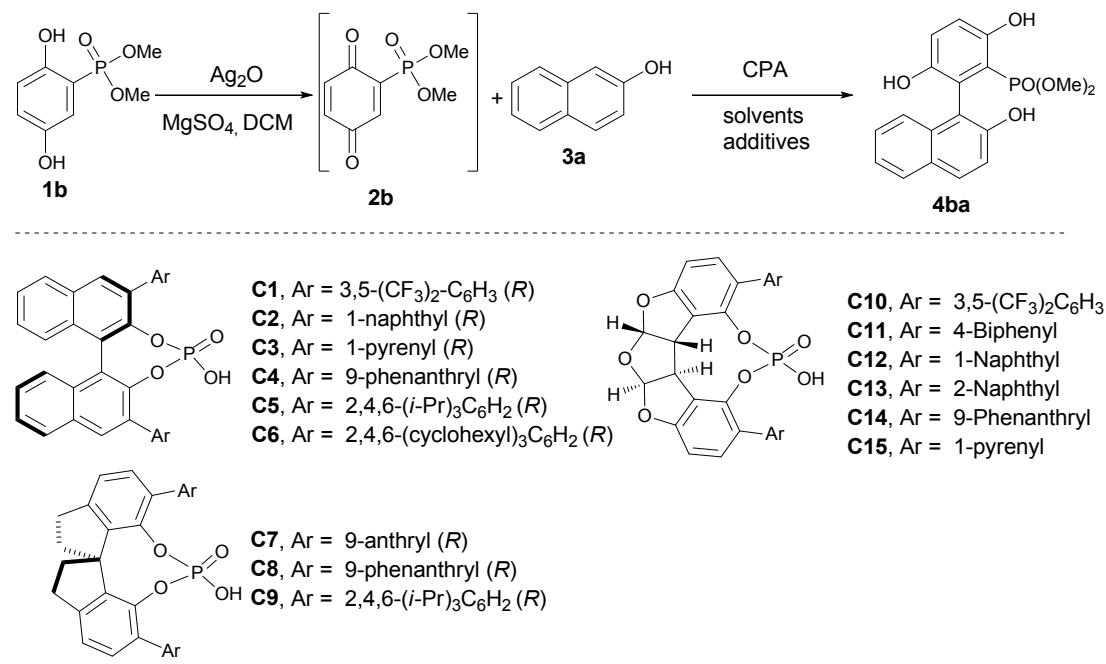

\begin{tabular}{cccccc}
\hline Entry & Catalyst & Solvent & Additives & Yield (\%) $^{\mathbf{b}}$ & ee (\%) \\
\hline $1^{\mathrm{d}}$ & $\mathrm{C} 7$ & $\mathrm{DCM}$ & $\mathrm{MgSO}_{4}$ & 60 & 69 \\
$2^{\mathrm{e}}$ & $\mathrm{C} 7$ & $\mathrm{DCM}$ & $\mathrm{MgSO}_{4}$ & 96 & 83 \\
3 & $\mathrm{C} 7$ & $\mathrm{DCM}$ & $\mathrm{MgSO}_{4}$ & $\mathbf{9 4}$ & $\mathbf{8 5}$
\end{tabular}




\begin{tabular}{|c|c|c|c|c|c|}
\hline 4 & $\mathrm{C} 1$ & DCM & $\mathrm{MgSO}_{4}$ & 56 & -14 \\
\hline 5 & $\mathrm{C} 2$ & DCM & $\mathrm{MgSO}_{4}$ & 76 & 29 \\
\hline 6 & $\mathrm{C} 3$ & DCM & $\mathrm{MgSO}_{4}$ & 58 & 1 \\
\hline 7 & $\mathrm{C} 4$ & DCM & $\mathrm{MgSO}_{4}$ & 77 & -20 \\
\hline 8 & $\mathrm{C} 5$ & DCM & $\mathrm{MgSO}_{4}$ & 85 & -78 \\
\hline 9 & C6 & DCM & $\mathrm{MgSO}_{4}$ & 75 & -72 \\
\hline 10 & $\mathrm{C} 8$ & DCM & $\mathrm{MgSO}_{4}$ & 86 & 43 \\
\hline 11 & C9 & DCM & $\mathrm{MgSO}_{4}$ & 43 & 40 \\
\hline $12^{\mathrm{f}}$ & $\mathrm{C} 10$ & DCM & $\mathrm{MgSO}_{4}$ & 66 & -11 \\
\hline $13^{f}$ & $\mathrm{C} 11$ & DCM & $\mathrm{MgSO}_{4}$ & 76 & -1 \\
\hline $14^{\mathrm{f}}$ & $\mathrm{C} 12$ & DCM & $\mathrm{MgSO}_{4}$ & 59 & -4 \\
\hline $15^{f}$ & $\mathrm{C} 13$ & DCM & $\mathrm{MgSO}_{4}$ & 69 & 5 \\
\hline $16^{\mathrm{f}}$ & $\mathrm{C} 14$ & DCM & $\mathrm{MgSO}_{4}$ & 67 & -17 \\
\hline $17^{\mathrm{f}}$ & $\mathrm{C} 15$ & DCM & $\mathrm{MgSO}_{4}$ & 66 & -8 \\
\hline 18 & $\mathrm{C} 7$ & DCM & $3 \AA \AA \mathrm{MS}$ & 39 & 11 \\
\hline 19 & C7 & DCM & $4 \AA ̊ \mathrm{MS}$ & 62 & 1 \\
\hline 20 & C7 & DCM & $5 \AA \mathrm{MS}$ & 61 & 4 \\
\hline $21^{\mathrm{g}}$ & C7 & DCM & - & 96 & 82 \\
\hline $22^{\mathrm{g}}$ & $\mathrm{C} 7$ & DCE & - & 91 & 86 \\
\hline $23^{g}$ & C7 & $\mathrm{CHCl}_{3}$ & - & 89 & 59 \\
\hline $24^{\mathrm{g}}$ & $\mathrm{C} 7$ & Toluene & - & 82 & 70 \\
\hline $25^{\mathrm{g}, \mathrm{h}}$ & C7 & DCE & - & 96 & 92 \\
\hline $26^{\mathrm{g}, \mathrm{i}}$ & $\mathrm{C} 7$ & DCM & - & 74 & 87 \\
\hline $27^{g . j}$ & C7 & DCE & - & 92 & 91 \\
\hline
\end{tabular}

${ }^{a}$ The reaction was carried out with $\mathbf{1 b}(0.10 \mathrm{mmol}), \mathrm{Ag}_{2} \mathrm{O}(27.8 \mathrm{mg}, 0.12 \mathrm{mmol})$ and $\mathrm{MgSO}_{4}(36.1$ $\mathrm{mg}, 0.30 \mathrm{mmol})$ in DCM $(2.0 \mathrm{~mL})$ for $2 \mathrm{~h}$ at $25{ }^{\circ} \mathrm{C}$, the mixture was filtered and the filtrate was concentrated in vacuo to give $\mathbf{2 b}$, which was used directly. Then $\mathbf{2 b}$, 2-naphthol 3a (17.3 mg, 0.12 $\mathrm{mmol})$, CPA (0.015 mmol, $5 \mathrm{~mol} \%)$ was added in solvent $(2 \mathrm{~mL})$ at $-20{ }^{\circ} \mathrm{C} ;{ }^{b}$ isolated yields; ${ }^{c}$ determined by chiral HPLC using a FLM Chiral column; ${ }^{d}$ the reaction was operated at r.t.; ${ }^{e}$ the reaction was operated at $0{ }^{\circ} \mathrm{C} ;{ }^{f}$ details for $\mathbf{C 1 0}-\mathbf{C 1 5}$ see ref $1 ;{ }^{g}$ no additive was used; $g$ the reaction was operated at $-35^{\circ} \mathrm{C} ;{ }^{i}$ the reaction was operated at $-78^{\circ} \mathrm{C} ;{ }^{j} 2.5 \mathrm{~mol} \%$ of $\mathbf{C} 7$ was used. 


\section{3、Table S3-Substrates scope of quinones with different phosphonate esters ${ }^{\mathrm{a}}$}

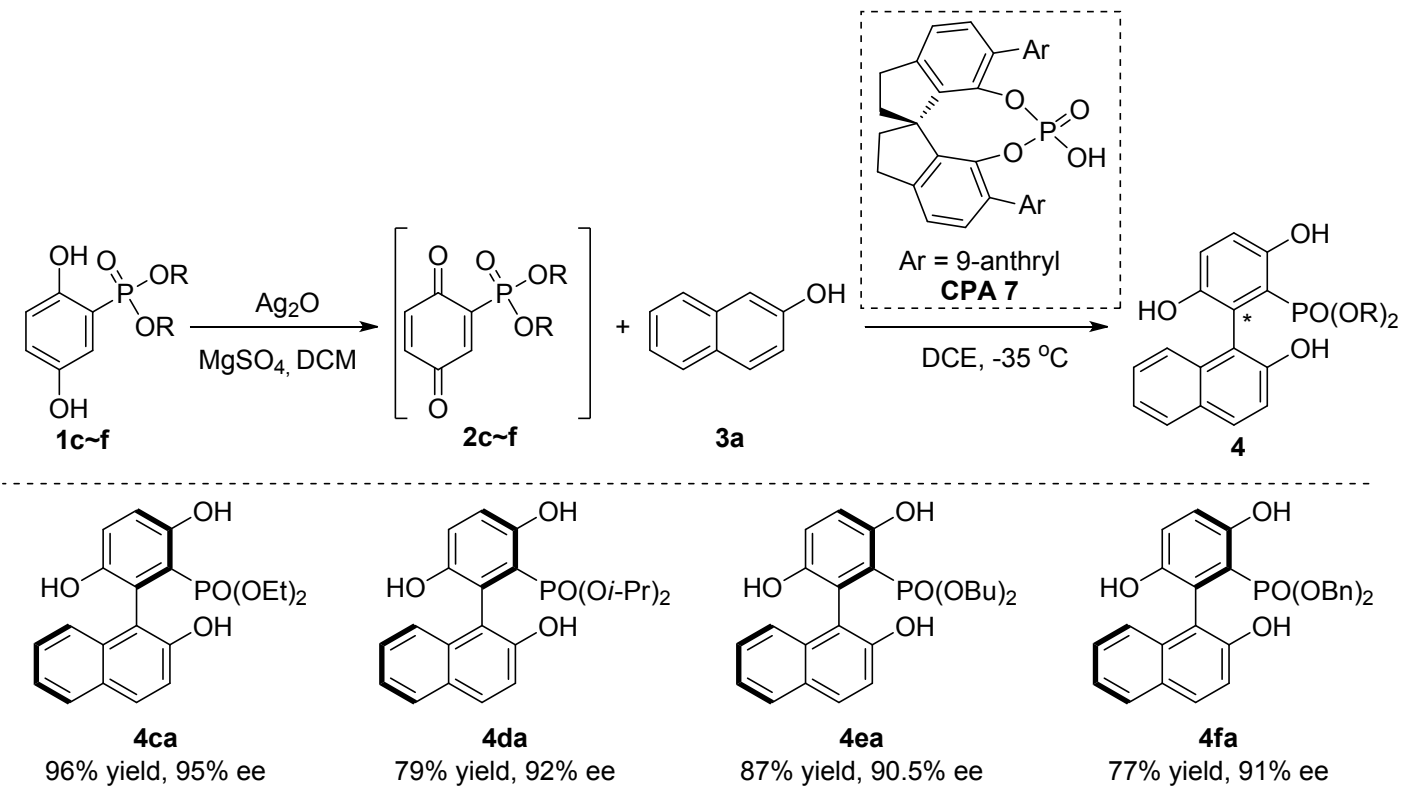

${ }^{\text {a }}$ The reaction was carried out with $\mathbf{1} \sim \mathbf{f}(0.30 \mathrm{mmol}), \mathrm{Ag}_{2} \mathrm{O}(83.4 \mathrm{mg}, 0.36 \mathrm{mmol})$ and $\mathrm{MgSO}_{4}$ (108.3mg, $0.90 \mathrm{mmol})$ in DCM $(6.0 \mathrm{~mL})$ for $2 \mathrm{~h}$ at $25^{\circ} \mathrm{C}$, the mixture was filtered and the filtrate was concentrated in vacuo to give $\mathbf{2 c} \sim \mathbf{f}$, which was used directly. Then $\mathbf{2 c} \sim \mathbf{f}$, 2-naphthol 3a (51.9 $\mathrm{mg}, 0.36 \mathrm{mmol})$, and $\mathbf{C} 7\left(5.0 \mathrm{mg}, 0.0075 \mathrm{mmol}, 2.5 \mathrm{~mol} \%\right.$ ) was added in DCE (6 mL) at $-35^{\circ} \mathrm{C}$.

\section{4、Table S4-Substrates scope of 2-naphthols and phenols ${ }^{\mathrm{a}}$}

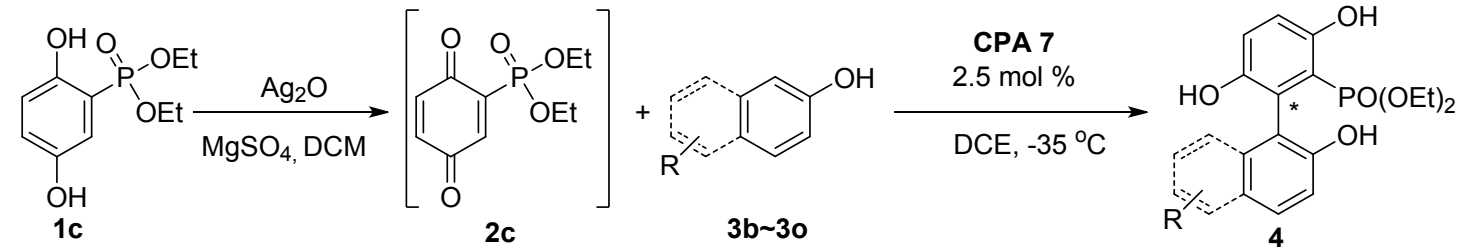

\begin{tabular}{ccccccc}
\hline Entry & $\mathbf{3}$ & Time(h) & $\mathbf{4}$ & $\mathrm{W}(\mathrm{mg})$ & Yield (\%) & ee (\%) \\
\hline 1 & 3-Br-2-naphthol & 48 & $\mathbf{4 c b}$ & 25.0 & 19 & 57 \\
2 & 6-Br-2-naphthol & 27 & $\mathbf{4 c c}$ & 32.4 & 23 & 88 \\
3 & 7-Br-2-naphthol & 29 & $\mathbf{4 c d}$ & 25.3 & 29 & 88 \\
4 & 6-Me-2-naphthol & 24 & $\mathbf{4 c m}$ & 119.0 & 99 & 95.3 \\
5 & 6-OMe-2-naphthol & 24 & $\mathbf{4 c e}$ & 60.4 & 48 & 89.1 \\
6 & 7-OMe-2-naphthol & 24 & $\mathbf{4 c f}$ & 106.4 & 85 & 93.1 \\
7 & 6-Ph-2-naphthol & 27 & $\mathbf{4 c g}$ & 94.2 & 68 & 92.1 \\
8 & 7-Ph-2-naphthol & 24 & $\mathbf{4 c h}$ & 118.2 & 85 & 93.5 \\
\hline
\end{tabular}




\begin{tabular}{ccccccc}
\hline 9 & 7-OEt-2-naphthol & 24 & $\mathbf{4 c i}$ & 127.3 & 98 & 92.2 \\
10 & 7-OPr-2-naphthol & 26 & $\mathbf{4 c j}$ & 139.5 & $>99$ & 93.9 \\
11 & 7-OBu-2-naphthol & 24 & $\mathbf{4 c k}$ & 137.0 & 99 & 91.3 \\
12 & 7-OBn-2-naphthol & 24 & $\mathbf{4 c l}$ & 143.5 & 97 & 94.6 \\
13 & 3,5-dimethoxyphenol & 24 & $\mathbf{4 c n}$ & 90.2 & 76 & 74 \\
14 & 4H-2-naphthol & 48 & $\mathbf{4 c o}$ & $\mathrm{NR}^{\mathrm{b}}$ & - & - \\
\hline
\end{tabular}

a The reaction was carried out with $1 \mathrm{c}(0.30 \mathrm{mmol}), \mathrm{Ag}_{2} \mathrm{O}(83.4 \mathrm{mg}, 0.36 \mathrm{mmol})$ and $\mathrm{MgSO}_{4}$ (108.3mg, $0.90 \mathrm{mmol})$ in DCM $(6.0 \mathrm{~mL})$ for $2 \mathrm{~h}$ at $25^{\circ} \mathrm{C}$, the mixture was filtered and the filtrate was concentrated in vacuo to give $\mathbf{2 c}$, which was used directly. Then $\mathbf{2 c}, \mathbf{3 b} \sim \mathbf{3 o}(0.36 \mathrm{mmol}), \mathbf{C} 7$ $(5.0 \mathrm{mg}, 0.0075 \mathrm{mmol}, 2.5 \mathrm{~mol} \%)$ was added in DCE $(6 \mathrm{~mL})$ at $-35^{\circ} \mathrm{C} .{ }^{\mathrm{b}}$ no reaction.

\section{5、General procedure for asymmetric synthesis of 4aa}

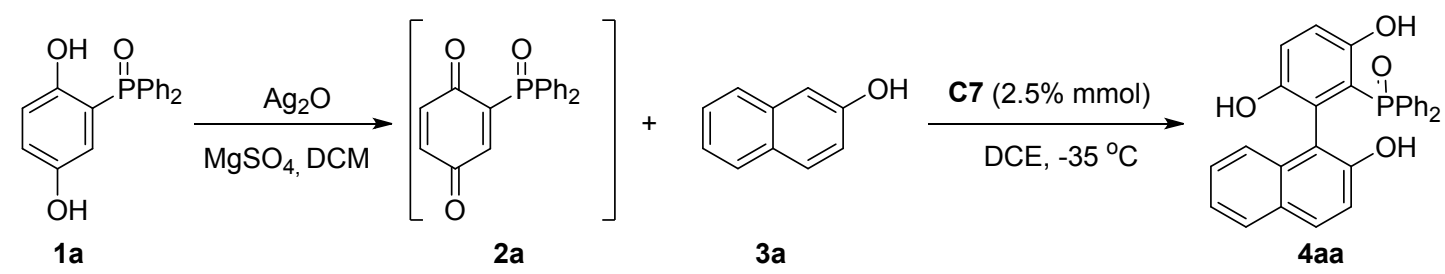

(3,6-Dihydroxy-2-(2-hydroxynaphthalen-1-yl)phenyl)diphenylphosphine oxide (4aa): To a mixture of $1 \mathrm{a}$ (105.9 mg, $0.34 \mathrm{mmol}, 1.0$ equiv), $\mathrm{Ag}_{2} \mathrm{O}$ (94.9 mg, $0.41 \mathrm{mmol}, 1.2$ equiv) and $\mathrm{MgSO}_{4}\left(123.2 \mathrm{mg}, 1.02 \mathrm{mmol}, 3.0\right.$ equiv) in $\mathrm{DCM}(7.0 \mathrm{~mL})$ for $2 \mathrm{~h}$ at $25{ }^{\circ} \mathrm{C}$, the mixture was filtered and the filtrate was concentrated in vacuo to give quinone 2a, which was used directly. Then 2a, 3a (59.1 mg, 0.0.41 mmol, 1.2 equiv), C7 (5.0 mg, $0.0075 \mathrm{mmol}, 2.5 \mathrm{~mol} \%$ ) was added in DCM $(7 \mathrm{~mL})$ at $-35^{\circ} \mathrm{C}$. This reaction mixture was stirred for $26 \mathrm{~h}$ at $-35^{\circ} \mathrm{C}$. The mixture was concentrated to remove the solvents. The residue was purified by column chromatography (PE/EA $=3 / 1)$ to give $4 \mathbf{a a}(38.3 \mathrm{mg}, 25 \%$ yield, $18 \%$ ee) as a white soild. $\mathrm{Rf}=0.34(\mathrm{PE} / \mathrm{EA}=1 / 1)$, m.p. $246.2-248.3{ }^{\circ} \mathrm{C} ;[\alpha]_{D}^{20}=+17.4(\mathrm{c}=0.5, \mathrm{EA}) ;{ }^{1} \mathrm{H}$ NMR $\left(400 \mathrm{MHz}, \mathrm{DMSO}-\mathrm{d}_{6}\right): \delta 10.71(\mathrm{~s}, 1 \mathrm{H})$, $9.17(\mathrm{~s}, 1 \mathrm{H}), 8.45(\mathrm{~s}, 1 \mathrm{H}), 7.50-7.34(\mathrm{~m}, 7 \mathrm{H}), 7.11-6.99(\mathrm{~m}, 6 \mathrm{H}), 6.95-6.74(\mathrm{~m}, 5 \mathrm{H}) ;{ }^{31} \mathrm{P}$ NMR $\left(162 \mathrm{MHz}, \mathrm{CDCl}_{3}\right) \delta: 36.5(\mathrm{~s}) ;{ }^{13} \mathrm{C}\left\{{ }^{1} \mathrm{H}\right\} \mathrm{NMR}\left(151 \mathrm{MHz}, \mathrm{CDCl}_{3}\right): \delta 155.4,155.3,152.5,148.34$, 148.26, 134.0, 133.7, 133.2, 132.9, 132.5, 132.3, 132.2, 132.0, 131.6, 131.5, 131.4, 131.0, 130.6, $130.52,130.50,129.3,128.8,128.5,127.74,127.65,127.5,127.3,126.8,126.7,126.31,126.25$, 125.2, 124.2, 121.7, 121.4, 117.7, 117.3, 117.2, 115.9, 115.8, 115.1, 114.4; HRMS (ESITOF) m/z: $[\mathrm{M}+\mathrm{H}]^{+}$calcd for $\left(\mathrm{C}_{28} \mathrm{H}_{22} \mathrm{O}_{4} \mathrm{P}\right), 453.1250$; found, 453.1228. Enantiometric excess: $18 \%$, determined by HPLC (FLM Chiral NY (2) column, hexane/EtOH 30:70, flow rate $0.5 \mathrm{~mL} / \mathrm{min}$, $\mathrm{t}_{\text {major }}=7.64 \mathrm{~min}, \mathrm{t}_{\text {minor }}=12.75 \mathrm{~min}, \lambda=210 \mathrm{~nm}$ ). 


\section{6、General procedure for asymmetric synthesis of chiral biaryl phosphonates}

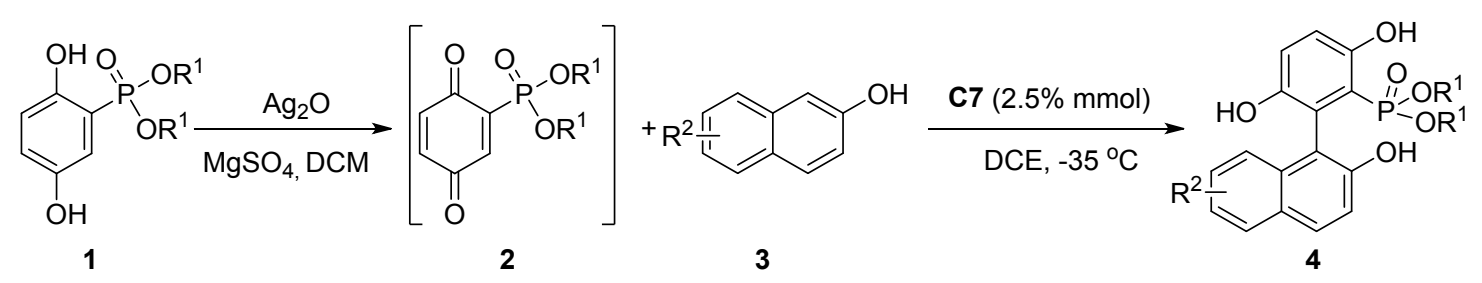

To a mixture of $1(0.30 \mathrm{mmol}), \mathrm{Ag}_{2} \mathrm{O}(83.4 \mathrm{mg}, 0.36 \mathrm{mmol})$ and $\mathrm{MgSO}_{4}(108.3 \mathrm{mg}, 0.90 \mathrm{mmol})$ in DCM $(6.0 \mathrm{~mL})$ for $2 \mathrm{~h}$ at $25^{\circ} \mathrm{C}$, the mixture was filtered and the filtrate was concentrated in vacuo to give 2, which was used directly. Then $\mathbf{2 , 3}(0.36 \mathrm{mmol}), \mathbf{C} 7(5.0 \mathrm{mg}, 0.0075 \mathrm{mmol}, 2.5 \mathrm{~mol} \%)$ was added in DCE $(6 \mathrm{~mL})$ at $-35{ }^{\circ} \mathrm{C}$. This reaction mixture was monitored by TLC. Direct purification reaction mixture by column chromatography on a silica gel gave the desired products. The enantiomeric excess was determined by HPLC.

Dimethyl (3,6-dihydroxy-2-(2-hydroxynaphthalen-1-yl)phenyl)phosphonate (4ba). The product was isolated by flash chromatography (PE/EA $=3: 1$ ) as a white soild $(103.3 \mathrm{mg}, 96 \%)$, Rf $=0.42(\mathrm{PE} / \mathrm{EA}=1: 1)$, m.p. $219.1-221.2^{\circ} \mathrm{C} ;[\alpha]_{D}^{20}=+10.88\left(\mathrm{c}=0.5, \mathrm{CH}_{2} \mathrm{Cl}_{2}\right) ;{ }^{1} \mathrm{H}$ NMR $(400 \mathrm{MHz}$, $\left.\mathrm{CDCl}_{3}\right): \delta 10.72(\mathrm{~s}, 1 \mathrm{H}), 7.87(\mathrm{~d}, \mathrm{~J}=8.9 \mathrm{~Hz}, 1 \mathrm{H}), 7.83-7.81(\mathrm{~m}, 1 \mathrm{H}), 7.43-7.36(\mathrm{~m}, 2 \mathrm{H}), 7.30-7.23$ $(\mathrm{m}, 2 \mathrm{H}), 7.16-7.13(\mathrm{~m}, 1 \mathrm{H}), 7.12-7.06(\mathrm{~m}, 1 \mathrm{H}), 5.25(\mathrm{~s}, 1 \mathrm{H}), 4.52(\mathrm{~s}, 1 \mathrm{H}), 3.50(\mathrm{~d}, \mathrm{~J}=11.0 \mathrm{~Hz}$, $3 \mathrm{H}), 2.97(\mathrm{~d}, \mathrm{~J}=11.4 \mathrm{~Hz}, 3 \mathrm{H}) ;{ }^{31} \mathrm{P}$ NMR $\left(162 \mathrm{MHz}, \mathrm{CDCl}_{3}\right) \delta: 22.9(\mathrm{~s}) ;{ }^{13} \mathrm{C}\left\{{ }^{1} \mathrm{H}\right\}$ NMR $(101$ $\left.\mathrm{MHz}, \mathrm{CDCl}_{3}\right): \delta 158.3,158.2,152.5,147.6,147.4,133.6,131.5,128.9,128.2,127.0,124.0$, 123.9, 123.8, 120.8, 120.7, 119.9, 117.8, 112.02, 111.97, 109.1, 107.3, 53.12, 53.06, 52.98, 52.92; HRMS (ESITOF) m/z: $[\mathrm{M}+\mathrm{H}]^{+}$calcd for $\left(\mathrm{C}_{18} \mathrm{H}_{18} \mathrm{O}_{6} \mathrm{P}\right)$, 361.0836; found, 361.0823. Enantiometric excess: 92\%, determined by HPLC (FLM Chiral NQ (2) column, hexane/EtOH 65:35, flow rate $0.5 \mathrm{~mL} / \mathrm{min}, \mathrm{t}_{\text {major }}=11.17 \mathrm{~min}, \mathrm{t}_{\text {minor }}=14.71 \mathrm{~min}, \lambda=210 \mathrm{~nm}$ ).

Diethyl (3,6-dihydroxy-2-(2-hydroxynaphthalen-1-yl)phenyl)phosphonate (4ca). The product was isolated by flash chromatography $(\mathrm{PE} / \mathrm{EA}=3: 1)$ as a white soild $(112.1 \mathrm{mg}, 96 \%), \mathrm{Rf}=0.31$ $(\mathrm{PE} / \mathrm{EA}=3: 1)$, m.p. $182.8-184.6{ }^{\circ} \mathrm{C} ;[\alpha]_{D}^{20}=+11.9\left(\mathrm{c}=0.5, \mathrm{CH}_{2} \mathrm{Cl}_{2}\right) ;{ }^{1} \mathrm{H} \mathrm{NMR}\left(400 \mathrm{MHz}, \mathrm{CDCl}_{3}\right)$ : $\delta 10.86(\mathrm{~s}, 1 \mathrm{H}), 7.89(\mathrm{~d}, J=8.9 \mathrm{~Hz}, 1 \mathrm{H}), 7.84(\mathrm{dd}, J=6.6,2.7 \mathrm{~Hz}, 1 \mathrm{H}), 7.42-7.32(\mathrm{~m}, 2 \mathrm{H})$, 7.28-7.26 (m, 2H), 7.21-7.16 (m, 1H), 7.10 (dd, $J=8.9,7.3 \mathrm{~Hz}, 1 \mathrm{H}), 5.24(\mathrm{~s}, 1 \mathrm{H}), 4.51(\mathrm{~s}, 1 \mathrm{H})$, 3.98-3.81 (m, 2H), 3.54-3.37 (m, 2H), $1.22(\mathrm{t}, J=7.1 \mathrm{~Hz}, 3 \mathrm{H}), 0.46(\mathrm{t}, J=7.0 \mathrm{~Hz}, 3 \mathrm{H}) ;{ }^{31} \mathrm{P}$ NMR $\left(162 \mathrm{MHz}, \mathrm{CDCl}_{3}\right) \delta: 20.5(\mathrm{~s}) ;{ }^{13} \mathrm{C}\left\{{ }^{1} \mathrm{H}\right\} \operatorname{NMR}\left(101 \mathrm{MHz}, \mathrm{CDCl}_{3}\right): \delta 157.93,157.86,152.5,147.5$, 147.3, 133.7, 131.4, 129.0, 128.1, 127.1, 124.2, 123.8, 123.7, 123.6, 120.8, 120.7, 119.8, 119.7, $117.9,112.34,112.30,100.2,108.4,63.0,62.9,62.72,62.66,15.83,15.77,14.9,14.8$; HRMS (ESITOF) $\mathrm{m} / \mathrm{z}:[\mathrm{M}+\mathrm{H}]^{+}$calcd for $\left(\mathrm{C}_{20} \mathrm{H}_{22} \mathrm{O}_{6} \mathrm{P}\right), 389.1149$; found, 389.1149. Enantiometric excess: 95\%, determined by HPLC (FLM Chiral ND (2) column, hexane/EtOH 50:50, flow rate $\left.0.5 \mathrm{~mL} / \mathrm{min}, \mathrm{t}_{\text {major }}=8.91 \mathrm{~min}, \mathrm{t}_{\text {minor }}=16.05 \mathrm{~min}, \lambda=210 \mathrm{~nm}\right)$.

Diisopropyl (3,6-dihydroxy-2-(2-hydroxynaphthalen-1-yl)phenyl)phosphonate (4da). The product was isolated by flash chromatography $(\mathrm{PE} / \mathrm{EA}=3: 1)$ as a white soild $(98.1 \mathrm{mg}, 79 \%), \mathrm{Rf}$ $=0.32(\mathrm{PE} / \mathrm{EA}=3: 1)$, m.p. $190.0-191.0{ }^{\circ} \mathrm{C} ;[\alpha]_{D}^{20}=+15.7\left(\mathrm{c}=0.5, \mathrm{CH}_{2} \mathrm{Cl}_{2}\right) ;{ }^{1} \mathrm{H}$ NMR $(400 \mathrm{MHz}$, $\mathrm{CDCl}_{3}$ ): $\delta 10.89(\mathrm{~s}, 1 \mathrm{H}), 7.86(\mathrm{dd}, J=8.8,2.9 \mathrm{~Hz}, 1 \mathrm{H}), 7.80(\mathrm{~d}, J=6.0 \mathrm{~Hz}, 1 \mathrm{H}), 7.35(\mathrm{dd}, J=3.5$, $2.3 \mathrm{~Hz}, 2 \mathrm{H}), 7.30-7.15(\mathrm{~m}, 3 \mathrm{H}), 7.12-7.01(\mathrm{~m}, 1 \mathrm{H}), 5.13(\mathrm{~d}, J=12.0 \mathrm{~Hz}, 1 \mathrm{H}), 4.55-4.54(\mathrm{~m}, 1 \mathrm{H})$, $4.41(\mathrm{~d}, J=12.4 \mathrm{~Hz}, 1 \mathrm{H}), 4.30-4.25(\mathrm{~m}, 1 \mathrm{H}), 1.27(\mathrm{~d}, J=5.8 \mathrm{~Hz}, 3 \mathrm{H}), 1.05(\mathrm{~d}, J=6.1 \mathrm{~Hz}, 3 \mathrm{H})$, 
$0.78(\mathrm{~d}, J=6.1 \mathrm{~Hz}, 3 \mathrm{H}), 0.35(\mathrm{~d}, J=5.6 \mathrm{~Hz}, 3 \mathrm{H}) ;{ }^{31} \mathrm{P} \mathrm{NMR}\left(162 \mathrm{MHz}, \mathrm{CDCl}_{3}\right) \delta: 18.7(\mathrm{~s}) ;$ ${ }^{13} \mathrm{C}\left\{{ }^{1} \mathrm{H}\right\}$ NMR $\left(101 \mathrm{MHz}, \mathrm{CDCl}_{3}\right): \delta 156.8,156.7,152.4,147.4,147.3,133.8,131.2,129.1,127.9$, 127.0, 124.9, 123.8, 123.2, 123.1, 120.9, 120.8, 119.5, 119.4, 118.1, 112.8, 112.77, 112.4, 110.5, 72.14, 72.1, 71.8, 71.7, 23.8, 23.7, 23.22, 23.19, 23.17, 23.15, 22.3, 22.2; HRMS (ESITOF) m/z: $[\mathrm{M}+\mathrm{H}]^{+}$calcd for $\left(\mathrm{C}_{22} \mathrm{H}_{26} \mathrm{O}_{6} \mathrm{P}\right), 417.1462$; found, 417.1450. Enantiometric excess: $92 \%$, determined by HPLC (FLM Chiral ND (2) column, hexane/EtOH 50:50, flow rate $0.5 \mathrm{~mL} / \mathrm{min}$, $\left.\mathrm{t}_{\text {major }}=7.94 \mathrm{~min}, \mathrm{t}_{\text {minor }}=16.25 \mathrm{~min}, \lambda=210 \mathrm{~nm}\right)$.

Dibutyl (3,6-dihydroxy-2-(2-hydroxynaphthalen-1-yl)phenyl)phosphonate (4ea). The product was isolated by flash chromatography $(\mathrm{PE} / \mathrm{EA}=3: 1)$ as a white soild $(116.3 \mathrm{mg}, 87 \%), \mathrm{Rf}=0.29$ $(\mathrm{PE} / \mathrm{EA}=3: 1)$, m.p. $133.1-135.2^{\circ} \mathrm{C} ;[\alpha]_{D}^{20}=+21.0\left(\mathrm{c}=0.5, \mathrm{CH}_{2} \mathrm{Cl}_{2}\right) ;{ }^{1} \mathrm{H} \mathrm{NMR}\left(400 \mathrm{MHz}, \mathrm{CDCl}_{3}\right)$ : $\delta 10.86(\mathrm{~s}, 1 \mathrm{H}), 7.86(\mathrm{~d}, J=8.9 \mathrm{~Hz}, 1 \mathrm{H}), 7.81(\mathrm{dd}, J=6.1,3.3 \mathrm{~Hz}, 1 \mathrm{H}), 7.34$ (dq, $J=6.6,3.4 \mathrm{~Hz}$, 2H), $7.24(\mathrm{~d}, J=9.0 \mathrm{~Hz}, 2 \mathrm{H}), 7.15(\mathrm{dd}, J=6.1,3.4 \mathrm{~Hz}, 1 \mathrm{H}), 7.08$ (dd, $J=9.0,7.3 \mathrm{~Hz}, 1 \mathrm{H}), 5.19$ (s, 1H), $4.47(\mathrm{~s}, 1 \mathrm{H}), 3.98-3.66(\mathrm{~m}, 2 \mathrm{H}), 3.52-3.24(\mathrm{~m}, 2 \mathrm{H}), 1.58-1.47(\mathrm{~m}, 2 \mathrm{H}), 1.25$ (dd, $J=15.0$, $7.5 \mathrm{~Hz}, 2 \mathrm{H}), 0.87$ (t, $J=7.4 \mathrm{~Hz}, 3 \mathrm{H}), 0.81-0.63(\mathrm{~m}, 4 \mathrm{H}), 0.60(\mathrm{t}, J=7.0 \mathrm{~Hz}, 3 \mathrm{H}) ;{ }^{31} \mathrm{P}$ NMR $(162$ $\left.\mathrm{MHz}, \mathrm{CDCl}_{3}\right) \delta: 20.5(\mathrm{~s}) ;{ }^{13} \mathrm{C}\left\{{ }^{1} \mathrm{H}\right\}$ NMR $\left(101 \mathrm{MHz}, \mathrm{CDCl}_{3}\right): \delta 157.9,157.8,152.5,147.5,147.3$, 133.7, 131.3, 129.0, 128.1, 127.1, 124.1, 123.6, 123.5, 120.8, 120.7, 119.8, 119.7, 117.9, 112.4, $112.3,110.3,108.5,66.7,66.6,66.5,66.4,32.1,32.0,31.22,31.15,18.5,18.1,13.5,13.3,22.2$; HRMS (ESITOF) m/z: $[\mathrm{M}+\mathrm{H}]^{+}$calcd for $\left(\mathrm{C}_{24} \mathrm{H}_{30} \mathrm{O}_{6} \mathrm{P}\right)$, 445.1775; found, 445.1774. Enantiometric excess: 91\%, determined by HPLC (FLM Chiral ND (2) column, hexane/EtOH 50:50, flow rate $0.5 \mathrm{~mL} / \mathrm{min}, \mathrm{t}_{\text {major }}=8.07 \mathrm{~min}, \mathrm{t}_{\text {minor }}=17.78 \mathrm{~min}, \lambda=210 \mathrm{~nm}$ ).

Dibenzyl (3,6-dihydroxy-2-(2-hydroxynaphthalen-1-yl)phenyl)phosphonate (4fa). The product was isolated by flash chromatography (PE/EA $=3: 1)$ as a white soild $(118.9 \mathrm{mg}, 77 \%), \mathrm{Rf}$ $=0.28(\mathrm{PE} / \mathrm{EA}=3: 1)$, m.p. $176.2-178.3^{\circ} \mathrm{C} ;[\alpha]_{D}^{20}=+32.5\left(\mathrm{c}=0.5, \mathrm{CH}_{2} \mathrm{Cl}_{2}\right) ;{ }^{1} \mathrm{H} \mathrm{NMR}(400 \mathrm{MHz}$, $\left.\mathrm{CDCl}_{3}\right): \delta 10.82(\mathrm{~s}, 1 \mathrm{H}), 7.82(\mathrm{t}, J=9.5 \mathrm{~Hz}, 2 \mathrm{H}), 7.30-7.24(\mathrm{~m}, 6 \mathrm{H}), 7.22-7.07(\mathrm{~m}, 8 \mathrm{H}), 6.53(\mathrm{~d}, J$ $=7.3 \mathrm{~Hz}, 2 \mathrm{H}), 4.82(\mathrm{dd}, J=11.5,8.3 \mathrm{~Hz}, 1 \mathrm{H}), 4.69(\mathrm{dd}, J=11.4,8.8 \mathrm{~Hz}, 1 \mathrm{H}), 4.41(\mathrm{dd}, J=11.3$, $9.0 \mathrm{~Hz}, 1 \mathrm{H}), 4.29(\mathrm{dd}, J=11.4,8.2 \mathrm{~Hz}, 1 \mathrm{H}), 3.33(\mathrm{br}, 2 \mathrm{H}) ;{ }^{31} \mathrm{P}$ NMR $\left(162 \mathrm{MHz}, \mathrm{CDCl}_{3}\right) \delta: 21.1$ (s); ${ }^{13} \mathrm{C}\left\{{ }^{1} \mathrm{H}\right\}$ NMR $\left(101 \mathrm{MHz}, \mathrm{CDCl}_{3}\right): \delta 157.9,157.8,152.4,147.5,147.3,135.24,135.17,135.0$, 134.9, 133.6, 131.3, 128.5, 128.4, 128.3, 128.1, 128.08, 127.6, 127.3, 124.1, 123.9, 123.88, 120.9, 120.7, 120.0, 119.9, 118.0, 112.3, 112.2, 110.1, 108.3, 68.4, 68.3, 68.12, 68.06; HRMS (ESITOF) $\mathrm{m} / \mathrm{z}:[\mathrm{M}+\mathrm{H}]^{+}$calcd for $\left(\mathrm{C}_{30} \mathrm{H}_{26} \mathrm{O}_{6} \mathrm{P}\right), 513.1462$; found, 513.1465. Enantiometric excess: $91 \%$, determined by HPLC (FLM Chiral ND (2) column, hexane/EtOH 20:80, flow rate $0.5 \mathrm{~mL} / \mathrm{min}$, $\mathrm{t}_{\text {major }}=8.98 \mathrm{~min}, \mathrm{t}_{\text {minor }}=24.44 \mathrm{~min}, \lambda=210 \mathrm{~nm}$ ).

Diethyl (2-(3-bromo-2-hydroxynaphthalen-1-yl)-3,6-dihydroxyphenyl)phosphonate (4cb). The product was isolated by flash chromatography $(\mathrm{PE} / \mathrm{EA}=5: 1)$ as a white soild $(25.0 \mathrm{mg}, 19 \%)$, $\mathrm{Rf}=0.59(\mathrm{PE} / \mathrm{EA}=2: 1)$, m.p. $225.6-227.7{ }^{\circ} \mathrm{C} ;[\alpha]_{D}^{20}=-16.6\left(\mathrm{c}=0.5, \mathrm{CH}_{2} \mathrm{Cl}_{2}\right) ;{ }^{1} \mathrm{H}$ NMR $(400$ MHz, DMSO-d $\mathrm{d}_{6}$ : $\delta 10.85(\mathrm{~s}, 1 \mathrm{H}), 8.82(\mathrm{~d}, J=18.8 \mathrm{~Hz}, 2 \mathrm{H}), 8.17(\mathrm{~s}, 1 \mathrm{H}), 7.85-7.69(\mathrm{~m}, 1 \mathrm{H}), 7.27$ $(\mathrm{dd}, J=6.2,3.1 \mathrm{~Hz}, 2 \mathrm{H}), 7.17(\mathrm{~d}, J=9.0 \mathrm{~Hz}, 1 \mathrm{H}), 6.98-6.84(\mathrm{~m}, 2 \mathrm{H}), 3.96-3.69(\mathrm{~m}, 2 \mathrm{H})$, 3.33-3.12 (m, 2H), $1.13(\mathrm{t}, J=7.0 \mathrm{~Hz}, 3 \mathrm{H}), 0.31(\mathrm{t}, J=7.0 \mathrm{~Hz}, 3 \mathrm{H}) ;{ }^{31} \mathrm{P}$ NMR $(162 \mathrm{MHz}$, DMSO-d $\left.{ }_{6}\right) \delta: 21.7(\mathrm{~s}) ;{ }^{13} \mathrm{C}\left\{{ }^{1} \mathrm{H}\right\}$ NMR (101 MHz, DMSO-d $\left.{ }_{6}\right): \delta 155.8,155.7,148.9,148.7,148.5$, 133.4, 131.0, 128.3, 126.6, 125.7, 124.8, 123.3, 123.2, 123.1, 118.4, 118.25, 118.20, 113.0, 110.4, 108.6, 62.43, 62.38, 61.47, 61.41, 15.6, 15.5, 14.63, 14.55; HRMS (ESITOF) m/z: $[\mathrm{M}+\mathrm{H}]^{+}$calcd for $\left(\mathrm{C}_{20} \mathrm{H}_{21} \mathrm{BrO}_{6} \mathrm{P}\right), 467.0254$; found, 467.0255 . Enantiometric excess: $57 \%$, determined by HPLC (FLM Chiral ND (2) column, hexane $/ \mathrm{EtOH} 40: 60$, flow rate $0.5 \mathrm{~mL} / \mathrm{min}, \mathrm{t}_{\text {major }}=8.01 \mathrm{~min}$, $\mathrm{t}_{\text {minor }}=$ 
$11.74 \mathrm{~min}, \lambda=210 \mathrm{~nm})$.

Diethyl (2-(6-bromo-2-hydroxynaphthalen-1-yl)-3,6-dihydroxyphenyl)phosphonate (4cc). The product was isolated by flash chromatography ( $\mathrm{PE} / \mathrm{EA}=5: 1)$ as a white soild $(32.4 \mathrm{mg}, 23 \%)$, $\mathrm{Rf}=0.30(\mathrm{PE} / \mathrm{EA}=2: 1)$, m.p. $167.1-169.0{ }^{\circ} \mathrm{C} ;[\alpha]_{D}^{20}=+75.0\left(\mathrm{c}=0.5, \mathrm{CH}_{2} \mathrm{Cl}_{2}\right) ;{ }^{1} \mathrm{H}$ NMR $(400$ MHz, DMSO-d $\mathrm{d}_{6}$ : $\delta 10.88(\mathrm{~s}, 1 \mathrm{H}), 9.43(\mathrm{~s}, 1 \mathrm{H}), 8.64(\mathrm{~s}, 1 \mathrm{H}), 8.02(\mathrm{~s}, 1 \mathrm{H}), 7.73(\mathrm{~d}, J=8.9 \mathrm{~Hz}, 1 \mathrm{H})$, $7.35(\mathrm{~d}, J=9.0 \mathrm{~Hz}, 1 \mathrm{H}), 7.23(\mathrm{dd}, J=8.7,21 \mathrm{~Hz}, 1 \mathrm{H}), 7.17-7.08(\mathrm{~m}, 1 \mathrm{H}), 6.96(\mathrm{~d}, J=8.7 \mathrm{~Hz}$, $1 \mathrm{H}), 6.84(\mathrm{dd}, J=11.3,4.8 \mathrm{~Hz}, 1 \mathrm{H}), 3.97-3.72(\mathrm{~m}, 2 \mathrm{H}), 3.36-3.30(\mathrm{~m}, 1 \mathrm{H}), 3.32-3.10(\mathrm{~m}, 1 \mathrm{H})$, $1.11(\mathrm{t}, J=6.9 \mathrm{~Hz}, 3 \mathrm{H}), 0.36(\mathrm{t}, J=7.0 \mathrm{~Hz}, 3 \mathrm{H}) ;{ }^{31} \mathrm{P}$ NMR $(162 \mathrm{MHz}$, DMSO-d 6 ) $\delta: 22.2(\mathrm{~s}) ;$ ${ }^{13} \mathrm{C}\left\{{ }^{1} \mathrm{H}\right\}$ NMR (151 MHz, DMSO-d $\left.\mathrm{d}_{6}\right): \delta 155.7,155.6,148.2,148.1,133.1,129.0,128.7,128.1$, $127.8,127.1,124.9,124.8,123.0,119.2,117.5,117.4,116.30,116.27,114.6,109.9,108.7,62.44$, $62.40,61.5,61.4,15.71,15.66,14.7,14.6$; HRMS (ESITOF) $\mathrm{m} / \mathrm{z}:[\mathrm{M}+\mathrm{H}]^{+}$calcd for $\left(\mathrm{C}_{20} \mathrm{H}_{21} \mathrm{BrO}_{6} \mathrm{P}\right)$, 467.0254; found, 467.0240. Enantiometric excess: $88 \%$, determined by HPLC (FLM Chiral NY (2) column, hexane/EtOH 80:20, flow rate $0.5 \mathrm{~mL} / \mathrm{min}, \mathrm{t}_{\text {major }}=14.54 \mathrm{~min}, \mathrm{t}_{\text {minor }}$ $=13.35 \mathrm{~min}, \lambda=210 \mathrm{~nm})$.

Diethyl (2-(7-bromo-2-hydroxynaphthalen-1-yl)-3,6-dihydroxyphenyl)phosphonate (4cd). The product was isolated by flash chromatography (PE/EA $=5: 1)$ as a white soild $(41.0 \mathrm{mg}, 29 \%)$, $\mathrm{Rf}=0.30(\mathrm{PE} / \mathrm{EA}=2: 1)$, m.p. $126.1-127.3{ }^{\circ} \mathrm{C} ;[\alpha]_{D}^{20}=+61.3\left(\mathrm{c}=0.5, \mathrm{CH}_{2} \mathrm{Cl}_{2}\right) ;{ }^{1} \mathrm{H}$ NMR $(400$ MHz, DMSO-d $\mathrm{d}_{6}$ : $\delta 10.88(\mathrm{~s}, 1 \mathrm{H}), 9.54(\mathrm{~s}, 1 \mathrm{H}), 8.71(\mathrm{~s}, 1 \mathrm{H}), 7.75(\mathrm{t}, J=9.1 \mathrm{~Hz}, 2 \mathrm{H}), 7.32(\mathrm{dd}, J=$ 8.6, 1.3 Hz, 1H), 7.22 (d, $J=8.8, \mathrm{~Hz}, 1 \mathrm{H}), 7.14$ (d, $J=8.9, \mathrm{~Hz}, 1 \mathrm{H}), 7.09$ (s, $1 \mathrm{H}), 6.87$ (t, $J=8.1$ $\mathrm{Hz}, 1 \mathrm{H}), 3.89-3.72(\mathrm{~m}, 2 \mathrm{H}), 3.46-3.40(\mathrm{~m}, 1 \mathrm{H}), 3.27-3.13(\mathrm{~m}, 1 \mathrm{H}), 1.09$ (t, $J=7.0 \mathrm{~Hz}, 3 \mathrm{H}), 0.42$ $(\mathrm{t}, J=7.0 \mathrm{~Hz}, 3 \mathrm{H}) ;{ }^{31} \mathrm{P}$ NMR $\left(162 \mathrm{MHz}, \mathrm{DMSO}_{-}\right) \delta: 22.1(\mathrm{~s}) ;{ }^{13} \mathrm{C}\left\{{ }^{1} \mathrm{H}\right\}$ NMR $(101 \mathrm{MHz}$, DMSO-d $_{6}$ ): $\delta 155.64,155.57,154.0,148.2,148.0,135.7,129.8,126.3,125.9,124.63,124.57$, 123.11, 123.09, 119.1, 118.7, 117.7, 117.6, 115.4, 115.3, 110.2, 108.5, 62.5, 62.4, 61.4, 61.3, 15.65, 15.57, 14.65, 14.57; HRMS (ESITOF) $\mathrm{m} / \mathrm{z}$ : $[\mathrm{M}+\mathrm{H}]^{+}$calcd for $\left(\mathrm{C}_{20} \mathrm{H}_{21} \mathrm{BrO}_{6} \mathrm{P}\right), 467.0254$; found, 467.0254. Enantiometric excess: 88\%, determined by HPLC (FLM Chiral ND (2) column, hexane/EtOH 40:60, flow rate $0.5 \mathrm{~mL} / \mathrm{min}, \mathrm{t}_{\text {major }}=7.81 \mathrm{~min}, \mathrm{t}_{\text {minor }}=11.12 \mathrm{~min}, \lambda=210 \mathrm{~nm}$ ).

Diethyl (3,6-dihydroxy-2-(2-hydroxy-6-methoxynaphthalen-1-yl)phenyl)phosphonate (4ce). The product was isolated by flash chromatography ( $\mathrm{PE} / \mathrm{EA}=3: 1)$ as a white soild $(60.4 \mathrm{mg}, 48 \%)$, $\mathrm{Rf}=0.32(\mathrm{PE} / \mathrm{EA}=2: 1)$, m.p. $158.4-160.3{ }^{\circ} \mathrm{C} ;[\alpha]_{D}^{20}=+34.0\left(\mathrm{c}=0.5, \mathrm{CH}_{2} \mathrm{Cl}_{2}\right) ;{ }^{1} \mathrm{H}$ NMR $(400$ $\left.\mathrm{MHz}, \mathrm{CDCl}_{3}\right): \delta 10.83(\mathrm{~s}, 1 \mathrm{H}), 7.76(\mathrm{~d}, J=8.9 \mathrm{~Hz}, 1 \mathrm{H}), 7.23(\mathrm{dd}, J=8.9,3.8 \mathrm{~Hz}, 2 \mathrm{H}), 7.14(\mathrm{~d}, J=$ $1.5 \mathrm{~Hz}, 1 \mathrm{H}), 7.11-7.00(\mathrm{~m}, 3 \mathrm{H}), 5.00(\mathrm{~s}, 1 \mathrm{H}), 4.44(\mathrm{~s}, 1 \mathrm{H}), 4.04-3.75(\mathrm{~m}, 5 \mathrm{H}), 3.61-3.34(\mathrm{~m}, 2 \mathrm{H})$, $1.19(\mathrm{t}, J=7.0 \mathrm{~Hz}, 3 \mathrm{H}), 0.54(\mathrm{t}, J=7.0 \mathrm{~Hz}, 3 \mathrm{H}) ;{ }^{31} \mathrm{P} \mathrm{NMR}\left(162 \mathrm{MHz}, \mathrm{CDCl}_{3}\right) \delta: 20.5(\mathrm{~s}) ;{ }^{13} \mathrm{C}\left\{{ }^{1} \mathrm{H}\right\}$ NMR $\left(101 \mathrm{MHz}, \mathrm{CDCl}_{3}\right): \delta 157.9,157.8,156.3,150.8,147.4,147.2,130.02,129.96,128.9$, $125.7,123.63,123.61,120.8,120.6,120.0,119.9,119.4,118.4,112.71,112.66,110.1,108.3$, 106.6, 63.0, 62.9, 62.8, 62.7, 55.4, 15.84, 15.78, 15.1, 15.0; HRMS (ESITOF) $\mathrm{m} / \mathrm{z}:[\mathrm{M}+\mathrm{H}]^{+}$calcd for $\left(\mathrm{C}_{21} \mathrm{H}_{24} \mathrm{O}_{7} \mathrm{P}\right), 419.1254$; found, 419.1252. Enantiometric excess: $89 \%$, determined by HPLC (FLM Chiral NY (2) column, hexane $/ \mathrm{EtOH} 40: 60$, flow rate $0.5 \mathrm{~mL} / \mathrm{min}, \mathrm{t}_{\text {major }}=8.93 \mathrm{~min}$, $\mathrm{t}_{\text {minor }}=$ $8.07 \mathrm{~min}, \lambda=210 \mathrm{~nm})$.

Diethyl (3,6-dihydroxy-2-(2-hydroxy-7-methoxynaphthalen-1-yl)phenyl)phosphonate (4cf). The product was isolated by flash chromatography $(\mathrm{PE} / \mathrm{EA}=4: 1)$ as a white soild $(106.4 \mathrm{mg}$, $85 \%), \mathrm{Rf}=0.33(\mathrm{PE} / \mathrm{EA}=2: 1)$, m.p. $162.7-164.8^{\circ} \mathrm{C} ;[\alpha]_{D}^{20}=+63.8\left(\mathrm{c}=0.5, \mathrm{CH}_{2} \mathrm{Cl}_{2}\right) ;{ }^{1} \mathrm{H} \mathrm{NMR}$ $\left(400 \mathrm{MHz}, \mathrm{CDCl}_{3}\right): \delta 10.86(\mathrm{~s}, 1 \mathrm{H}), 7.78(\mathrm{~d}, J=8.8 \mathrm{~Hz}, 1 \mathrm{H}), 7.71(\mathrm{~d}, J=8.9 \mathrm{~Hz}, 1 \mathrm{H}), 7.30-7.19$ (m, 1H), $7.08(\mathrm{t}, J=8.4 \mathrm{~Hz}, 2 \mathrm{H}), 7.00(\mathrm{dd}, J=8.9,2.4 \mathrm{~Hz}, 1 \mathrm{H}), 6.43(\mathrm{~d}, J=2.1 \mathrm{~Hz}, 1 \mathrm{H}), 5.11(\mathrm{~s}$, 1H), 4.50 (s, 1H), 3.89 (dddd, $J=24.7,14.9,7.5,2.7 \mathrm{~Hz}, 2 \mathrm{H}), 3.69$ (s, 3H), 3.46 (dddd, $J=16.7$, 
14.1, 7.1, 2.7 Hz, 2H), $1.20(\mathrm{t}, J=7.1 \mathrm{~Hz}, 3 \mathrm{H}), 0.50(\mathrm{t}, J=7.0 \mathrm{~Hz}, 3 \mathrm{H}) ;{ }^{31} \mathrm{P}$ NMR $(162 \mathrm{MHz}$, $\left.\mathrm{CDCl}_{3}\right) \delta: 20.4(\mathrm{~s}) ;{ }^{13} \mathrm{C}\left\{{ }^{1} \mathrm{H}\right\}$ NMR $\left(101 \mathrm{MHz}, \mathrm{CDCl}_{3}\right): \delta 158.8,158.0,157.9,153.1,147.4,147.3$, $135.2,131.1,129.7,124.3,123.7,123.6,120.9,120.7,119.8,119.76,115.8,115.3,111.42$, $111.38,110.1,108.3,103.3,63.1,63.0,62.7,62.6,55.2,15.9,15.8,14.93,14.85$; HRMS (ESITOF) $\mathrm{m} / \mathrm{z}:[\mathrm{M}+\mathrm{H}]^{+}$calcd for $\left(\mathrm{C}_{21} \mathrm{H}_{24} \mathrm{O}_{7} \mathrm{P}\right)$, 419.1254; found, 419.1249. Enantiometric excess: 93\%, determined by HPLC (FLM Chiral ND (2) column, hexane/EtOH 40:60, flow rate $\left.0.5 \mathrm{~mL} / \mathrm{min}, \mathrm{t}_{\text {major }}=8.22 \mathrm{~min}, \mathrm{t}_{\text {minor }}=16.54 \mathrm{~min}, \lambda=210 \mathrm{~nm}\right)$.

Diethyl (3,6-dihydroxy-2-(2-hydroxy-6-phenylnaphthalen-1-yl)phenyl)phosphonate (4cg). The product was isolated by flash chromatography $(\mathrm{PE} / \mathrm{EA}=2: 1)$ as a white soild $(94.2 \mathrm{mg}, 68 \%)$, $\mathrm{Rf}=0.25(\mathrm{PE} / \mathrm{EA}=2: 1)$, m.p. $212.1-214.1{ }^{\circ} \mathrm{C} ;[\alpha]_{D}^{20}=+82.7\left(\mathrm{c}=0.5, \mathrm{CH}_{2} \mathrm{Cl}_{2}\right) ;{ }^{1} \mathrm{H}$ NMR $(400$ $\left.\mathrm{MHz}, \mathrm{CDCl}_{3}\right): \delta 10.86(\mathrm{~s}, 1 \mathrm{H}), 8.03(\mathrm{~s}, 1 \mathrm{H}), 7.94(\mathrm{~d}, J=8.9 \mathrm{~Hz}, 1 \mathrm{H}), 7.65(\mathrm{dd}, J=13.4,8.6 \mathrm{~Hz}$, $3 \mathrm{H}), 7.48(\mathrm{t}, J=7.6 \mathrm{~Hz}, 2 \mathrm{H}), 7.38(\mathrm{t}, J=7.3 \mathrm{~Hz}, 1 \mathrm{H}), 7.32-7.21(\mathrm{~m}, 3 \mathrm{H}), 7.11(\mathrm{t}, J=8.1 \mathrm{~Hz}, 1 \mathrm{H})$, 5.30-5.18 (m, 1H), $4.49(\mathrm{~s}, 1 \mathrm{H}), 3.98-3.82(\mathrm{~m}, 2 \mathrm{H}), 3.61-3.38(\mathrm{~m}, 2 \mathrm{H}), 1.21(\mathrm{t}, J=7.0 \mathrm{~Hz}, 3 \mathrm{H})$, $0.48(\mathrm{t}, J=7.0 \mathrm{~Hz}, 3 \mathrm{H}) ;{ }^{31} \mathrm{P}$ NMR $\left(162 \mathrm{MHz}, \mathrm{CDCl}_{3}\right) \delta: 20.5(\mathrm{~d}, J=2.4 \mathrm{~Hz}) ;{ }^{13} \mathrm{C}\left\{{ }^{1} \mathrm{H}\right\} \mathrm{NMR}(101$ $\left.\mathrm{MHz}, \mathrm{CDCl}_{3}\right): \delta 158.0,157.9,152.6,147.5,147.3,140.7,136.7,132.9,131.6,129.2,128.9$, $127.3,127.1,126.6,126.0,124.8,123.74,123.72,120.9,120.8,119.7,119.6,118.4,112.34$, 112.30, 110.2, 108.4, 63.02, 62.95, 62.8, 62.7, 55.2, 15.9, 15.8, 14.93, 14.86; HRMS (ESITOF) $\mathrm{m} / \mathrm{z}:[\mathrm{M}+\mathrm{H}]^{+}$calcd for $\left(\mathrm{C}_{26} \mathrm{H}_{26} \mathrm{O}_{6} \mathrm{P}\right), 465.1462$; found, 465.1454. Enantiometric excess: $92 \%$, determined by HPLC (FLM Chiral ND (2) column, hexane/EtOH 40:60, flow rate $0.5 \mathrm{~mL} / \mathrm{min}$, $\left.\mathrm{t}_{\text {major }}=12.38 \mathrm{~min}, \mathrm{t}_{\text {minor }}=8.04 \mathrm{~min}, \lambda=210 \mathrm{~nm}\right)$.

Diethyl (3,6-dihydroxy-2-(2-hydroxy-7-phenylnaphthalen-1-yl)phenyl)phosphonate (4ch). The product was isolated by flash chromatography $(\mathrm{PE} / \mathrm{EA}=2: 1)$ as a white soild $(118.2 \mathrm{mg}$, $85 \%), \mathrm{Rf}=0.25(\mathrm{PE} / \mathrm{EA}=2: 1)$, m.p. $191.1-193.2{ }^{\circ} \mathrm{C} ;[\alpha]_{D}^{20}=+104.4\left(\mathrm{c}=0.5, \mathrm{CH}_{2} \mathrm{Cl}_{2}\right) ;{ }^{1} \mathrm{H} \mathrm{NMR}$ (400 MHz, $\mathrm{CDCl}_{3}$ ): $\delta 10.87(\mathrm{~s}, 1 \mathrm{H}), 7.90(\mathrm{dd}, J=8.7,2.1 \mathrm{~Hz}, 2 \mathrm{H}), 7.61(\mathrm{dd}, J=8.4,1.5 \mathrm{~Hz}, 1 \mathrm{H})$, $7.50(\mathrm{~d}, J=7.2 \mathrm{~Hz}, 2 \mathrm{H}), 7.42(\mathrm{t}, J=7.5 \mathrm{~Hz}, 2 \mathrm{H}), 7.38-7.30(\mathrm{~m}, 2 \mathrm{H}), 7.26(\mathrm{dd}, J=5.6,3.1 \mathrm{~Hz}$, 2H), 7.15-7.05 (m, 1H), $5.18(\mathrm{br}, 1 \mathrm{H}), 4.51(\mathrm{br}, 1 \mathrm{H}), 4.08-3.73(\mathrm{~m}, 2 \mathrm{H}), 3.58-3.24(\mathrm{~m}, 2 \mathrm{H}), 1.21$ $(\mathrm{t}, J=7.1 \mathrm{~Hz}, 3 \mathrm{H}), 0.37(\mathrm{t}, J=7.0 \mathrm{~Hz}, 3 \mathrm{H}) ;{ }^{31} \mathrm{P} \mathrm{NMR}\left(162 \mathrm{MHz}, \mathrm{CDCl}_{3}\right) \delta: 20.5(\mathrm{~s}) ;{ }^{13} \mathrm{C}\left\{{ }^{1} \mathrm{H}\right\}$ NMR $\left(101 \mathrm{MHz}, \mathrm{CDCl}_{3}\right): \delta 158.0,157.9,152.9,147.5,147.4,140.9,139.8,134.0,131.1,128.9$, $128.7,128.1,127.5,127.3,123.80,123.77,123.6,122.1,121.0,119.6,119.5,117.9,112.6,112.5$, $110.1,108.3,63.1,63.0,62.63,62.58,15.9,15.8,14.74,14.66$; HRMS (ESITOF) $\mathrm{m} / \mathrm{z}:[\mathrm{M}+\mathrm{H}]^{+}$ calcd for $\left(\mathrm{C}_{26} \mathrm{H}_{26} \mathrm{O}_{6} \mathrm{P}\right), 465.1462$; found, 465.1462. Enantiometric excess: $94 \%$, determined by HPLC (FLM Chiral NY (2) column, hexane/EtOH 40:60, flow rate $0.5 \mathrm{~mL} / \mathrm{min}$, $\mathrm{t}_{\text {major }}=7.13 \mathrm{~min}$, $\left.\mathrm{t}_{\mathrm{minor}}=9.45 \mathrm{~min}, \lambda=210 \mathrm{~nm}\right)$.

Diethyl (2-(7-ethoxy-2-hydroxynaphthalen-1-yl)-3,6-dihydroxyphenyl)phosphonate (4ci). The product was isolated by flash chromatography (PE/EA $=2: 1$ ) as a white soild $(127.3 \mathrm{mg}, 98 \%), \mathrm{Rf}$ $=0.25(\mathrm{PE} / \mathrm{EA}=2: 1)$, m.p. $124.0-125.2{ }^{\circ} \mathrm{C} ;[\alpha]_{D}^{20}=+62.3\left(\mathrm{c}=0.5, \mathrm{CH}_{2} \mathrm{Cl}_{2}\right) ;{ }^{1} \mathrm{H}$ NMR $(400 \mathrm{MHz}$, $\left.\mathrm{CDCl}_{3}\right): \delta 10.88(\mathrm{~s}, 1 \mathrm{H}), 7.79(\mathrm{~d}, J=8.8 \mathrm{~Hz}, 1 \mathrm{H}), 7.71(\mathrm{~d}, J=8.9 \mathrm{~Hz}, 1 \mathrm{H}), 7.27(\mathrm{t}, J=4.5 \mathrm{~Hz}$, $1 \mathrm{H}), 7.15-7.06(\mathrm{~m}, 2 \mathrm{H}), 7.01$ (dd, $J=8.9,2.3 \mathrm{~Hz}, 1 \mathrm{H}), 6.43$ (d, $J=1.9 \mathrm{~Hz}, 1 \mathrm{H}), 5.07$ (br, 1H), 4.46 (br, 1H), 4.03-3.81 (m, 4H), 3.47 (tdd, $J=9.7,8.3,4.9,2 \mathrm{H}), 1.37$ (t, $J=7.0 \mathrm{~Hz}, 3 \mathrm{H}), 1.22$ (t, $J=7.1 \mathrm{~Hz}, 3 \mathrm{H}), 0.51(\mathrm{t}, J=7.0 \mathrm{~Hz}, 3 \mathrm{H}) ;{ }^{31} \mathrm{P} \mathrm{NMR}\left(162 \mathrm{MHz}, \mathrm{CDCl}_{3}\right) \delta: 20.4(\mathrm{~s}) ;{ }^{13} \mathrm{C}\left\{{ }^{1} \mathrm{H}\right\} \mathrm{NMR}$ $\left(101 \mathrm{MHz}, \mathrm{CDCl}_{3}\right): \delta 158.1,158.0,157.9,153.1,147.5,147.3,135.2,131.1,129.7,124.3,123.7$, 123.6, 120.9, 120.7, 119.84, 119.78, 116.0, 115.2, 111.33, 111.29, 110.2, 108.3, 104.2, 63.4, 63.1, 63.0, 62.72, 62.66, 15.9, 15.8, 14.93, 14.86, 14.6; HRMS (ESITOF) $\mathrm{m} / \mathrm{z}:[\mathrm{M}+\mathrm{H}]^{+}$calcd for $\left(\mathrm{C}_{22} \mathrm{H}_{22} \mathrm{O}_{7} \mathrm{P}\right), 433.1411$; found, 433.1405 . Enantiometric excess: $92 \%$, determined by HPLC (FLM 
Chiral NY (2) column, hexane/EtOH 50:50, flow rate $0.5 \mathrm{~mL} / \mathrm{min}, \mathrm{t}_{\text {major }}=7.14 \mathrm{~min}, \mathrm{t}_{\operatorname{minor}}=8.53$ $\min , \lambda=236 \mathrm{~nm})$.

Diethyl (3,6-dihydroxy-2-(2-hydroxy-7-propoxynaphthalen-1-yl)phenyl)phosphonate (4cj). The product was isolated by flash chromatography $(\mathrm{PE} / \mathrm{EA}=2: 1)$ as a white soild $(139.5 \mathrm{mg},>$ $99 \%), \mathrm{Rf}=0.28(\mathrm{PE} / \mathrm{EA}=2: 1)$, m.p. $139.0-140.2{ }^{\circ} \mathrm{C} ;[\alpha]_{D}^{20}=+62.2\left(\mathrm{c}=0.5, \mathrm{CH}_{2} \mathrm{Cl}_{2}\right) ;{ }^{1} \mathrm{H} \mathrm{NMR}$ $\left(400 \mathrm{MHz}, \mathrm{CDCl}_{3}\right): \delta 10.87(\mathrm{~s}, 1 \mathrm{H}), 7.77(\mathrm{~d}, J=8.7 \mathrm{~Hz}, 1 \mathrm{H}), 7.70(\mathrm{~d}, J=8.9 \mathrm{~Hz}, 1 \mathrm{H}), 7.29-7.23$ (m, 1H), $7.09(\mathrm{t}, J=7.3 \mathrm{~Hz}, 2 \mathrm{H}), 7.01(\mathrm{~d}, J=8.9 \mathrm{~Hz}, 1 \mathrm{H}), 6.42(\mathrm{~s}, 1 \mathrm{H}), 5.11(\mathrm{~d}, J=12.7 \mathrm{~Hz}, 1 \mathrm{H})$, $4.51(\mathrm{~d}, J=6.1 \mathrm{~Hz}, 1 \mathrm{H}), 4.01-3.72(\mathrm{~m}, 4 \mathrm{H}), 3.55-3.36(\mathrm{~m}, 2 \mathrm{H}), 1.74(\mathrm{dt}, J=14.0,7.0 \mathrm{~Hz}, 2 \mathrm{H})$, $1.20(\mathrm{t}, J=7.0 \mathrm{~Hz}, 3 \mathrm{H}), 0.98(\mathrm{t}, J=7.4 \mathrm{~Hz}, 3 \mathrm{H}), 0.50(\mathrm{t}, J=7.0 \mathrm{~Hz}, 3 \mathrm{H}) ;{ }^{31} \mathrm{P}$ NMR $(162 \mathrm{MHz}$, $\left.\mathrm{CDCl}_{3}\right) \delta: 20.4(\mathrm{~s}) ;{ }^{13} \mathrm{C}\left\{{ }^{1} \mathrm{H}\right\}$ NMR (101 MHz, $\left.\mathrm{CDCl}_{3}\right): \delta 158.3,158.0,157.9,154.3,153.1,149.0$, $147.4,147.3,138.5,135.2,134.0,131.1,130.4,129.6,126.1,124.3,123.8,123.7,122.6,122.7$, $120.8,120.7,120.2,119.93,119.86,118.4,116.0,115.6,115.1,113.1,111.31,111.27,111.15$, 110.1, 110.0, 108.3, 107.6, 104.1, 69.4, 63.1, 63.0, 62.7, 62.6, 22.4, 15.9, 15.8, 14.9, 14.8, 10.5; HRMS (ESITOF) m/z: $[\mathrm{M}+\mathrm{H}]^{+}$calcd for $\left(\mathrm{C}_{23} \mathrm{H}_{28} \mathrm{O}_{7} \mathrm{P}\right)$, 447.1567; found, 447.1569. Enantiometric excess: 94\%, determined by HPLC (FLM Chiral NY (2) column, hexane/EtOH 50:50, flow rate $0.5 \mathrm{~mL} / \mathrm{min}, \mathrm{t}_{\text {major }}=7.13 \mathrm{~min}, \mathrm{t}_{\text {minor }}=8.57 \mathrm{~min}, \lambda=236 \mathrm{~nm}$ ).

Diethyl (2-(7-butoxy-2-hydroxynaphthalen-1-yl)-3,6-dihydroxyphenyl)phosphonate (4ck). The product was isolated by flash chromatography $(\mathrm{PE} / \mathrm{EA}=2: 1)$ as a white soild (137.0 $\mathrm{mg}$, 99\%), $\mathrm{Rf}=0.28(\mathrm{PE} / \mathrm{EA}=2: 1)$, m.p. $144.8-146.6{ }^{\circ} \mathrm{C} ;[\alpha]_{D}^{20}=+70.2\left(\mathrm{c}=0.5, \mathrm{CH}_{2} \mathrm{Cl}_{2}\right) ;{ }^{1} \mathrm{H}$ NMR $\left(400 \mathrm{MHz}, \mathrm{CDCl}_{3}\right): \delta 10.86(\mathrm{~s}, 1 \mathrm{H}), 7.76(\mathrm{~d}, J=8.8 \mathrm{~Hz}, 1 \mathrm{H}), 7.69$ (d, $\left.J=8.9 \mathrm{~Hz}, 1 \mathrm{H}\right), 7.25(\mathrm{dd}, J$ $=6.4,2.5 \mathrm{~Hz}, 1 \mathrm{H}), 7.08(\mathrm{dd}, J=11.7,5.9 \mathrm{~Hz}, 2 \mathrm{H}), 6.99(\mathrm{dd}, J=8.9,2.2 \mathrm{~Hz}, 1 \mathrm{H}), 6.41(\mathrm{~s}, 1 \mathrm{H})$, $5.11(\mathrm{~d}, J=15.6 \mathrm{~Hz}, 1 \mathrm{H}), 4.51(\mathrm{~d}, J=7.7 \mathrm{~Hz}, 1 \mathrm{H}), 3.99-3.75(\mathrm{~m}, 4 \mathrm{H}), 3.46$ (tdd, $J=16.6,9.7,7.1$, 2H), 1.75-1.65 (m, 2H), 1.49-1.36 (m, 2H), $1.19(\mathrm{t}, J=7.0 \mathrm{~Hz}, 3 \mathrm{H}), 0.92(\mathrm{t}, J=7.4 \mathrm{~Hz}, 3 \mathrm{H}), 0.49$ $(\mathrm{t}, J=7.0 \mathrm{~Hz}, 3 \mathrm{H}) ;{ }^{31} \mathrm{P}$ NMR $\left(162 \mathrm{MHz}, \mathrm{CDCl}_{3}\right) \delta: 20.5(\mathrm{~s}) ;{ }^{13} \mathrm{C}\left\{{ }^{1} \mathrm{H}\right\} \mathrm{NMR}\left(101 \mathrm{MHz}, \mathrm{CDCl}_{3}\right): \delta$ $158.3,158.0,157.9,153.1,147.5,147.3,135.2,131.1,129.6,124.2,123.64,123.62,120.8,120.7$, $119.94,119.88,116.0,115.1,111.31,111.27,110.1,110.0,108.3,104.1,70.4,67.5,66.6,63.1$, 63.0, 62.7, 62.6, 61.9, 31.1, 19.2, 15.9, 15.8, 14.9, 14.8, 13.8; HRMS (ESITOF) m/z: $[\mathrm{M}+\mathrm{H}]^{+}$ calcd for $\left(\mathrm{C}_{24} \mathrm{H}_{30} \mathrm{O}_{7} \mathrm{P}\right), 461.1724$; found, 461.1728. Enantiometric excess: $91 \%$, determined by HPLC (FLM Chiral NY (2) column, hexane/EtOH 50:50, flow rate $0.5 \mathrm{~mL} / \mathrm{min}$, $\mathrm{t}_{\text {major }}=7.01 \mathrm{~min}$, $\left.\mathrm{t}_{\text {minor }}=8.61 \mathrm{~min}, \lambda=210 \mathrm{~nm}\right)$.

Diethyl (2-(7-(benzyloxy)-2-hydroxynaphthalen-1-yl)-3,6-dihydroxyphenyl)phosphonate (4cl). The product was isolated by flash chromatography $(\mathrm{PE} / \mathrm{EA}=2: 1)$ as a white soild (143.5 $\mathrm{mg}, 97 \%), \mathrm{Rf}=0.25(\mathrm{PE} / \mathrm{EA}=2: 1)$, m.p. $167.6-169.7{ }^{\circ} \mathrm{C} ;[\alpha]_{D}^{20}=-3.0\left(\mathrm{c}=0.5, \mathrm{CH}_{2} \mathrm{Cl}_{2}\right) ;{ }^{1} \mathrm{H} \mathrm{NMR}$ $\left(400 \mathrm{MHz}, \mathrm{CDCl}_{3}\right): \delta 10.86(\mathrm{~s}, 1 \mathrm{H}), 7.75(\mathrm{~d}, J=8.8 \mathrm{~Hz}, 1 \mathrm{H}), 7.69(\mathrm{~d}, J=8.9 \mathrm{~Hz}, 1 \mathrm{H}), 7.36-7.25$ (m, 4H), 7.25-7.20 (m, 2H), 7.18-7.10 (m, 1H), 7.10-7.02 (m, 2H), $6.47(\mathrm{~d}, J=2.1 \mathrm{~Hz}, 1 \mathrm{H}), 5.16$ (s, 1H), $5.03(\mathrm{~d}, J=12.3 \mathrm{~Hz}, 1 \mathrm{H}), 4.86(\mathrm{~d}, J=12.3,1 \mathrm{H}), 4.50(\mathrm{~s}, 1 \mathrm{H}), 4.00-3.74(\mathrm{~m}, 2 \mathrm{H})$, 3.31-3.13 (m, 1H), 2.92-2.74 (m, 1H), $1.20(\mathrm{t}, J=7.0 \mathrm{~Hz}, 3 \mathrm{H}), 0.30(\mathrm{t}, J=7.0 \mathrm{~Hz}, 3 \mathrm{H}) ;{ }^{31} \mathrm{P}$ NMR $\left(162 \mathrm{MHz}, \mathrm{CDCl}_{3}\right) \delta: 20.4(\mathrm{~s}) ;{ }^{13} \mathrm{C}\left\{{ }^{1} \mathrm{H}\right\} \mathrm{NMR}\left(101 \mathrm{MHz}, \mathrm{CDCl}_{3}\right): \delta 158.1,158.0,157.8,153.0$, 147.4, 147.2, 136.8, 135.1, 131.0, 129.6, 128.6, 128.1, 127.6, 124.3, 123.63, 123.61, 120.7, 120.6, $120.0,119.9,116.5,115.4,111.5,111.4,110.1,108.3,104.7,70.0,62.9,62.8,62.5,62.4,15.83$, 15.76, 14.8, 14.7; HRMS (ESITOF) $\mathrm{m} / \mathrm{z}$ : $[\mathrm{M}+\mathrm{H}]^{+}$calcd for $\left(\mathrm{C}_{27} \mathrm{H}_{28} \mathrm{O}_{7} \mathrm{P}\right), 495.1567$; found, 461.1563. Enantiometric excess: 95\%, determined by HPLC (FLM Chiral NY (2) column, hexane/EtOH 50:50, flow rate $0.5 \mathrm{~mL} / \mathrm{min}, \mathrm{t}_{\text {major }}=8.39 \mathrm{~min}, \mathrm{t}_{\text {minor }}=10.99 \mathrm{~min}, \lambda=210 \mathrm{~nm}$ ).

Diethyl (3,6-dihydroxy-2-(2-hydroxy-6-methylnaphthalen-1-yl)phenyl)phosphonate $(4 \mathrm{~cm})$. 
The product was isolated by flash chromatography $(\mathrm{PE} / \mathrm{EA}=5: 1)$ as a white soild $(119.0 \mathrm{mg}$, 99\%), Rf $=0.32(\mathrm{PE} / \mathrm{EA}=2: 1)$, m.p. $151.0-153.1{ }^{\circ} \mathrm{C} ;[\alpha]_{D}^{20}=+31.6\left(\mathrm{c}=0.5, \mathrm{CH}_{2} \mathrm{Cl}_{2}\right) ;{ }^{1} \mathrm{H} \mathrm{NMR}$ $\left(400 \mathrm{MHz}, \mathrm{CDCl}_{3}\right): \delta 10.84(\mathrm{~s}, 1 \mathrm{H}), 7.78(\mathrm{~d}, J=8.9 \mathrm{~Hz}, 1 \mathrm{H}), 7.58(\mathrm{~s}, 1 \mathrm{H}), 7.21(\mathrm{dd}, J=19.2,9.7$ $\mathrm{Hz}, 3 \mathrm{H}), 7.07$ (t, $J=9.9 \mathrm{~Hz}, 2 \mathrm{H}), 5.09(\mathrm{~s}, 1 \mathrm{H}), 4.44(\mathrm{~s}, 1 \mathrm{H}), 4.01-3.71(\mathrm{~m}, 2 \mathrm{H}), 3.60-3.30(\mathrm{~m}, 2 \mathrm{H})$, $2.46(\mathrm{~s}, 3 \mathrm{H}), 1.19$ (t, $J=6.9 \mathrm{~Hz}, 3 \mathrm{H}), 0.47(\mathrm{t}, J=6.8 \mathrm{~Hz}, 3 \mathrm{H}) ;{ }^{31} \mathrm{P}$ NMR $\left(162 \mathrm{MHz}, \mathrm{CDCl}_{3}\right) \delta$ : $20.6(\mathrm{~s}) ;{ }^{13} \mathrm{C}\left\{{ }^{1} \mathrm{H}\right\} \operatorname{NMR}\left(101 \mathrm{MHz}, \mathrm{CDCl}_{3}\right): \delta 157.8,151.8,147.5,133.4,131.8,130.6,129.2$, $127.1,124.0,123.6,120.7,120.6,120.1,117.9,112.2,63.0,62.7,21.2,15.8,14.9$; HRMS (ESITOF) $\mathrm{m} / \mathrm{z}$ : $[\mathrm{M}+\mathrm{H}]^{+}$calcd for $\left(\mathrm{C}_{21} \mathrm{H}_{24} \mathrm{O}_{6} \mathrm{P}\right), 403.1305$; found, 403.1298. Enantiometric excess: 95\%, determined by HPLC (FLM Chiral NY (2) column, hexane/EtOH 75:25, flow rate $\left.0.5 \mathrm{~mL} / \mathrm{min}, \mathrm{t}_{\text {major }}=14.47 \mathrm{~min}, \mathrm{t}_{\text {minor }}=12.09 \mathrm{~min}, \lambda=210 \mathrm{~nm}\right)$.

Diethyl (2',3,6-trihydroxy-4',6'-dimethoxy-[1,1'-biphenyl]-2-yl)phosphonate $(4 \mathrm{cn}) . \quad$ The product was isolated by flash chromatography $(\mathrm{PE} / \mathrm{EA}=3: 1)$ as a white soild $(90.2 \mathrm{mg}, 76 \%)$, $\mathrm{Rf}$ $=0.14(\mathrm{PE} / \mathrm{EA}=2: 1)$, m.p. $159.1-160.8{ }^{\circ} \mathrm{C} ;[\alpha]_{D}^{20}=-35.0\left(\mathrm{c}=0.5, \mathrm{CH}_{2} \mathrm{Cl}_{2}\right) ;{ }^{1} \mathrm{H}$ NMR $(400 \mathrm{MHz}$, $\left.\mathrm{CDCl}_{3}\right): \delta 10.78(\mathrm{~s}, 1 \mathrm{H}), 7.15(\mathrm{~d}, J=9.0 \mathrm{~Hz}, 1 \mathrm{H}), 6.96(\mathrm{dd}, J=8.9,7.3 \mathrm{~Hz}, 1 \mathrm{H}), 6.22(\mathrm{~d}, J=2.1$ $\mathrm{Hz}, 1 \mathrm{H}), 6.14(\mathrm{~d}, J=2.1 \mathrm{~Hz}, 1 \mathrm{H}), 4.93(\mathrm{~s}, 1 \mathrm{H}), 4.61(\mathrm{~s}, 1 \mathrm{H}), 4.06-3.93(\mathrm{~m}, 2 \mathrm{H}), 3.91-3.82(\mathrm{~m}$, 2H), $3.82(\mathrm{~s}, 3 \mathrm{H}), 3.68(\mathrm{~s}, 3 \mathrm{H}), 1.26-1.14(\mathrm{~m}, 6 \mathrm{H}) ;{ }^{31} \mathrm{P}$ NMR $\left(162 \mathrm{MHz}, \mathrm{CDCl}_{3}\right) \delta: 21.4(\mathrm{~s})$; ${ }^{13} \mathrm{C}\left\{{ }^{1} \mathrm{H}\right\}$ NMR $\left(101 \mathrm{MHz}, \mathrm{CDCl}_{3}\right): \delta 162.6,159.4,157.6,157.5,156.3,147.6,147.4,122.98$, $122.96,120.2,120.1,118.6,118.5,110.2,108.4,101.0,100.9,93.5,91.5,62.89,62.86,62.83$, 62.81, 55.5, 55.4, 16.0, 15.9, 15.84, 15.77; HRMS (ESITOF) $\mathrm{m} / \mathrm{z}:[\mathrm{M}+\mathrm{H}]^{+}$calcd for $\left(\mathrm{C}_{18} \mathrm{H}_{24} \mathrm{O}_{8} \mathrm{P}\right), 399.1203$; found, 399.1200. Enantiometric excess: $74 \%$, determined by HPLC (FLM Chiral NY (2) column, hexane/EtOH 40:60, flow rate $0.5 \mathrm{~mL} / \mathrm{min}$, $\mathrm{t}_{\text {major }}=9.87 \mathrm{~min}, \mathrm{t}_{\text {minor }}=15.16$ $\min , \lambda=210 \mathrm{~nm})$.

\section{7、Preparative scale synthesis of $4 \mathrm{ca}$}

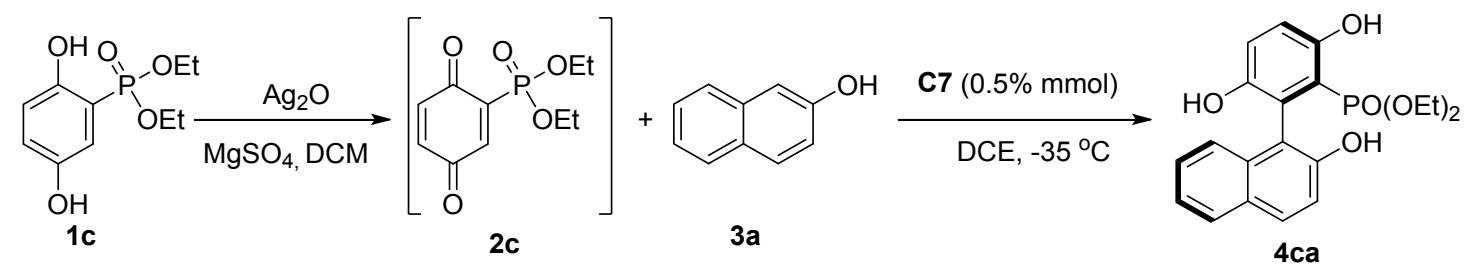

To a mixture of $1 \mathrm{c}\left(2.21 \mathrm{~g}, 9.0 \mathrm{mmol}, 1.0\right.$ equiv), $\mathrm{Ag}_{2} \mathrm{O}$ (2.50 g, $10.80 \mathrm{mmol}, 1.2$ equiv) and $\mathrm{MgSO}_{4}\left(3.25 \mathrm{~g}, 27.0 \mathrm{mmol}, 3.0\right.$ equiv) in DCM $(180 \mathrm{~mL})$ for $2 \mathrm{~h}$ at $25^{\circ} \mathrm{C}$, the mixture was filtered and the filtrate was concentrated in vacuo to give $\mathbf{2 c}$, which was used directly. Then, $2 \mathbf{c}, \mathbf{3 a}$ (1.56 $\mathrm{g}, 10.80 \mathrm{mmol}, 1.2$ equiv), $\mathbf{C} 7$ (30.0 $\mathrm{mg}, 0.045 \mathrm{mmol}, 0.5 \mathrm{~mol} \%)$ was added in DCE (180 mL) at $-35{ }^{\circ} \mathrm{C}$. After stirred at $-35{ }^{\circ} \mathrm{C}$ for $44.5 \mathrm{~h}$, the mixture was concentrated and purified by silica gel column chromatography ( $\mathrm{PE} / \mathrm{EA}=5 / 1)$ to afford pure product $\mathbf{4} \mathbf{c a}(3.29 \mathrm{~g}, 94 \%$ yield, $94 \% \mathrm{ee})$. 


\section{8、Preparation of chiral phosphine 6 from $4 \mathrm{ca}$}

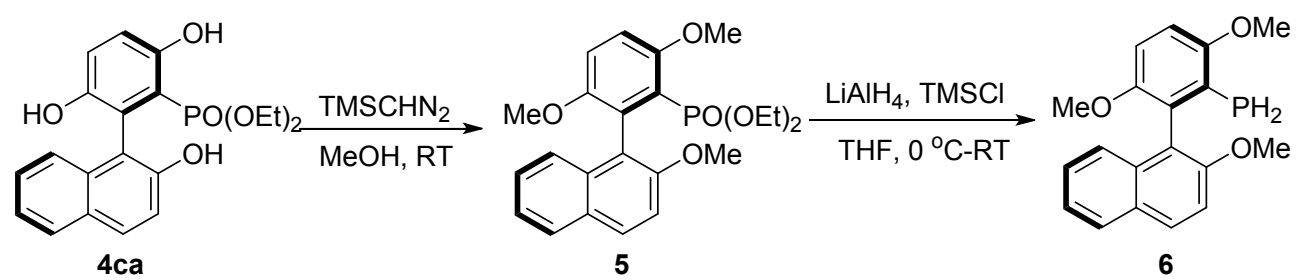

Step 1: Diethyl (3,6-dimethoxy-2-(2-methoxynaphthalen-1-yl)phenyl)phosphonate (5): To a solution of $4 \mathbf{c a}\left(0.5 \mathrm{~g}, 1.29 \mathrm{mmol}, 1.0\right.$ equiv) in $\mathrm{MeOH}(10 \mathrm{~mL})$ was added $\mathrm{TMSCHN}_{2}(2.0 \mathrm{M}$ in hexane, $15 \mathrm{~mL}, 29.6 \mathrm{mmol}, 23.0$ equiv) at $0{ }^{\circ} \mathrm{C}$. The mixture was stirred for $5 \mathrm{~min}$ at $0{ }^{\circ} \mathrm{C}$. Subsequently, the mixture was stirred for $24 \mathrm{~h}$ at $25^{\circ} \mathrm{C}$. The mixture was concentrated to remove the solvents $\left(33{ }^{\circ} \mathrm{C}\right)$. The residue was purified by column chromatography $\left(\mathrm{CH}_{2} \mathrm{Cl}_{2} / \mathrm{MeOH}=\right.$ $100 / 1-40 / 1)$ to give $5\left(527.3 \mathrm{mg}, 95 \%\right.$ yield, $95 \%$ ee) as a white soild. $\mathrm{Rf}=0.38\left(\mathrm{CH}_{2} \mathrm{Cl}_{2} / \mathrm{MeOH}\right.$ $=20 / 1)$, m.p. $114.4-116.5^{\circ} \mathrm{C} ;[\alpha]_{D}^{20}=-7.5\left(\mathrm{c}=0.5, \mathrm{CH}_{2} \mathrm{Cl}_{2}\right) ;{ }^{1} \mathrm{H} \mathrm{NMR}\left(400 \mathrm{MHz}, \mathrm{CDCl}_{3}\right): \delta 7.85$ $(\mathrm{d}, J=9.0 \mathrm{~Hz}, 1 \mathrm{H}), 7.81-7.74(\mathrm{~m}, 1 \mathrm{H}), 7.34-7.24(\mathrm{~m}, 3 \mathrm{H}), 7.19(\mathrm{dd}, J=9.1,5.5 \mathrm{~Hz}, 2 \mathrm{H})$, 7.10-7.01 (m, 1H), $3.93(\mathrm{~s}, 3 \mathrm{H}), 3.82(\mathrm{~s}, 3 \mathrm{H}), 3.81-3.71(\mathrm{~m}, 2 \mathrm{H}), 3.70-3.60(\mathrm{~m}, 1 \mathrm{H}), 3.51(\mathrm{~s}, 3 \mathrm{H})$, 3.43-3.36 (m, 1H), $0.87(\mathrm{q}, J=7.3 \mathrm{~Hz}, 6 \mathrm{H}) ;{ }^{31} \mathrm{P}$ NMR $\left(162 \mathrm{MHz}, \mathrm{CDCl}_{3}\right) \delta: 16.0(\mathrm{~s}) ;{ }^{13} \mathrm{C}\left\{{ }^{1} \mathrm{H}\right\}$ NMR $\left(151 \mathrm{MHz}, \mathrm{CDCl}_{3}\right): \delta 156.63,156.62,153.8,152.2,152.0,133.8,131.3,131.2,128.9$, $128.7,127.7,126.1,124.6,123.2,120.74,120.71,118.6,117.37,117.36,117.31,113.4,112.1$, $112.0,62.4,62.3,62.10,62.06,56.9,56.6,56.5,15.73,15.68,15.64 ;$ HRMS (ESITOF) m/z: [M + $\mathrm{H}]^{+}$calcd for $\left(\mathrm{C}_{23} \mathrm{H}_{28} \mathrm{O}_{6} \mathrm{P}\right), 431.1618$; found, 431.1621 . Enantiometric excess: $95 \%$, determined by HPLC (FLM Chiral ND (2) column, hexane/EtOH 90:10, flow rate $0.5 \mathrm{~mL} / \mathrm{min}$, $\mathrm{t}_{\text {major }}=18.63 \mathrm{~min}$, $\mathrm{t}_{\text {minor }}=15.73 \mathrm{~min}, \lambda=210 \mathrm{~nm}$ ).

Step 2: (3,6-Dimethoxy-2-(2-methoxynaphthalen-1-yl)phenyl)phosphine (6): Under $\mathrm{N}_{2}$ protection, to a $50 \mathrm{~mL}$ round-bottomed flask were added Lithium aluminum hydride $(352.7 \mathrm{mg}$, 9.29 mmol, 8.0 equiv) and $5 \mathrm{~mL}$ of anhydrous THF, and the mixture was cooled to $0{ }^{\circ} \mathrm{C}$. Chlorotrimethylsilane (1.01 g, $9.29 \mathrm{mmol}, 8.0$ equiv) was added dropwise to the LAH solution. This solution was allowed to stir at $0{ }^{\circ} \mathrm{C}$ for 30 min then diethyl (3,6-dimethoxy-2-(2-methoxynaphthalen-1-yl)phenyl)phosphonate (5) (0.5 g, $1.16 \mathrm{mmol}, 1.0$ equiv) in $10 \mathrm{~mL}$ of anhydrous THF added dropwise to the LAH/TMSCl over $30 \mathrm{~min}$. The reaction was allowed to proceed overnight with slow warming to the room temperature. The reaction mixture was cooled to $0{ }^{\circ} \mathrm{C}$. The reaction was quenched by adding EtOAc $(5 \mathrm{~mL})$, and aqueous hydrochloric acid $(1 \mathrm{M}, 10 \mathrm{~mL})$. This mixture was allowed to stir for 30 min under an atmosphere of nitrogen at $0{ }^{\circ} \mathrm{C}$. The solution was transferred to a separatory funnel and the layers were separated. The aqueous layer was extracted with EtOAc $(2 \times 10 \mathrm{~mL})$. The combined organics layers were washed with a saturated solution of $\mathrm{NaCl}(30 \mathrm{~mL})$. The organic solution was dried over anhydrous $\mathrm{Na}_{2} \mathrm{SO}_{4}$, filtered, and concentrated in vacuo to give (3,6-Dimethoxy-2-(2-methoxynaphthalen-1-yl)phenyl)phosphine (6) as yellowish solid (385.9 $\mathrm{mg},>99 \%$ yield, $84 \%$ ee). This material was used without further purification. $\mathrm{Rf}=0.33(\mathrm{PE} / \mathrm{EA}$ $=5 / 1)$, m.p. $162.0-164.2{ }^{\circ} \mathrm{C} ;[\alpha]_{D}^{20}=-68.0\left(\mathrm{c}=0.5, \mathrm{CH}_{2} \mathrm{Cl}_{2}\right) ;{ }^{1} \mathrm{H}$ NMR $\left(400 \mathrm{MHz}, \mathrm{CDCl}_{3}\right): \delta 7.92$ $(\mathrm{d}, J=9.0 \mathrm{~Hz}, 1 \mathrm{H}), 7.83(\mathrm{dd}, J=5.4,4.0 \mathrm{~Hz}, 1 \mathrm{H}), 7.39(\mathrm{~d}, J=9.0 \mathrm{~Hz}, 1 \mathrm{H}), 7.36-7.28(\mathrm{~m}, 2 \mathrm{H})$, 7.25-7.19 (m, 1H), $6.93(\mathrm{dt}, J=8.9,5.9 \mathrm{~Hz}, 2 \mathrm{H}), 3.89(\mathrm{~d}, J=14.4 \mathrm{~Hz}, 6 \mathrm{H}), 3.58(\mathrm{~s}, 3 \mathrm{H}) ;{ }^{31} \mathrm{P}$ NMR $\left(162 \mathrm{MHz}, \mathrm{CDCl}_{3}\right) \delta:-154.8(\mathrm{~s}) ;{ }^{13} \mathrm{C}\left\{{ }^{1} \mathrm{H}\right\}$ NMR $\left(151 \mathrm{MHz}, \mathrm{CDCl}_{3}\right): \delta 154.53,154.49,154.0$, 
$152.26,152.25,132.9,130.22,130.16,129.5,129.1,128.04,127.96,126.5,124.6,123.5,122.1$, 122.0, 121.2, 121.1, 113.8, 111.5, 109.5, 56.8, 56.6, 56.0; HRMS (ESITOF) $\mathrm{m} / \mathrm{z}:[\mathrm{M}+\mathrm{H}]^{+}$calcd for $\left(\mathrm{C}_{19} \mathrm{H}_{20} \mathrm{O}_{3} \mathrm{P}\right), 327.1145$; found, 327.1140. Enantiometric excess: $84 \%$, determined by HPLC (FLM Chiral MD (2) column, hexane/EtOH 70:30, flow rate $0.5 \mathrm{~mL} / \mathrm{min}, \mathrm{t}_{\text {major }}=9.44 \mathrm{~min}, \mathrm{t}_{\text {minor }}=$ $8.94 \min , \lambda=210 \mathrm{~nm})$.

\section{9、Typical Experimental Procedure for the Synthesis of Compounds $7 \mathrm{a}$ and $7 \mathrm{~b}$.}

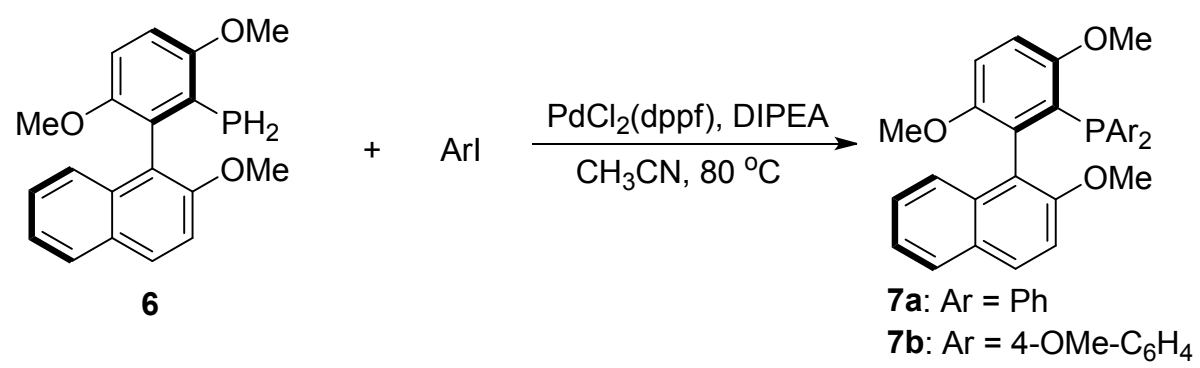

Under $\mathrm{N}_{2}$ protection, to a $50 \mathrm{~mL}$ round-bottomed flask were added $\mathrm{PdCl}_{2}$ (dppf) $(40.4 \mathrm{mg}, 0.055$ mmol, 0.1 equiv), diisopropylethylamine (285.2 $\mathrm{mg}, 2.21 \mathrm{mmol}, 4.0$ equiv), aryl iodide (1.65 mmol, 3.0 equiv) then (3,6-Dimethoxy-2-(2-methoxynaphthalen-1-yl)phenyl)phosphine (6) (180.0 $\mathrm{mg}, 0.552 \mathrm{mmol}, 1.0$ equiv) in $10 \mathrm{~mL}$ acetonitrile was added, and the mixture was stirred at $80{ }^{\circ} \mathrm{C}$ for $2 \mathrm{~h}$. After completion of the reaction, the mixture was concentrated and purified by silica gel flash chromatography using PE and EA (5:1) as an eluent.

(3,6-Dimethoxy-2-(2-methoxynaphthalen-1-yl)phenyl)diphenylphosphine (7a): $\operatorname{Rf}=0.33$ $(\mathrm{PE} / \mathrm{EA}=5 / 1)$, white soild, $87.5 \mathrm{mg}, 33 \%$ yield, $90 \%$ ee, m.p. $195.1-197.2{ }^{\circ} \mathrm{C} ;[\alpha]_{D}^{20}=+14.0$ $\left(\mathrm{c}=0.5, \mathrm{CH}_{2} \mathrm{Cl}_{2}\right) ;{ }^{1} \mathrm{H}$ NMR $\left(400 \mathrm{MHz}, \mathrm{CDCl}_{3}\right): \delta 7.90(\mathrm{~d}, J=9.0 \mathrm{~Hz}, 1 \mathrm{H}), 7.86-7.79(\mathrm{~m}, 1 \mathrm{H})$, 7.36-7.20 (m, 9H), 7.15-7.12 (m, 6H), $6.91(\mathrm{~d}, J=8.9 \mathrm{~Hz}, 1 \mathrm{H}), 3.59$ (s, 3H), 3.50 (s, 3H), $3.29(\mathrm{~s}$, 3H); ${ }^{31} \mathrm{P}$ NMR (162 MHz, $\left.\mathrm{CDCl}_{3}\right) \delta: 13.0(\mathrm{~s}) ;{ }^{13} \mathrm{C}\left\{{ }^{1} \mathrm{H}\right\} \mathrm{NMR}\left(151 \mathrm{MHz}, \mathrm{CDCl}_{3}\right): \delta 156.5,156.4$, $154.27,154.25,152.1,152.0,138.3,138.2$, 137.1, 137.0, 134.9, 133.71, 133.70, 132.6, 132.4, $132.3,132.2,128.0,127.5,127.4,127.21,127.17,127.0,126.9,126.0,125.1,124.8,123.2,121.8$, 121.7, 114.8, 112.7, 112.0, 56.7, 55.7, 55.2; HRMS (ESITOF) $\mathrm{m} / \mathrm{z}:[\mathrm{M}+\mathrm{H}]^{+}$calcd for $\left(\mathrm{C}_{31} \mathrm{H}_{28} \mathrm{O}_{3} \mathrm{P}\right), 479.1771$; found, 479.1776 . Enantiometric excess: $90 \%$, determined by HPLC (FLM Chiral ND (2) column, hexane/EtOH 70:30, flow rate $0.5 \mathrm{~mL} / \mathrm{min}, \mathrm{t}_{\text {major }}=7.83 \mathrm{~min}, \mathrm{t}_{\text {minor }}=8.60$ $\min , \lambda=210 \mathrm{~nm})$.

(3,6-Dimethoxy-2-(2-methoxynaphthalen-1-yl)phenyl)bis(4-methoxyphenyl)phosphine (7b): $\mathrm{Rf}=0.21(\mathrm{PE} / \mathrm{EA}=5 / 1)$, yellowish soild, $146.5 \mathrm{mg}, 44 \%$ yield, $90 \%$ ee, m.p. $137.7-139.7{ }^{\circ} \mathrm{C} ;[\alpha]$ ${ }_{D}^{20}=+22.0(\mathrm{c}=0.5, \mathrm{EA}) ;{ }^{1} \mathrm{H}$ NMR $\left(400 \mathrm{MHz}, \mathrm{CDCl}_{3}\right): \delta 7.88(\mathrm{~d}, J=9.0 \mathrm{~Hz}, 1 \mathrm{H}), 7.84-7.78(\mathrm{~m}$, 1H), 7.34-7.18 (m, 6H), 7.14-7.01 (m, 3H), $6.91(\mathrm{~d}, J=8.9 \mathrm{~Hz}, 1 \mathrm{H}), 6.79(\mathrm{~d}, J=7.9 \mathrm{~Hz}, 2 \mathrm{H}), 6.68$ $(\mathrm{d}, J=8.3 \mathrm{~Hz}, 2 \mathrm{H}), 3.76(\mathrm{~d}, J=22.6 \mathrm{~Hz}, 6 \mathrm{H}), 3.55(\mathrm{~d}, J=12.4 \mathrm{~Hz}, 6 \mathrm{H}), 3.37(\mathrm{~s}, 3 \mathrm{H}) ;{ }^{31} \mathrm{P}$ NMR $\left(162 \mathrm{MHz}, \mathrm{CDCl}_{3}\right) \delta: 14.7(\mathrm{~s}) ;{ }^{13} \mathrm{C}\left\{{ }^{1} \mathrm{H}\right\} \mathrm{NMR}\left(151 \mathrm{MHz}, \mathrm{CDCl}_{3}\right): \delta 156.43,156.41,154.24$, $154.23,152.1,152.0,134.4,134.3,134.2,133.7,133.6,129.1,128.6,127.9,126.0,125.1,123.1$, $114.6,113.22,113.17,113.0,112.91,112.85,112.0,56.7,55.9,55.4,55.1,55.0$; HRMS (ESITOF) $\mathrm{m} / \mathrm{z}:[\mathrm{M}+\mathrm{H}]^{+}$calcd for $\left(\mathrm{C}_{33} \mathrm{H}_{32} \mathrm{O}_{5} \mathrm{P}\right), 539.1982$; found, 539.1975. Enantiometric excess: $90 \%$, determined by HPLC (FLM Chiral NX (2) column, hexane/EtOH 70:30, flow rate $0.5 \mathrm{~mL} / \mathrm{min}, \mathrm{t}_{\text {major }}=14.03 \mathrm{~min}, \mathrm{t}_{\text {minor }}=18.23 \mathrm{~min}, \lambda=210 \mathrm{~nm}$ ). 


\section{0、Typical Experimental Procedure for the Synthesis of Compound 8.}

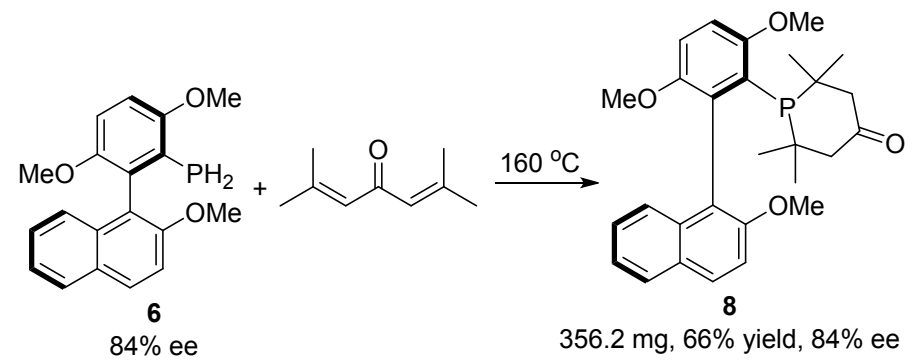

1-(3,6-Dimethoxy-2-(2-methoxynaphthalen-1-yl)phenyl)-2,2,6,6-tetramethylphosphinan-4-on

e (8): 2,6-Dimethyl-2,5-heptadien-4-one $(1.0 \mathrm{~mL}, 6.43 \mathrm{mmol}, 5.54$ equiv) and (3,6-Dimethoxy-2-(2-methoxynaphthalen-1-yl)phenyl)phosphine (6) (379.0 mg, $1.16 \mathrm{mmol}, 1.0$ equiv) were charged to a $10-\mathrm{mL}$ sealed tube with a magnetic stir bar. The tube was purged with nitrogen and sealed with a Teflon stopcock. The mixture was stirred at $160{ }^{\circ} \mathrm{C}$ for $21 \mathrm{~h}$ and the contents were allowed to cool to room temperature. The mixture was purified by column chromatography ( $\mathrm{PE} / \mathrm{EA}=5 / 1)$ to give $8(356.2 \mathrm{mg}, 66 \%$ yield, $84 \%$ ee) as a white soild. $\mathrm{Rf}=$ 0.24 (PE/EA $=5 / 1)$, m.p. $199.1-200.3{ }^{\circ} \mathrm{C} ;[\alpha]_{D}^{20}=+1.2\left(\mathrm{c}=0.5\right.$, EA); ${ }^{1} \mathrm{H}$ NMR $\left(400 \mathrm{MHz}, \mathrm{CDCl}_{3}\right)$ : $\delta 7.89(\mathrm{~d}, J=9.0 \mathrm{~Hz}, 1 \mathrm{H}), 7.82-7.76(\mathrm{~m}, 1 \mathrm{H}), 7.34-7.21(\mathrm{~m}, 3 \mathrm{H}), 7.09(\mathrm{~d}, J=9.0 \mathrm{~Hz}, 2 \mathrm{H}), 6.96(\mathrm{~d}$, $J=8.9 \mathrm{~Hz}, 1 \mathrm{H}), 3.85(\mathrm{~s}, 6 \mathrm{H}), 3.54(\mathrm{~s}, 3 \mathrm{H}), 3.06(\mathrm{dd}, J=19.8,13.1 \mathrm{~Hz}, 2 \mathrm{H}), 2.12(\mathrm{ddd}, J=41.2$, 13.6, $5.6 \mathrm{~Hz}, 2 \mathrm{H}), 1.11-0.97(\mathrm{~m}, 6 \mathrm{H}), 0.81(\mathrm{~d}, J=9.0 \mathrm{~Hz}, 3 \mathrm{H}), 0.69(\mathrm{~d}, J=22.3 \mathrm{~Hz}, 3 \mathrm{H}) ;{ }^{31} \mathrm{P}$ $\operatorname{NMR}\left(162 \mathrm{MHz}, \mathrm{CDCl}_{3}\right) \delta: 8.9(\mathrm{~s}) ;{ }^{13} \mathrm{C}\left\{{ }^{1} \mathrm{H}\right\} \mathrm{NMR}\left(151 \mathrm{MHz}, \mathrm{CDCl}_{3}\right): \delta 155.07,155.05,153.39$, $153.37,152.53,152.46,137.7,137.4,133.8,133.7,128.8,128.6,127.8,126.3,126.0,125.52$, $125.49,122.9,122.0,114.1,112.5,109.1,56.7,55.7,54.7,54.4,54.2,35.1,34.94,34.88,34.7$, 34.4, 34.1, 33.8, 33.5, 29.4, 28.8; HRMS (ESITOF) $\mathrm{m} / \mathrm{z}$ : $[\mathrm{M}+\mathrm{H}]^{+}$calcd for $\left(\mathrm{C}_{28} \mathrm{H}_{34} \mathrm{O}_{4} \mathrm{P}\right)$, 465.2189; found, 465.2185. Enantiometric excess: 84\%, determined by HPLC (FLM Chiral MD (2) column, hexane $/ \mathrm{EtOH} 90: 10$, flow rate $0.5 \mathrm{~mL} / \mathrm{min}, \mathrm{t}_{\text {major }}=12.17 \mathrm{~min}, \mathrm{t}_{\text {minor }}=11.55 \mathrm{~min}, \lambda=$ $210 \mathrm{~nm})$.

\section{Application of atropisomeric phosphine $7 \mathrm{a}^{2}$}

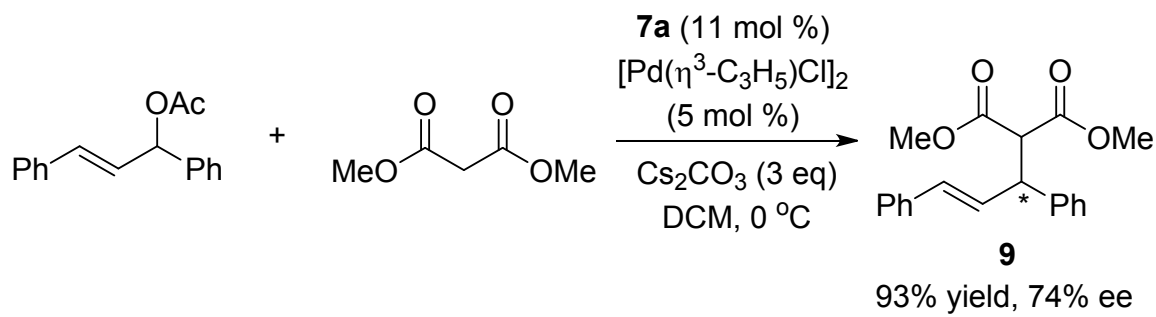

To a mixture of chiral ligand $7 \mathbf{a}(13.1 \mathrm{mg}, 0.0275 \mathrm{mmol}),\left[\mathrm{Pd}\left(\eta^{3}-\mathrm{C}_{3} \mathrm{H}_{5}\right) \mathrm{Cl}\right]_{2}(4.6 \mathrm{mg}, 0.0125$ $\mathrm{mmol})$ and $\mathrm{Cs}_{2} \mathrm{CO}_{3}(244.4 \mathrm{mg}, 0.75 \mathrm{mmol})$ in $\mathrm{CH}_{2} \mathrm{Cl}_{2}(2 \mathrm{~mL})$ were added 1,3-diphenylallyl acetate $(63.1 \mathrm{mg}, 0.25 \mathrm{mmol})$ at room temperature under argon atmosphere. After $30 \mathrm{~min}$, dimethyl malonate $(99.1 \mathrm{mg}, 0.75 \mathrm{mmol})$ was added at $0{ }^{\circ} \mathrm{C}$, and the reaction mixture then was stirred at 0 ${ }^{\circ} \mathrm{C}$ for $12 \mathrm{~h}$. The reaction mixture was quenched saturated ammonium chloride aqueous solution 
$(10 \mathrm{~mL})$, and the product was extracted into $\mathrm{CH}_{2} \mathrm{Cl}_{2}(3 \times 10 \mathrm{~mL})$, The organic layer was washed with brine and dried over $\mathrm{Na}_{2} \mathrm{SO}_{4}$. The filtrate was concentrated and purified by column chromatography $(\mathrm{PE} / \mathrm{EA}=15 / 1)$ to afford $9(75.6 \mathrm{mg}, 93 \%$ yield, $74 \%$ ee $)$ as colorless oil. $\mathrm{Rf}=$ $0.24(\mathrm{PE} / \mathrm{EA}=5 / 1),{ }^{1} \mathrm{H}$ NMR $\left(400 \mathrm{MHz}, \mathrm{CDCl}_{3}\right): \delta 7.36-7.17(\mathrm{~m}, 10 \mathrm{H}), 6.49(\mathrm{~d}, J=15.8 \mathrm{~Hz}$, $1 \mathrm{H}), 6.34(\mathrm{dd}, J=15.7,8.7 \mathrm{~Hz}, 1 \mathrm{H}), 4.36-4.23(\mathrm{~m}, 1 \mathrm{H}), 3.97(\mathrm{~d}, J=10.9,1 \mathrm{H}), 3.71(\mathrm{~s}, 3 \mathrm{H}), 3.53$ (s, 3H); ${ }^{13} \mathrm{C}\left\{{ }^{1} \mathrm{H}\right\}$ NMR $\left(101 \mathrm{MHz}, \mathrm{CDCl}_{3}\right): \delta 168.2,167.7,140.1,136.8,131.8,129.1,128.7$, 128.4, 127.8, 127.5, 127.1, 126.3, 57.6, 52.6, 52.4, 49.1; HRMS (ESITOF) $\mathrm{m} / \mathrm{z}:[\mathrm{M}+\mathrm{Na}]^{+}$calcd for $\left(\mathrm{C}_{20} \mathrm{H}_{20} \mathrm{NaO}_{4}\right), 347.1254$; found, 347.1249. Enantiometric excess: $74 \%$, determined by HPLC (FLM Chiral ND (2) column, hexane $/$ EtOH 30:70, flow rate $0.5 \mathrm{~mL} / \mathrm{min}$, $\mathrm{t}_{\text {major }}=17.62 \mathrm{~min}$, $\mathrm{t}_{\text {minor }}$ $=27.16 \mathrm{~min}, \lambda=210 \mathrm{~nm})$.

\section{2、X-ray Single Crystal Structure Analysis of 4ca}

The clear light colourless block-shaped crystals suitable for X-ray structure analyses were obtained by gas-vapor diffusion in EA/ $\mathrm{n}$-hexane at $25^{\circ} \mathrm{C}$.

Suitable block crystal $(0.3 \mathrm{~mm} \times 0.3 \mathrm{~mm} \times 0.2 \mathrm{~mm})$ of $4 \mathbf{c a}$ was selected, attached to a glass fiber, and mounted on a Bruker Smart APEX-II CCD system with graphite-monochromated $\mathrm{Cu} \mathrm{K \alpha}$ radiation ( $\lambda=1.54184 \AA$ ) using $\square$ and $\omega$ scans for data collection at $100 \mathrm{~K}$. Cell parameters were determined using SMART software and refined using SAINT Plus. ${ }^{3}$ Data reduction and absorption corrections were made via SADABS. The structure was solved via direct methods ${ }^{4}$ using SHELXS and refined via least squares using SHELXL. ${ }^{5}$ Hydrogen atoms riding on carbon atoms were placed in calculated positions and refined with isotropic thermal parameters. The positions of $\mathrm{H}$-atoms bonded to $\mathrm{N} / \mathrm{O}$ atoms were refined with anisotropic thermal parameters. Details of the data collection parameters and crystallographic information for $\mathbf{4 c a}$ were provided in Table S1.

Table S1. Crystal data and structure refinement for Compound 4ca

\begin{tabular}{|l|l|}
\hline Empirical formula & $\mathrm{C}_{20} \mathrm{H}_{21} \mathrm{O}_{6} \mathrm{P}$ \\
\hline Formula weight & 388.34 \\
\hline Temperature & $100 \mathrm{~K}$ \\
\hline Wavelength & $1.54184 \AA$ \\
\hline Crystal system & orthorhombic \\
\hline Space group & $P 2_{1} 2_{1} 2_{1}$ \\
\hline Unit cell dimensions & $a=7.99520(10) \AA \quad$ alpha $=90^{\circ}$ \\
& $b=12.80710(10) \AA \quad \begin{array}{r}\text { beta }=90^{\circ} \\
\text { gamma }=90^{\circ}\end{array}$ \\
\hline Volume & $c=17.7702(2) \AA \quad 1819.59(11) \AA 3$ \\
\hline$Z$ & 4 \\
\hline Calculated density & $1.381 \mathrm{Mg} / \mathrm{m}^{3}$ \\
\hline
\end{tabular}




\begin{tabular}{|l|l|}
\hline $\mathrm{F}(000)$ & 792 \\
\hline Crystal size & $0.3 \times 0.3 \times 0.2 \mathrm{~mm}^{3}$ \\
\hline Theta range for data collection & 4.255 to $75.943^{\circ}$ \\
\hline Limiting indices & $-10<=\mathrm{h}<=8,-15<=\mathrm{k}<=14,-22<=\mathrm{l}<=21$ \\
\hline Reflections collected & 9079 \\
\hline Independent reflections & $3679[\mathrm{R}($ int $)=0.0197]$ \\
\hline Max. and min. transmission & 1.00000 and 0.82510 \\
\hline Data / restraints / parameters & $3679 / 0 / 249$ \\
\hline Goodness-of-fit on $\mathrm{F}^{2}$ & 1.064 \\
\hline Final R indices [I $>2$ sigma(I)] & $\mathrm{R}_{1}=0.0263, w \mathrm{R}_{2}=0.0706$ \\
\hline $\mathrm{R}$ indices (all data) & $\mathrm{R}_{1}=0.0264, w \mathrm{R}_{2}=0.0707$ \\
\hline Largest diff. peak and hole & 0.325 and -0.308 e $\AA^{-3}$ \\
\hline
\end{tabular}

These data (CCDC 2105857) can be obtained free of charge from the Cambridge Crystallographic Data Centre via www.ccdc.cam.ac.uk/data_request/cif.

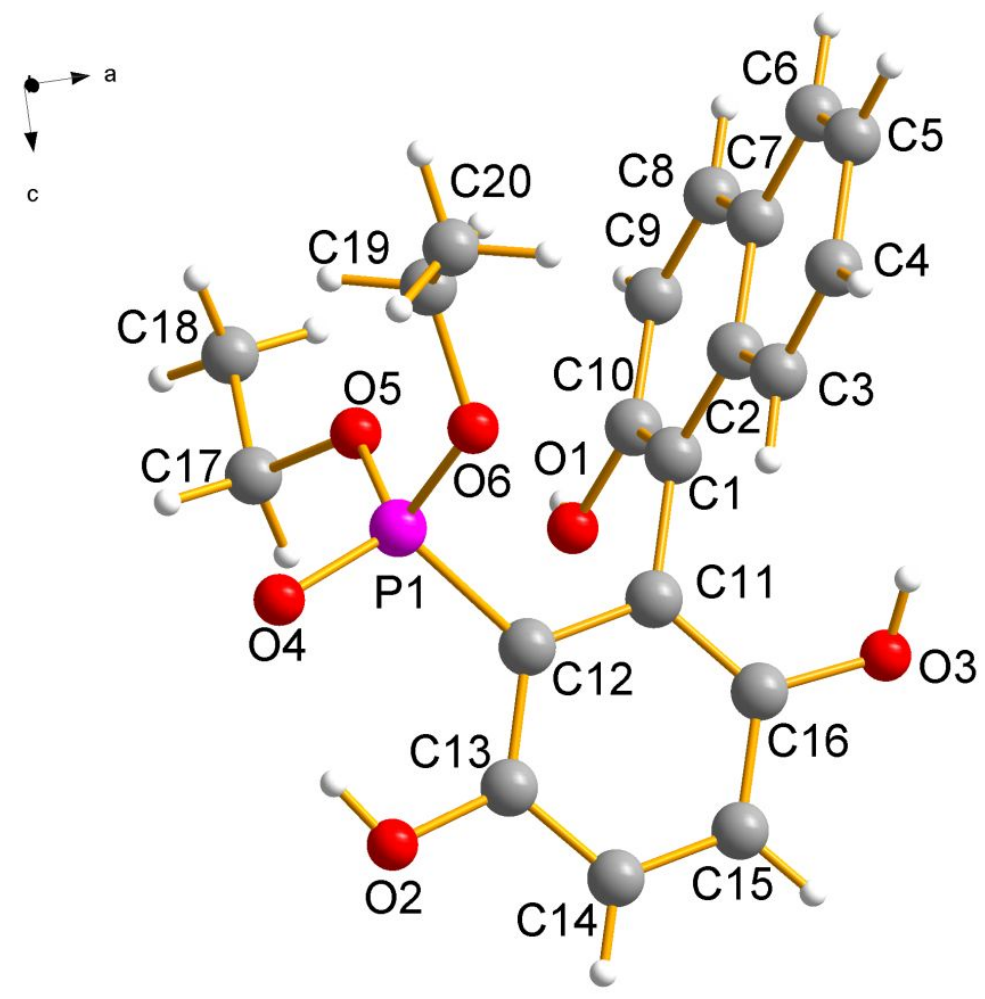

FIGURE S1. The crystal structure of 4ca by X-ray analysis. 


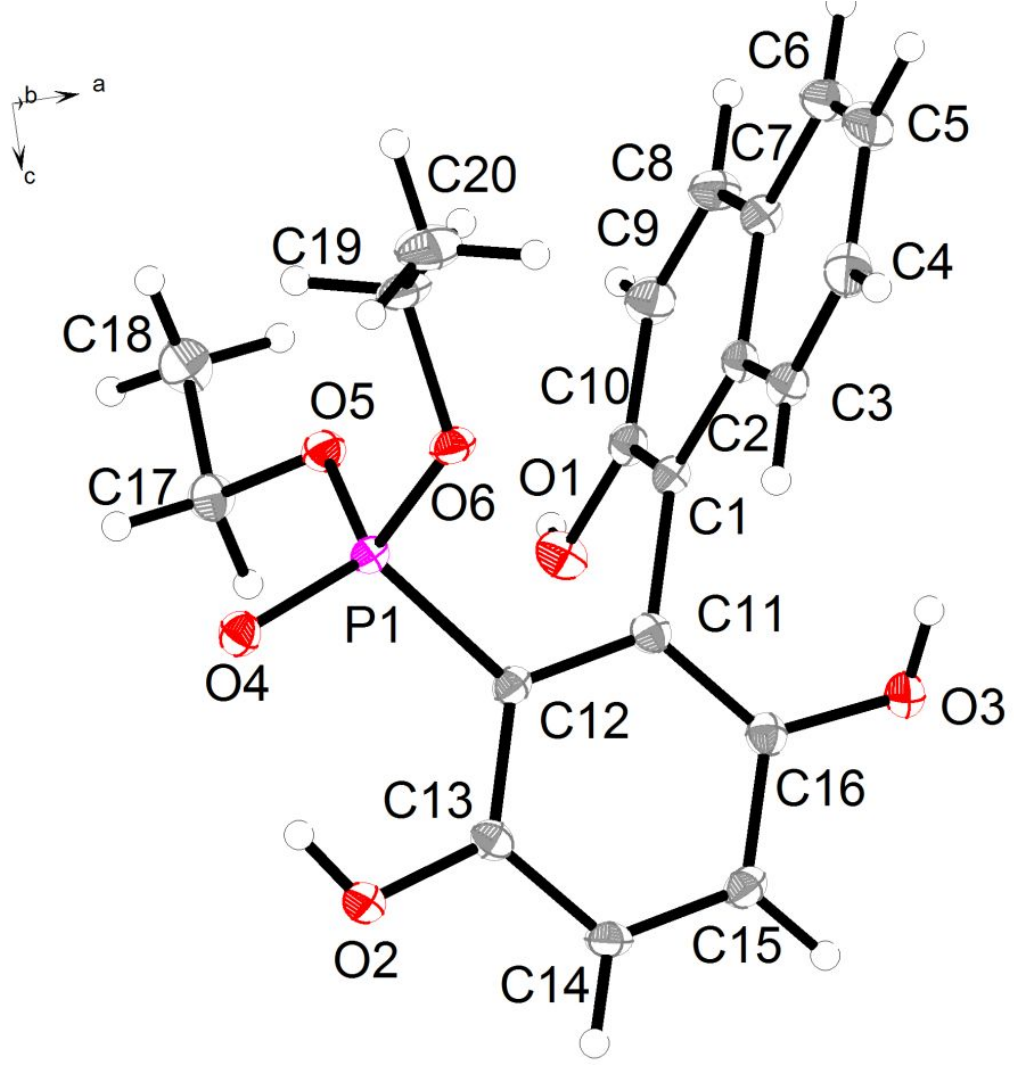

FIGURE S2. ORTEP plot (50\% probability ellipsoids) showing the molecule structure of 4ca.

\section{3、Referances:}

[1] Sun, G.-D.; Wang, Z.-H.; Deng, Z.-F.; Luo, Z.-H.; Zhang, J.-C. Development of novel C2-symmetrical [5]oxahelicenoid diols. Tetrahedron Lett., 2021, 71, 153026.

[2] Vyskočil, Š.; Smrčina, M.; Hanuš, V.; Polášek, M.; Kočovský, P. Derivatives of 2-Amino-2'-diphenylphosphino-1,1'-binaphthyl (MAP) and Their Application in Asymmetric Palladium(0)-Catalyzed Allylic Substitution. J. Org. Chem. 1998, 63, 7738-7748.

[3] SHELXTL, Version 5.10. Structure Determination Software Programs, Bruker Analytical X-ray Systems Inc., Madison, Wisconsin, USA, 2000.

[4] Dolomanov, O. V.; Bourhis, L. J.; Gildea, R. J.; Howard, J. A. K.; Puschmann, H. J. Appl. Crystallogr. 2009, 42, 339-341.

[5] Sheldrick, G. Acta Crystallogr. C Struct. Chem. 2015, 71, 3-8.

\section{4、 ${ }^{1} \mathrm{H}$ NMR, ${ }^{31} \mathrm{P}$ NMR and ${ }^{13} \mathrm{C}\{1 \mathrm{H}\}$ NMR spectra}

${ }^{1} \mathrm{H}$ NMR for compound 4 aa (400 MHz, DMSO- $\mathrm{d}_{6}$ ) 


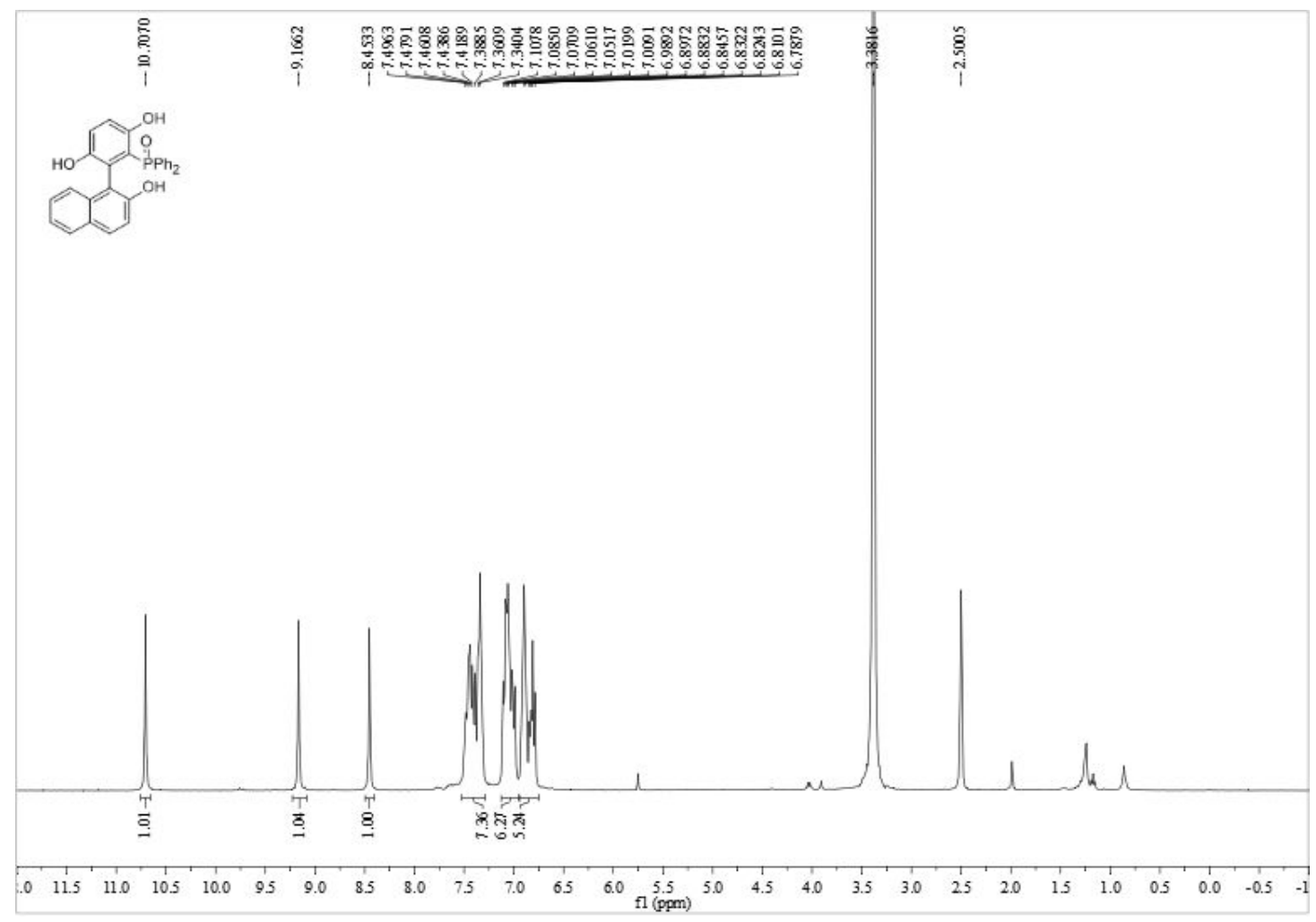

${ }^{31} \mathrm{P}$ NMR for compound 4aa (162 MHz, DMSO-d $\left.\mathrm{d}_{6}\right)$

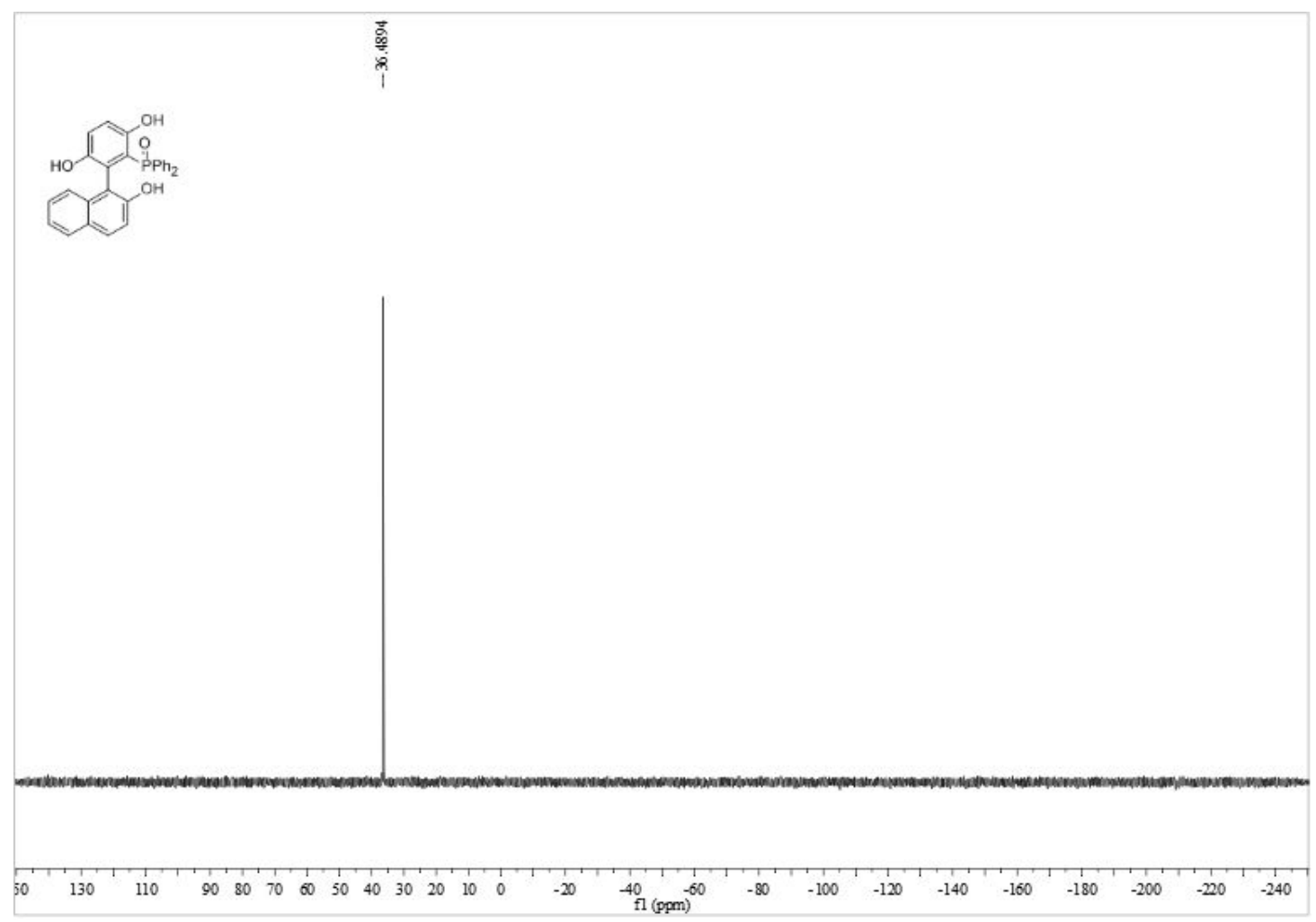

S-19 
${ }^{13} \mathrm{C}\left\{{ }^{1} \mathrm{H}\right\}$ NMR for compound 4aa (151 MHz, DMSO- $\mathrm{d}_{6}$ )

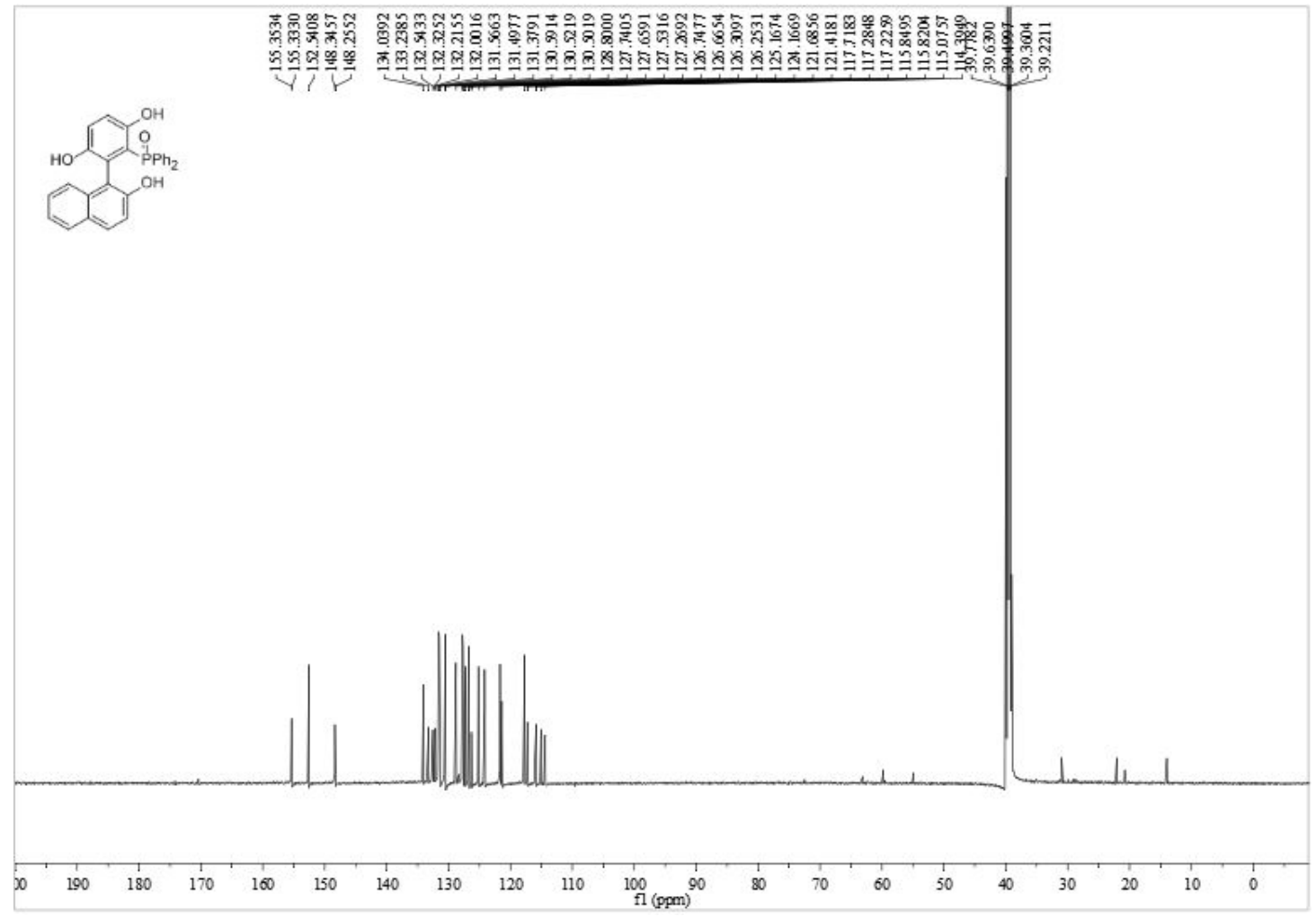

${ }^{1} \mathrm{H}$ NMR for compound $\mathbf{4 b a}\left(400 \mathrm{MHz}, \mathrm{CDCl}_{3}\right)$

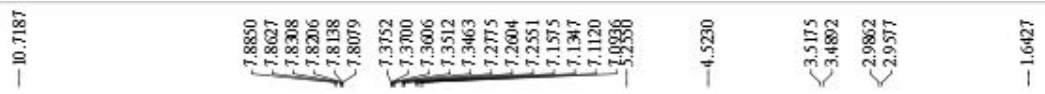
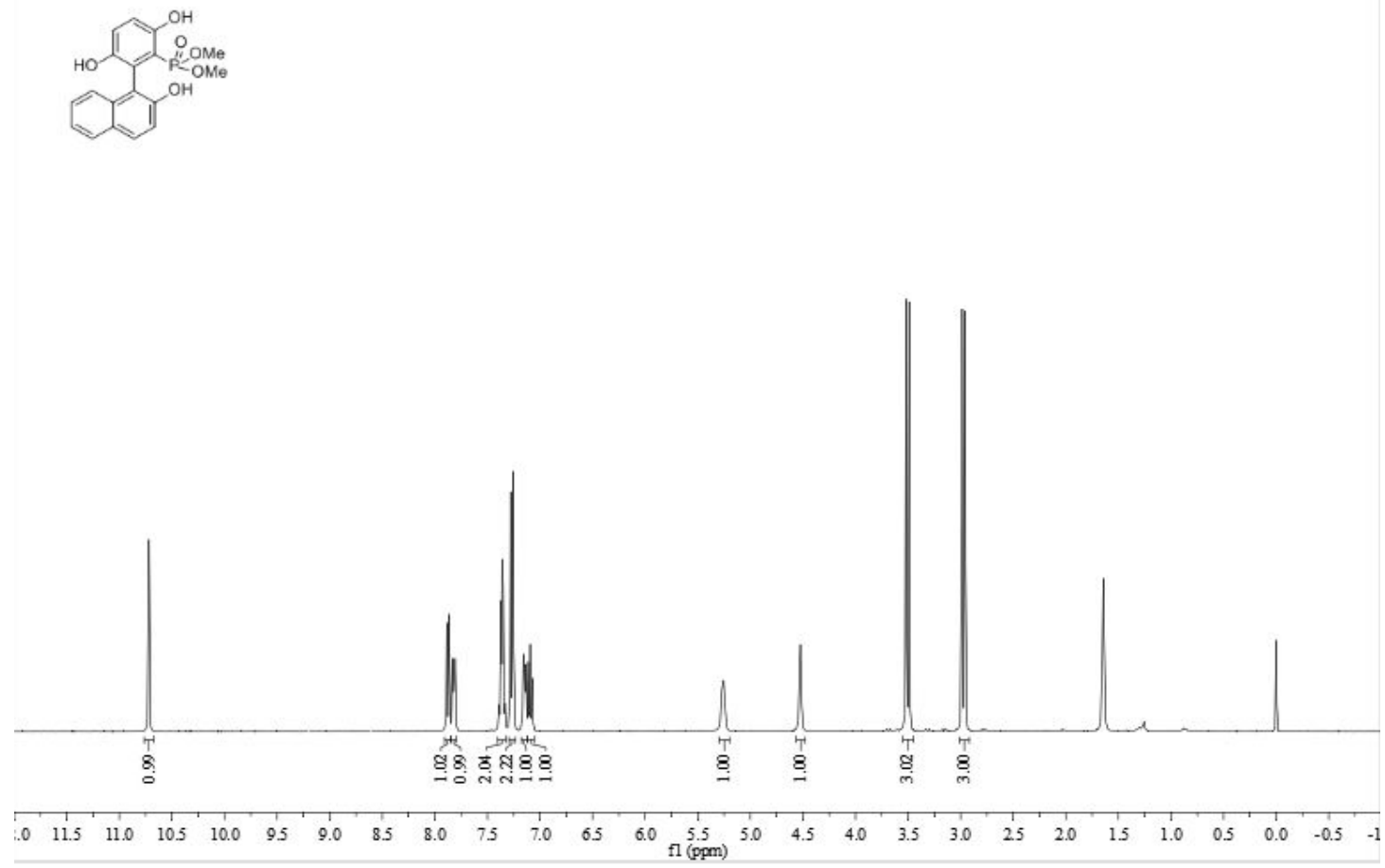
${ }^{31} \mathrm{P}$ NMR for compound 4 ba $\left(162 \mathrm{MHz}, \mathrm{CDCl}_{3}\right.$ )

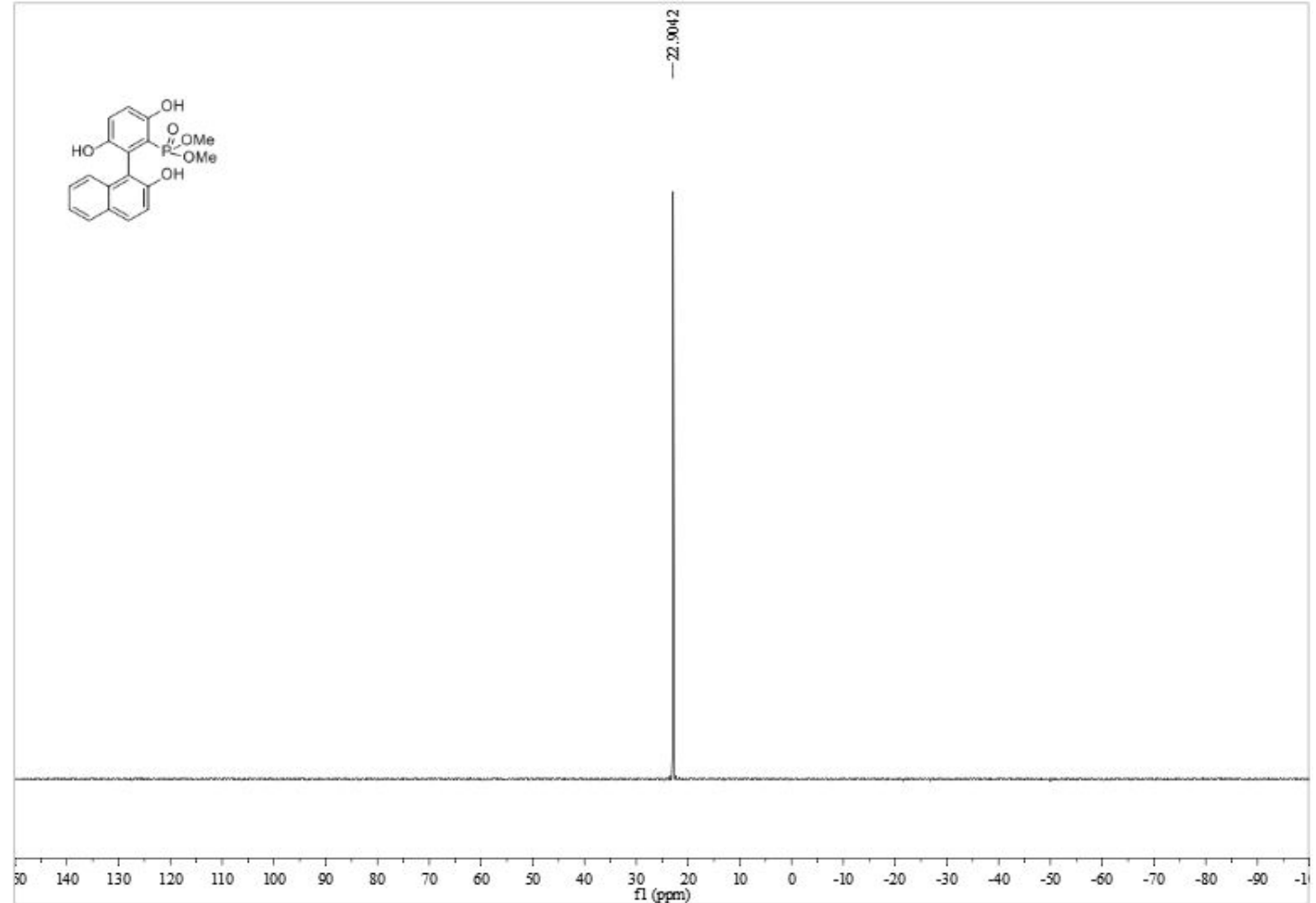

${ }^{13} \mathrm{C}\left\{{ }^{1} \mathrm{H}\right\} \mathrm{NMR}$ for compound $4 \mathbf{b a}\left(101 \mathrm{MHz}, \mathrm{CDCl}_{3}\right)$

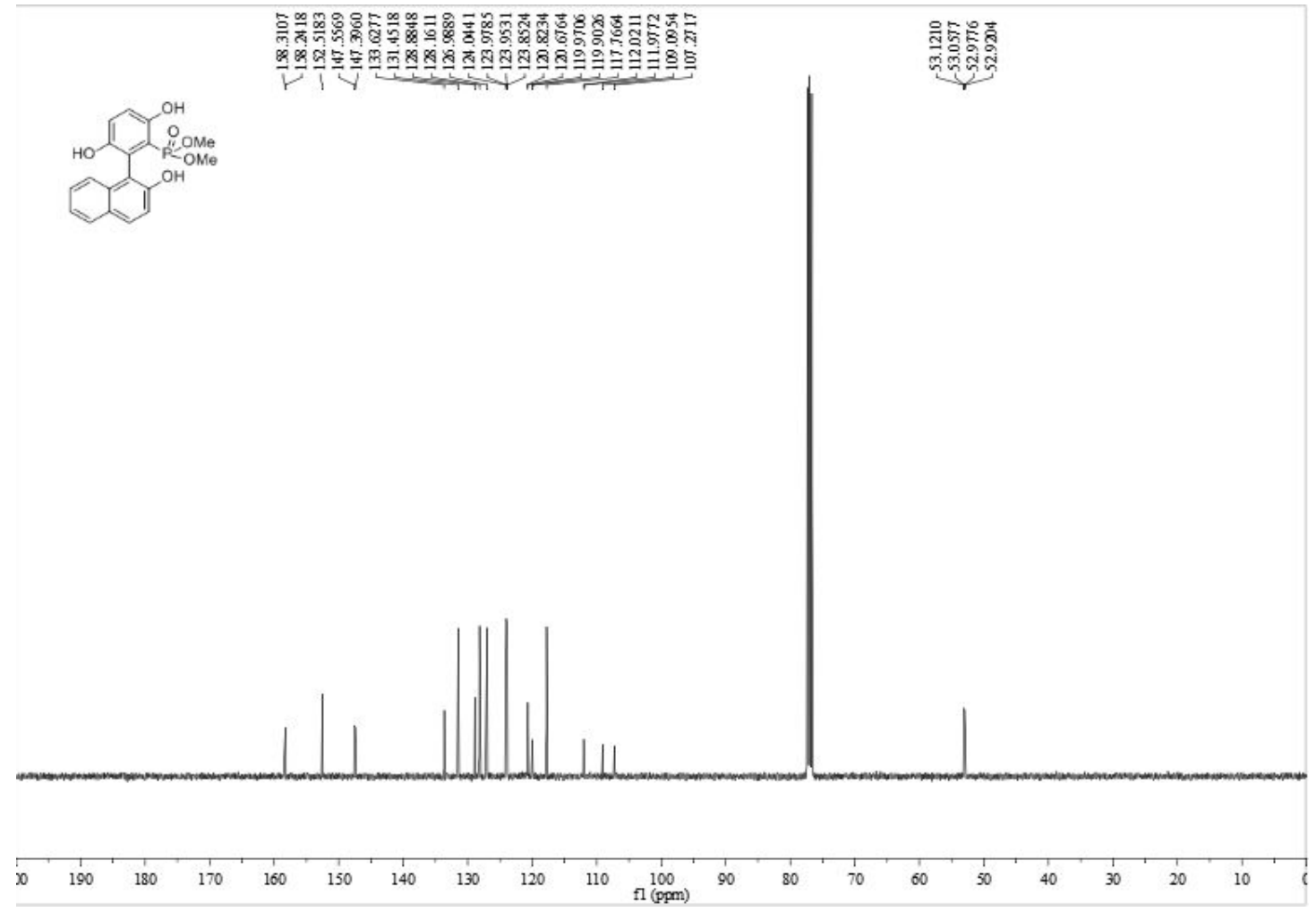


${ }^{1} \mathrm{H}$ NMR for compound $4 \mathbf{c a}\left(400 \mathrm{MHz}, \mathrm{CDCl}_{3}\right.$ )

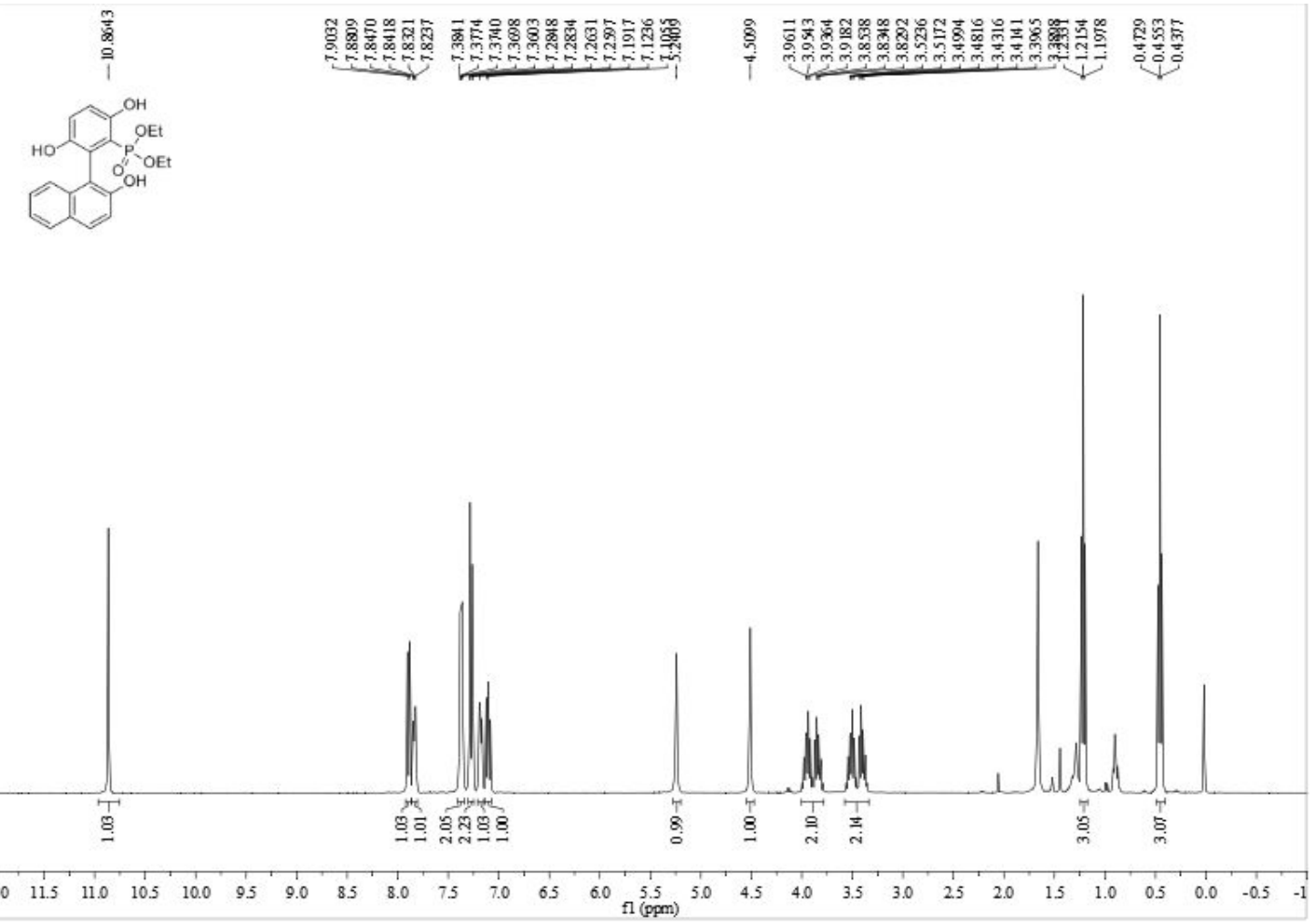

${ }^{31} \mathrm{P}$ NMR for compound $4 \mathrm{ca}\left(162 \mathrm{MHz}, \mathrm{CDCl}_{3}\right)$

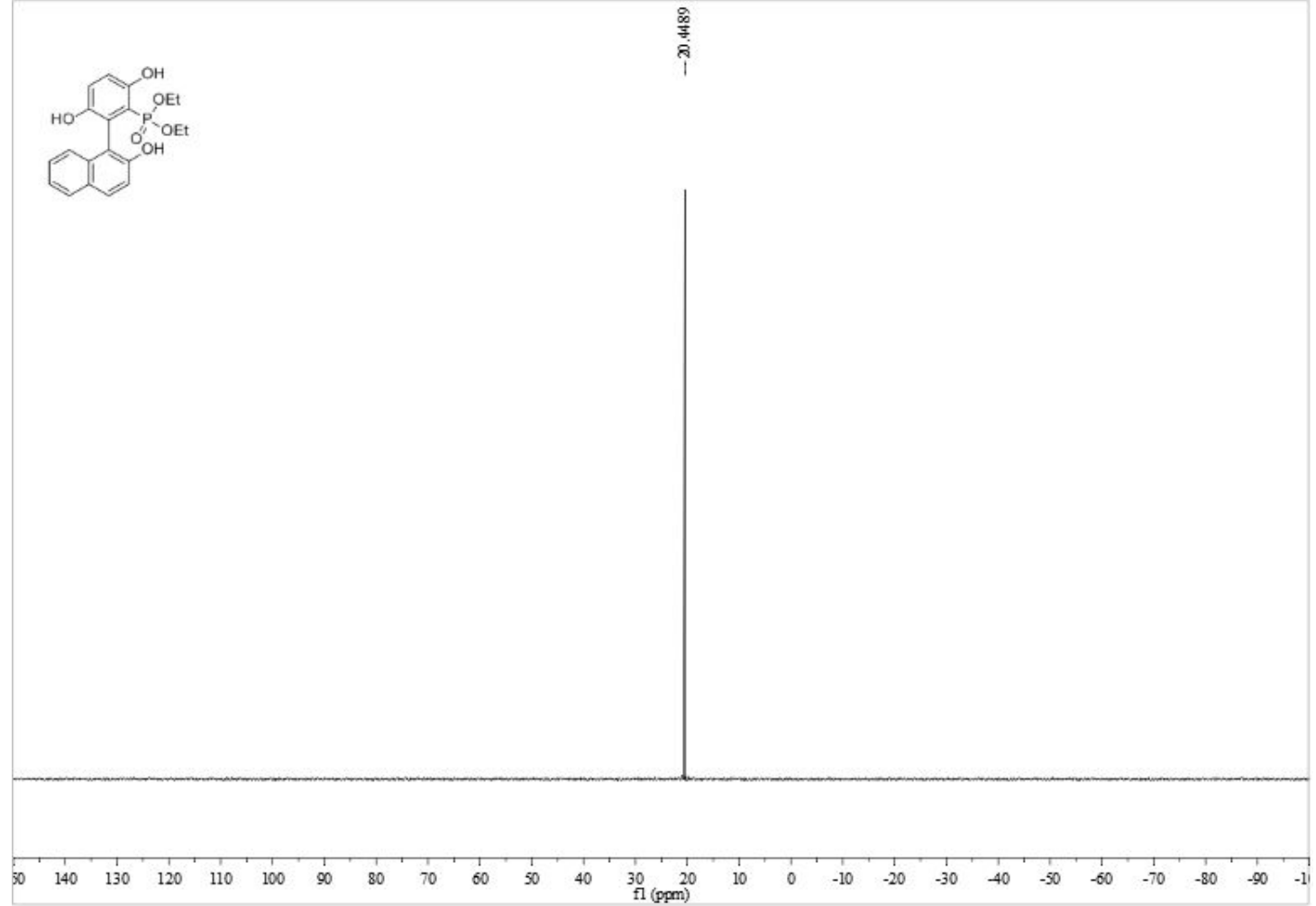


${ }^{13} \mathrm{C}\left\{{ }^{1} \mathrm{H}\right\}$ NMR for compound $4 \mathbf{c a}\left(101 \mathrm{MHz}, \mathrm{CDCl}_{3}\right)$

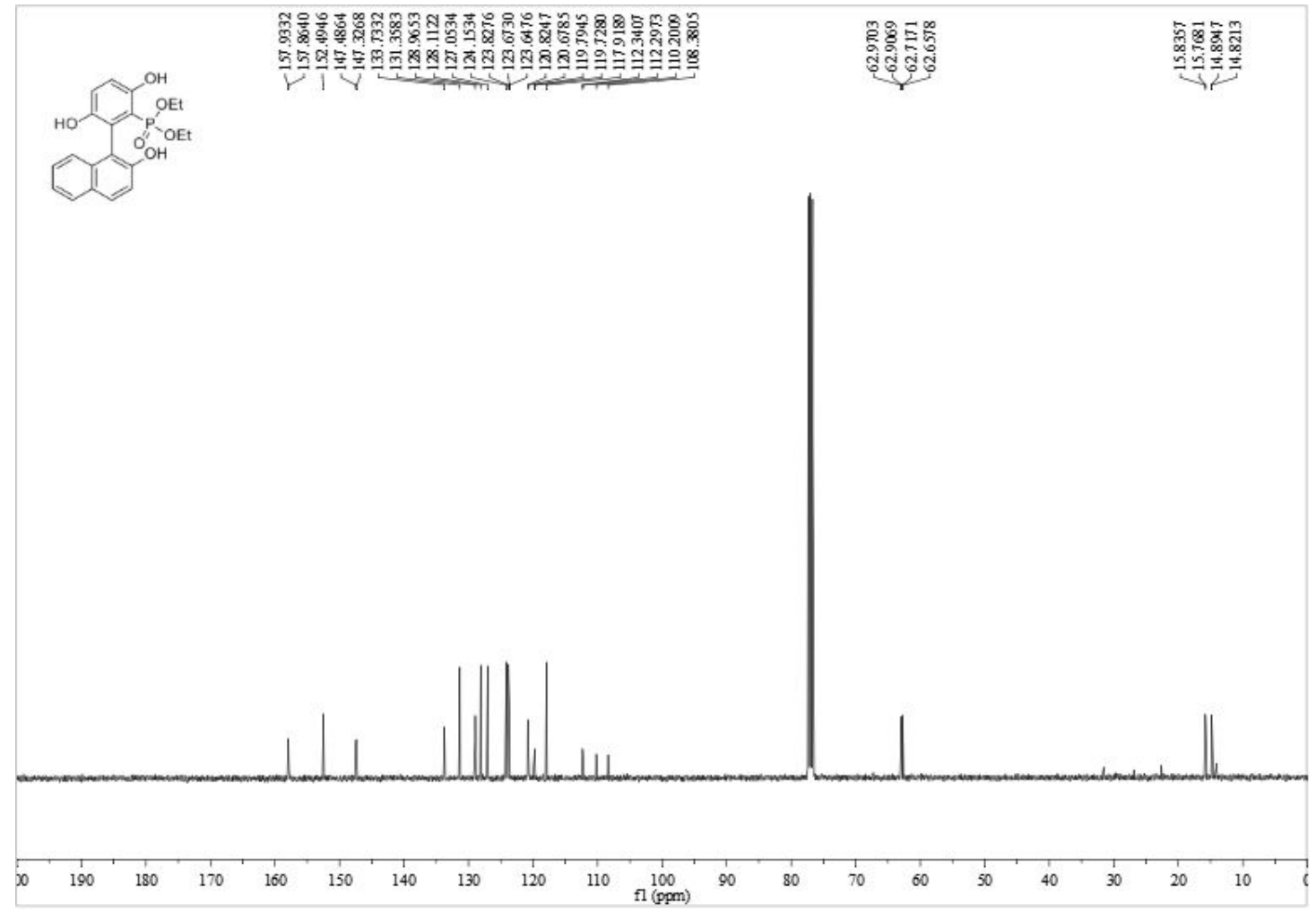

${ }^{1} \mathrm{H}$ NMR for compound $4 \mathbf{d a}\left(400 \mathrm{MHz}, \mathrm{CDCl}_{3}\right.$ )

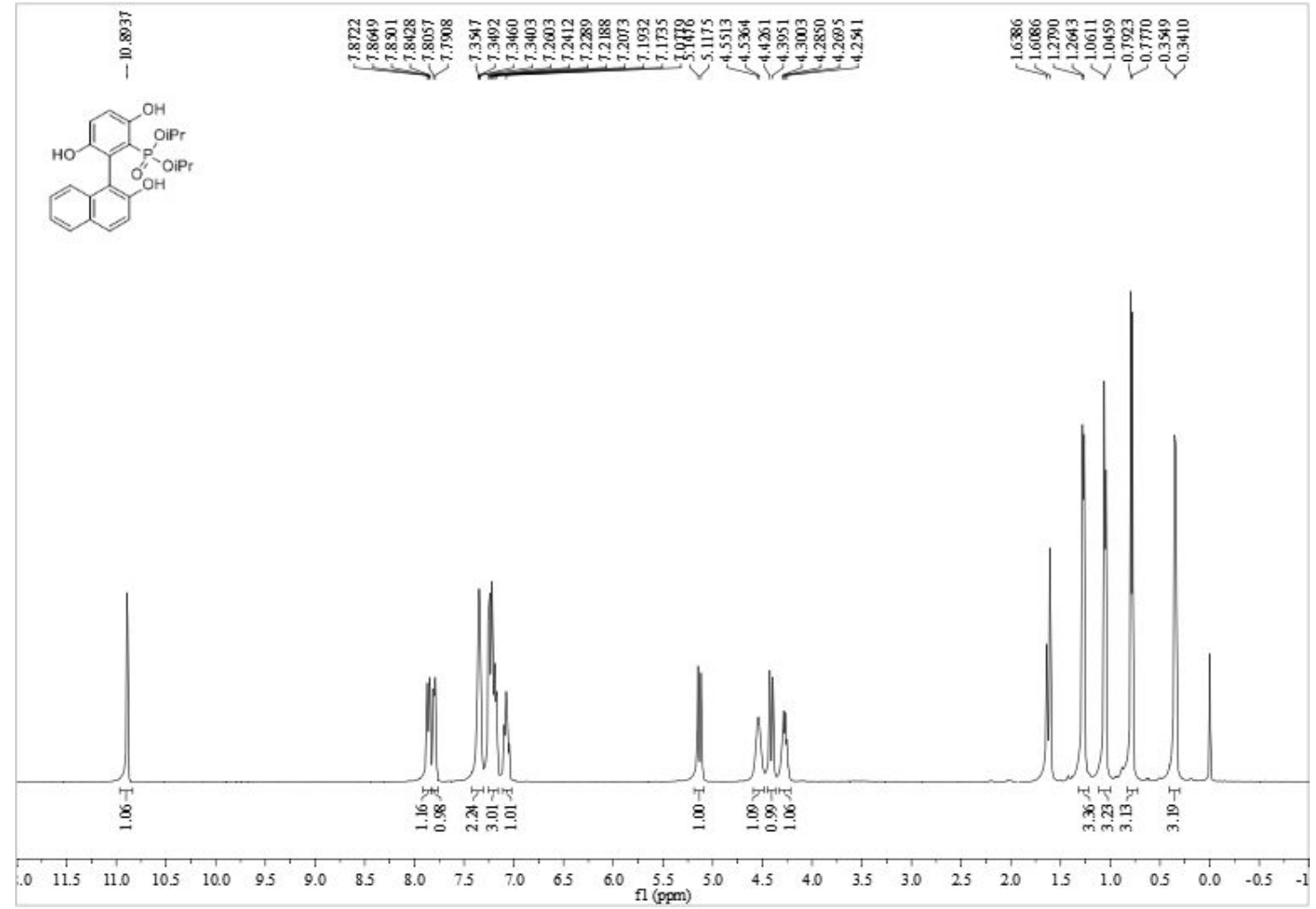


${ }^{31} \mathrm{P}$ NMR for compound 4da $\left(162 \mathrm{MHz}, \mathrm{CDCl}_{3}\right)$

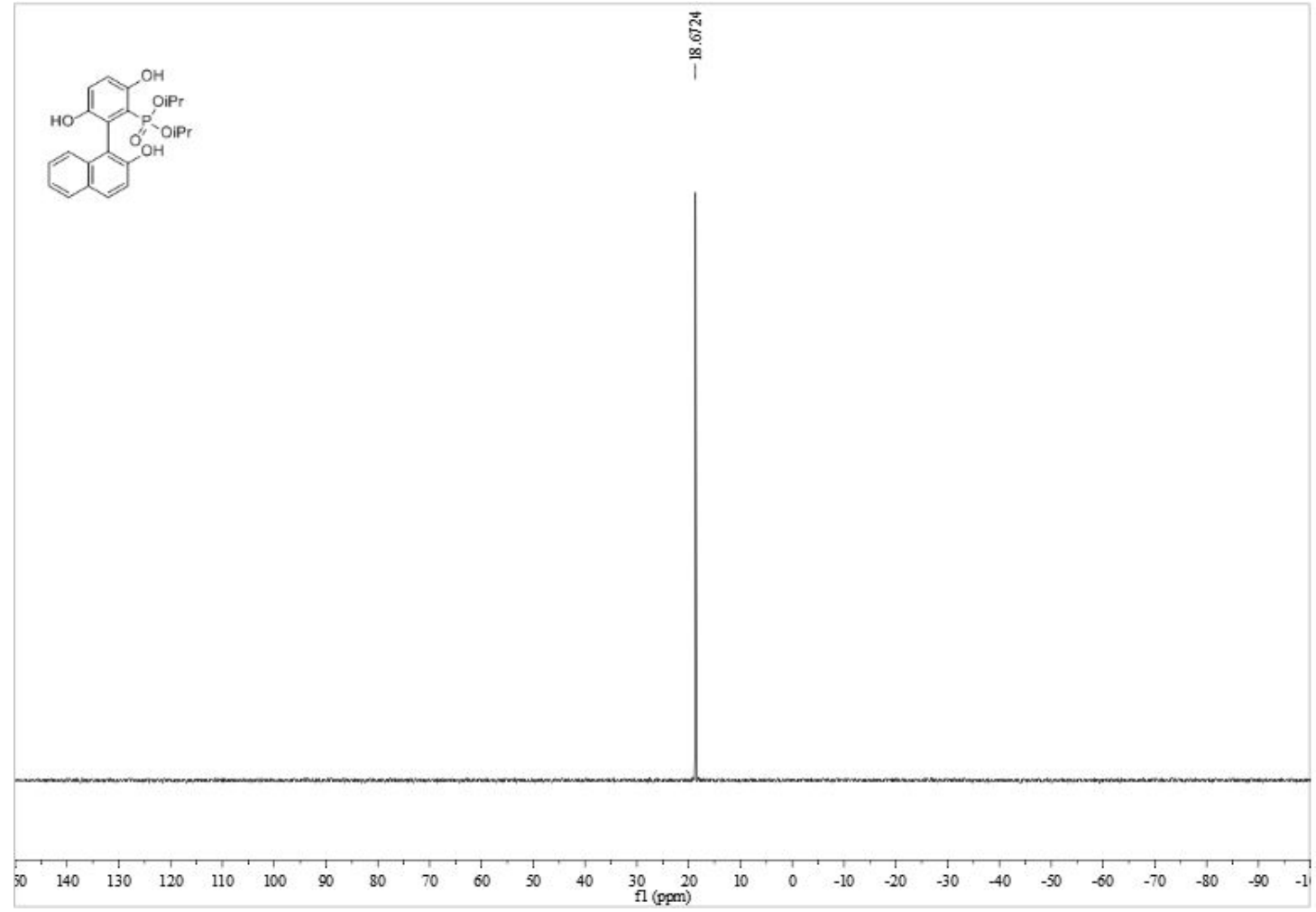

${ }^{13} \mathrm{C}\left\{{ }^{1} \mathrm{H}\right\}$ NMR for compound $4 \mathbf{d a}\left(101 \mathrm{MHz}, \mathrm{CDCl}_{3}\right)$

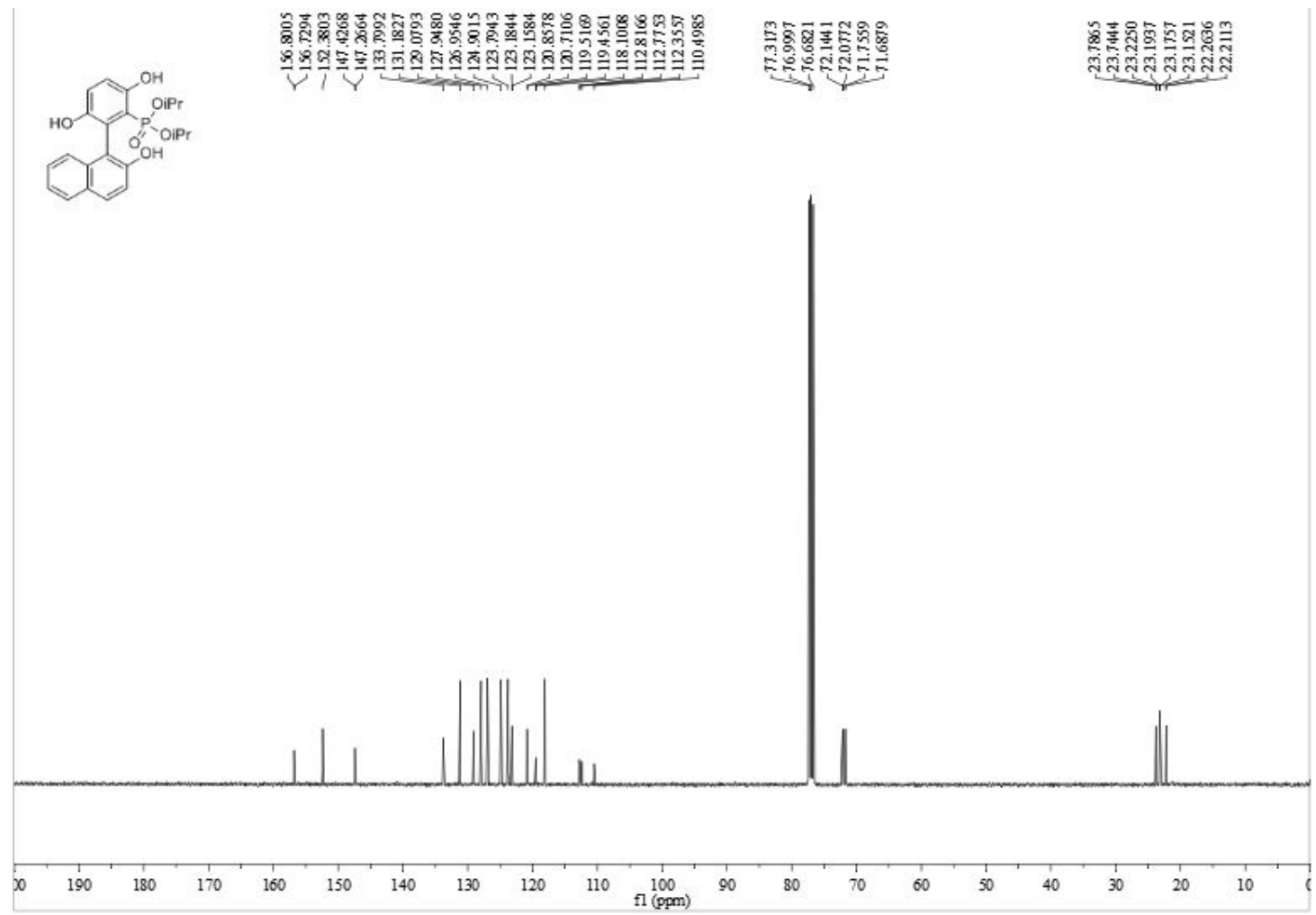


${ }^{1} \mathrm{H}$ NMR for compound 4 ea $\left(400 \mathrm{MHz}, \mathrm{CDCl}_{3}\right.$ )

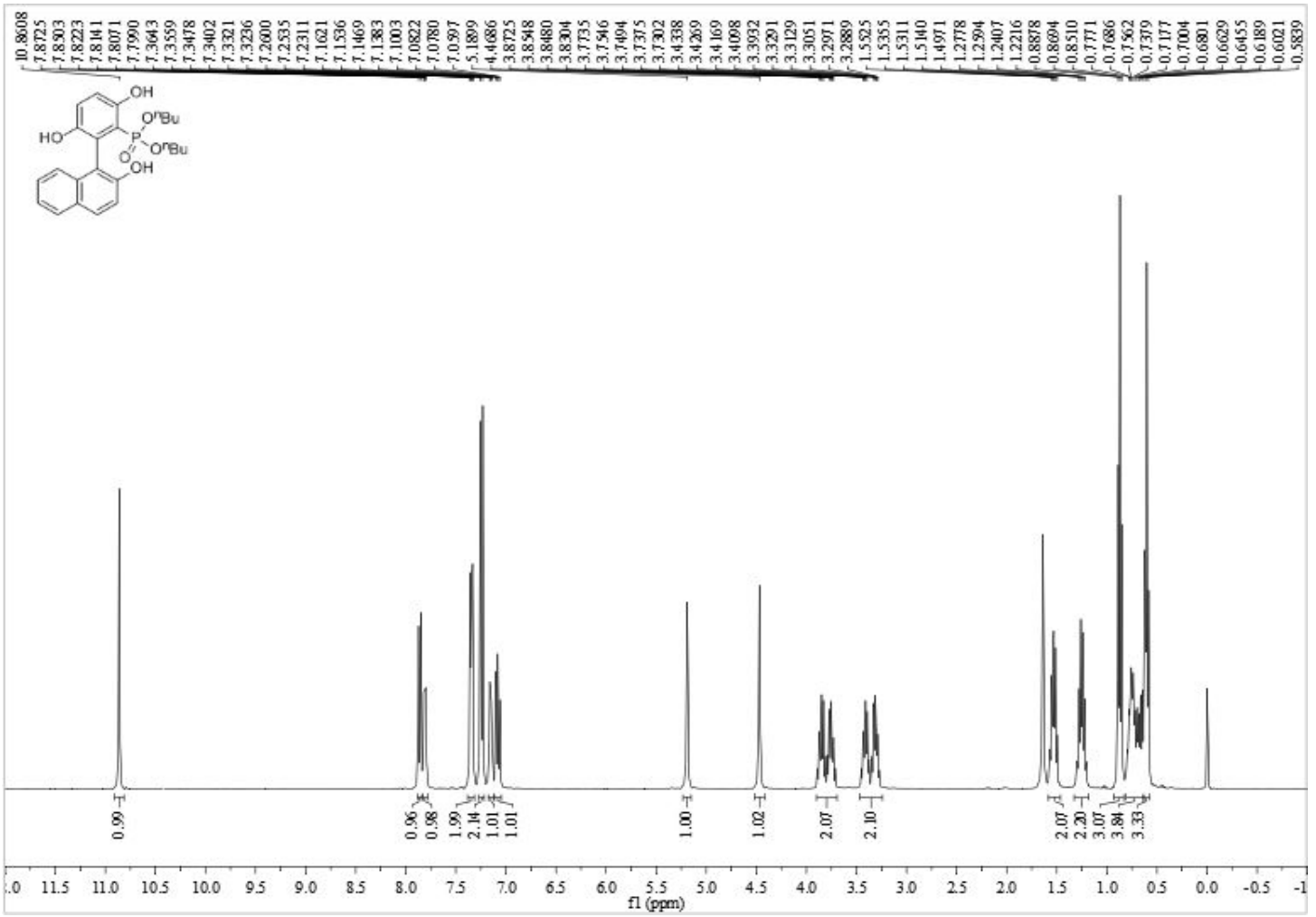

${ }^{31} \mathrm{P}$ NMR for compound 4 ea $\left(162 \mathrm{MHz}, \mathrm{CDCl}_{3}\right)$

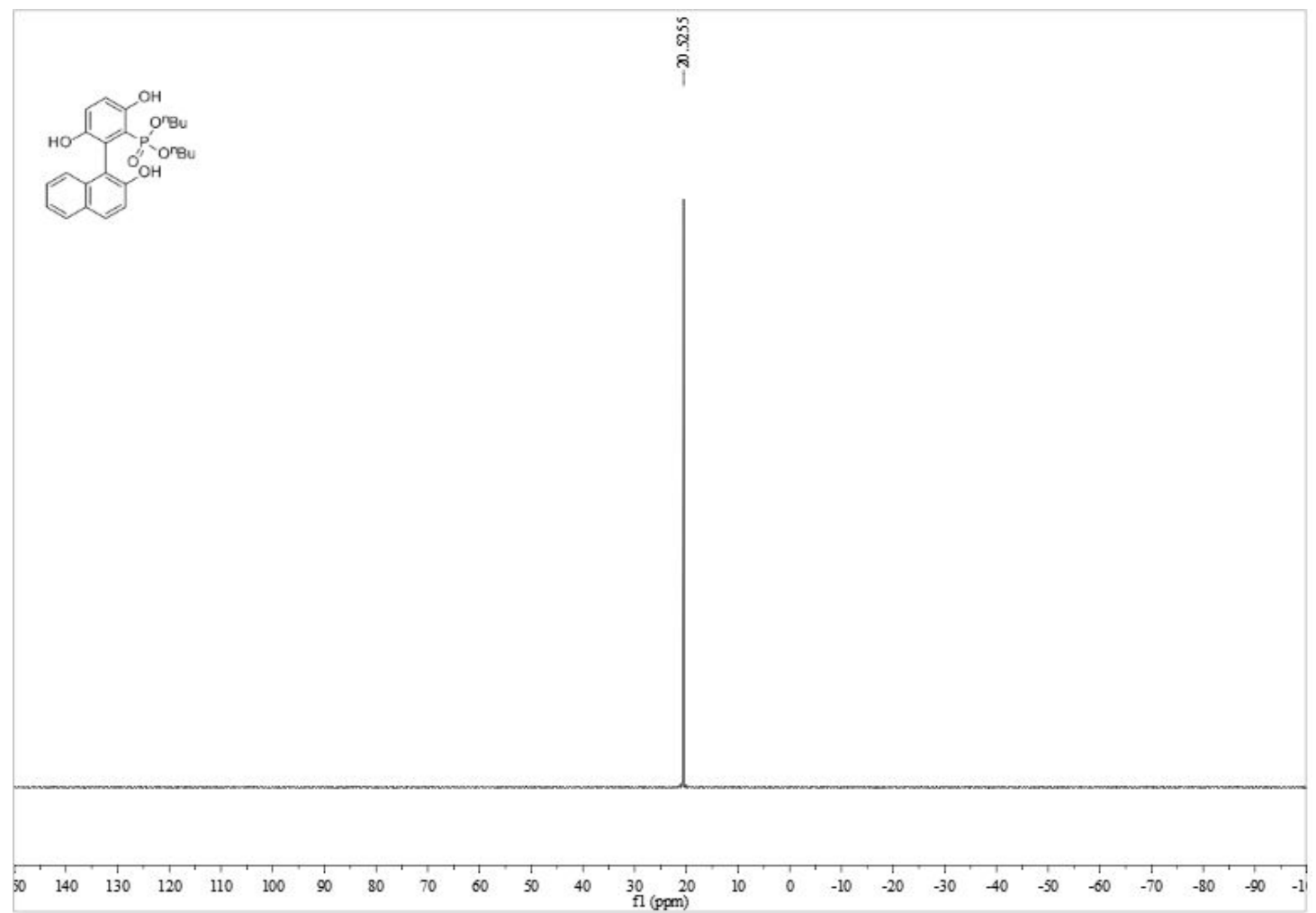


${ }^{13} \mathrm{C}\left\{{ }^{1} \mathrm{H}\right\}$ NMR for compound 4ea $\left(101 \mathrm{MHz}, \mathrm{CDCl}_{3}\right)$

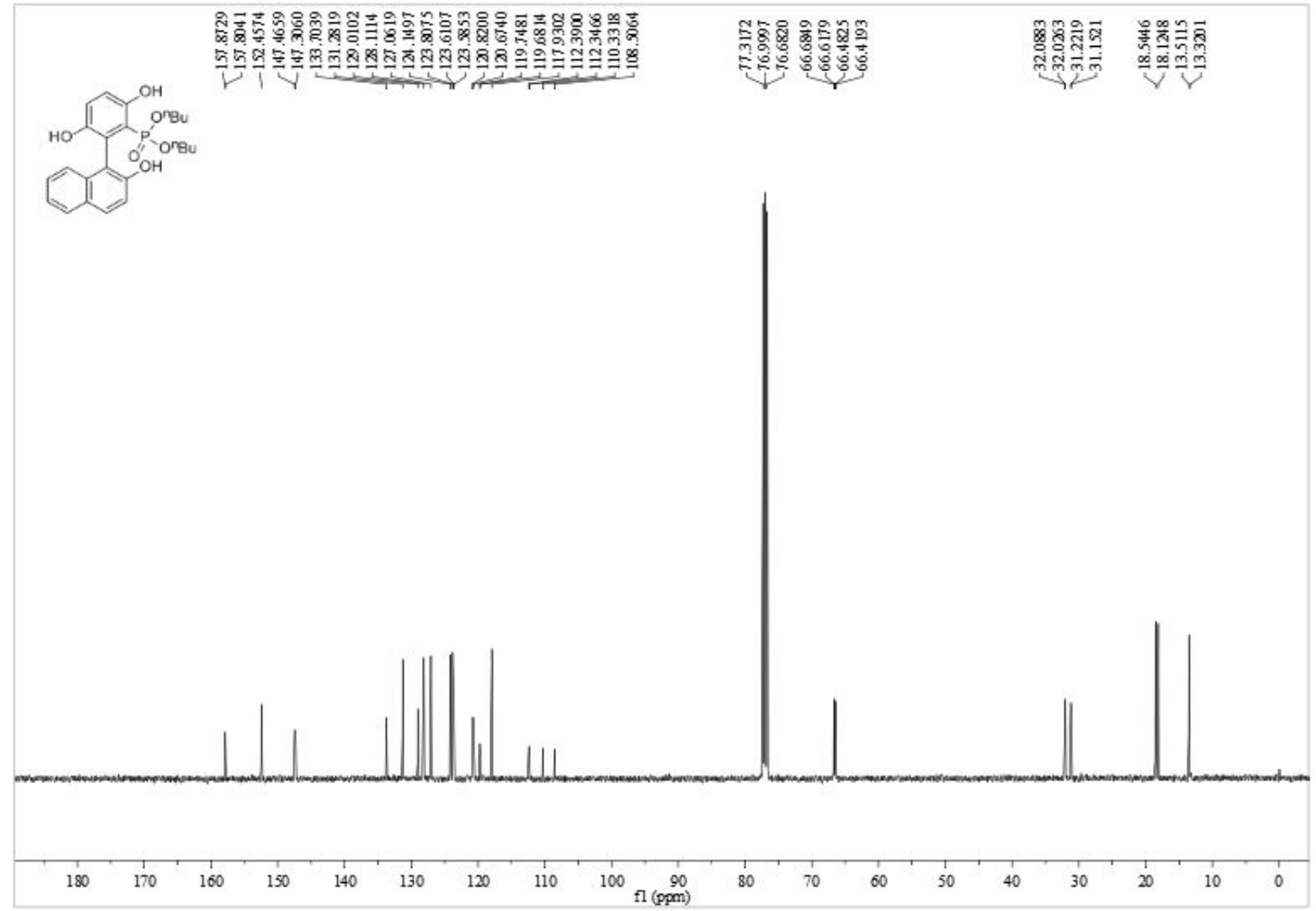

${ }^{1} \mathrm{H}$ NMR for compound 4 fa $\left(400 \mathrm{MHz}, \mathrm{CDCl}_{3}\right)$

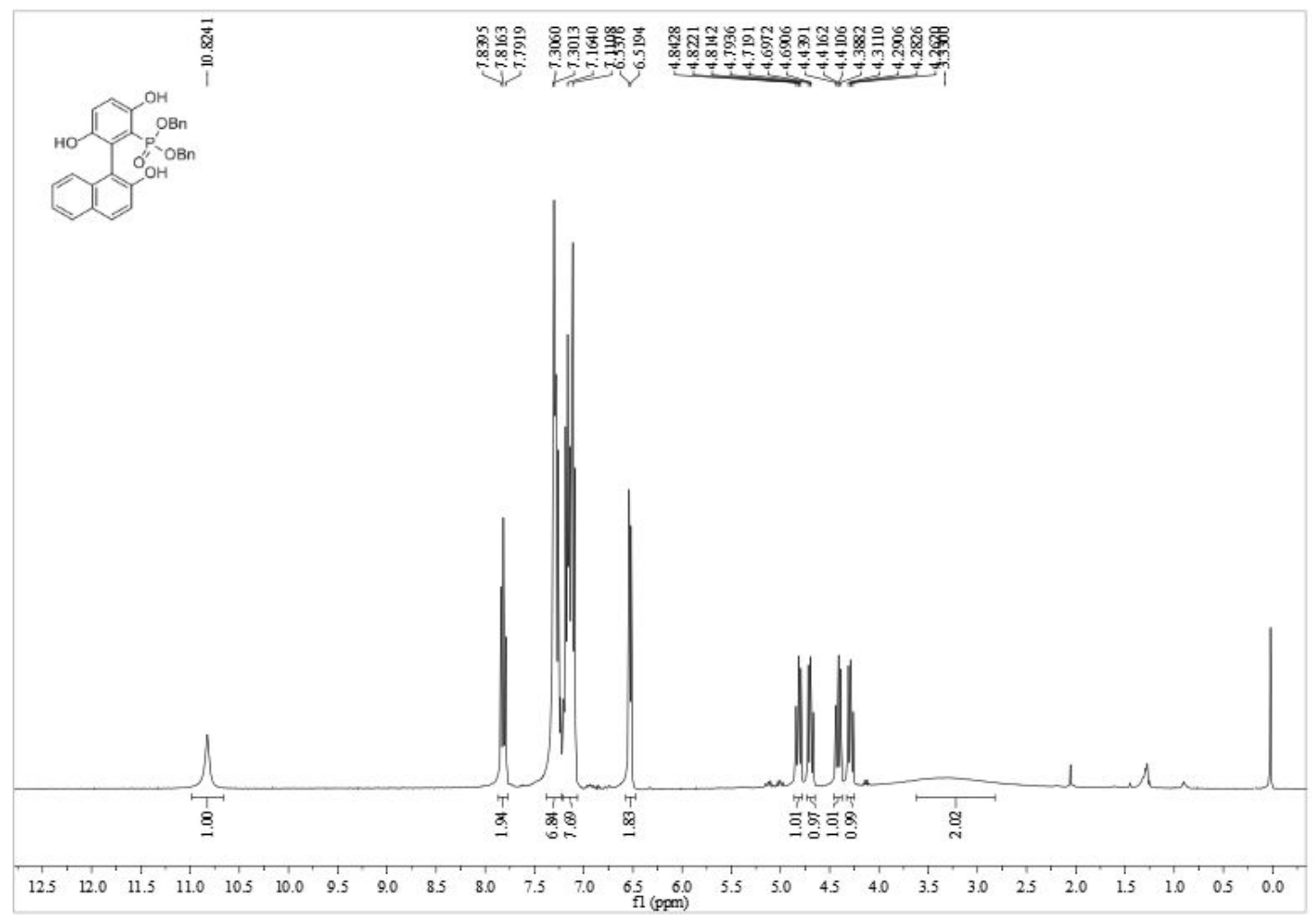


${ }^{31} \mathrm{P}$ NMR for compound 4 fa $\left(162 \mathrm{MHz}, \mathrm{CDCl}_{3}\right)$

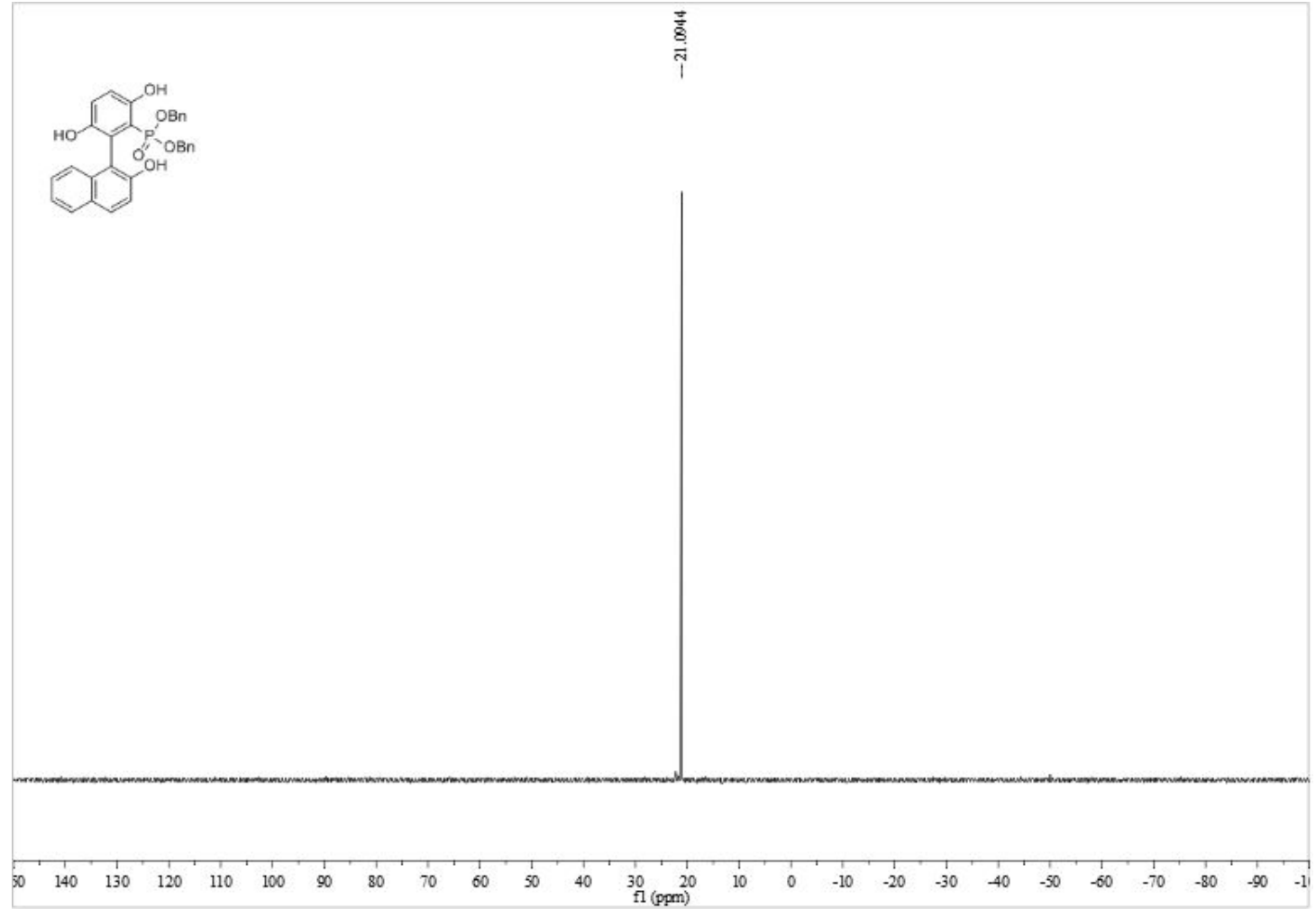

${ }^{13} \mathrm{C}\left\{{ }^{1} \mathrm{H}\right\} \mathrm{NMR}$ for compound 4 fa $\left(101 \mathrm{MHz}, \mathrm{CDCl}_{3}\right)$

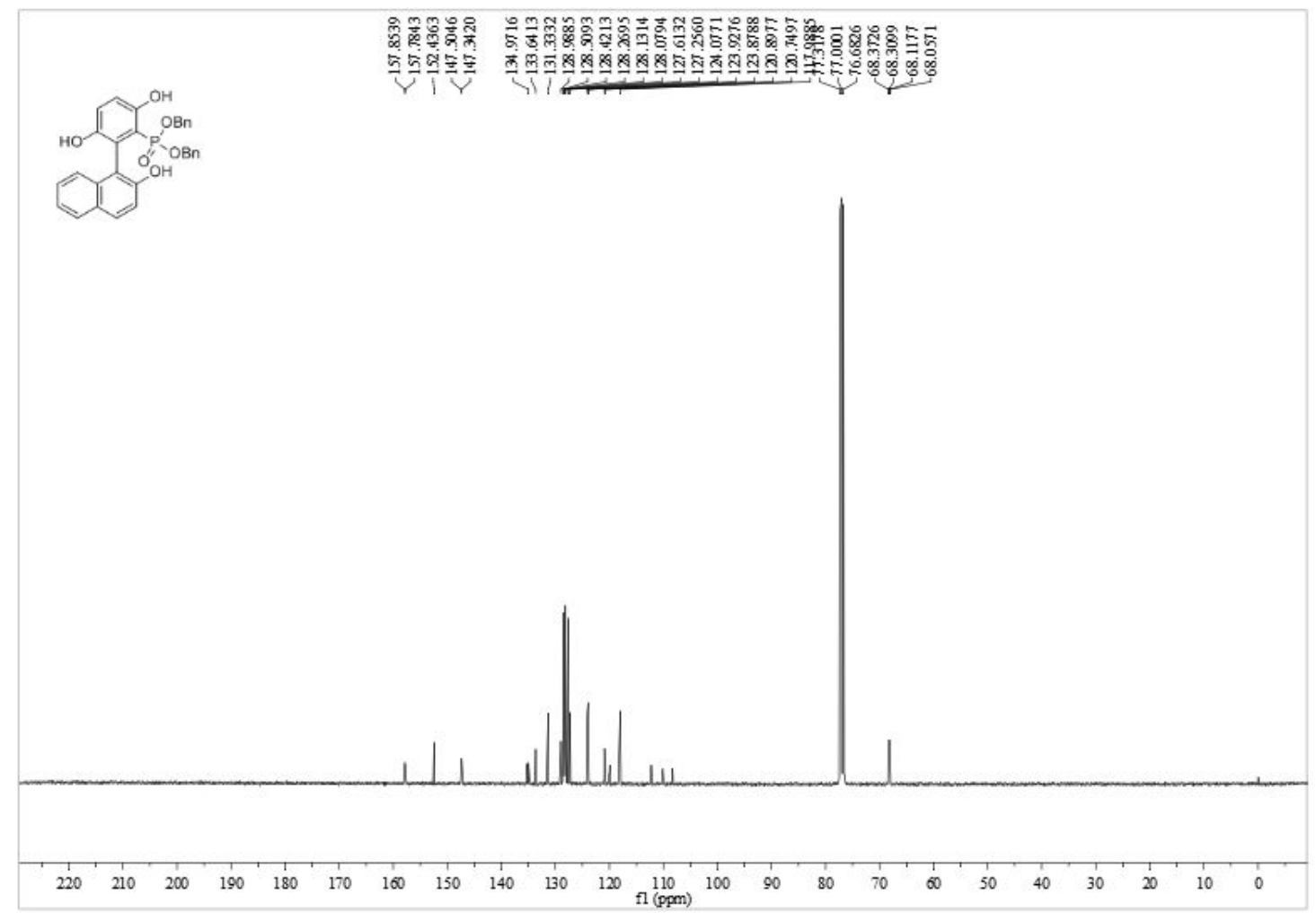


${ }^{1} \mathrm{H}$ NMR for compound 4cb (400 MHz, DMSO-d 6 )
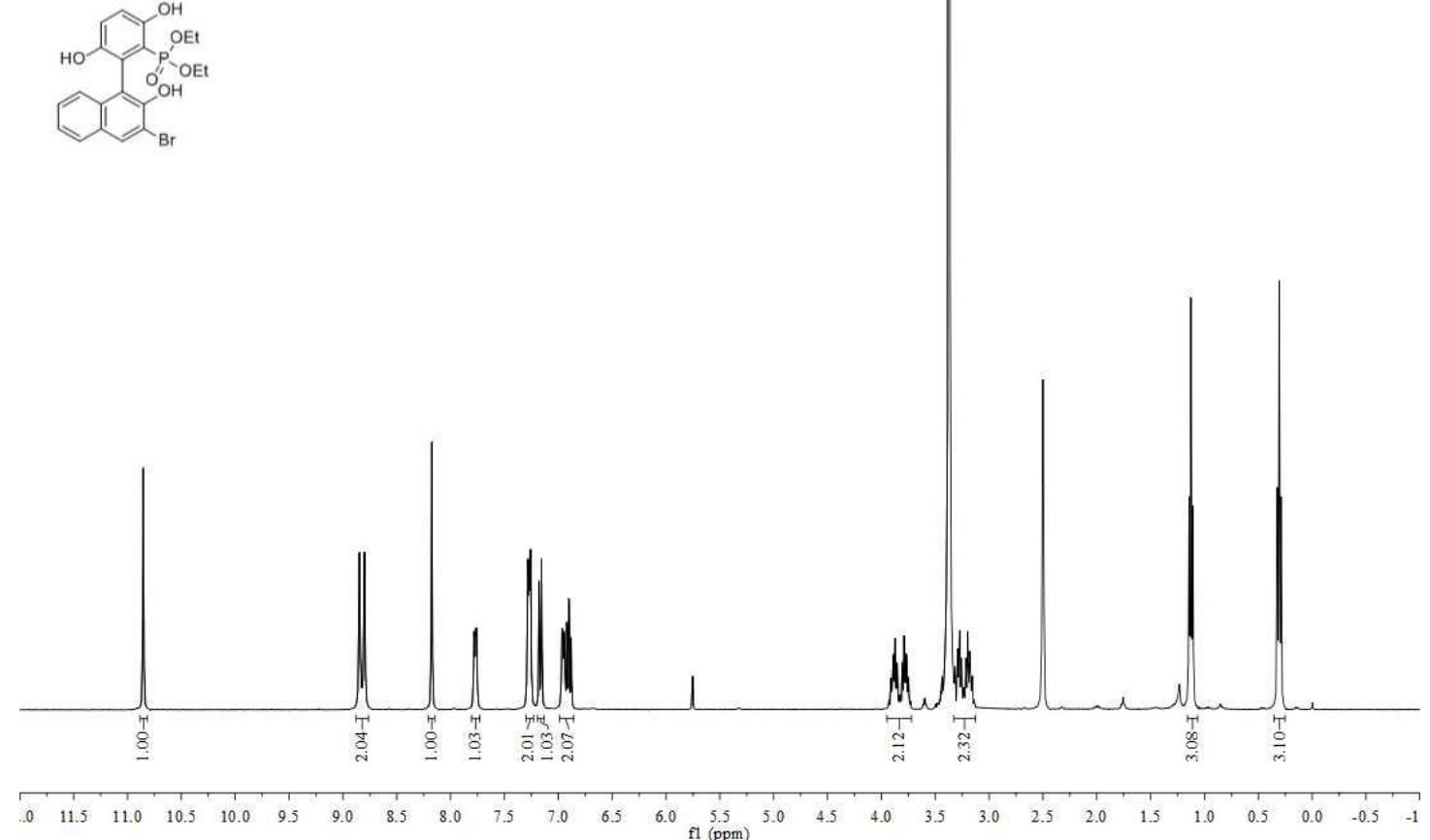

${ }^{31} \mathrm{P}$ NMR for compound 4cb (162 MHz, DMSO- $\mathrm{d}_{6}$ )

蒂

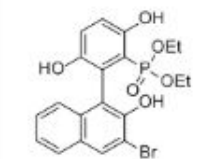

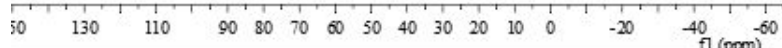


${ }^{13} \mathrm{C}\left\{{ }^{1} \mathrm{H}\right\}$ NMR for compound 4cb $\left(101 \mathrm{MHz}\right.$, DMSO-d $\left.{ }_{6}\right)$
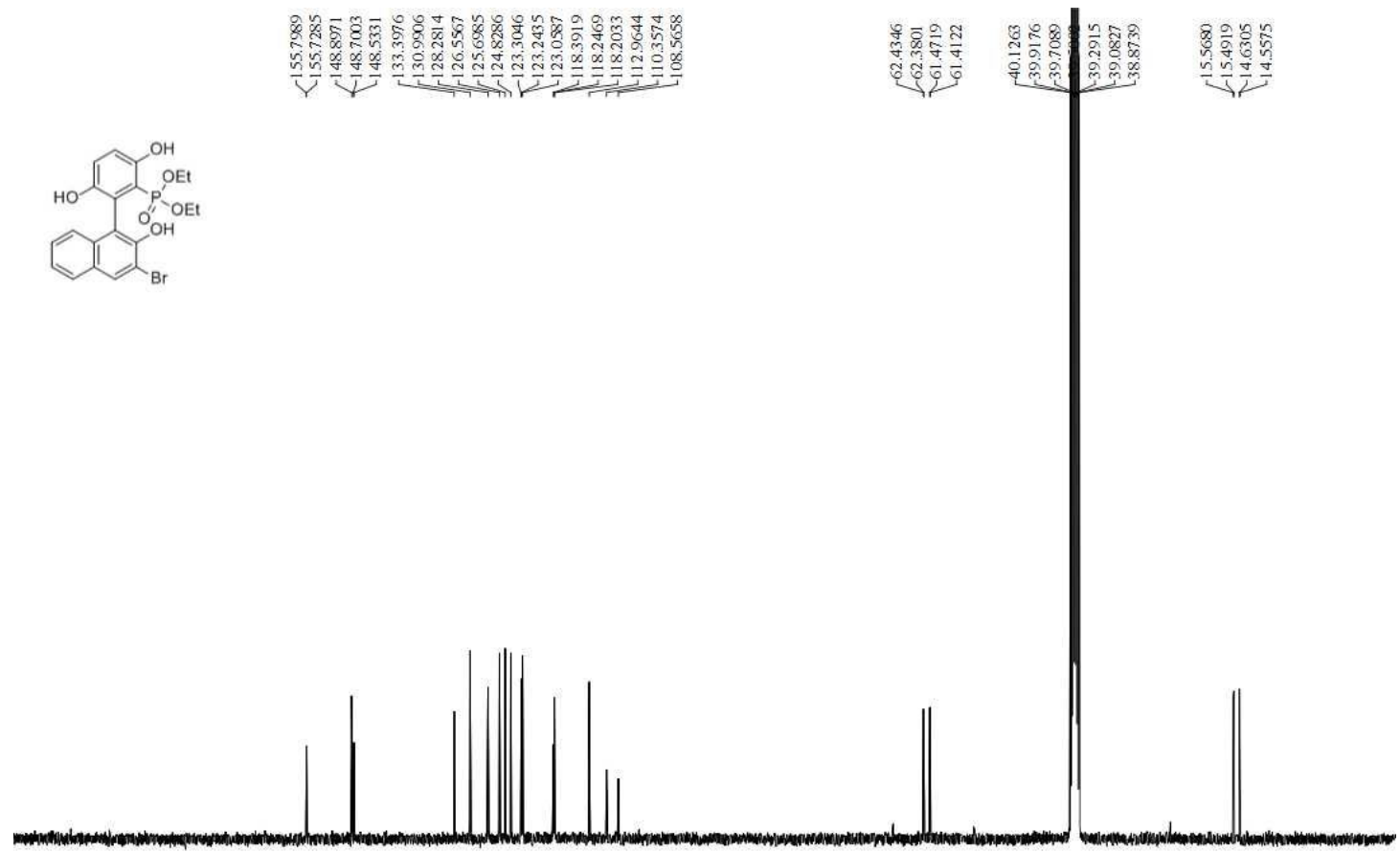

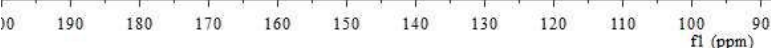

${ }^{1} \mathrm{H}$ NMR for compound 4cc (400 MHz, DMSO-d ${ }_{6}$ )

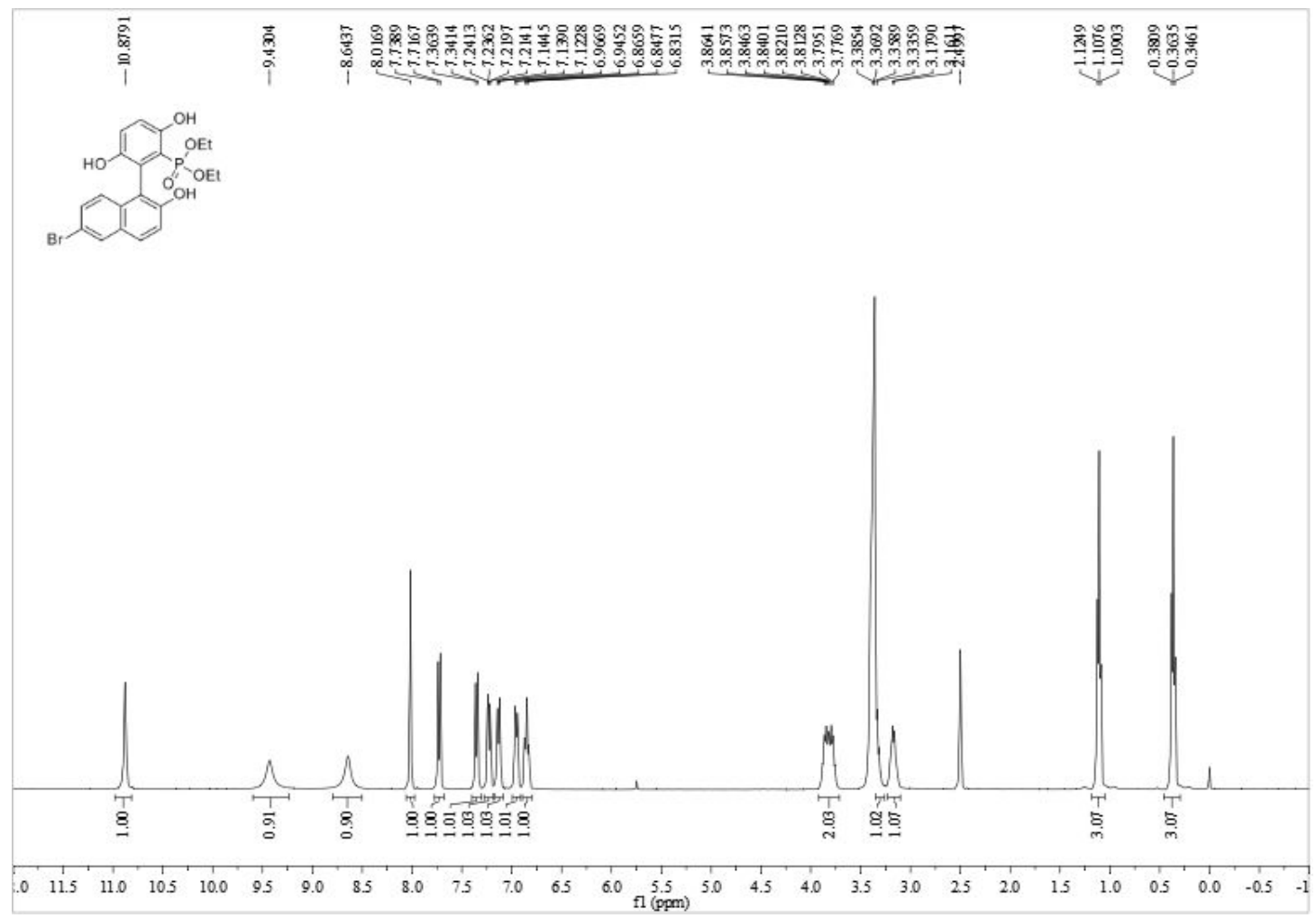


${ }^{31} \mathrm{P}$ NMR for compound 4ce (162 MHz, DMSO- $\left.\mathrm{d}_{6}\right)$

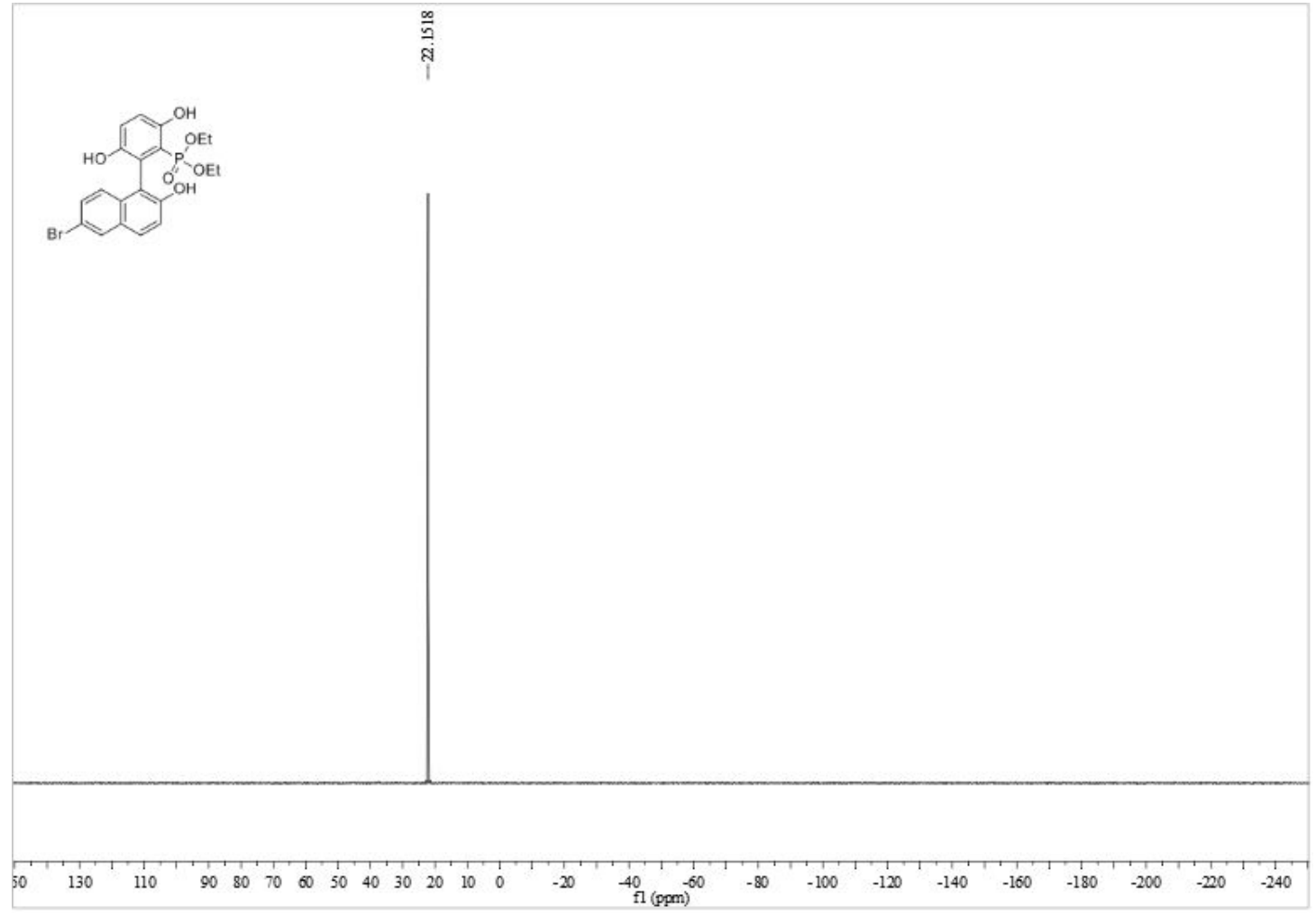

${ }^{13} \mathrm{C}\left\{{ }^{1} \mathrm{H}\right\}$ NMR for compound 4cc (151 MHz, DMSO-d $\left.\mathrm{d}_{6}\right)$

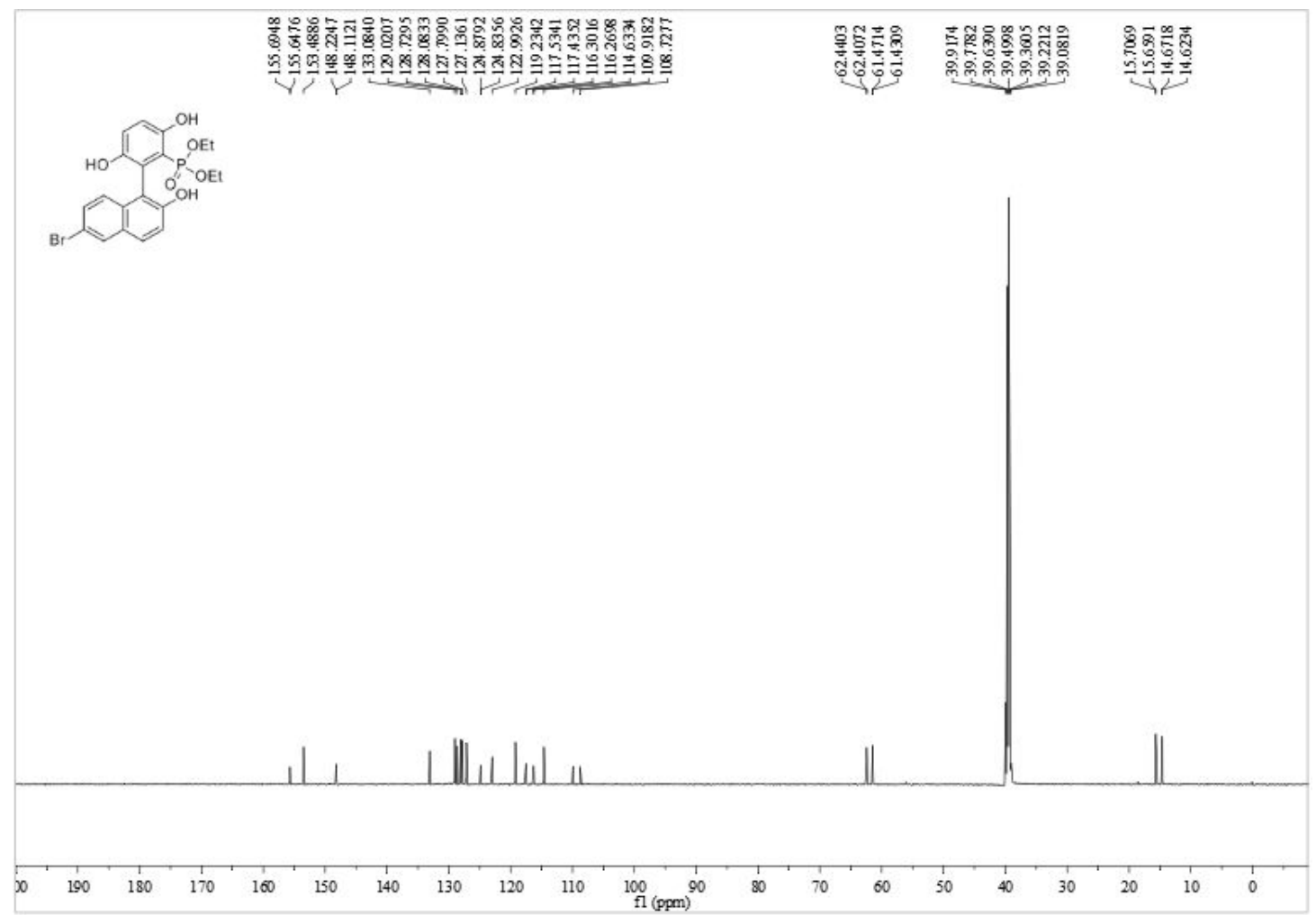


${ }^{1} \mathrm{H}$ NMR for compound $\mathbf{4} \mathbf{c d}\left(400 \mathrm{MHz}, \mathrm{DMSO}-\mathrm{d}_{6}\right)$

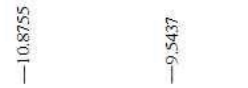

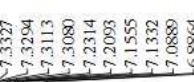

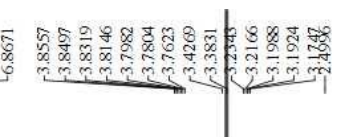

ஜ요유.
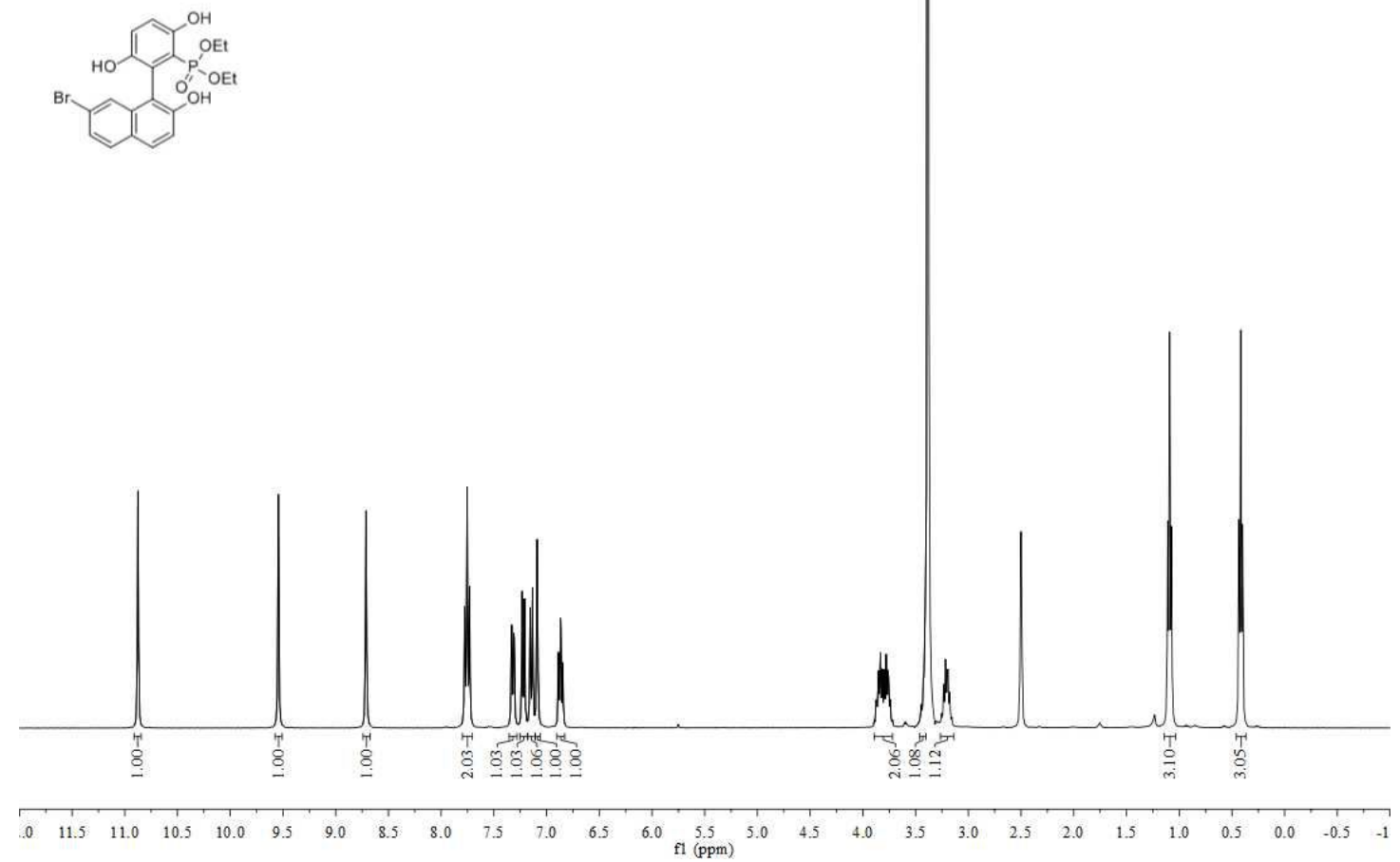

${ }^{31} \mathrm{P}$ NMR for compound 4cd (162 MHz, DMSO- $\left.\mathrm{d}_{6}\right)$

옹

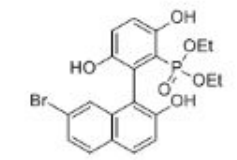

$\begin{array}{lllllllllllllllllllllllllllllllllllll}50 & 130 & 110 & 90 & 80 & 70 & 60 & 50 & 40 & 30 & 20 & 10 & 0 & -20 & -40 & -60 & -80 & -100 & -120 & -140 & -160 & -180 & -200 & -220 & -240\end{array}$ 
${ }^{13} \mathrm{C}\left\{{ }^{1} \mathrm{H}\right\}$ NMR for compound $4 \mathbf{c d}\left(101 \mathrm{MHz}\right.$, DMSO- $\mathrm{d}_{6}$ )

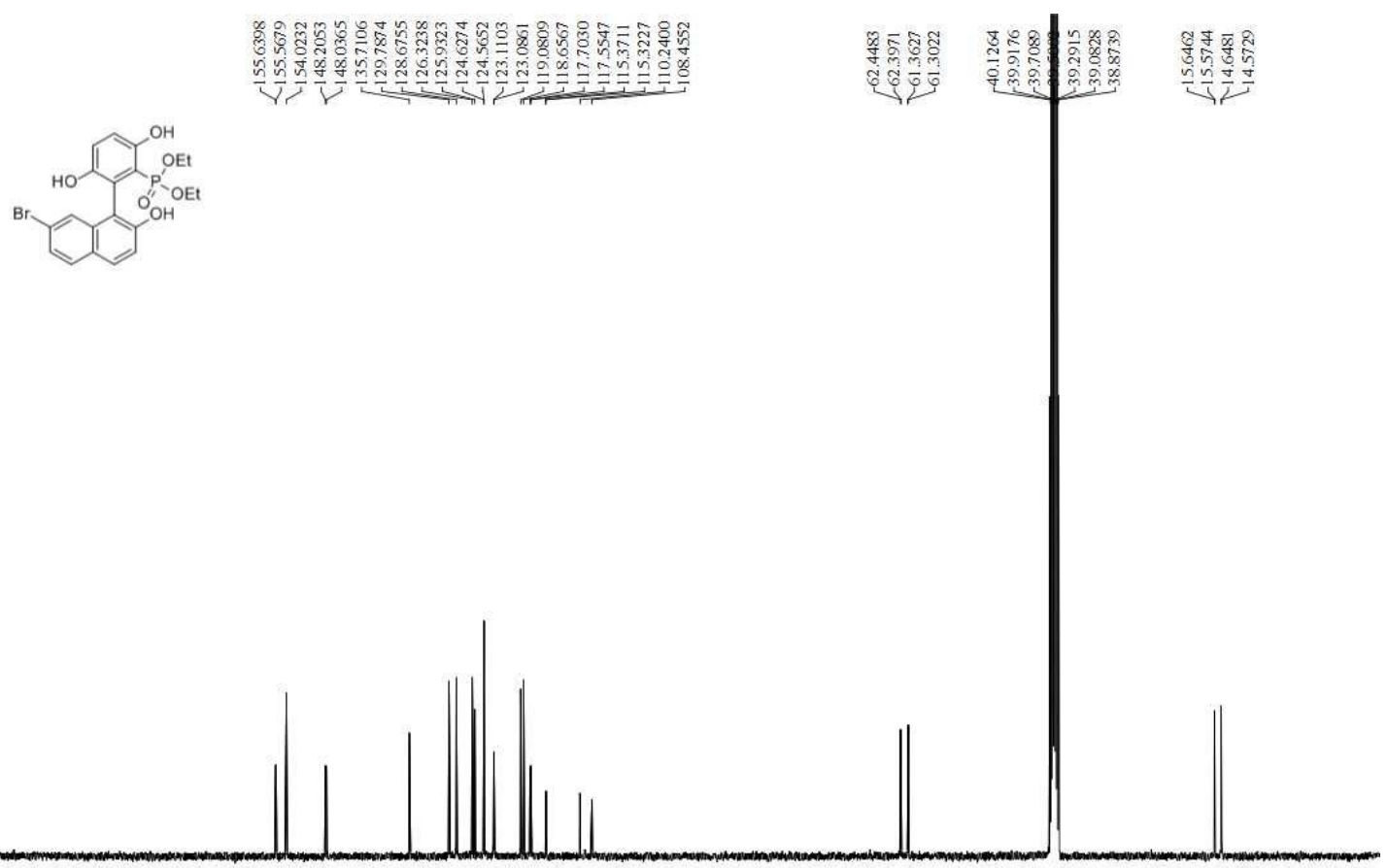

$\begin{array}{lllllllllllllllllllll}10 & 190 & 180 & 170 & 160 & 150 & 140 & 130 & 120 & 110 & 100 & 100 & 80 & 70 & 60 & 50 & 40 & 30 & 20 & 10 & 0\end{array}$

${ }^{1} \mathrm{H}$ NMR for compound $4 \mathbf{c e}\left(400 \mathrm{MHz}, \mathrm{CDCl}_{3}\right)$

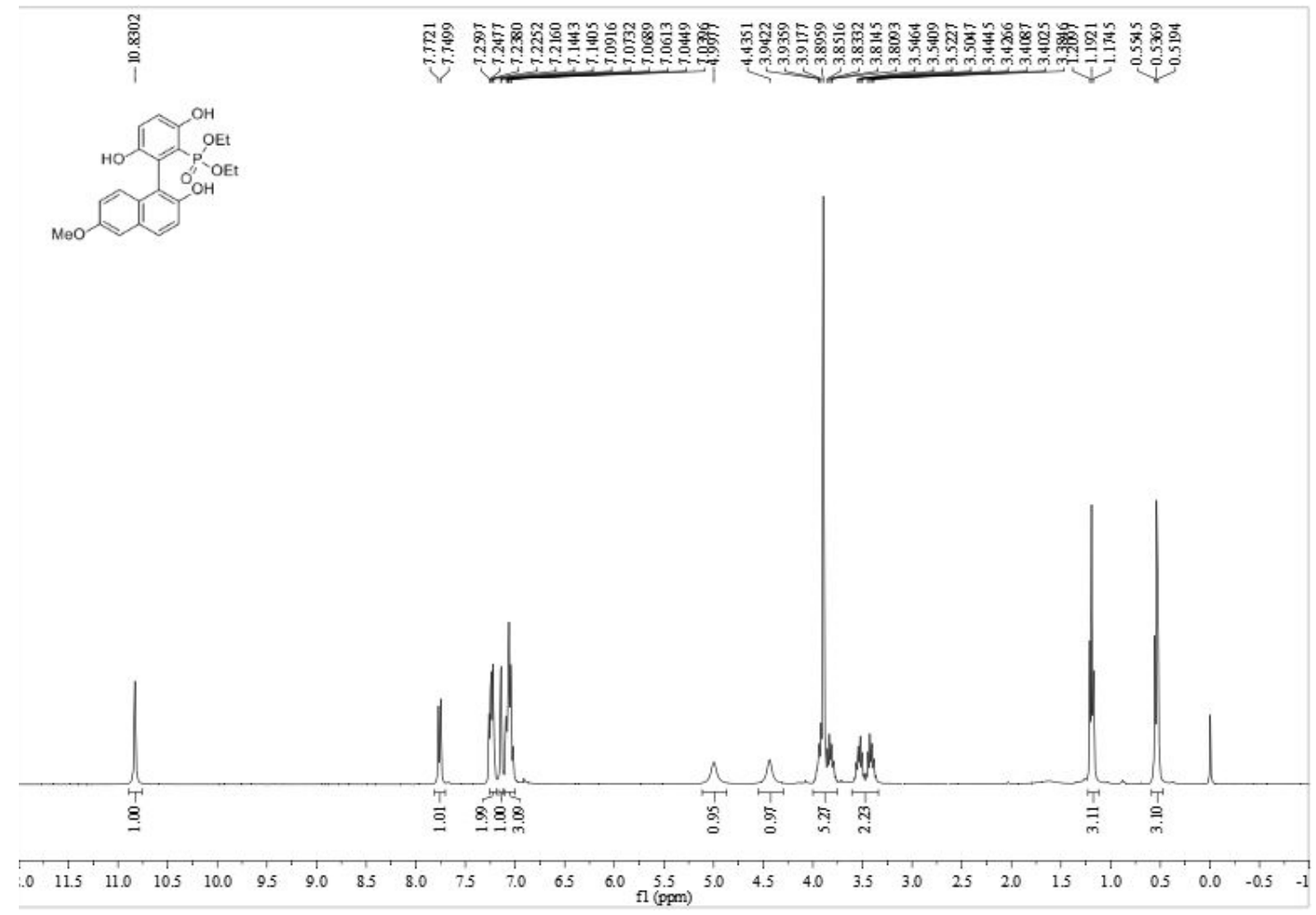


${ }^{31} \mathrm{P} \mathrm{NMR}$ for compound 4ce (162 $\left.\mathrm{MHz}, \mathrm{CDCl}_{3}\right)$

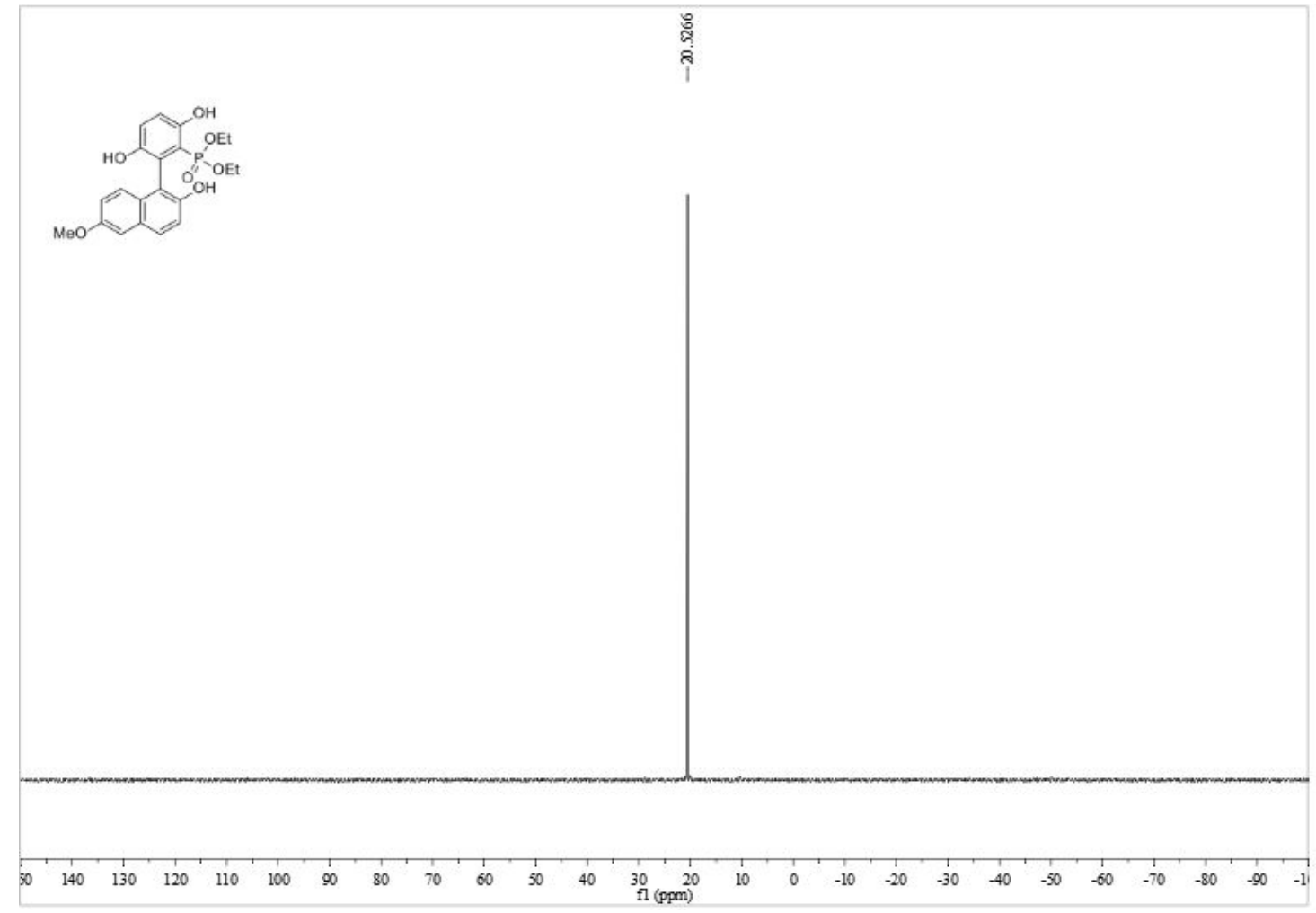

${ }^{13} \mathrm{C}\left\{{ }^{1} \mathrm{H}\right\}$ NMR for compound 4ce (101 $\left.\mathrm{MHz}, \mathrm{CDCl}_{3}\right)$

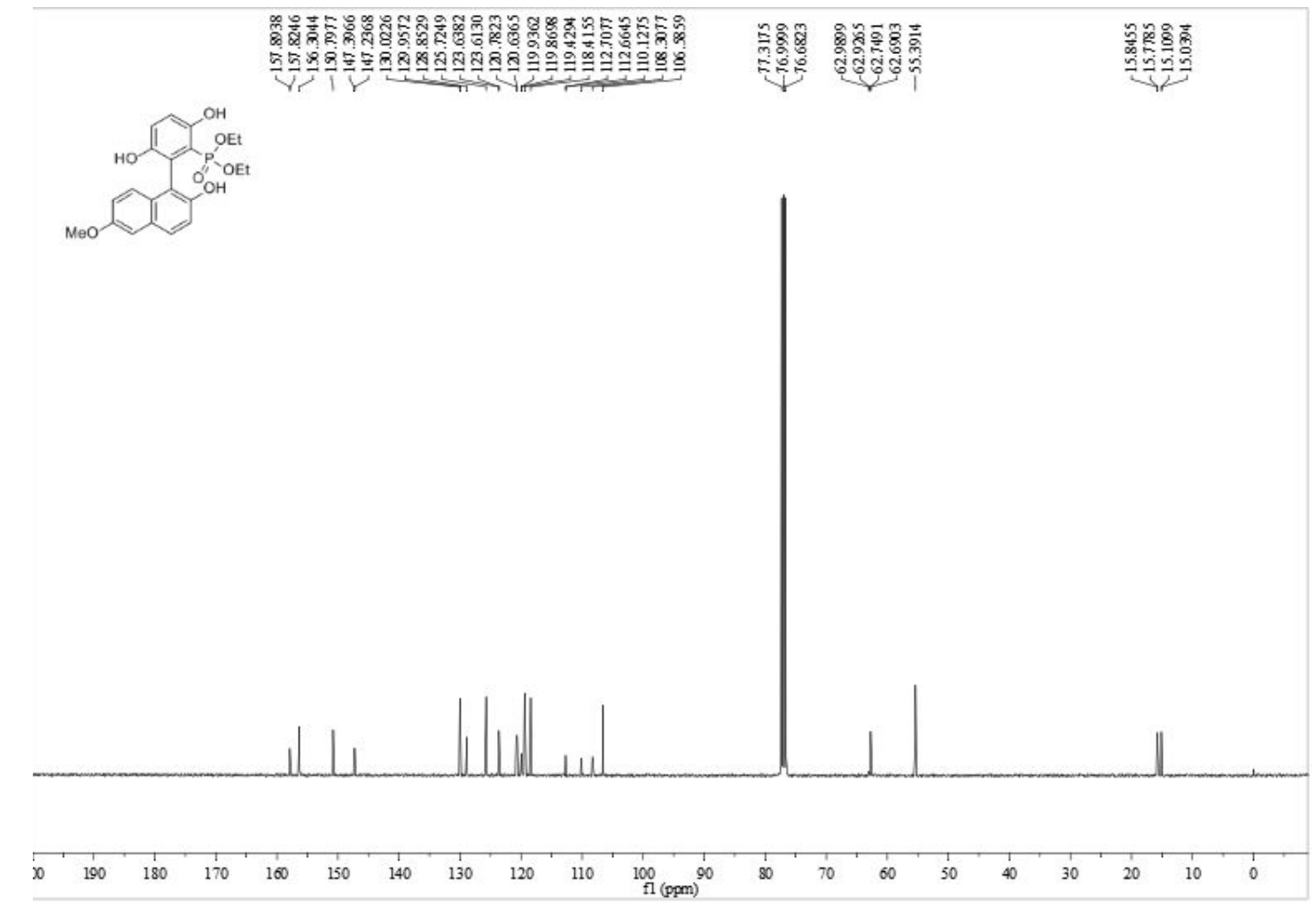


${ }^{1} \mathrm{H}$ NMR for compound $\mathbf{4 c f}\left(400 \mathrm{MHz}, \mathrm{CDCl}_{3}\right)$

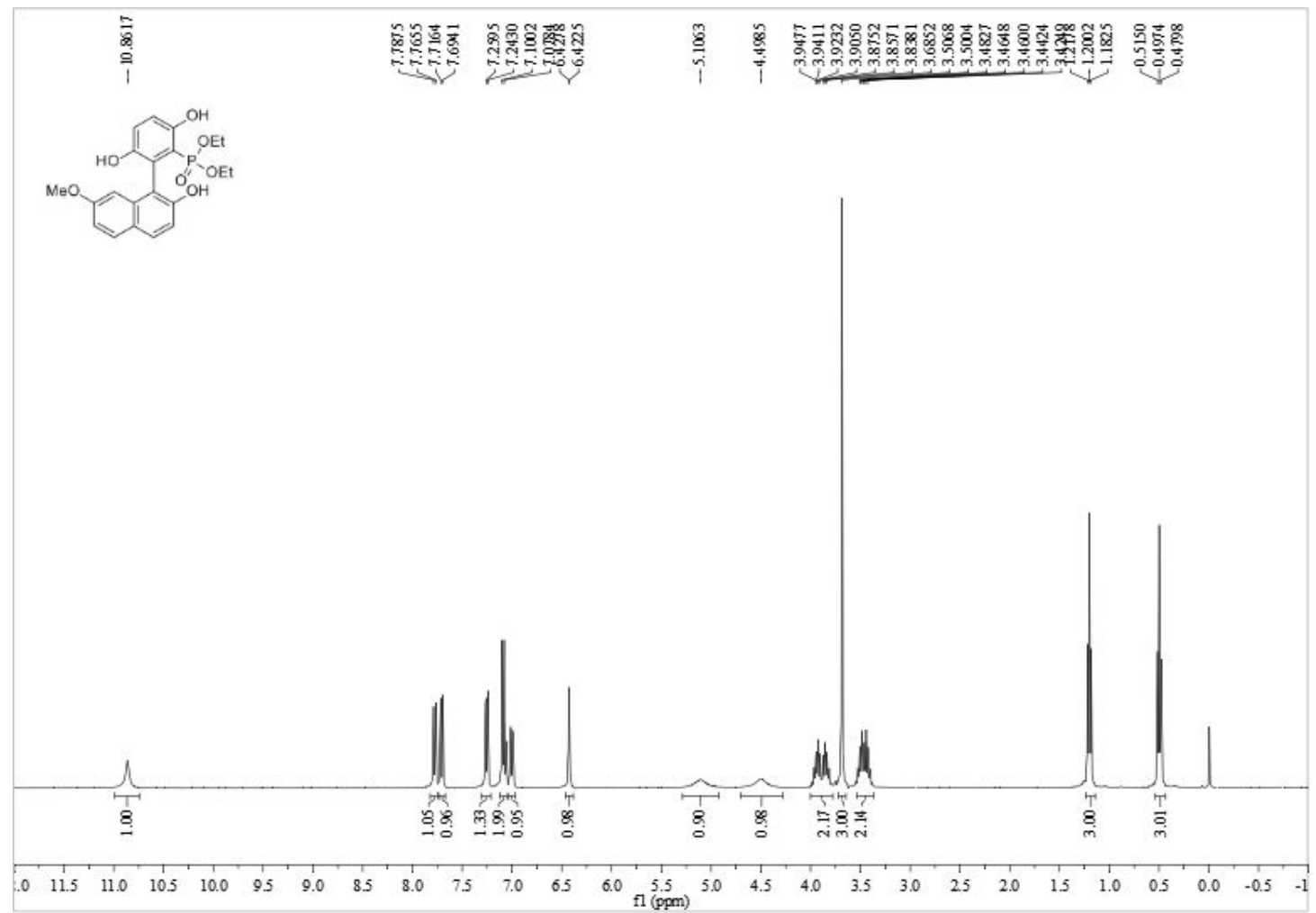

${ }^{31} \mathrm{P}$ NMR for compound $4 \mathbf{c f}\left(162 \mathrm{MHz}, \mathrm{CDCl}_{3}\right)$

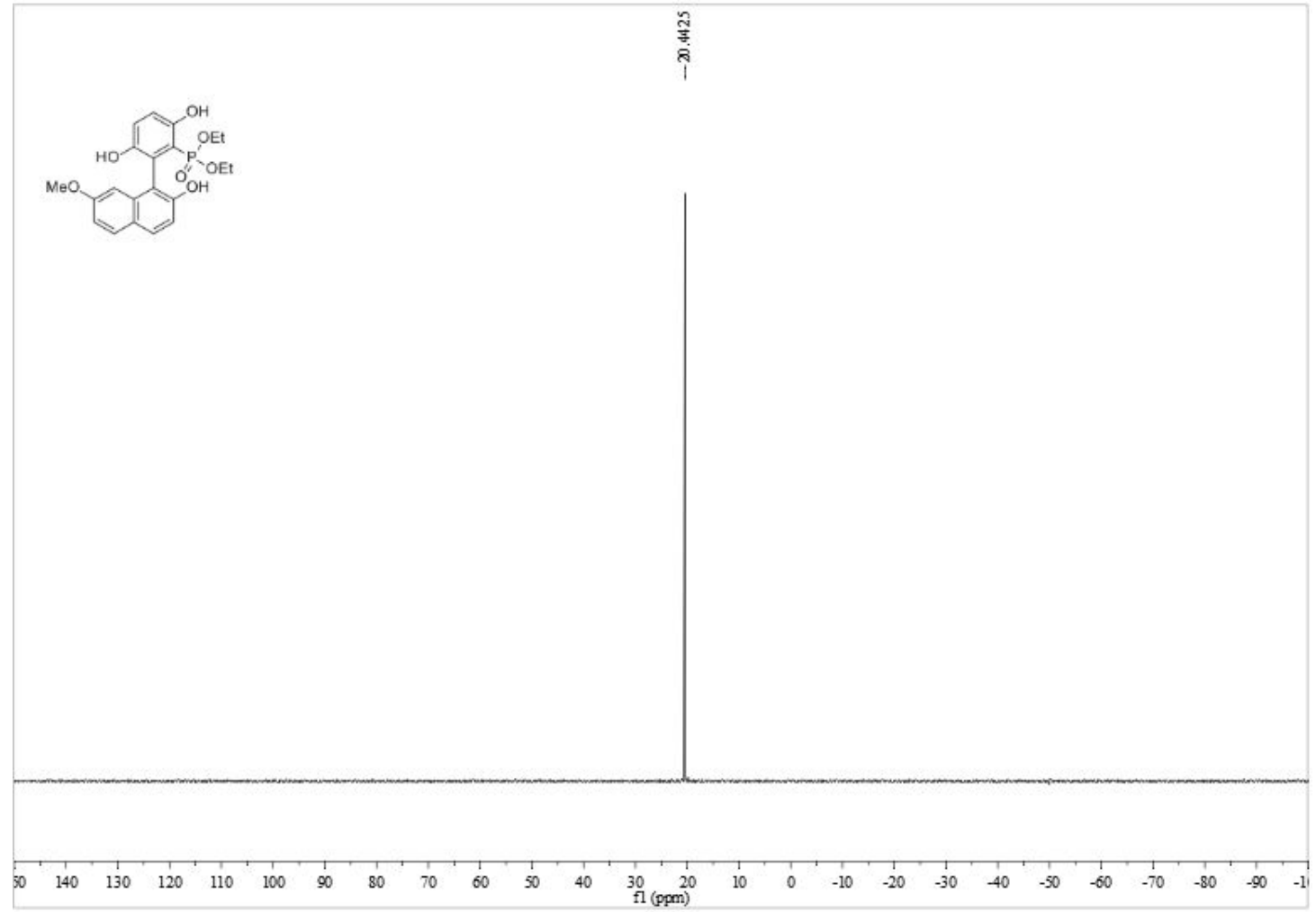


${ }^{13} \mathrm{C}\left\{{ }^{1} \mathrm{H}\right\}$ NMR for compound $4 \mathbf{c f}\left(101 \mathrm{MHz}, \mathrm{CDCl}_{3}\right)$

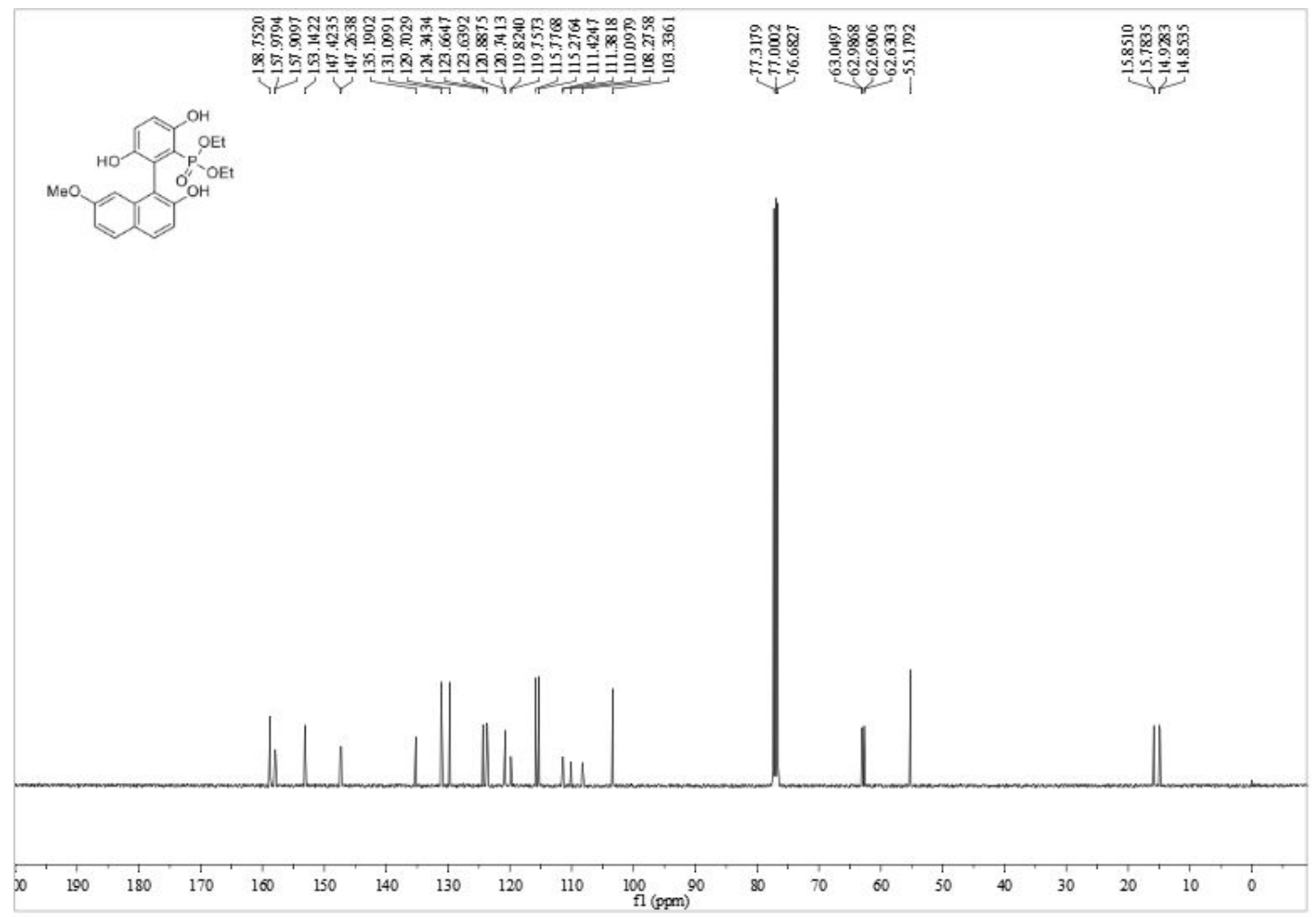

${ }^{1} \mathrm{H}$ NMR for compound $4 \mathbf{c g}\left(400 \mathrm{MHz}, \mathrm{CDCl}_{3}\right.$ )

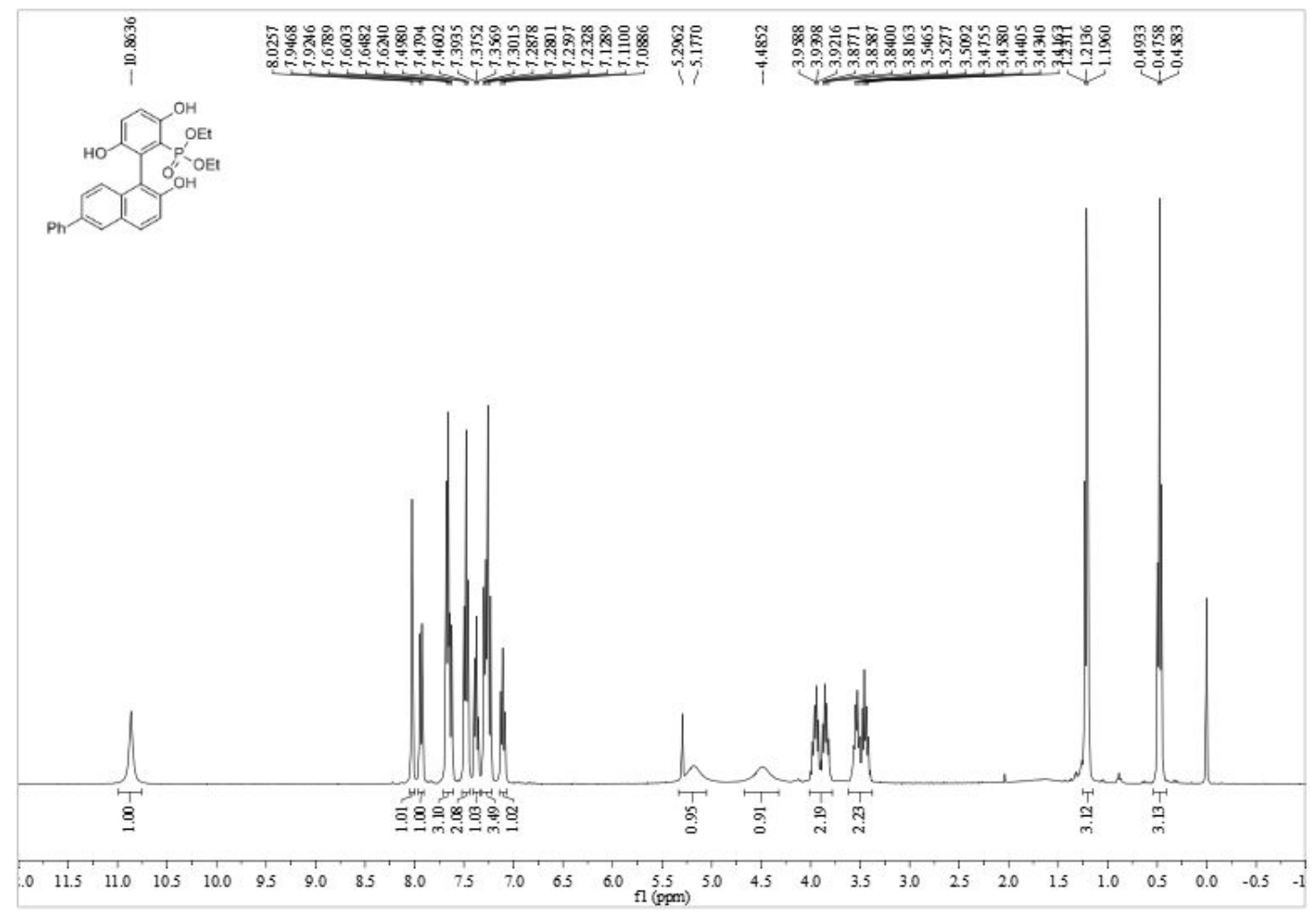


${ }^{31} \mathrm{P}$ NMR for compound 4cg (162 $\left.\mathrm{MHz}, \mathrm{CDCl}_{3}\right)$

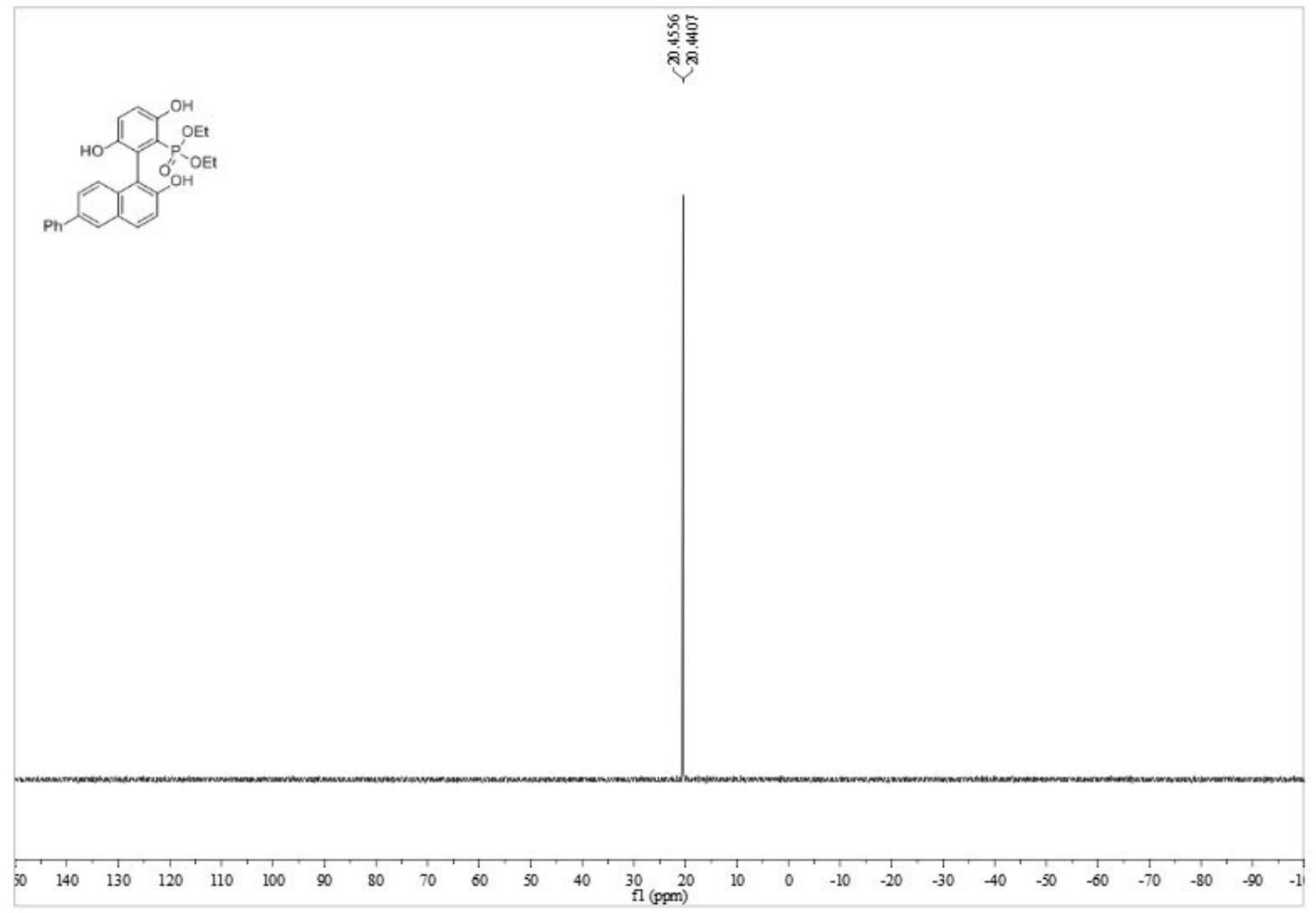

${ }^{13} \mathrm{C}\left\{{ }^{1} \mathrm{H}\right\}$ NMR for compound $\mathbf{4 c g}\left(101 \mathrm{MHz}, \mathrm{CDCl}_{3}\right)$

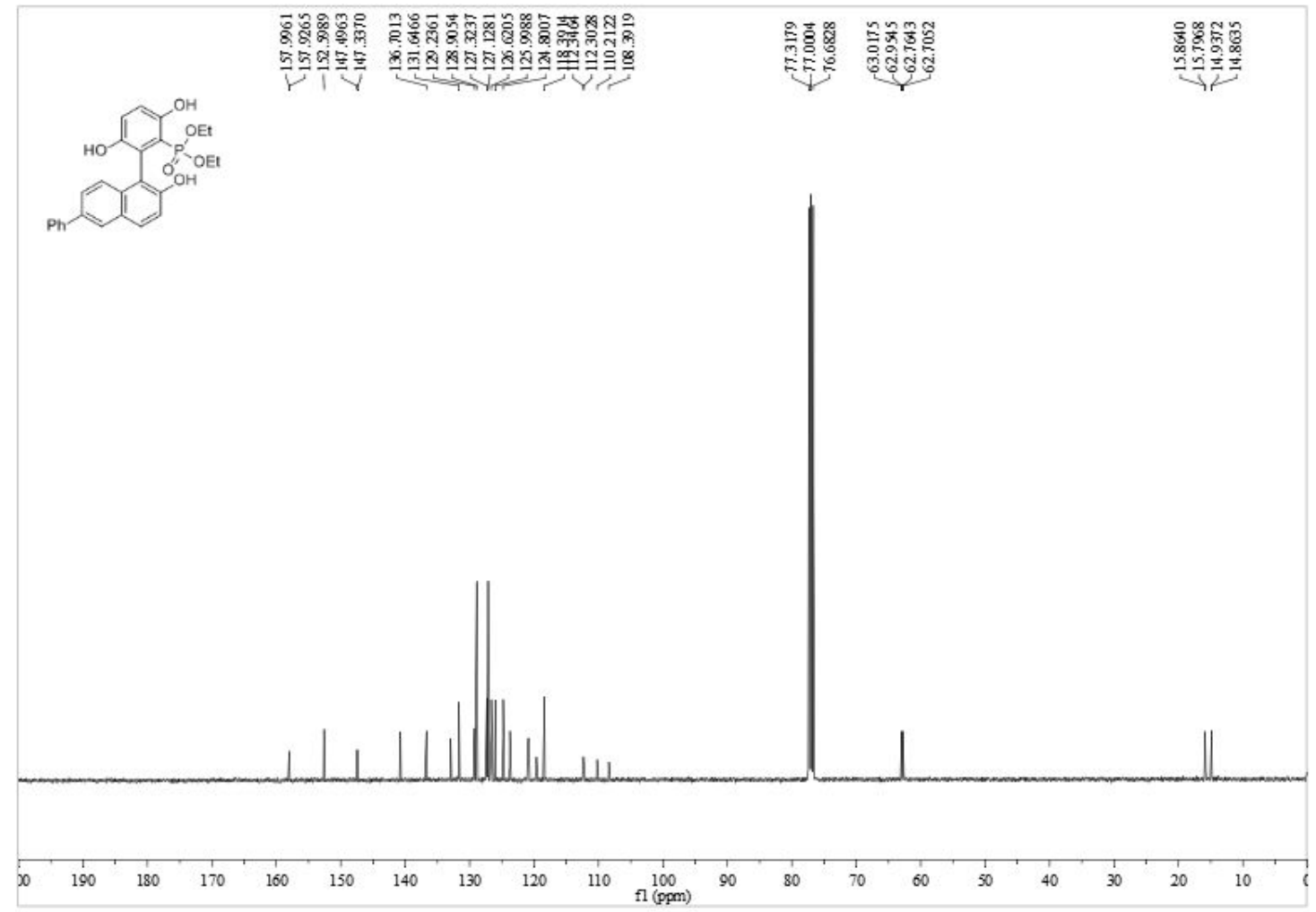


${ }^{1} \mathrm{H}$ NMR for compound $\mathbf{4 c h}\left(400 \mathrm{MHz}, \mathrm{CDCl}_{3}\right)$

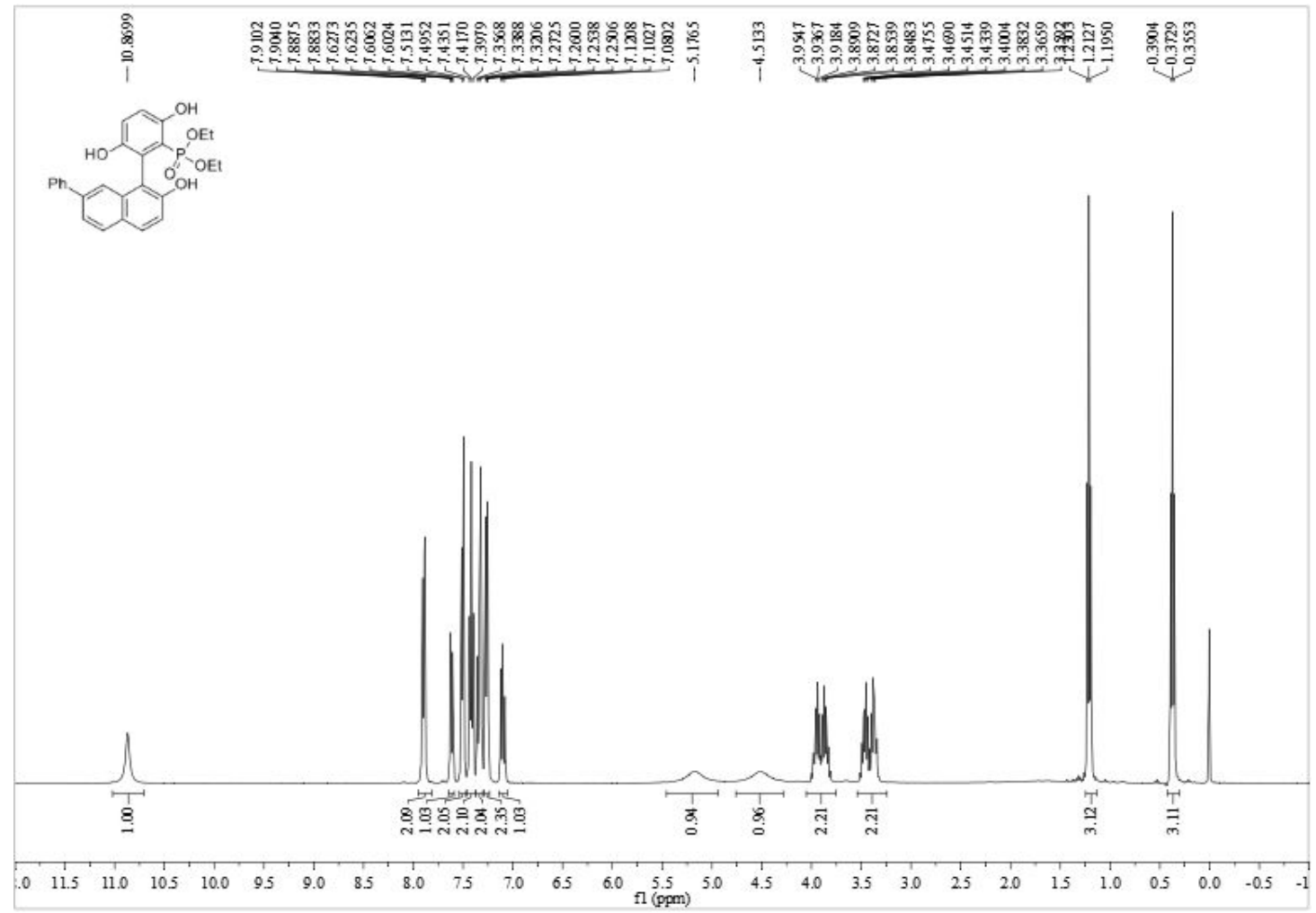

${ }^{31} \mathrm{P}$ NMR for compound $4 \mathbf{c h}\left(162 \mathrm{MHz}, \mathrm{CDCl}_{3}\right)$

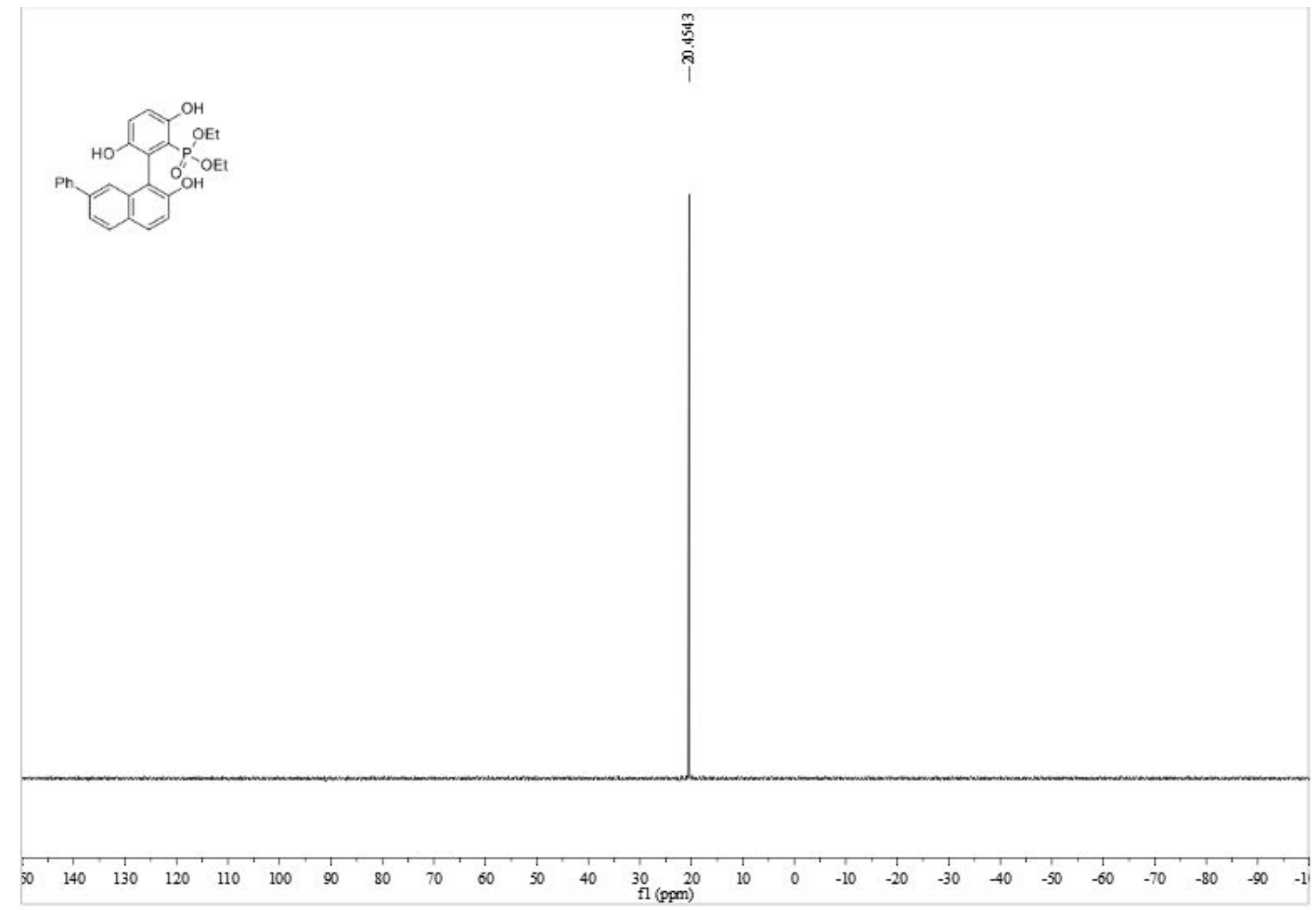


${ }^{13} \mathrm{C}\left\{{ }^{1} \mathrm{H}\right\}$ NMR for compound $\mathbf{4 c h}\left(101 \mathrm{MHz}, \mathrm{CDCl}_{3}\right)$

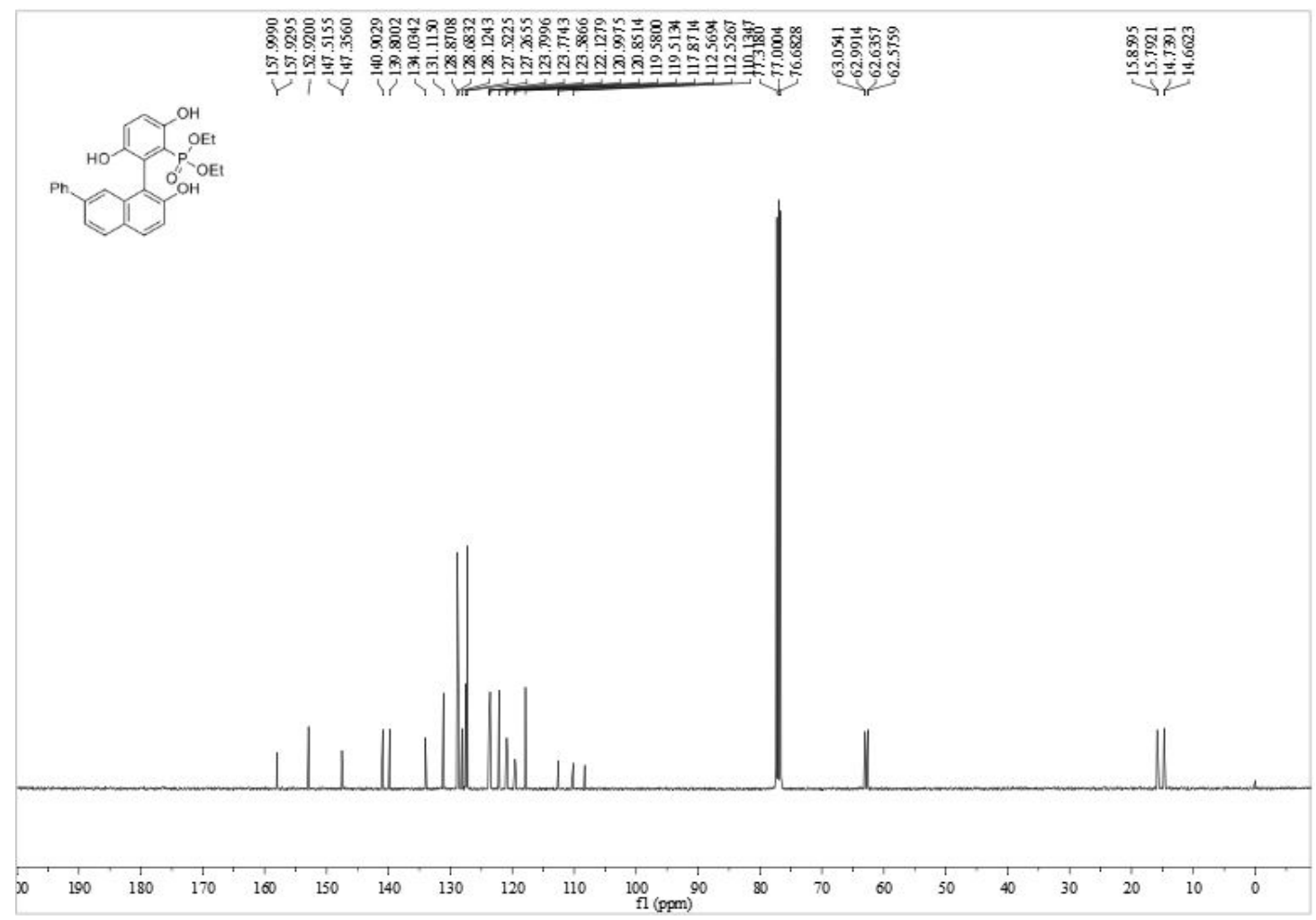

${ }^{1} \mathrm{H}$ NMR for compound $4 \mathbf{c i}\left(400 \mathrm{MHz}, \mathrm{CDCl}_{3}\right.$ )

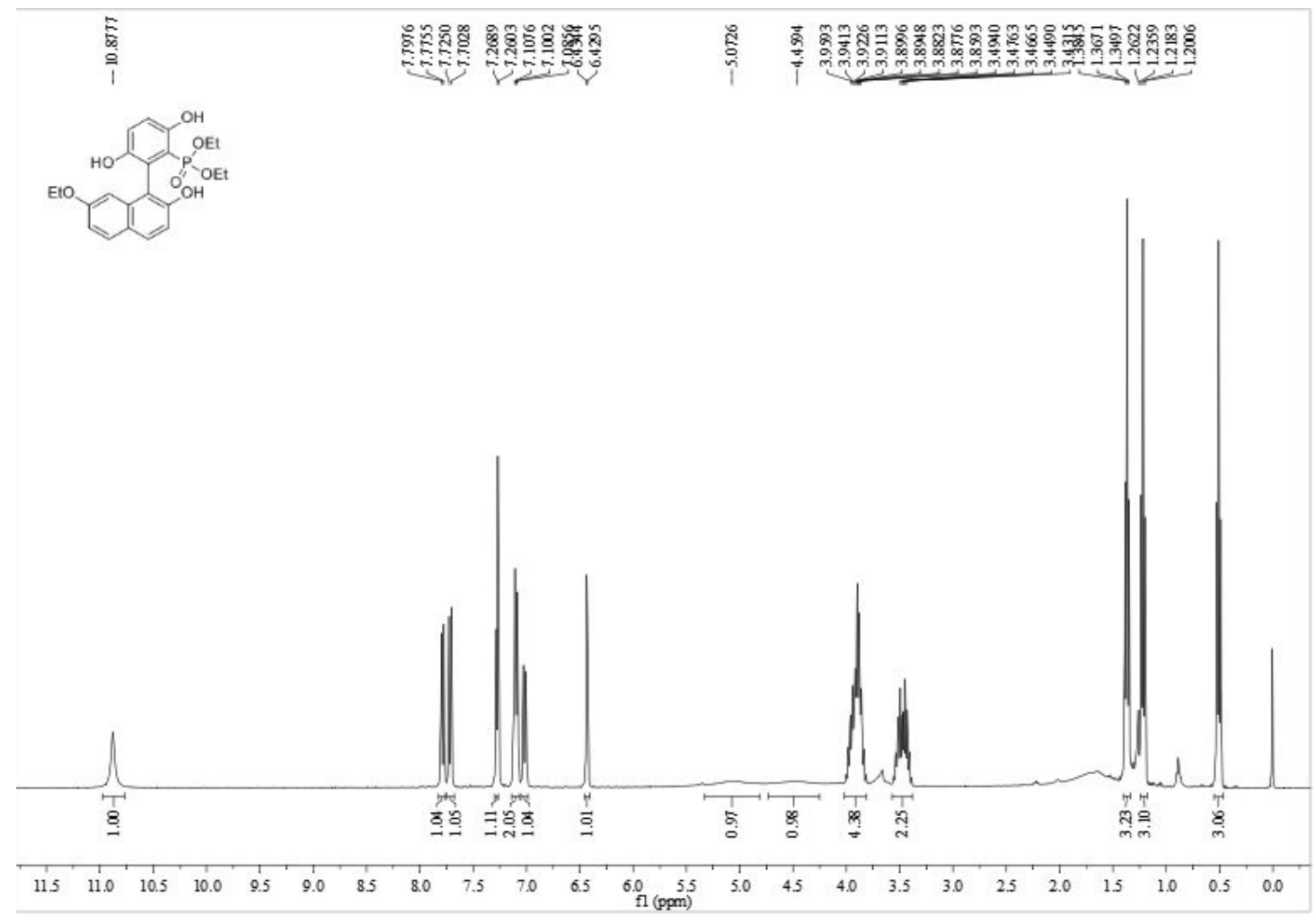


${ }^{31} \mathrm{P}$ NMR for compound 4ci (162 MHz, $\mathrm{CDCl}_{3}$ )

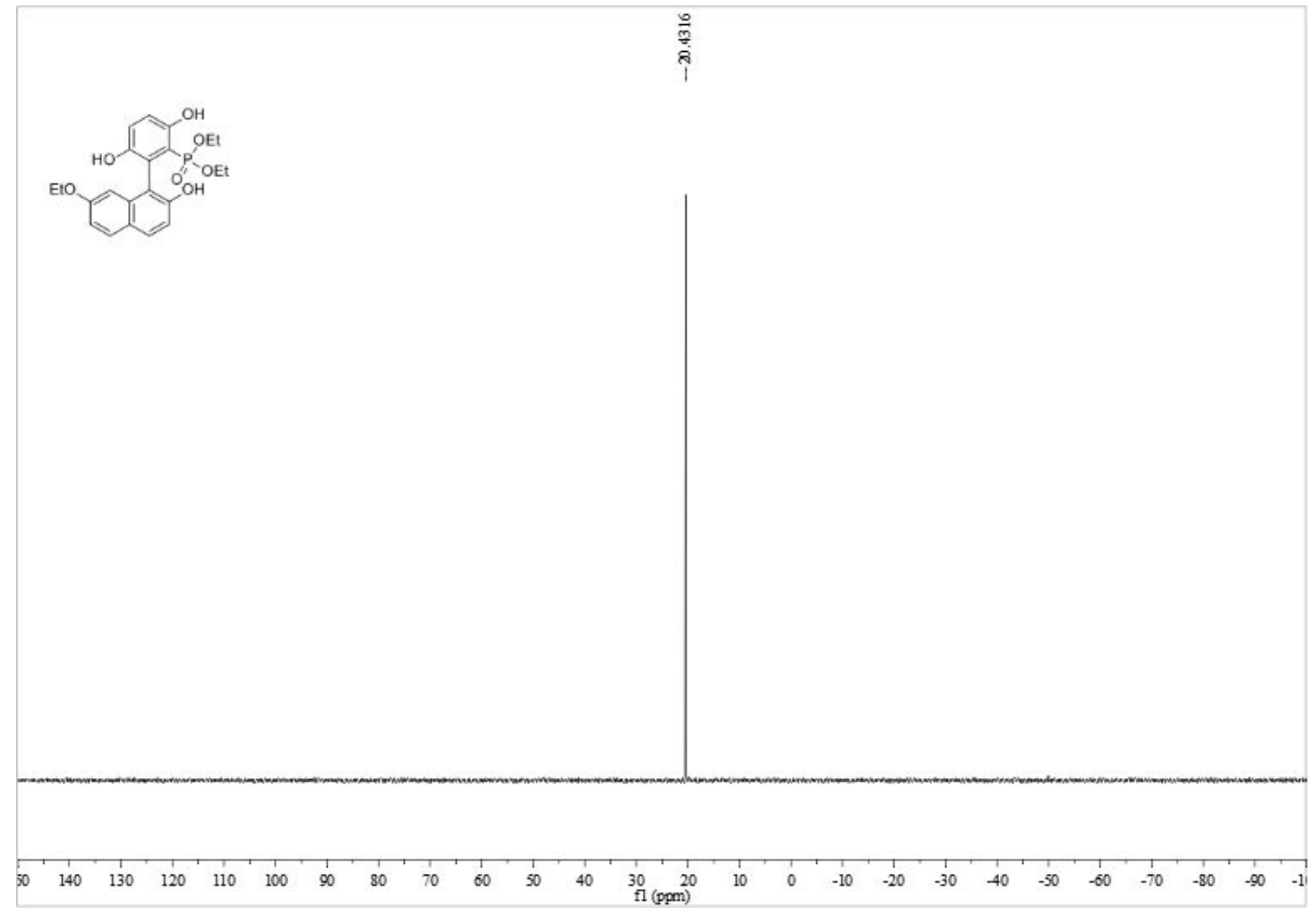

${ }^{13} \mathrm{C}\left\{{ }^{1} \mathrm{H}\right\}$ NMR for compound 4ci $\left(101 \mathrm{MHz}, \mathrm{CDCl}_{3}\right)$

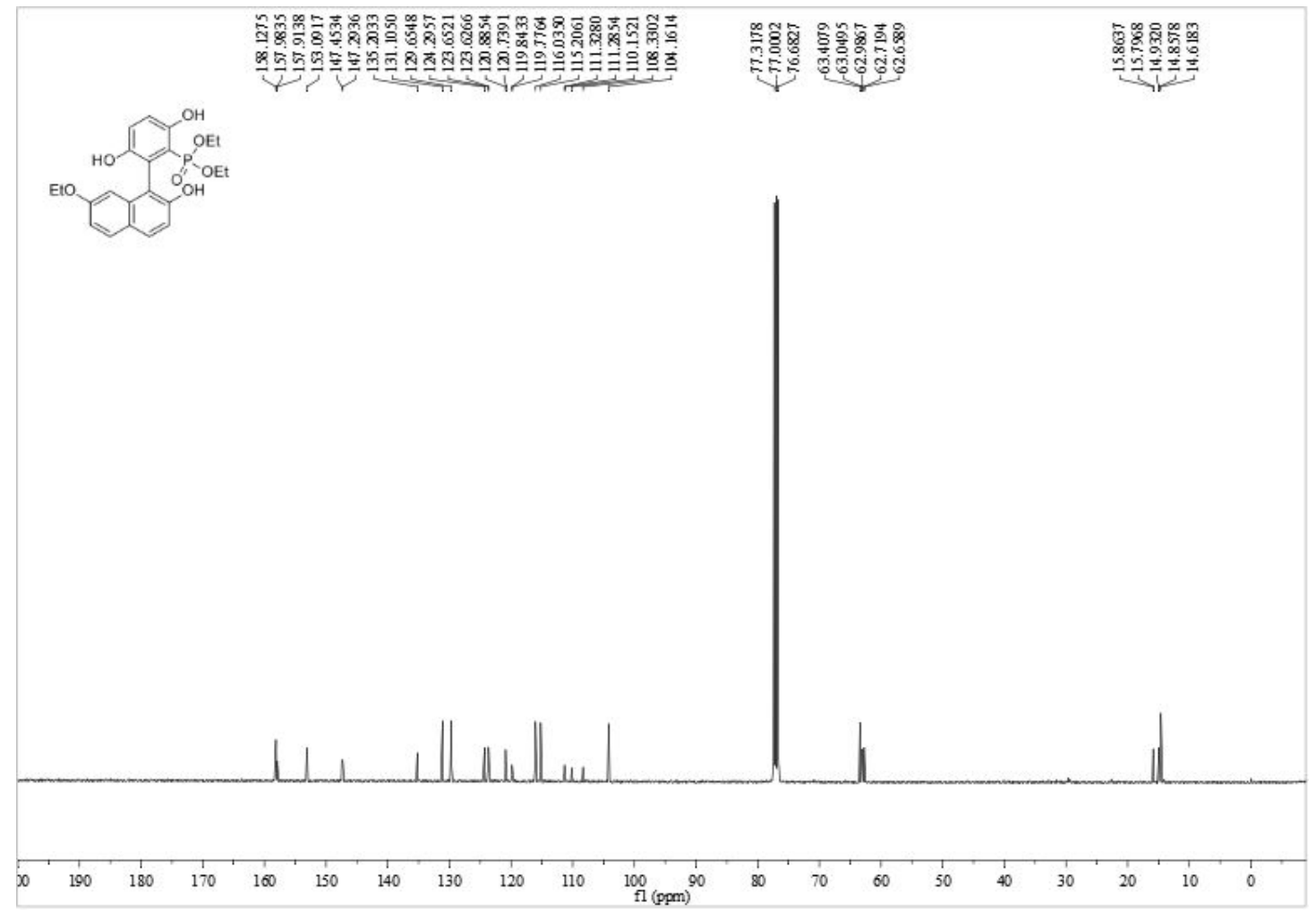


${ }^{1} \mathrm{H}$ NMR for compound 4cj (400 MHz, $\mathrm{CDCl}_{3}$ )

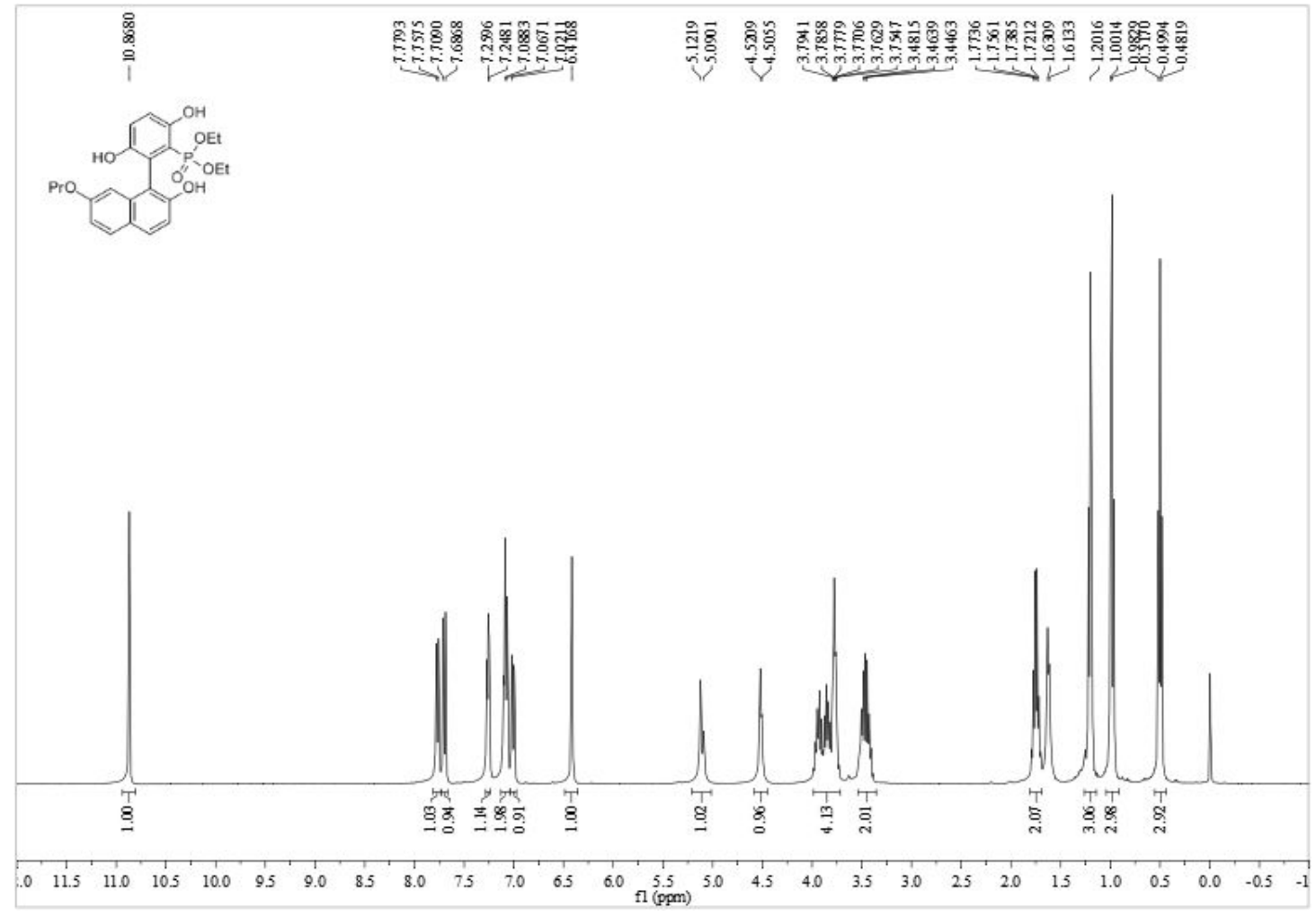

${ }^{31} \mathrm{P}$ NMR for compound 4cj (162 MHz, $\mathrm{CDCl}_{3}$ )

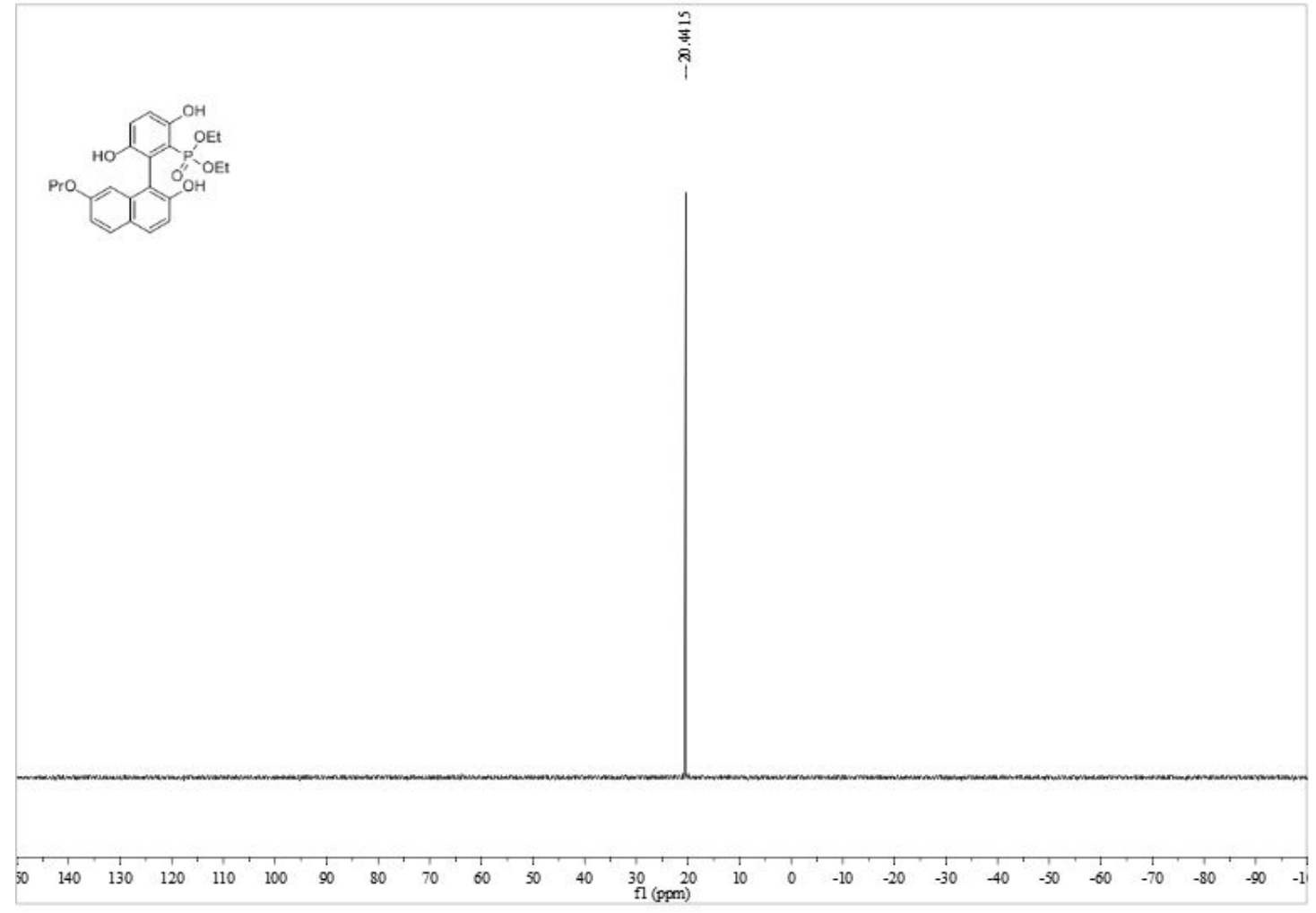


${ }^{13} \mathrm{C}\left\{{ }^{1} \mathrm{H}\right\}$ NMR for compound $\mathbf{4} \mathbf{j}\left(101 \mathrm{MHz}, \mathrm{CDCl}_{3}\right)$

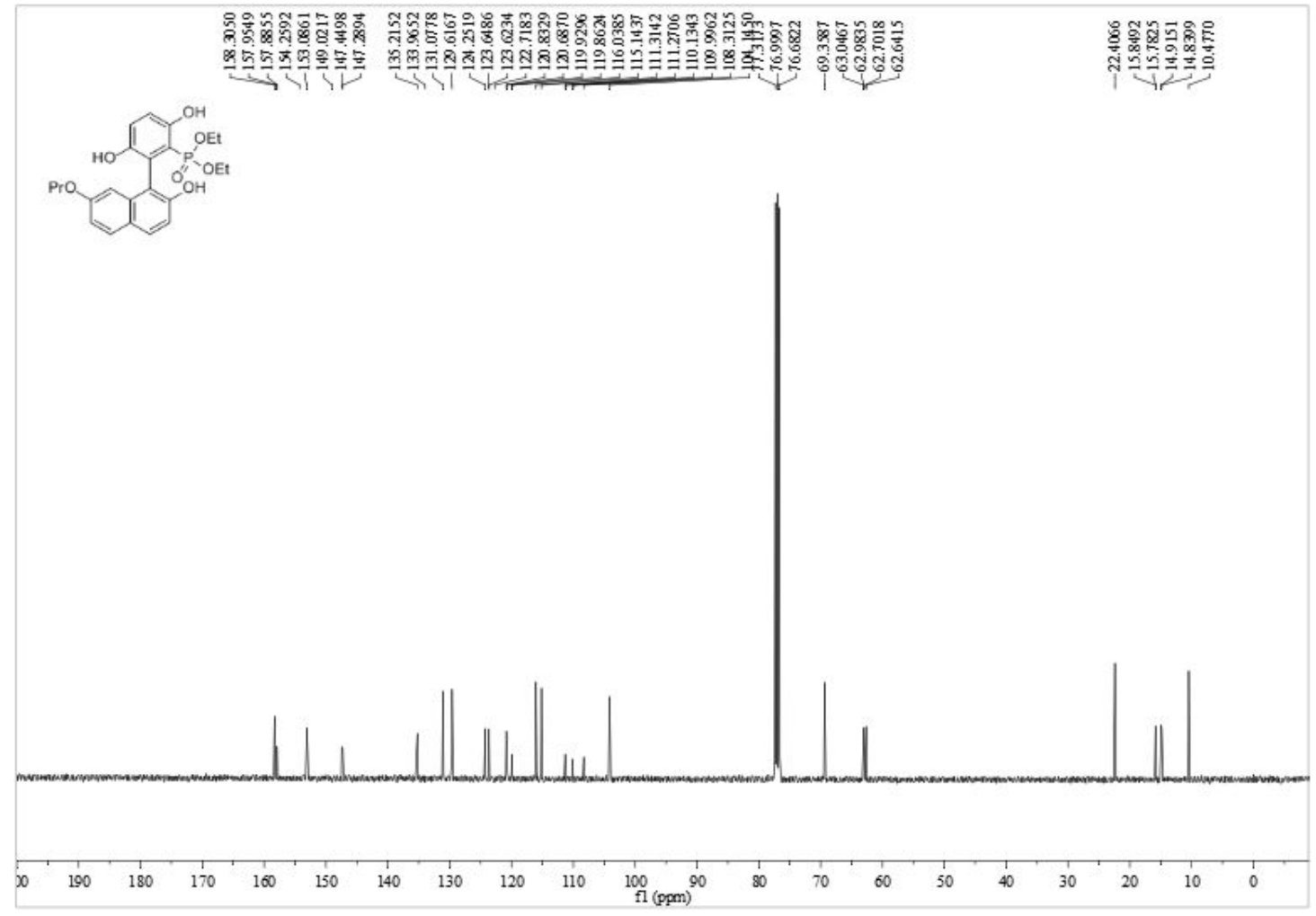

${ }^{1} \mathrm{H}$ NMR for compound $\mathbf{4} \mathbf{c k}\left(400 \mathrm{MHz}, \mathrm{CDCl}_{3}\right.$ )

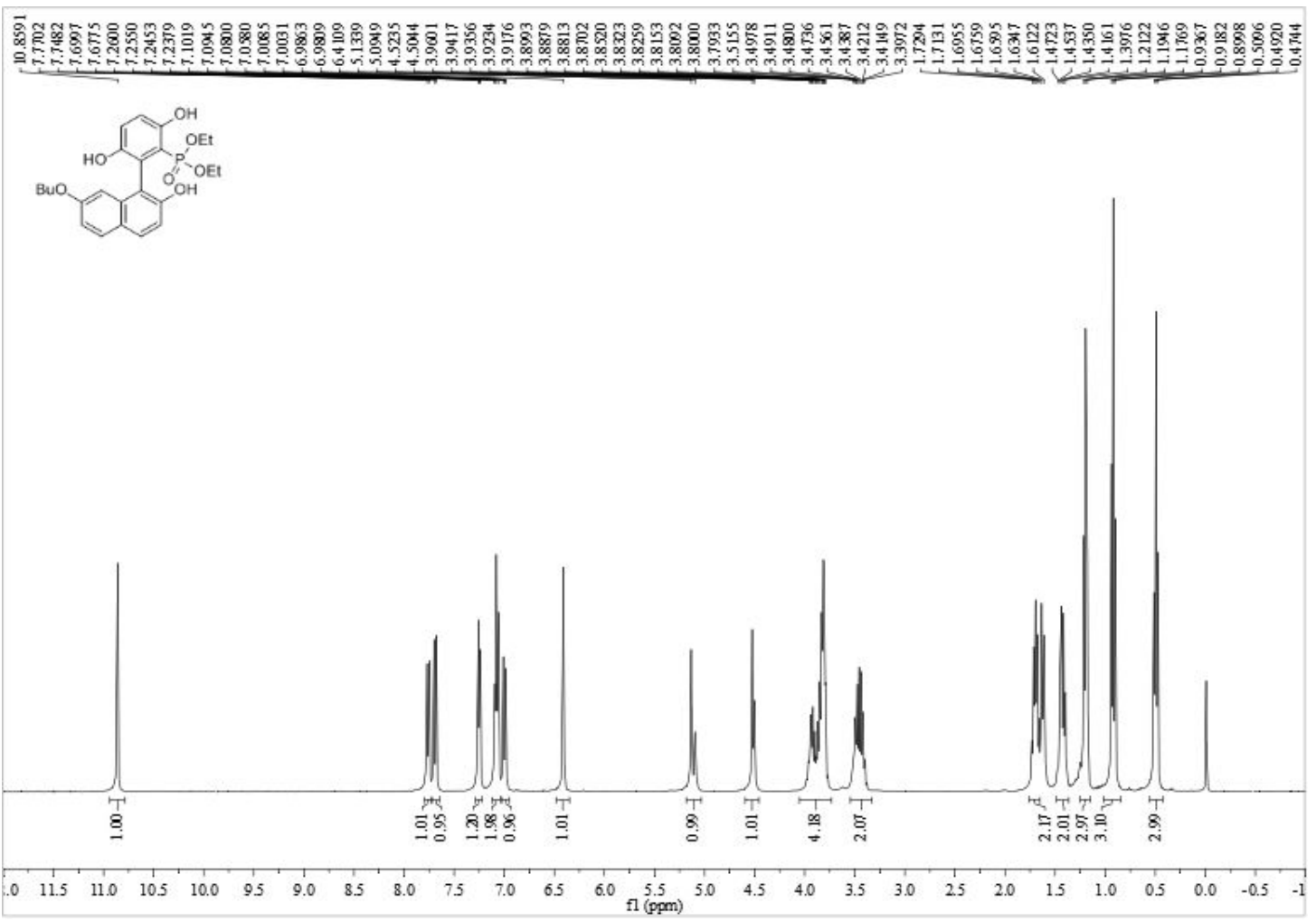


${ }^{31} \mathrm{P}$ NMR for compound 4ck (162 MHz, $\left.\mathrm{CDCl}_{3}\right)$

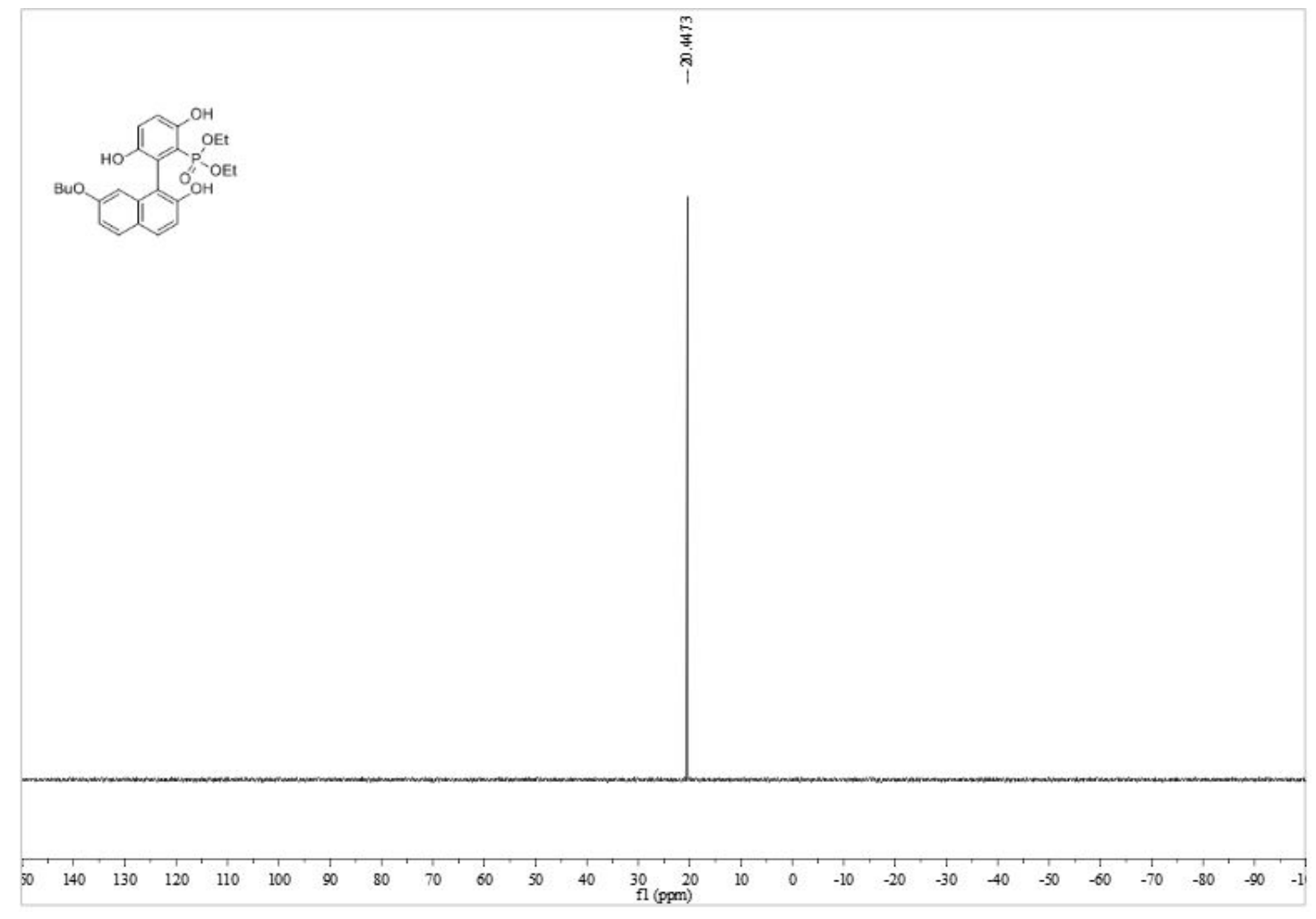

${ }^{13} \mathrm{C}\left\{{ }^{1} \mathrm{H}\right\}$ NMR for compound 4ck (101 MHz, $\left.\mathrm{CDCl}_{3}\right)$

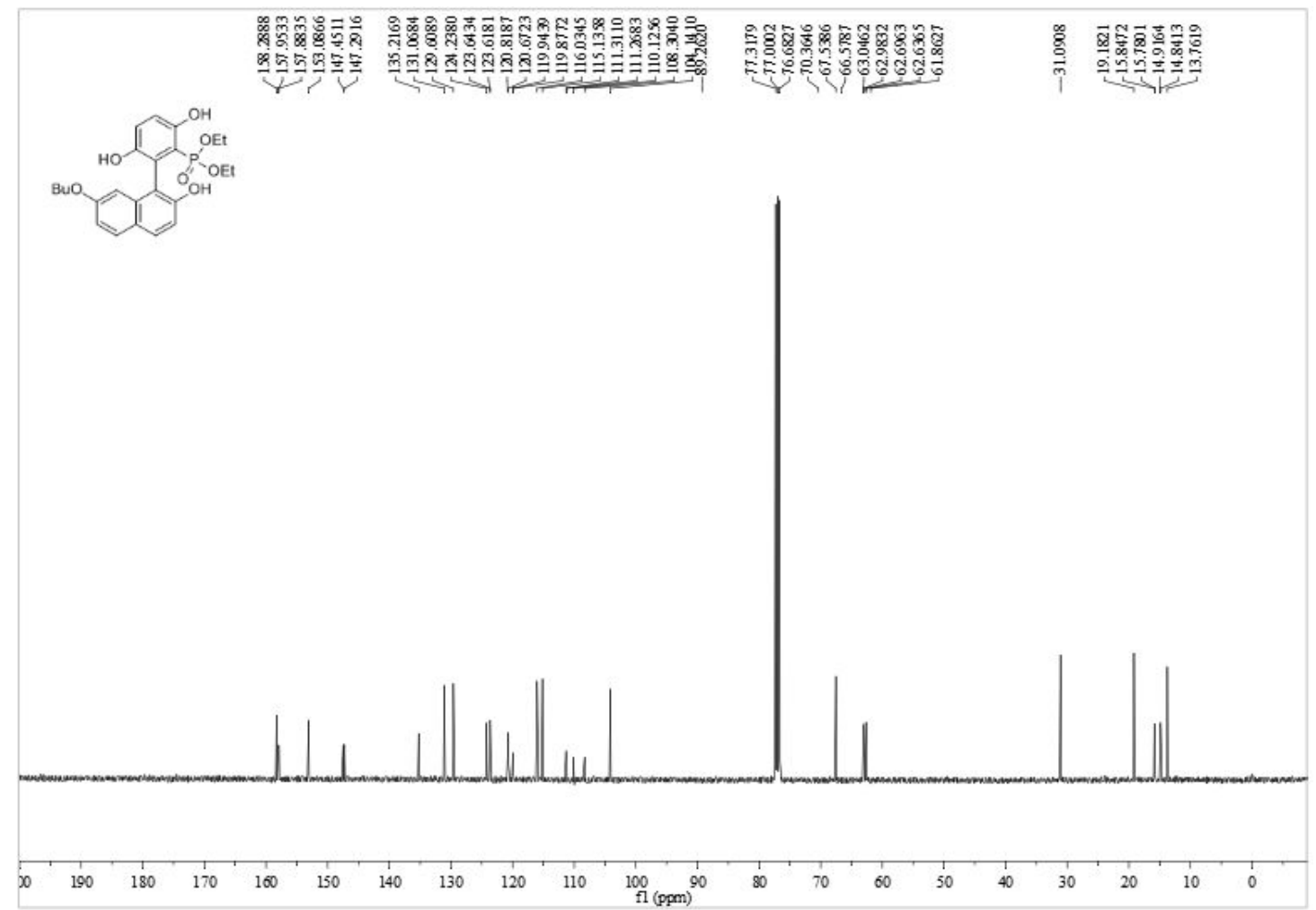


${ }^{1} \mathrm{H}$ NMR for compound $\mathbf{4 c l}\left(400 \mathrm{MHz}, \mathrm{CDCl}_{3}\right.$ )

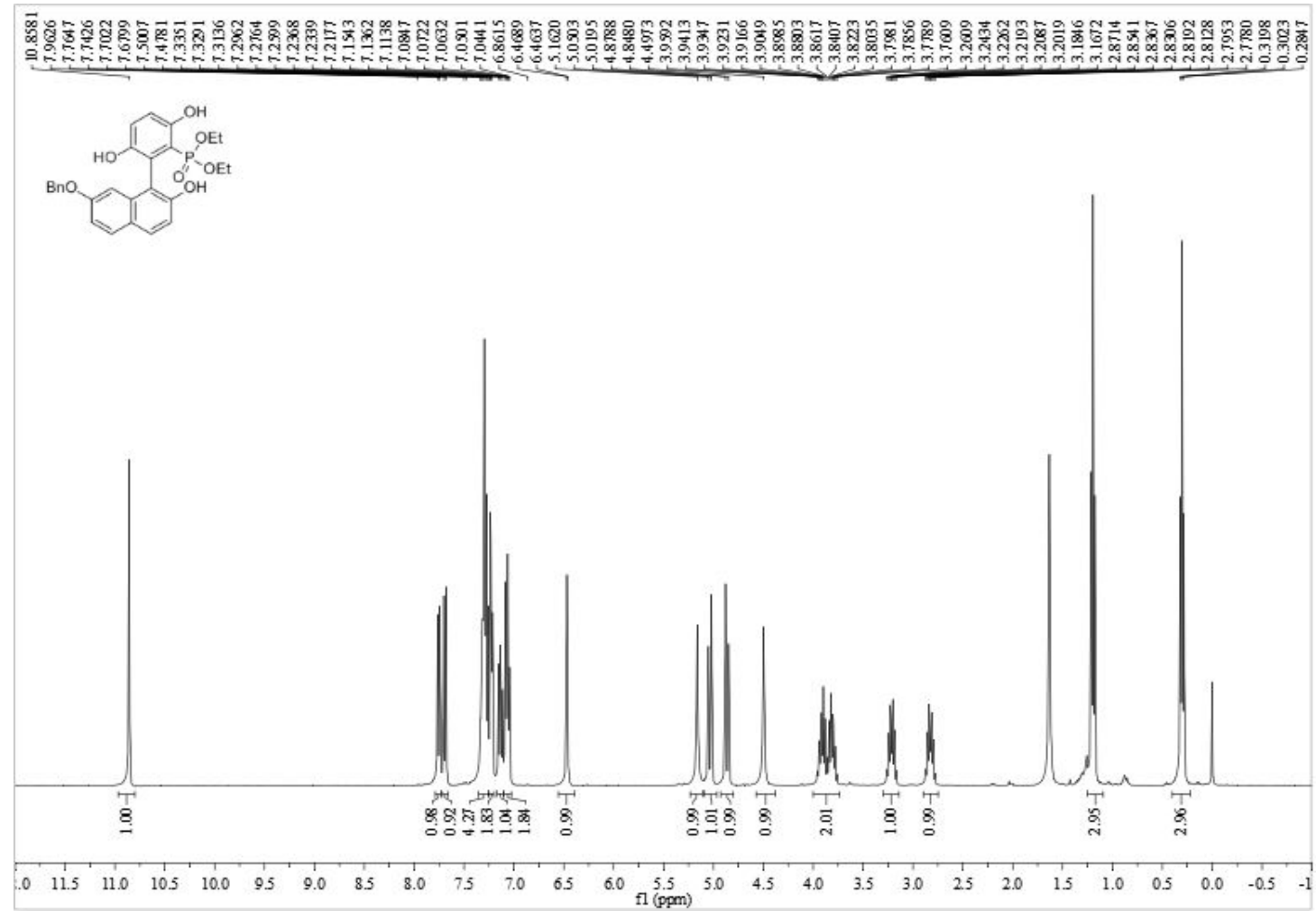

${ }^{31} \mathrm{P}$ NMR for compound $4 \mathrm{c}\left(162 \mathrm{MHz}, \mathrm{CDCl}_{3}\right)$

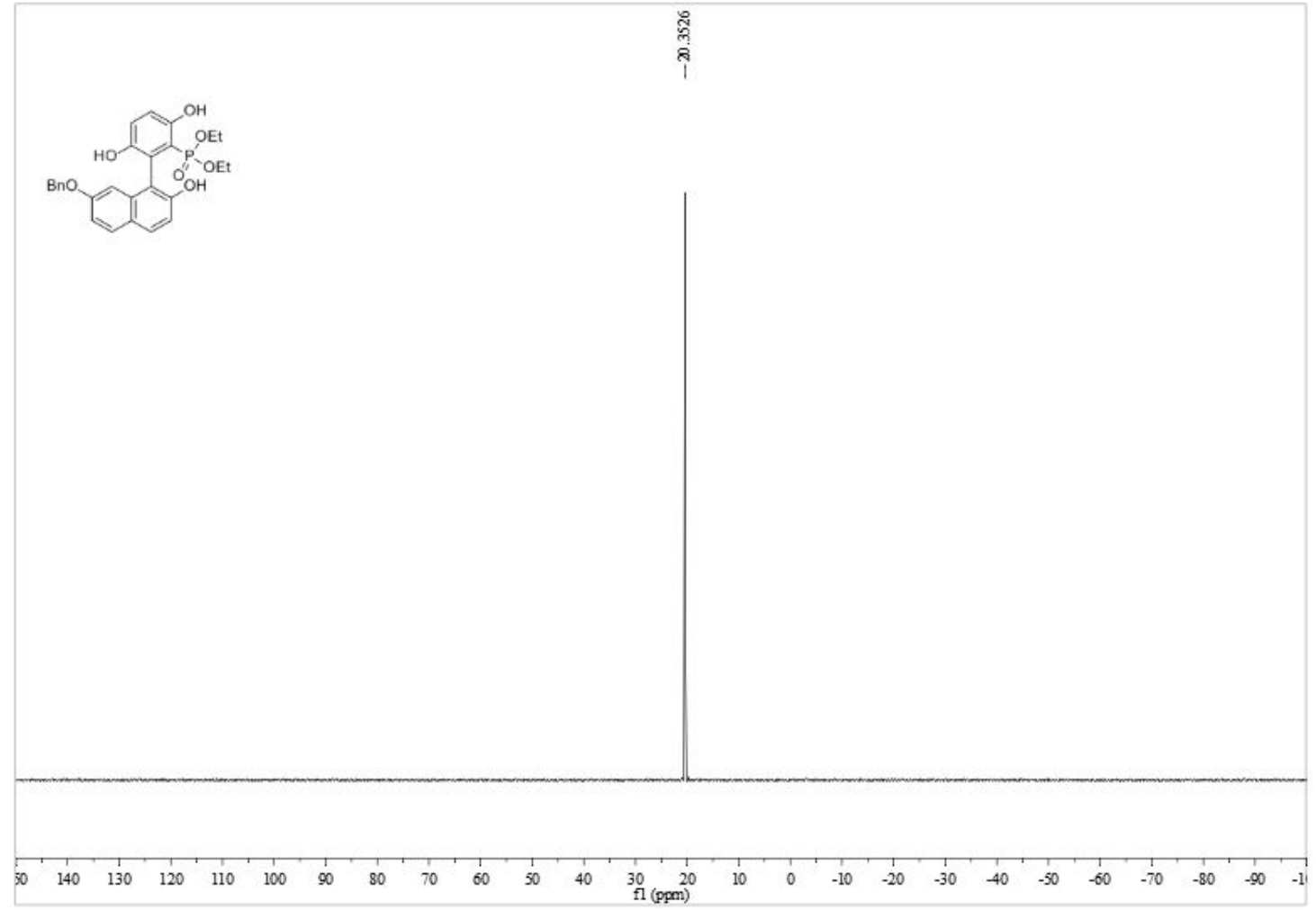


${ }^{13} \mathrm{C}\left\{{ }^{1} \mathrm{H}\right\}$ NMR for compound $4 \mathbf{4 l}\left(101 \mathrm{MHz}, \mathrm{CDCl}_{3}\right)$

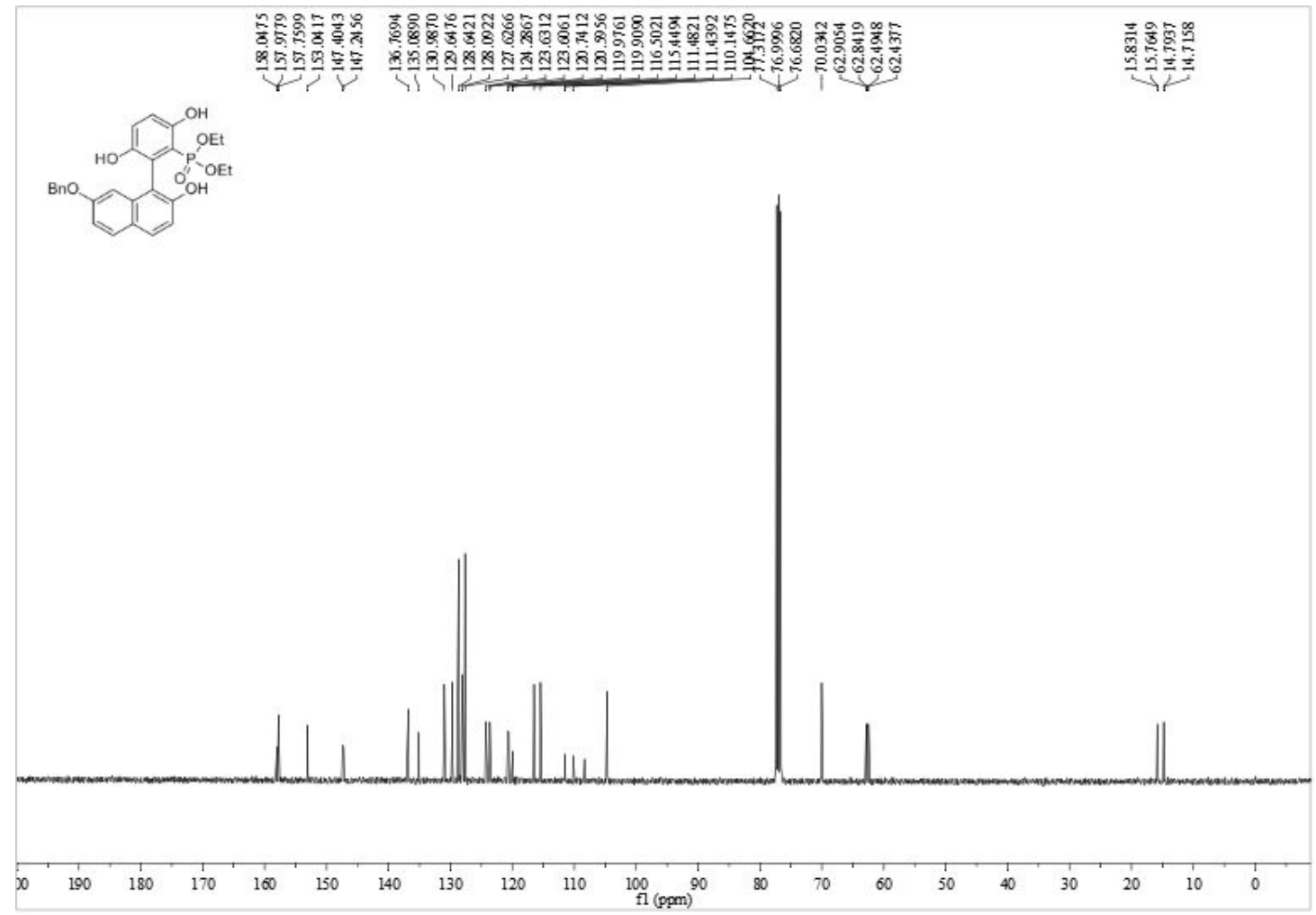

${ }^{1} \mathrm{H}$ NMR for compound $\mathbf{4 c m}\left(400 \mathrm{MHz}, \mathrm{CDCl}_{3}\right)$

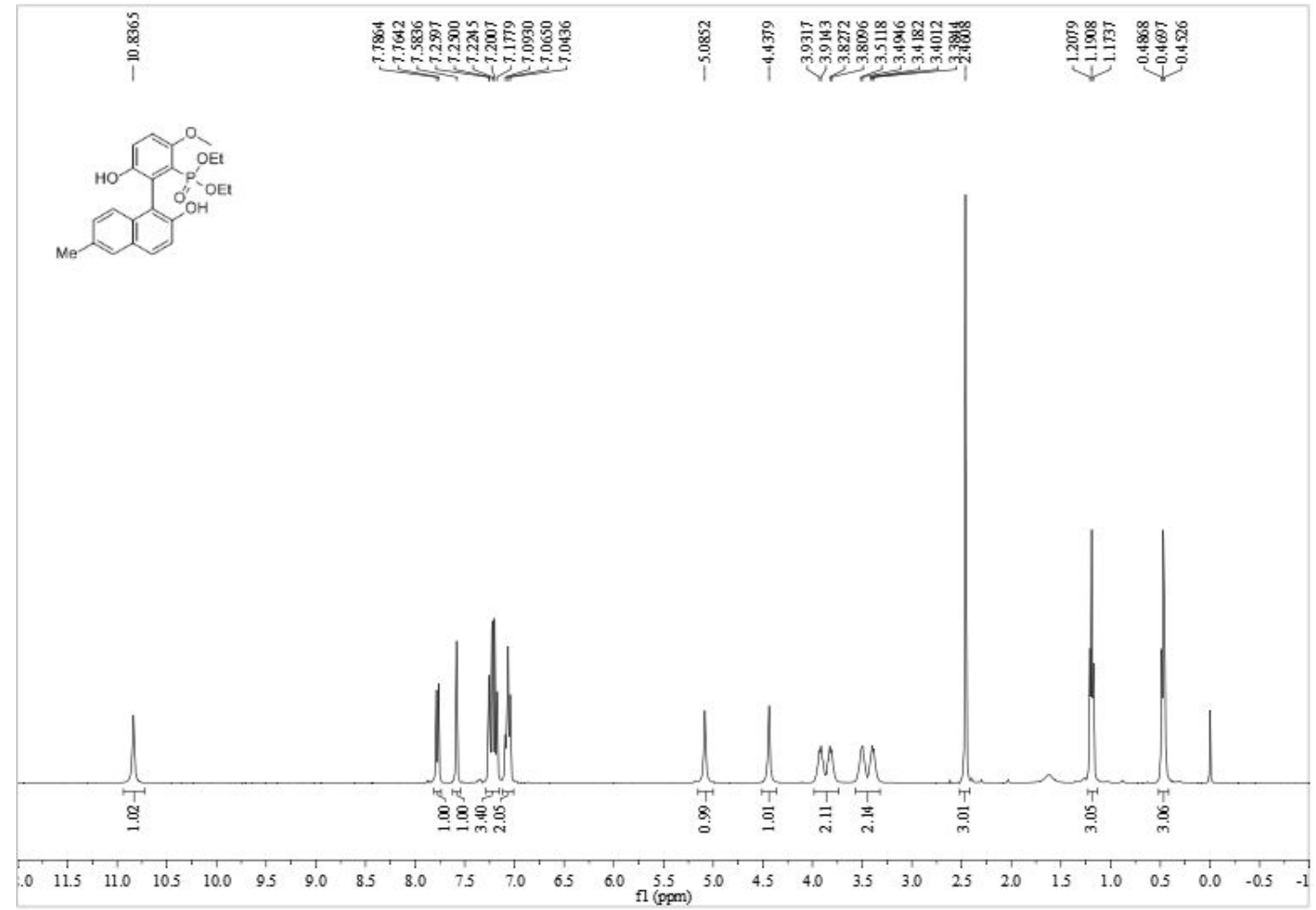


${ }^{31} \mathrm{P}$ NMR for compound $4 \mathbf{c m}\left(162 \mathrm{MHz}, \mathrm{CDCl}_{3}\right)$

ฺิ
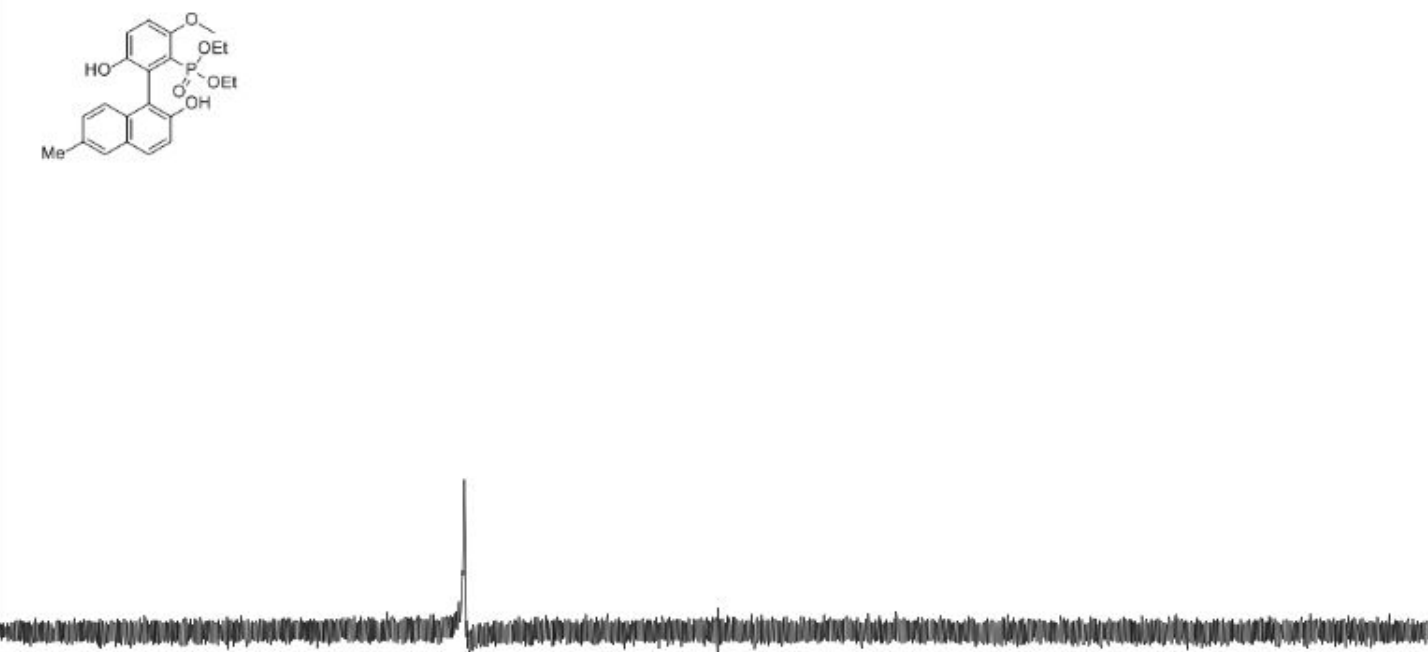

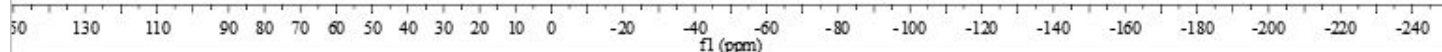

${ }^{13} \mathrm{C}\left\{{ }^{1} \mathrm{H}\right\}$ NMR for compound $\mathbf{4 c m}\left(101 \mathrm{MHz}, \mathrm{CDCl}_{3}\right)$

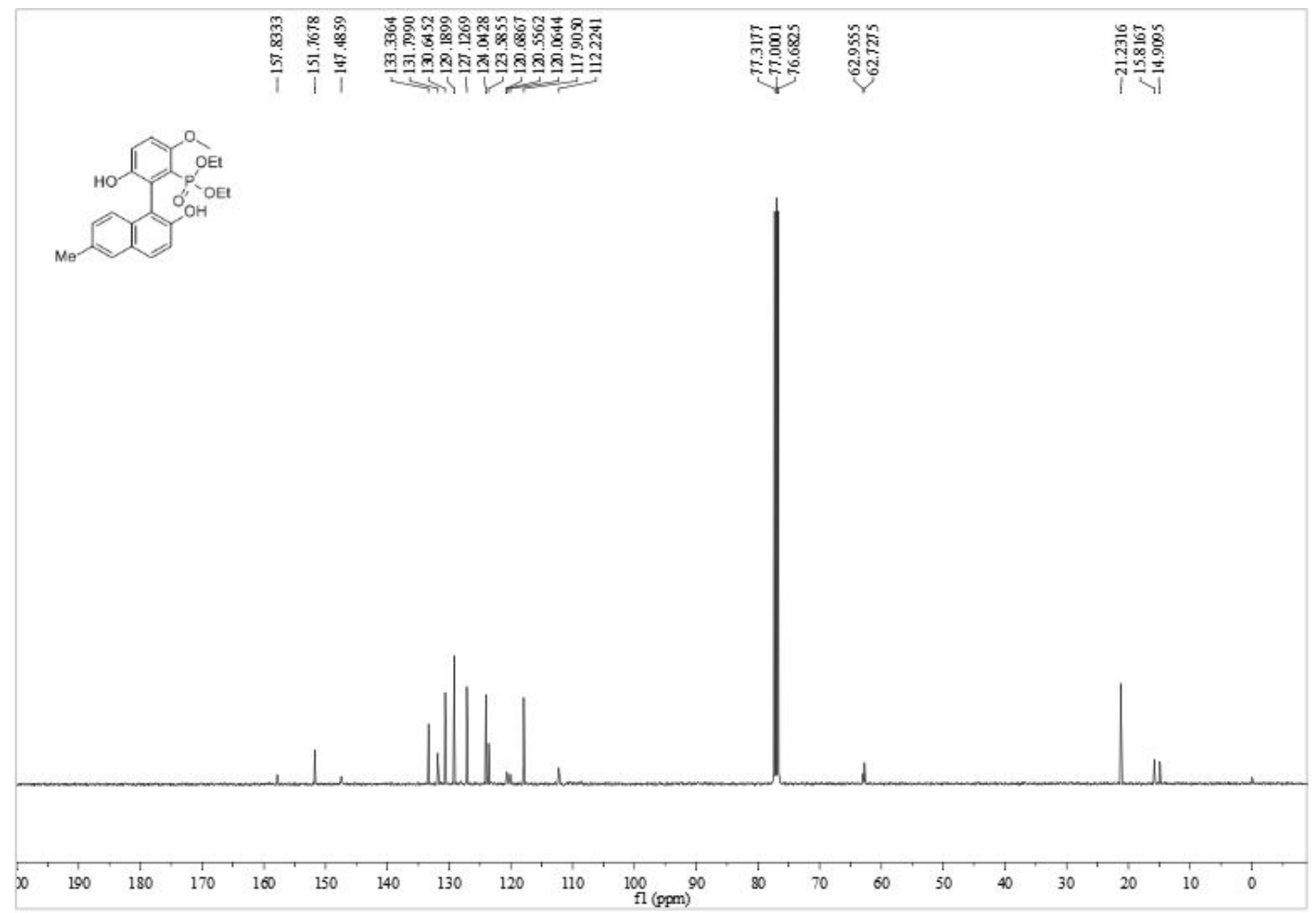


${ }^{1} \mathrm{H}$ NMR for compound $4 \mathbf{c n}\left(400 \mathrm{MHz}, \mathrm{CDCl}_{3}\right)$

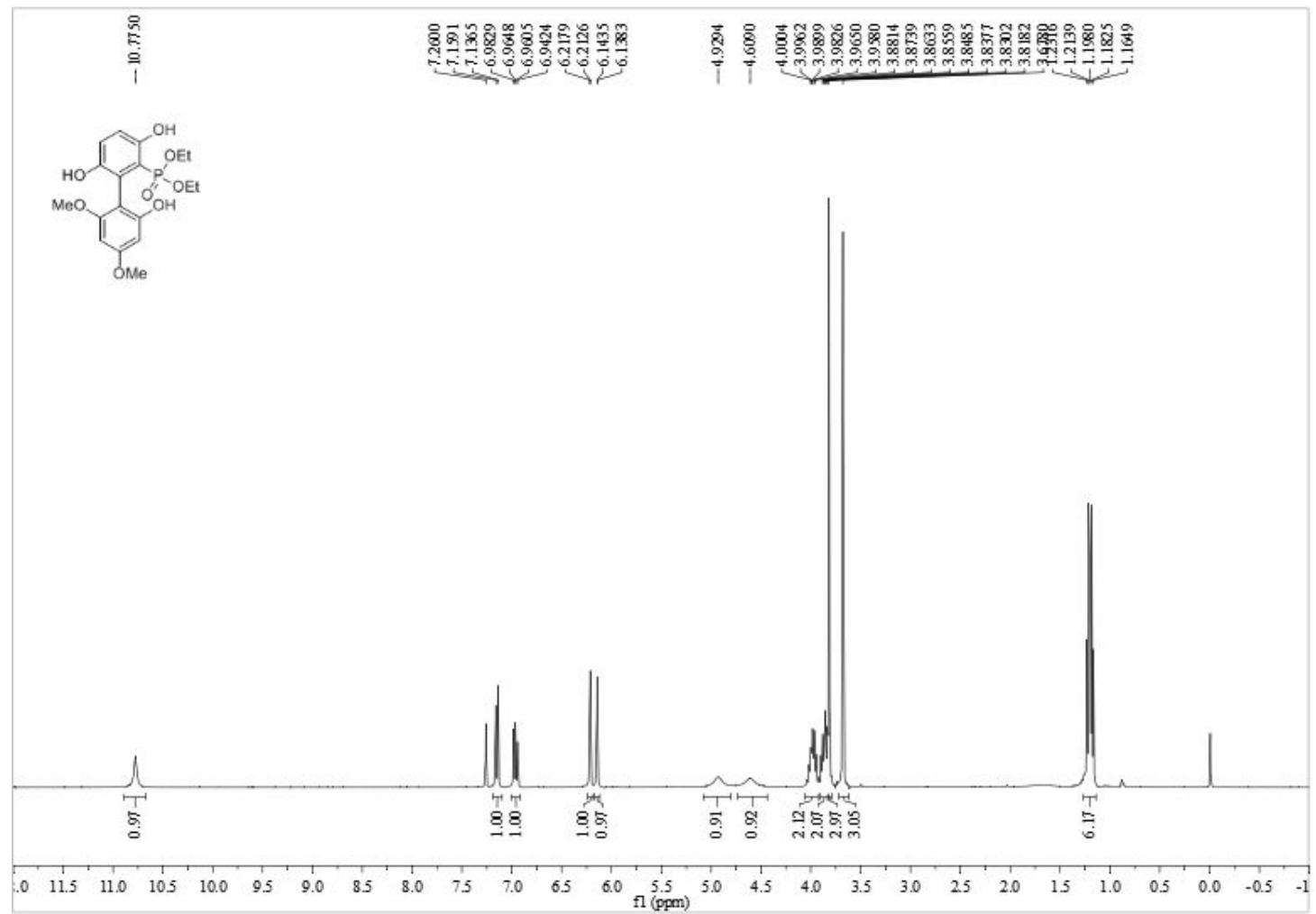

${ }^{31} \mathrm{P}$ NMR for compound $4 \mathbf{c n}\left(162 \mathrm{MHz}, \mathrm{CDCl}_{3}\right)$

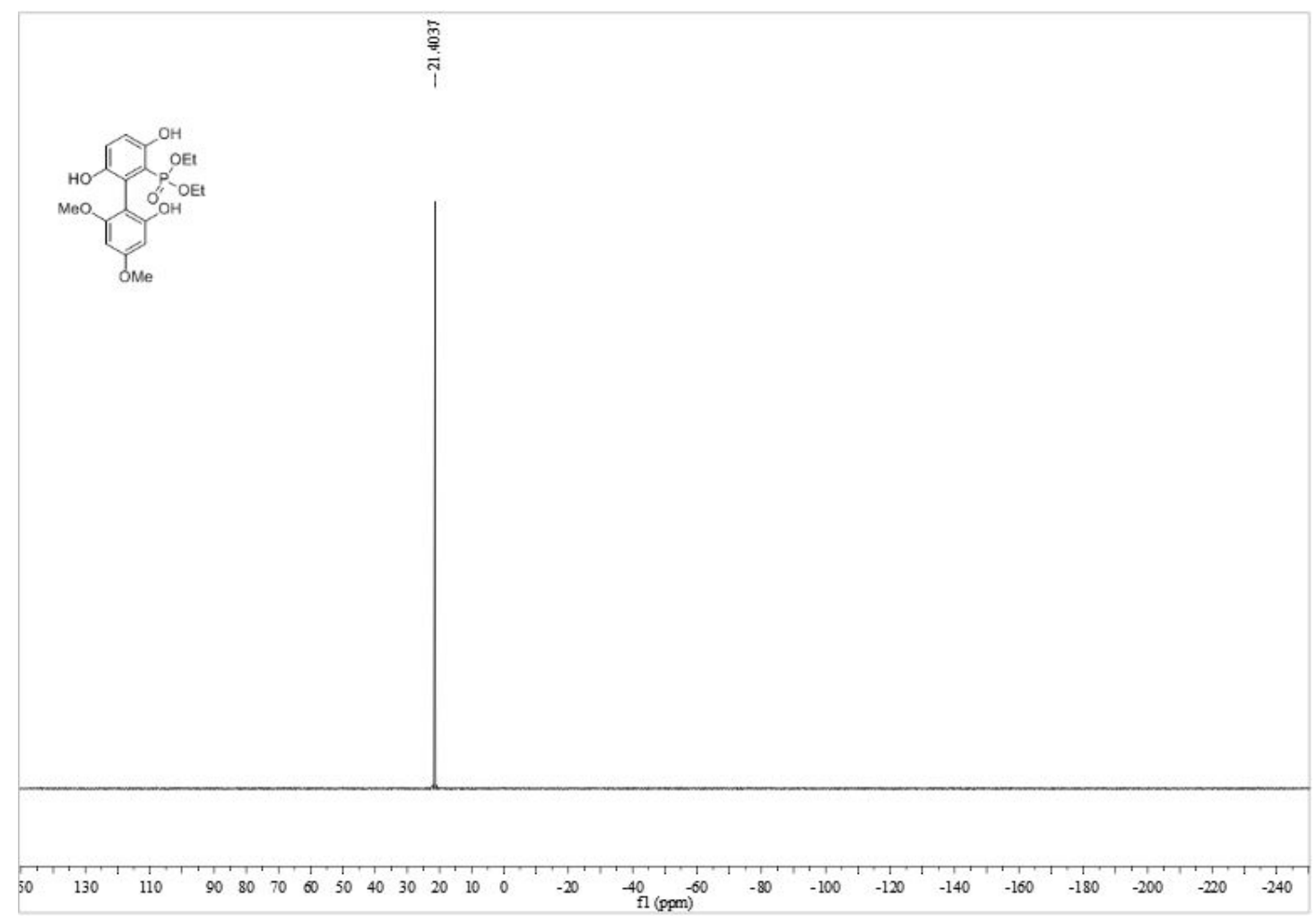


${ }^{13} \mathrm{C}\left\{{ }^{1} \mathrm{H}\right\} \mathrm{NMR}$ for compound $4 \mathbf{c n}\left(101 \mathrm{MHz}, \mathrm{CDCl}_{3}\right)$

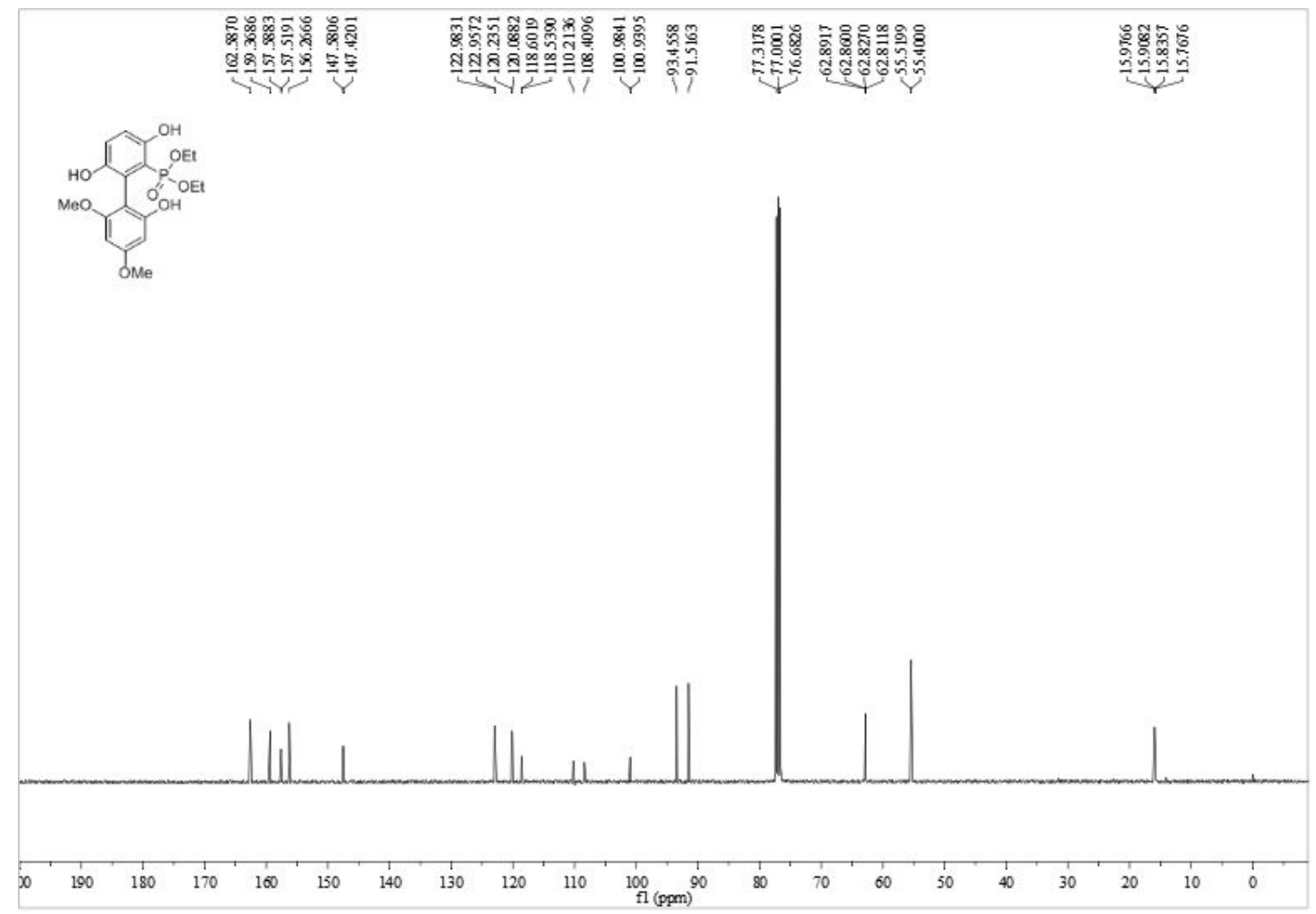

${ }^{1} \mathrm{H}$ NMR for compound 5 (400 MHz, $\mathrm{CDCl}_{3}$ )

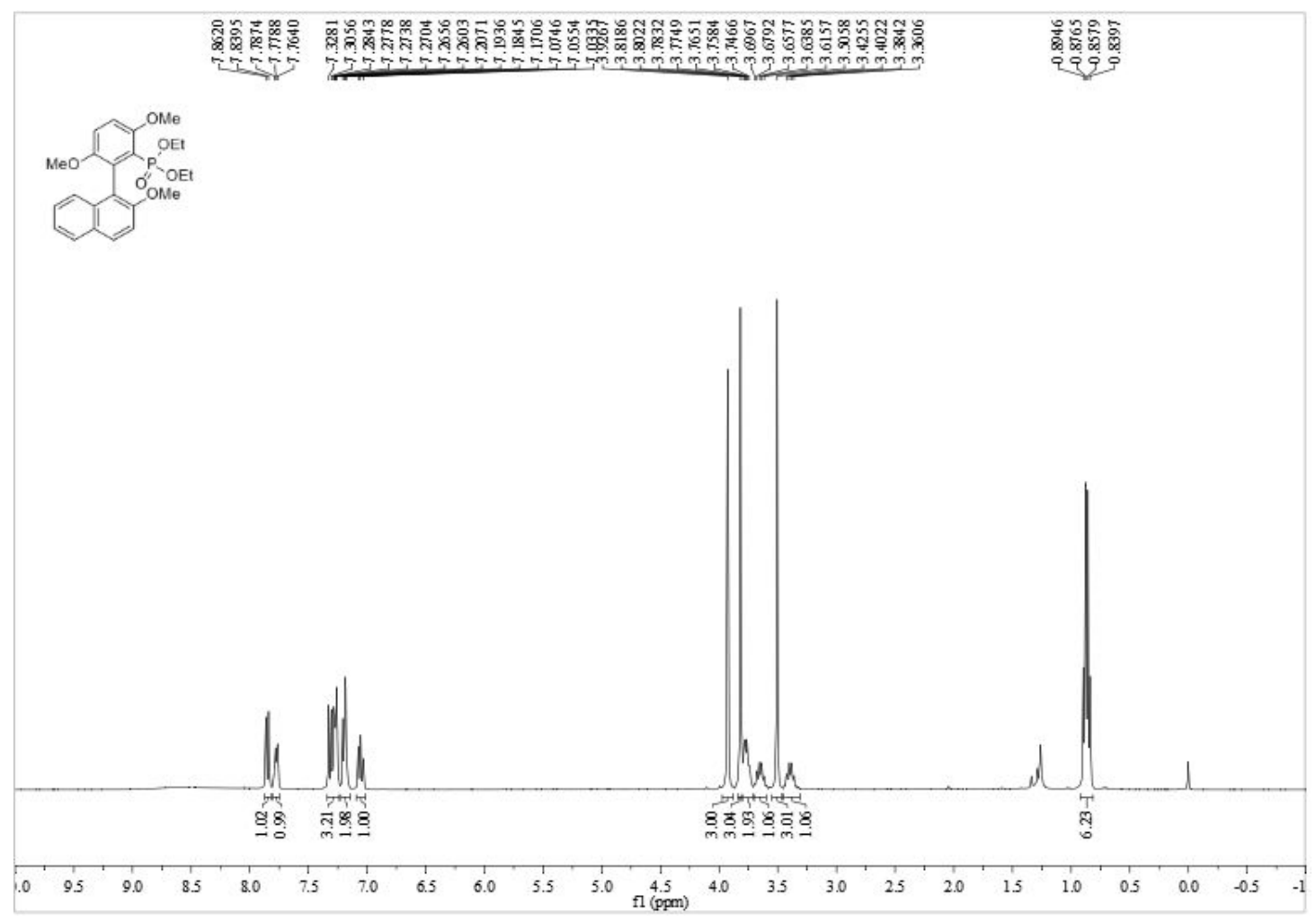


${ }^{31} \mathrm{P}$ NMR for compound $5\left(162 \mathrm{MHz}, \mathrm{CDCl}_{3}\right)$

定
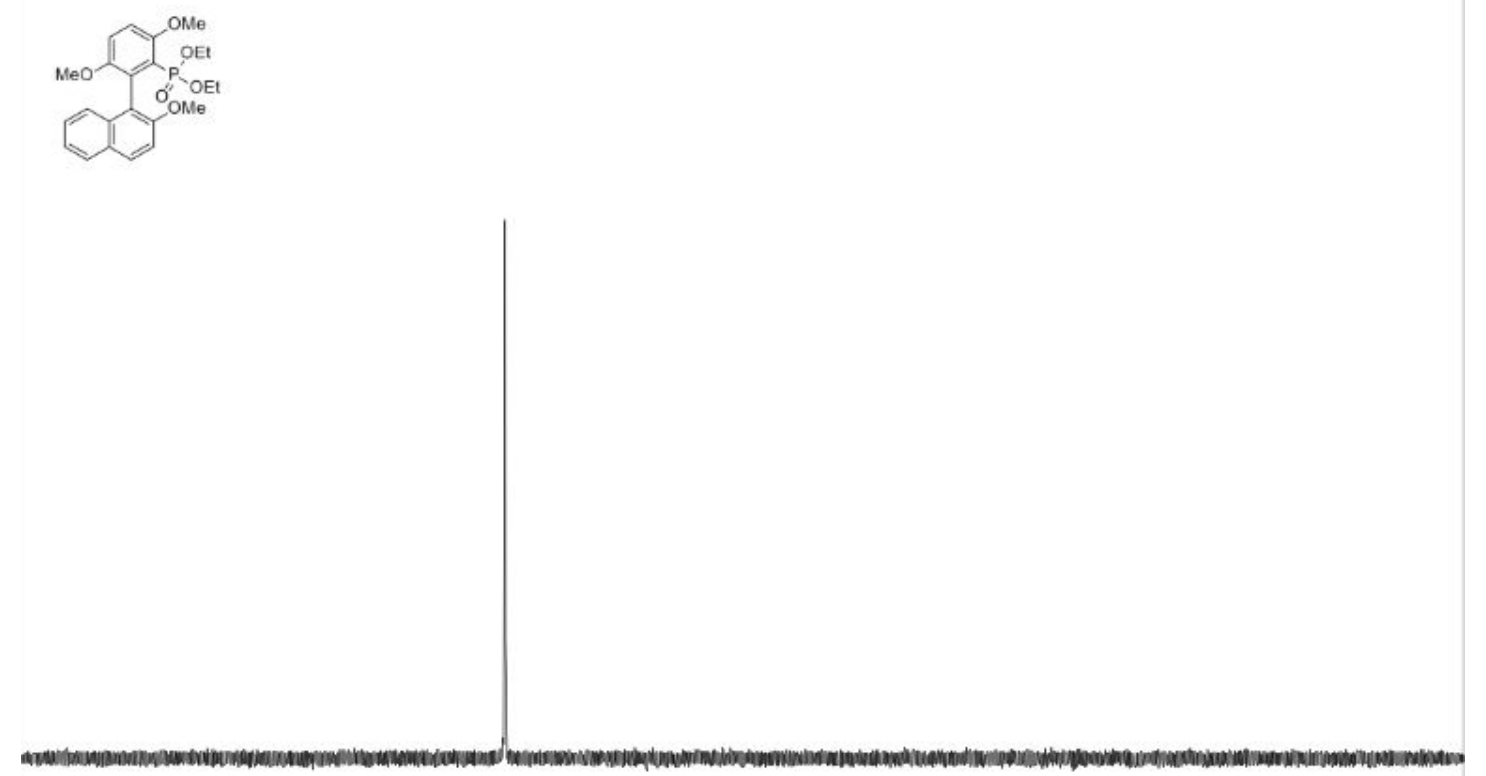

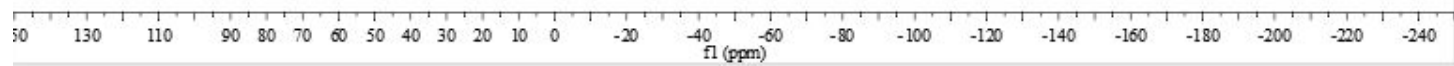

${ }^{13} \mathrm{C}\left\{{ }^{1} \mathrm{H}\right\}$ NMR for compound $5\left(151 \mathrm{MHz}, \mathrm{CDCl}_{3}\right)$

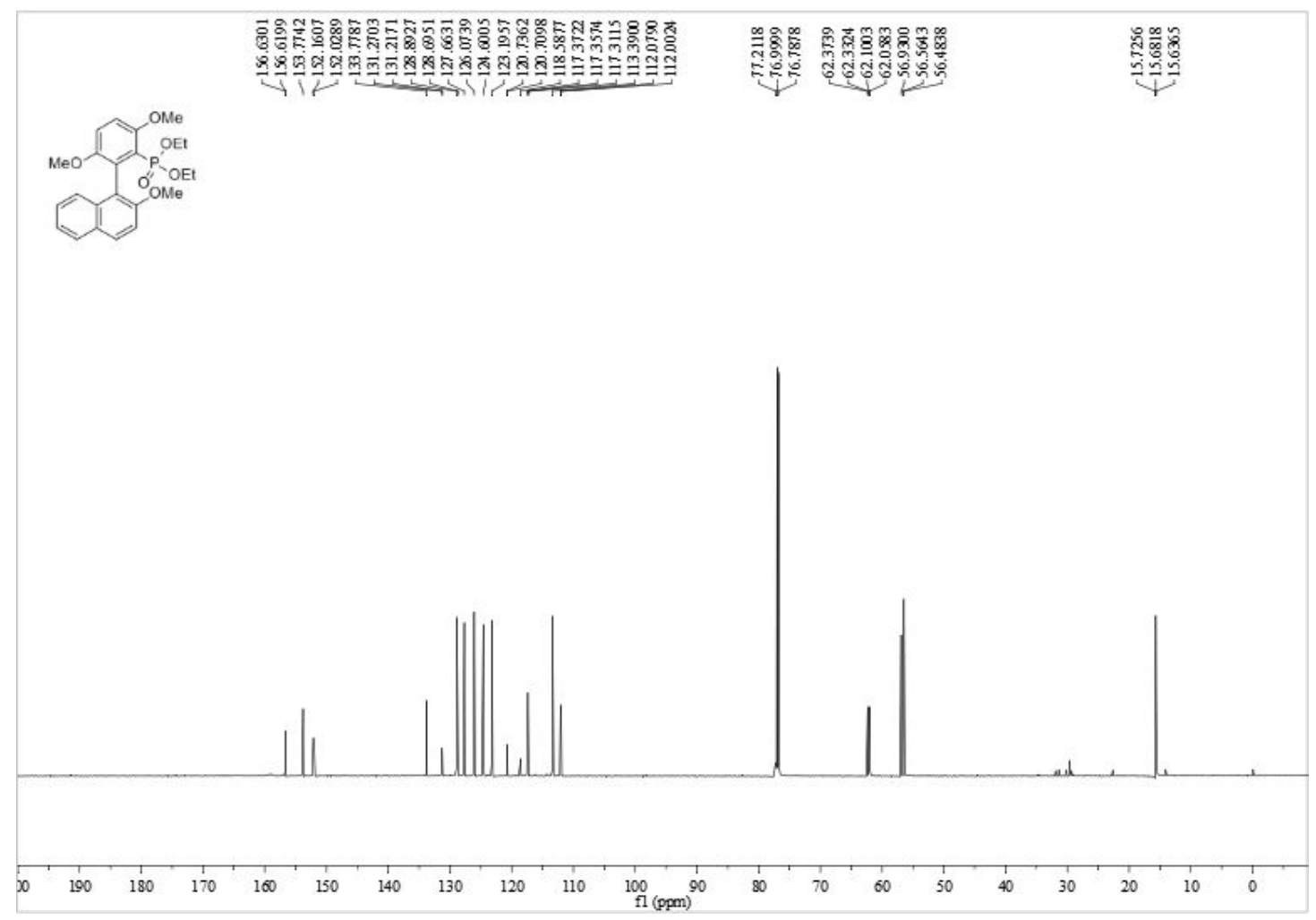


${ }^{1} \mathrm{H}$ NMR for compound $6\left(400 \mathrm{MHz}, \mathrm{CDCl}_{3}\right.$ )

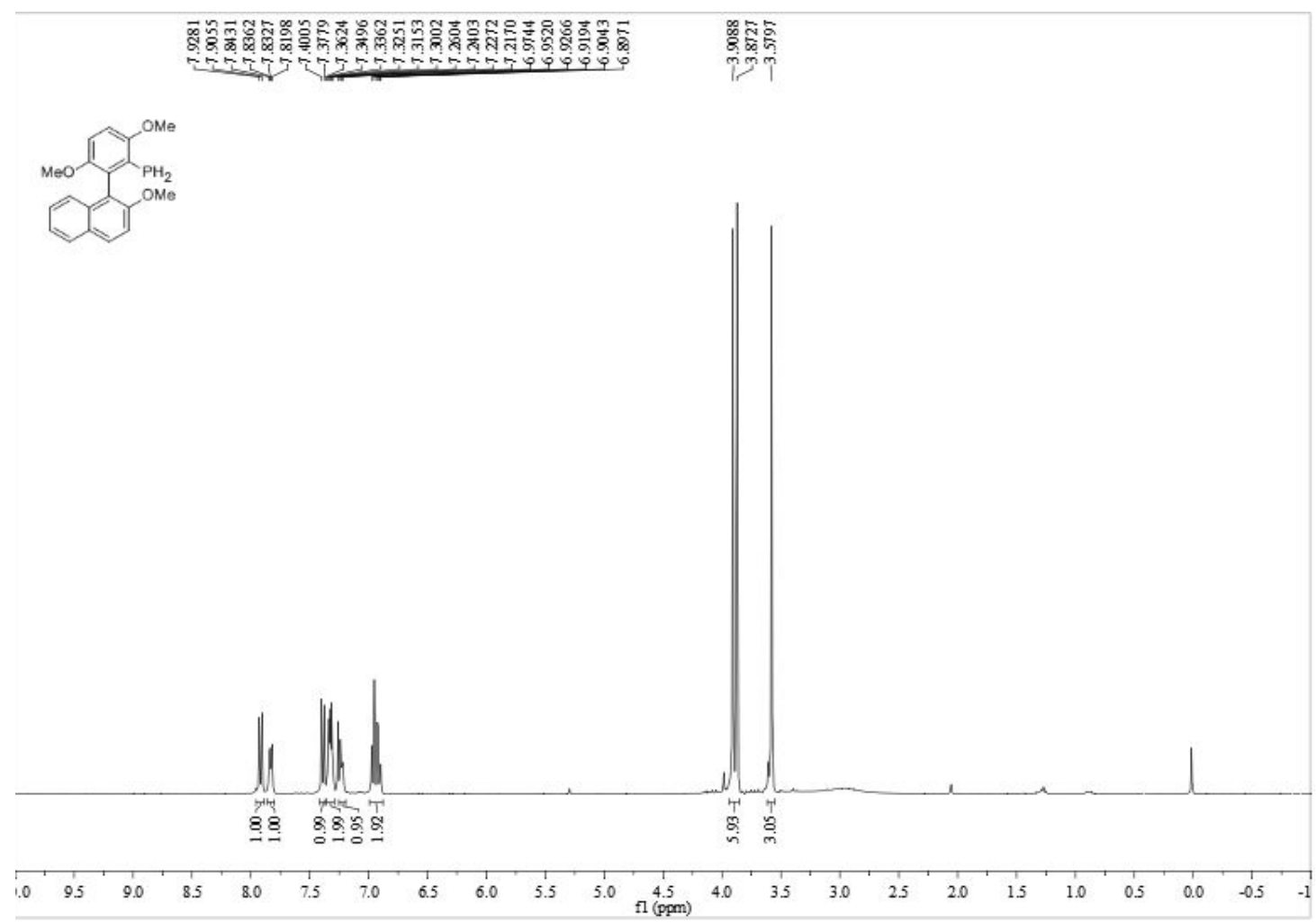

${ }^{31} \mathrm{P}$ NMR for compound $6\left(162 \mathrm{MHz}, \mathrm{CDCl}_{3}\right)$

\section{$\stackrel{n}{\frac{\infty}{\infty}}$}
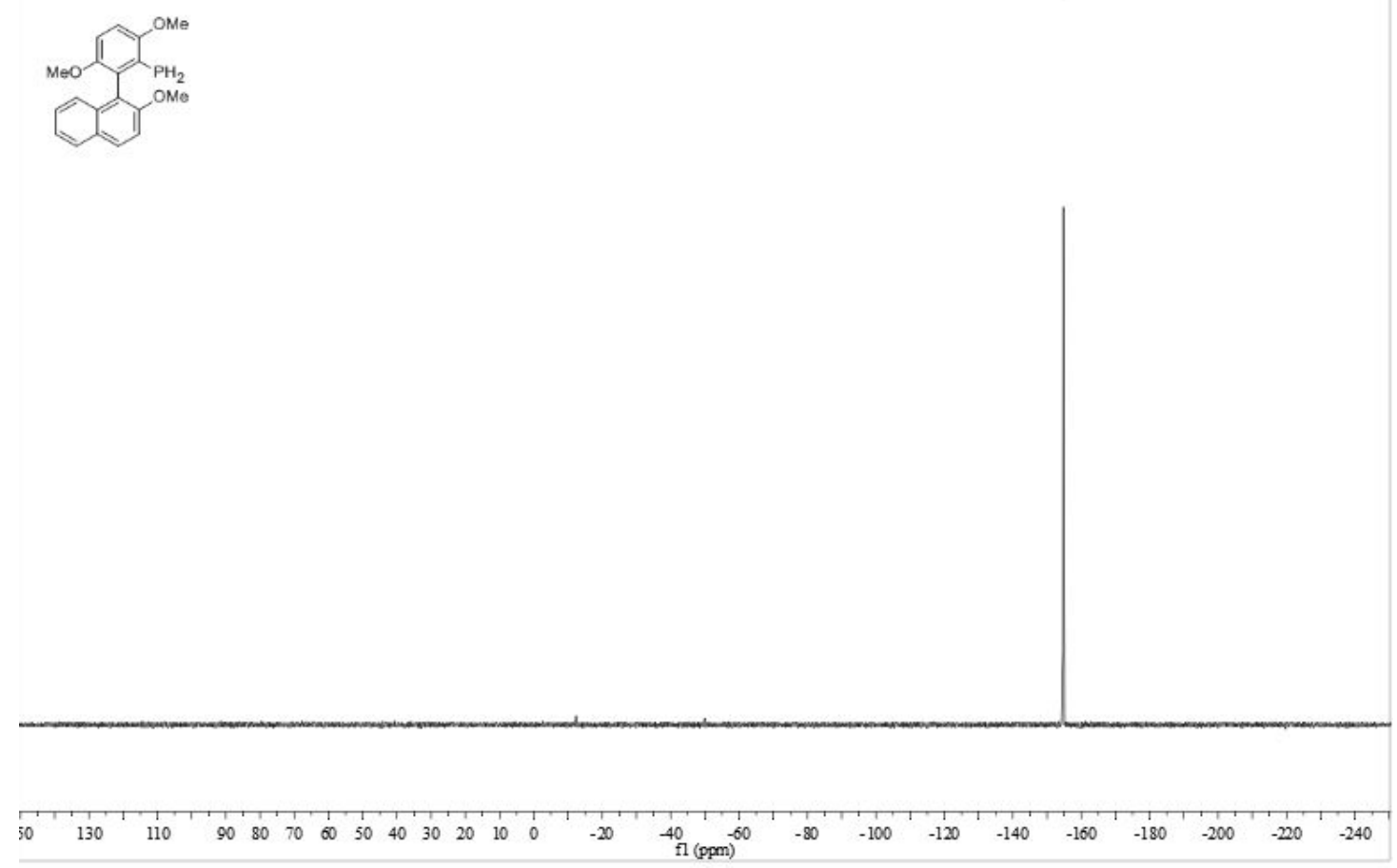
${ }^{13} \mathrm{C}\left\{{ }^{1} \mathrm{H}\right\}$ NMR for compound $6\left(151 \mathrm{MHz}, \mathrm{CDCl}_{3}\right)$

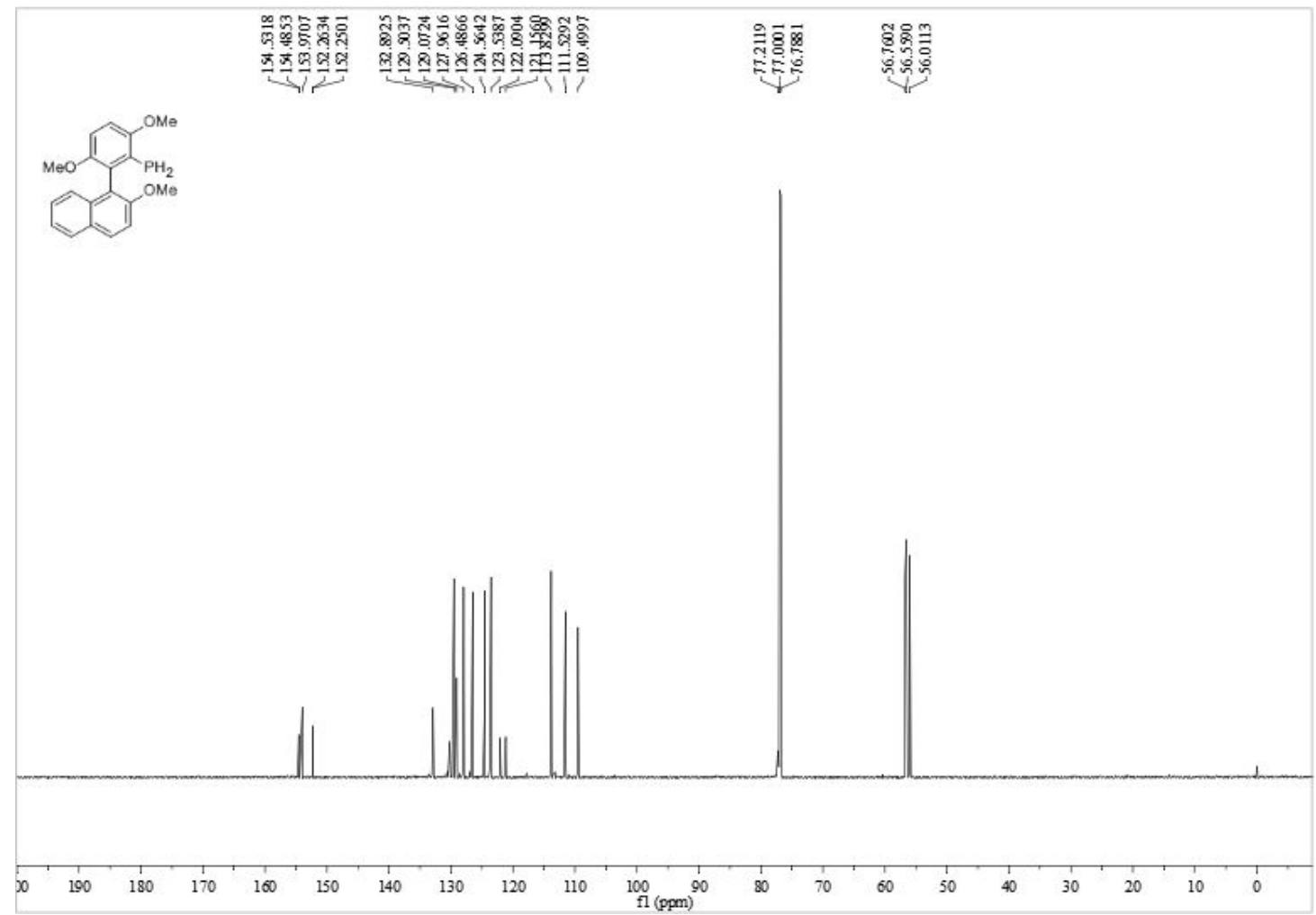

${ }^{1} \mathrm{H}$ NMR for compound $7 \mathbf{a}\left(400 \mathrm{MHz}, \mathrm{CDCl}_{3}\right)$

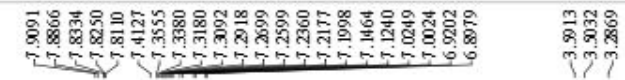

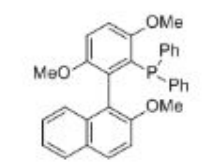


${ }^{31} \mathrm{P}$ NMR for compound $7 \mathbf{a}\left(162 \mathrm{MHz}, \mathrm{CDCl}_{3}\right)$

\section{器}
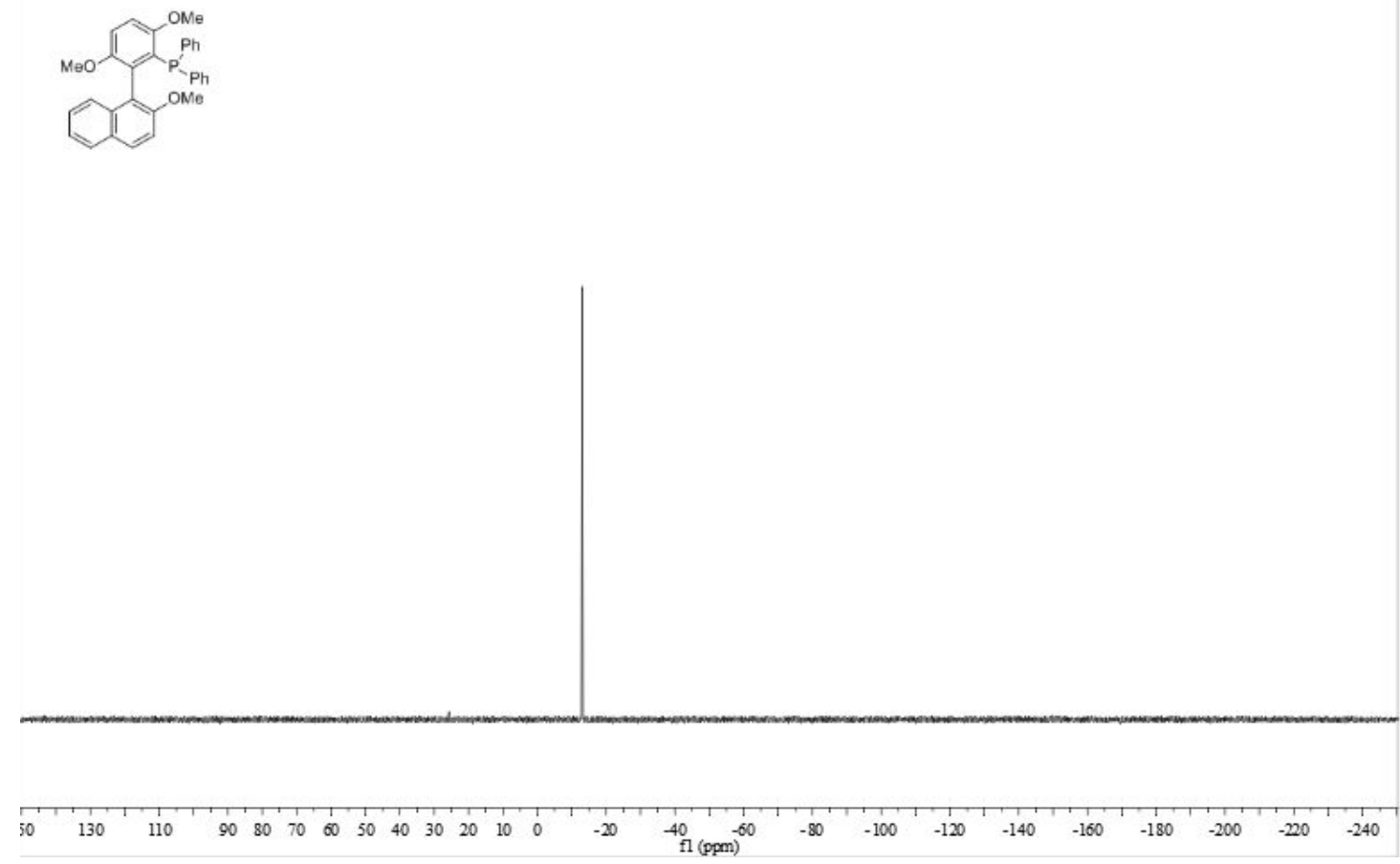

${ }^{13} \mathrm{C}\left\{{ }^{1} \mathrm{H}\right\}$ NMR for compound $7 \mathbf{a}\left(151 \mathrm{MHz}, \mathrm{CDCl}_{3}\right)$

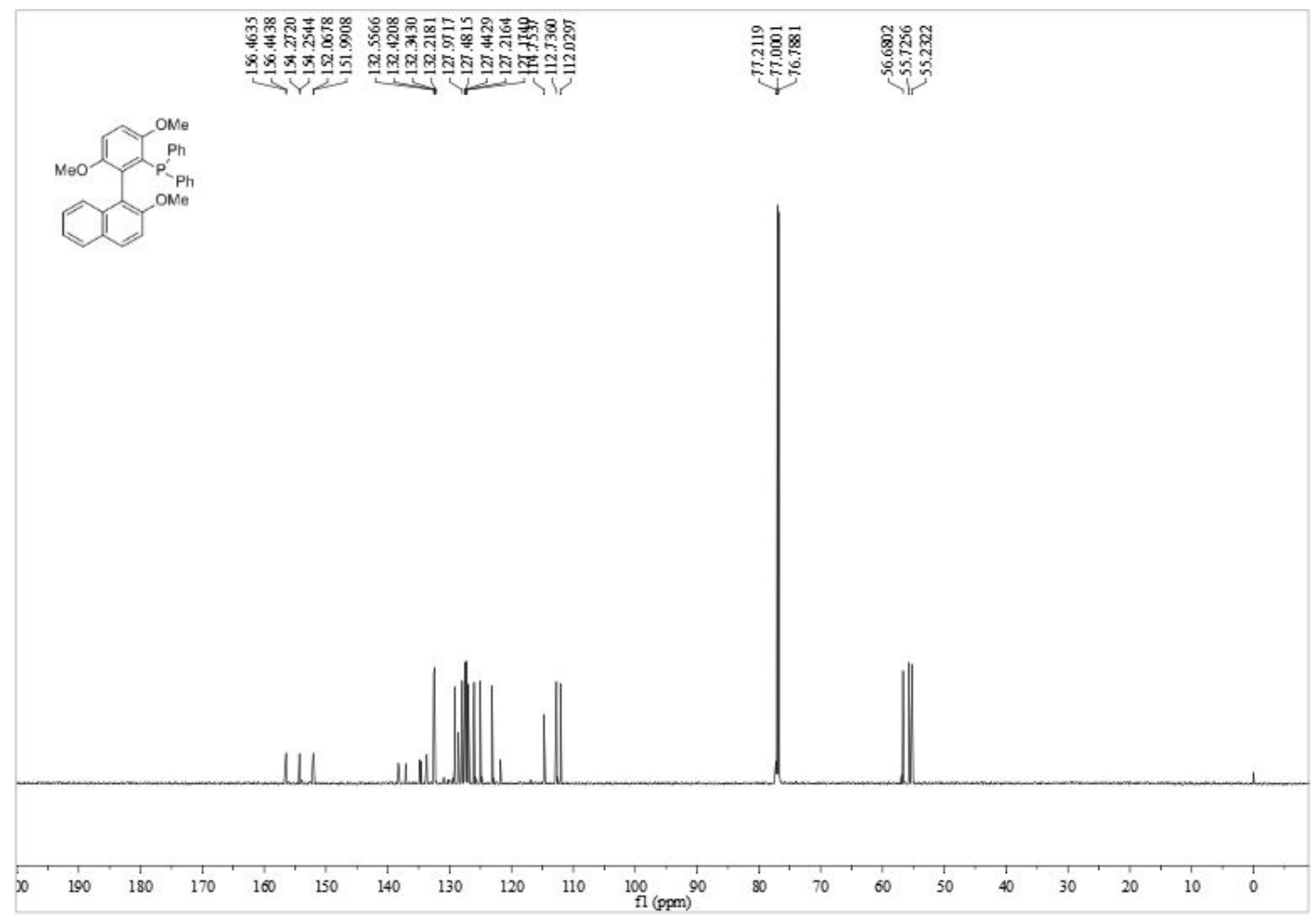


${ }^{1} \mathrm{H}$ NMR for compound $7 \mathbf{b}\left(400 \mathrm{MHz}, \mathrm{CDCl}_{3}\right)$

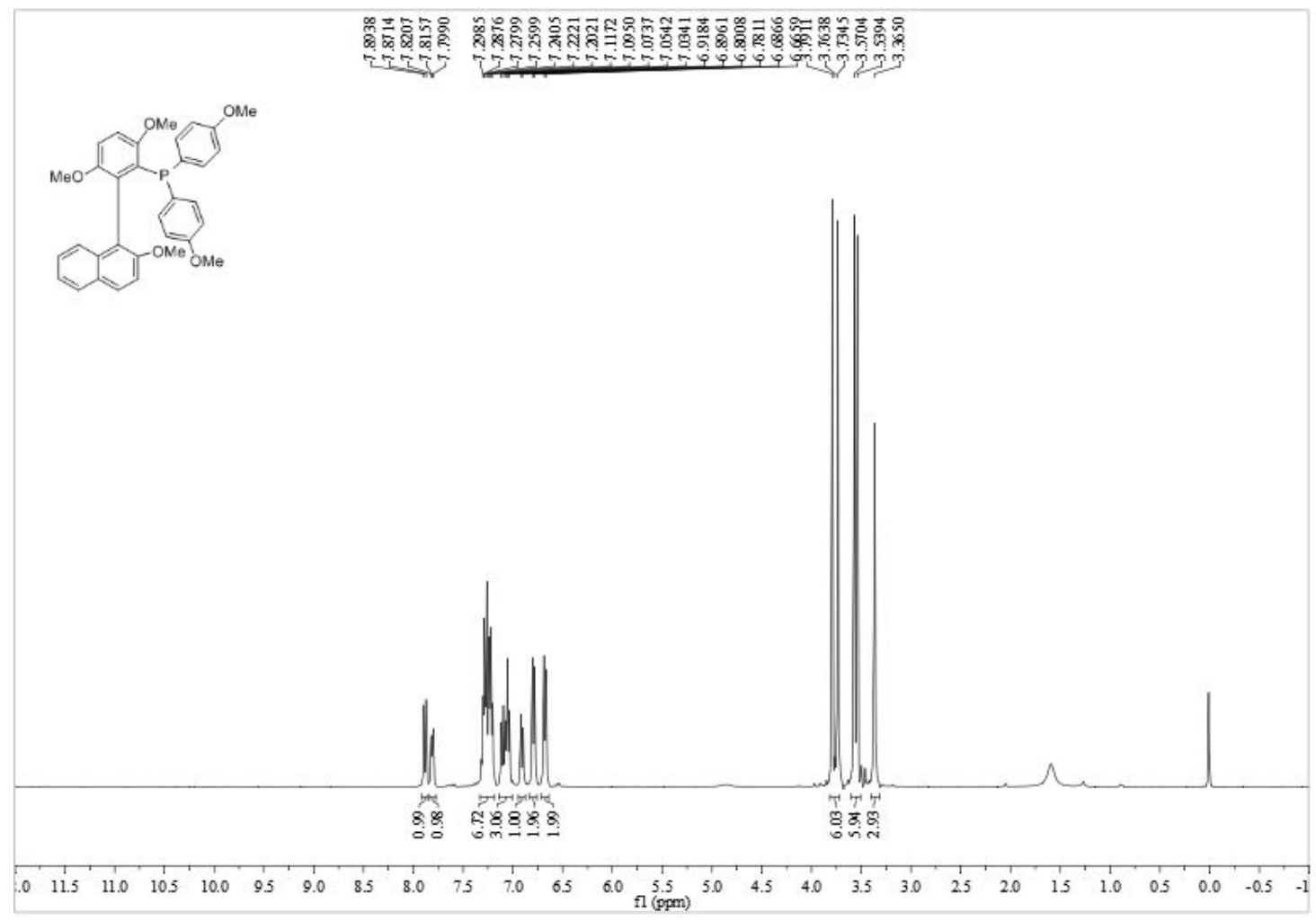

${ }^{31} \mathrm{P}$ NMR for compound $7 \mathbf{b}\left(162 \mathrm{MHz}, \mathrm{CDCl}_{3}\right)$

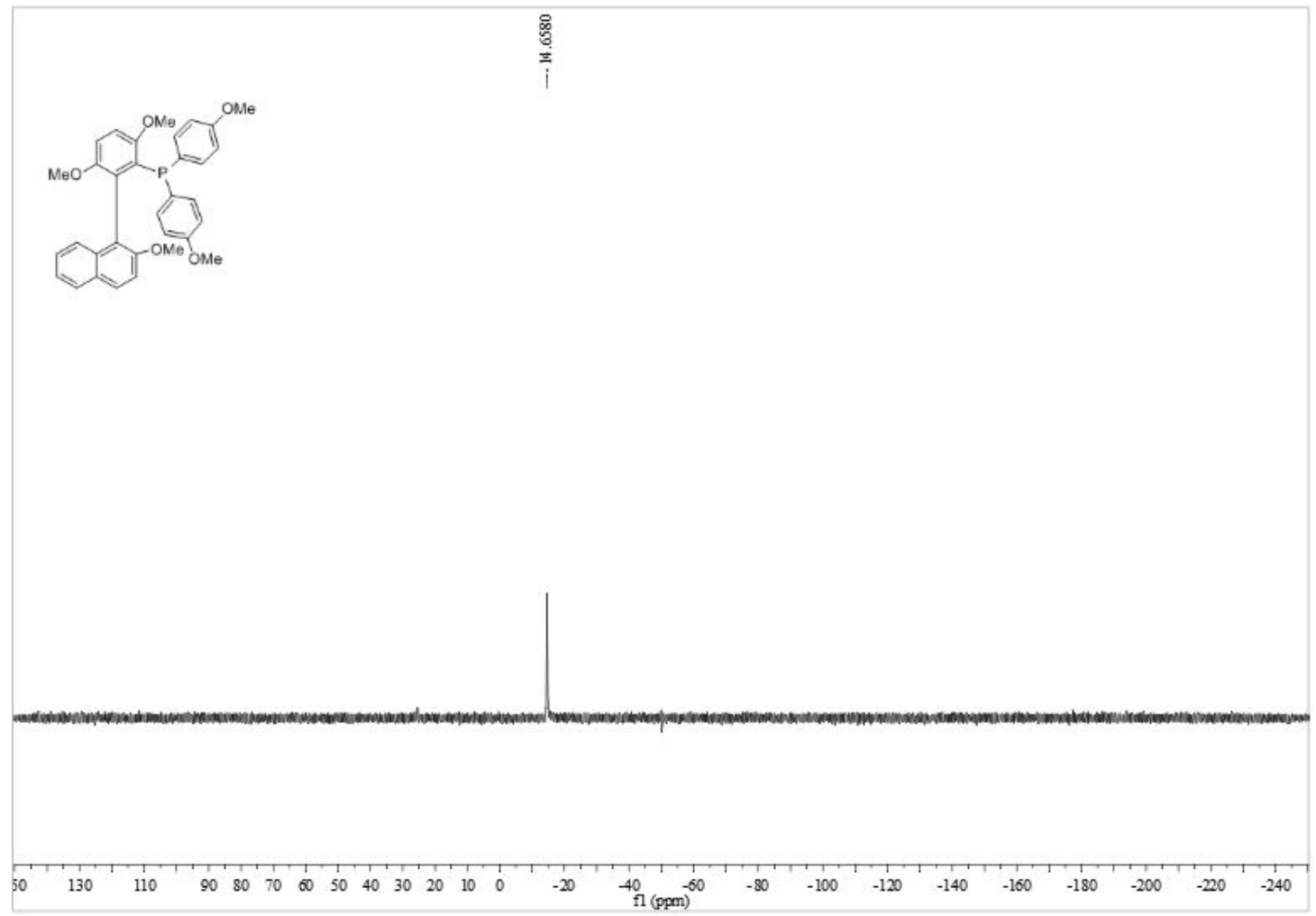


${ }^{13} \mathrm{C}\left\{{ }^{1} \mathrm{H}\right\}$ NMR for compound $\mathbf{7 b}\left(151 \mathrm{MHz}, \mathrm{CDCl}_{3}\right)$
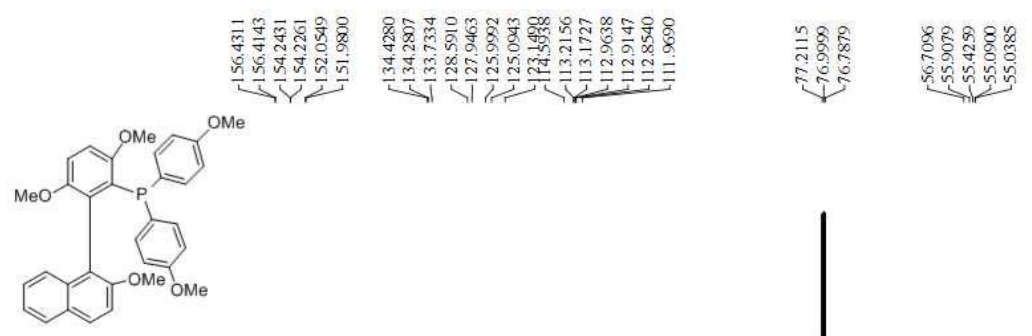

U

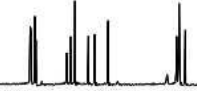

$10 \quad 190$

$\begin{array}{lllllllll}180 & 170 & 160 & 150 & 140 & 130 & 120 & 110 & \begin{array}{l}100 \\ \mathrm{fl}(\mathrm{ppm})\end{array}\end{array}$

${ }^{1} \mathrm{H}$ NMR for compound $8\left(400 \mathrm{MHz}, \mathrm{CDCl}_{3}\right)$

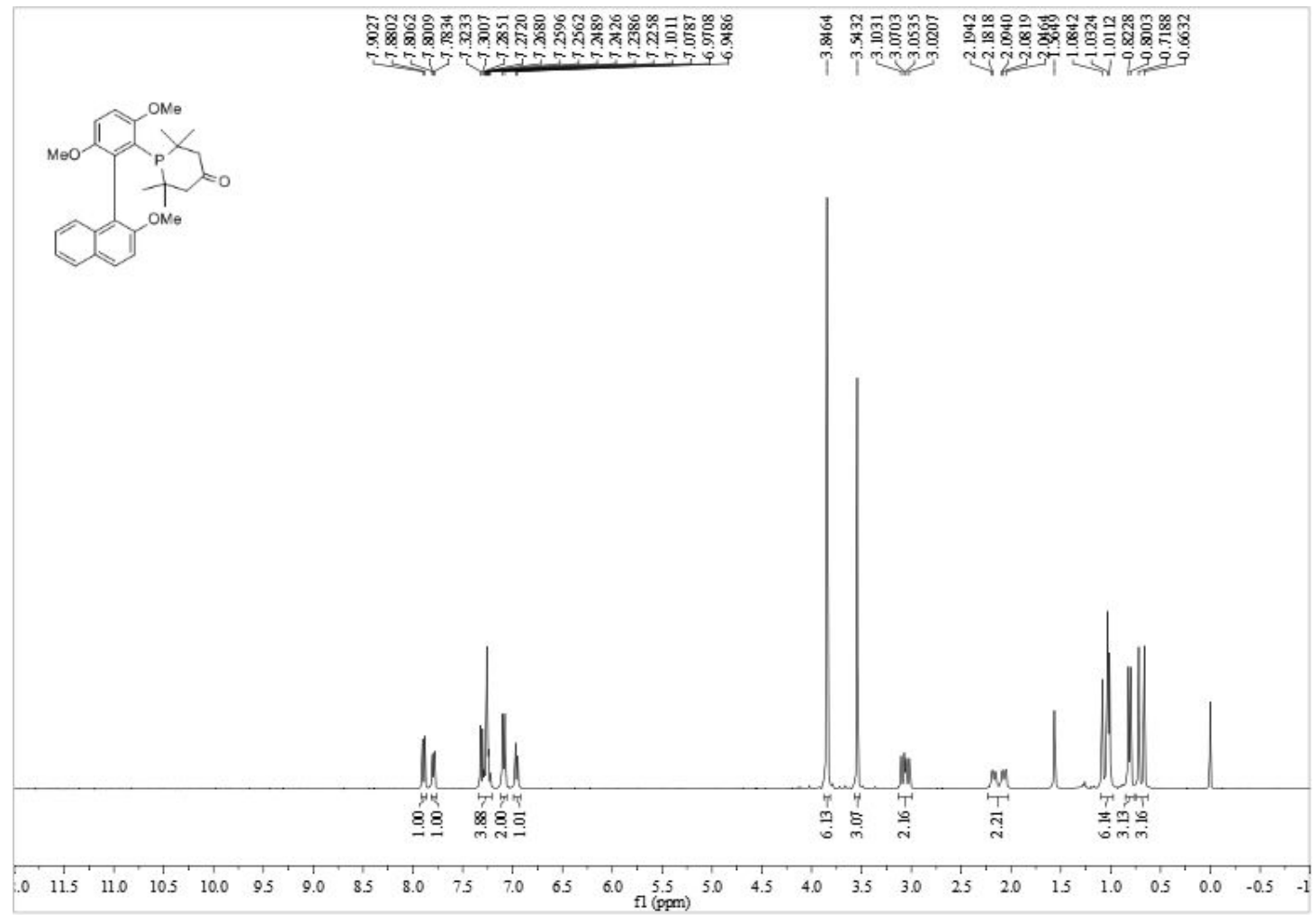


${ }^{31} \mathrm{P}$ NMR for compound $8\left(162 \mathrm{MHz}, \mathrm{CDCl}_{3}\right)$

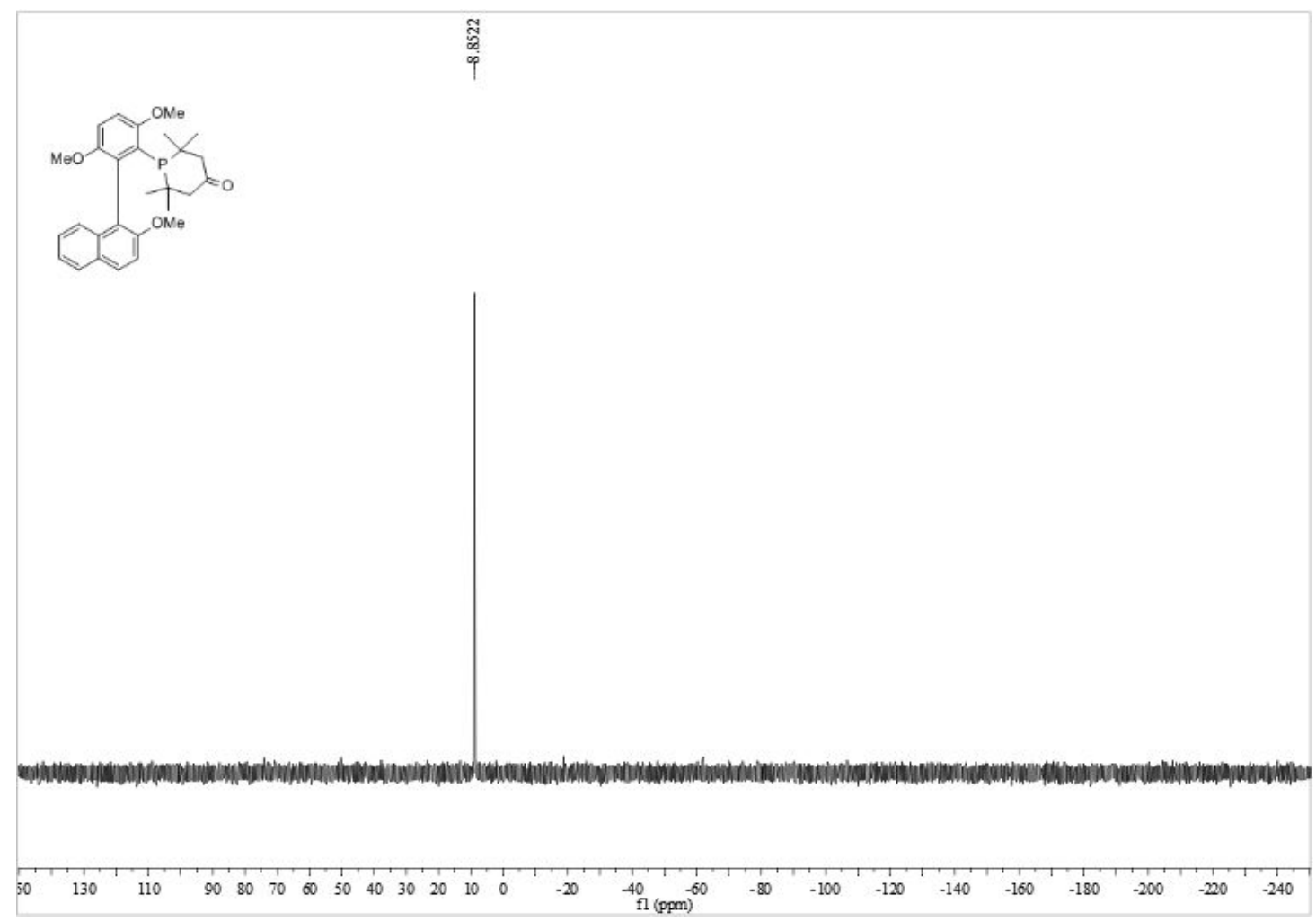

${ }^{13} \mathrm{C}\left\{{ }^{1} \mathrm{H}\right\}$ NMR for compound $9\left(151 \mathrm{MHz}, \mathrm{CDCl}_{3}\right)$

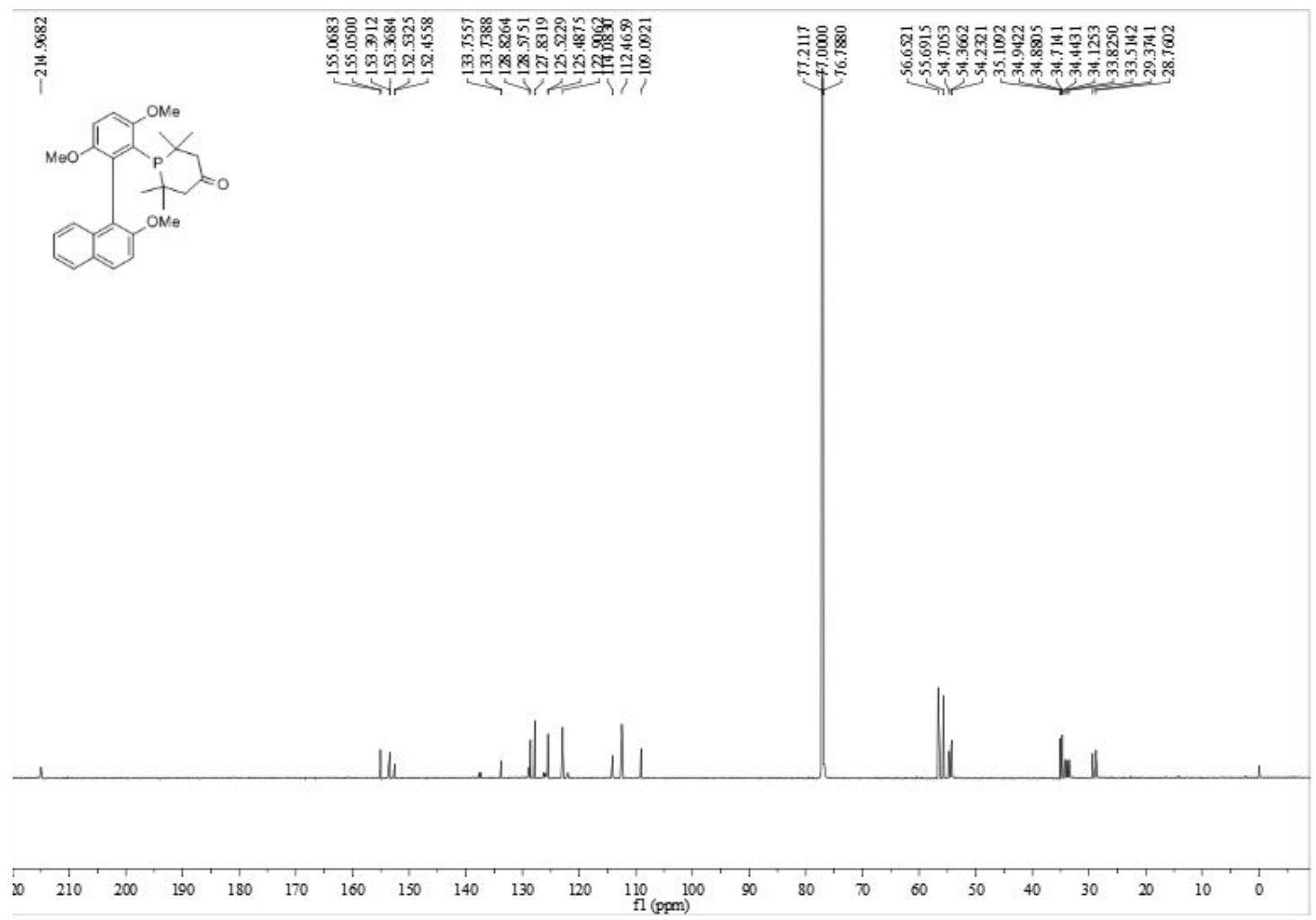


${ }^{1} \mathrm{H}$ NMR for compound $9\left(400 \mathrm{MHz}, \mathrm{CDCl}_{3}\right)$

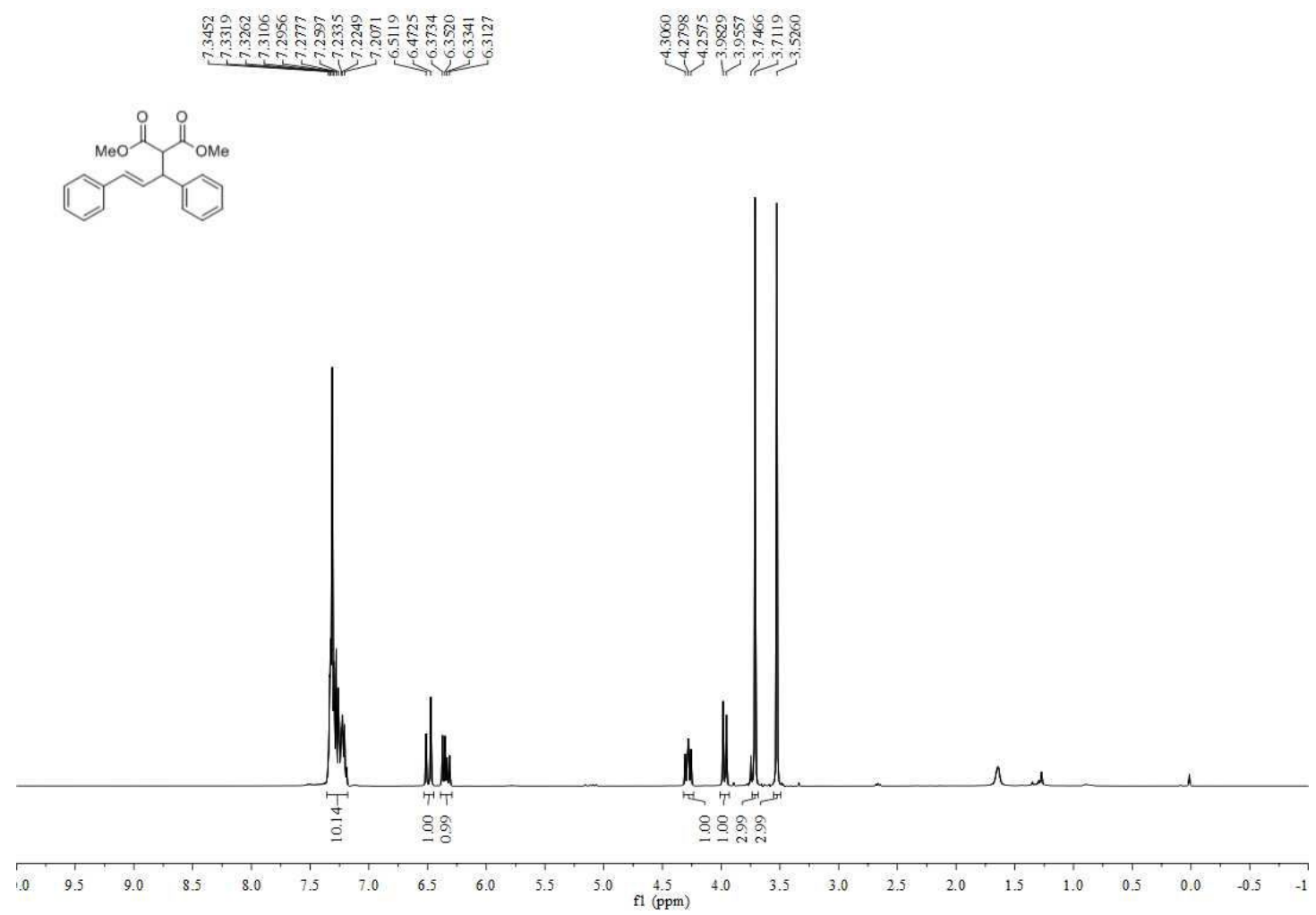

${ }^{13} \mathrm{C}\left\{{ }^{1} \mathrm{H}\right\}$ NMR for compound $9\left(101 \mathrm{MHz}, \mathrm{CDCl}_{3}\right)$
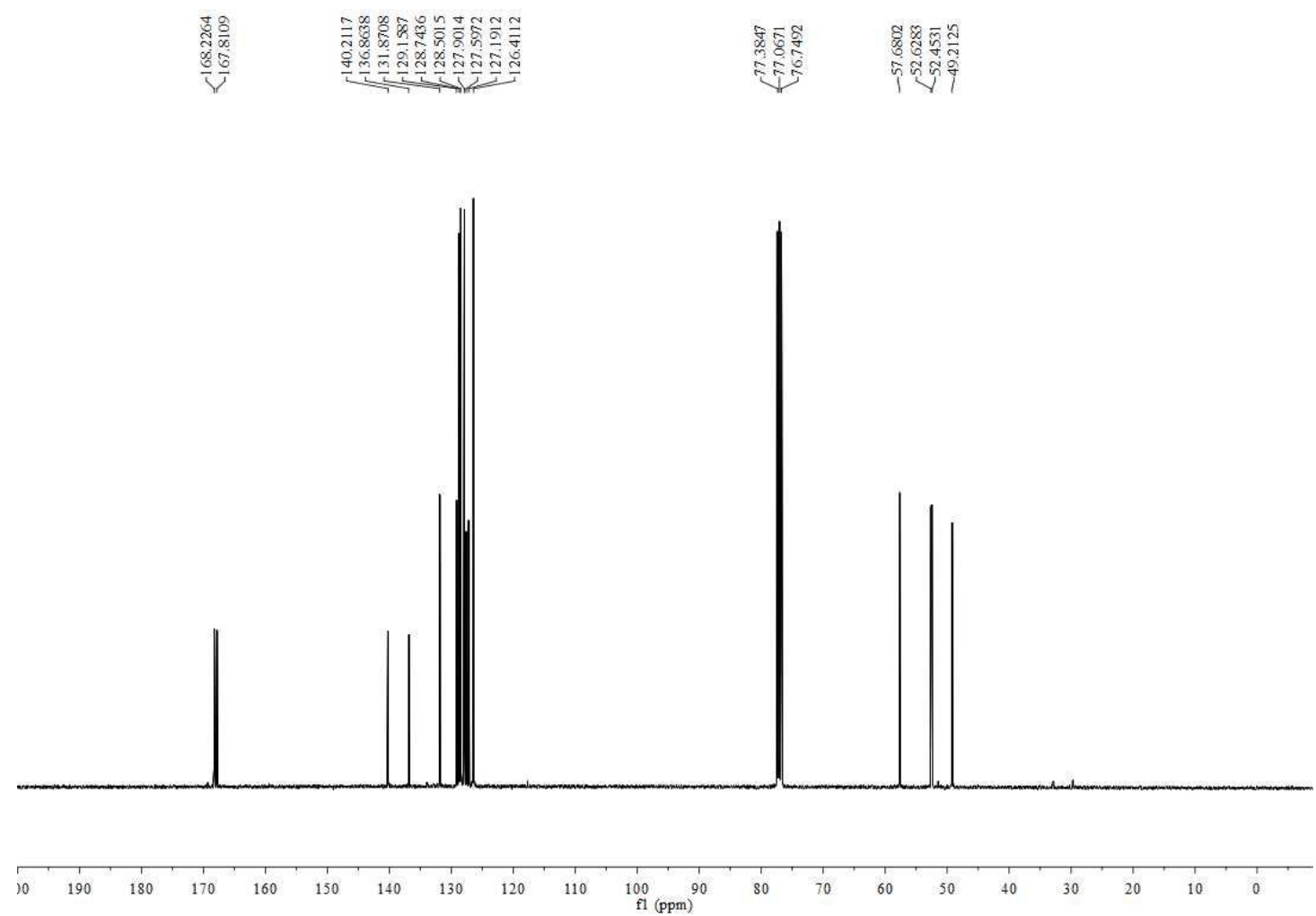


\section{5、HPLC spectra}

\section{HPLC spectra of Rac-4aa}

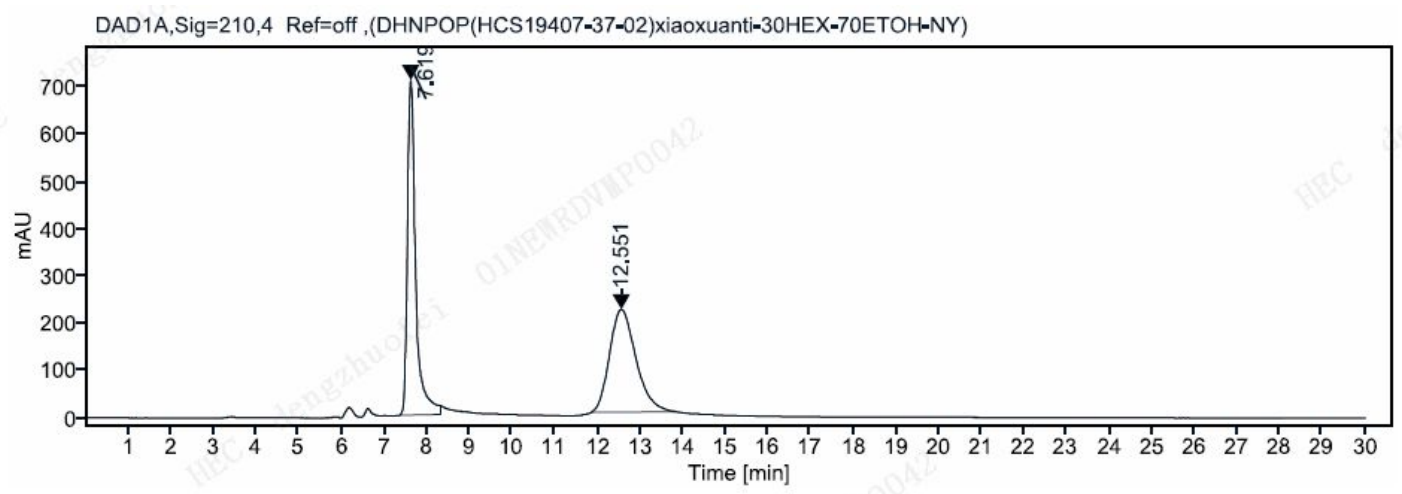

Signal: $\quad$ DAD1A,Sig=210,4 Ref=off

$\begin{array}{ccccccc}\text { RT [min] } & \text { Height } & \text { Area } & \text { Area \% } & \begin{array}{c}\text { Resolution } \\ \text { USP }\end{array} & \text { Tail } & \begin{array}{c}\text { Peak Theoretical } \\ \text { Plates USP }\end{array} \\ 7.62 & 709.579 & 9503.217 & 50.38 & & 1.7 & 9556.12487 \\ 12.55 & 217.524 & 9359.442 & 49.62 & 6.8 & 1.3 & 1968.72331\end{array}$

HPLC spectra of (R)-4aa

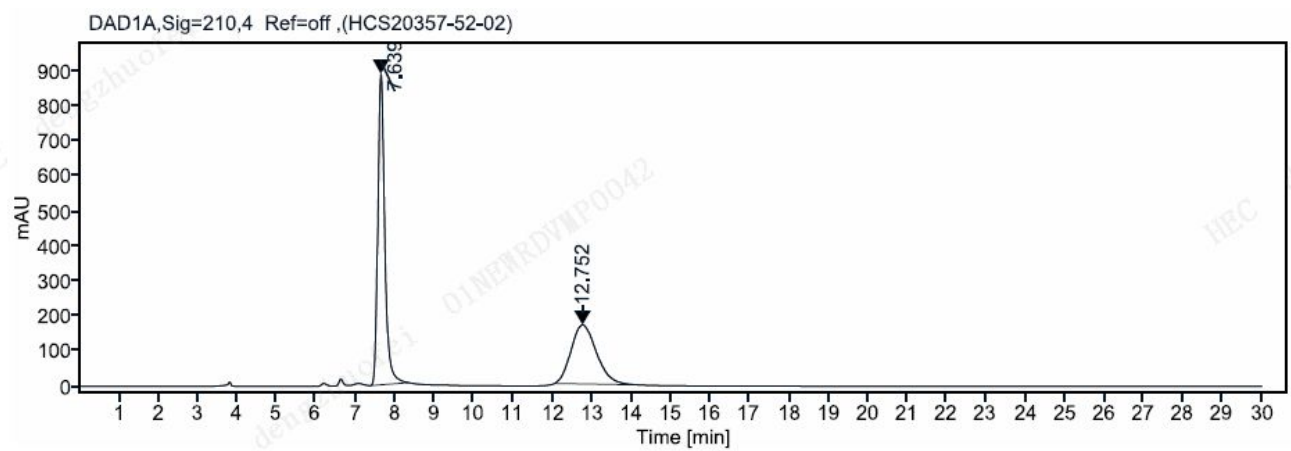

Signal: $\quad$ DAD1A,Sig $=210,4$ Ref $=$ off

$\begin{array}{ccccccc}\text { RT [min] } & \text { Height } & \text { Area } & \text { Area \% } & \begin{array}{c}\text { Resolution } \\ \text { USP }\end{array} & \text { Tail } & \begin{array}{c}\text { Theoretical } \\ \text { Plates USP }\end{array} \\ 7.64 & 887.932 & 10856.577 & 58.81 & & 1.3 & 9836 \\ 12.75 & 168.998 & 7603.596 & 41.19 & 6.8 & 1.2 & 1817\end{array}$


HPLC spectra of Rac-4ba

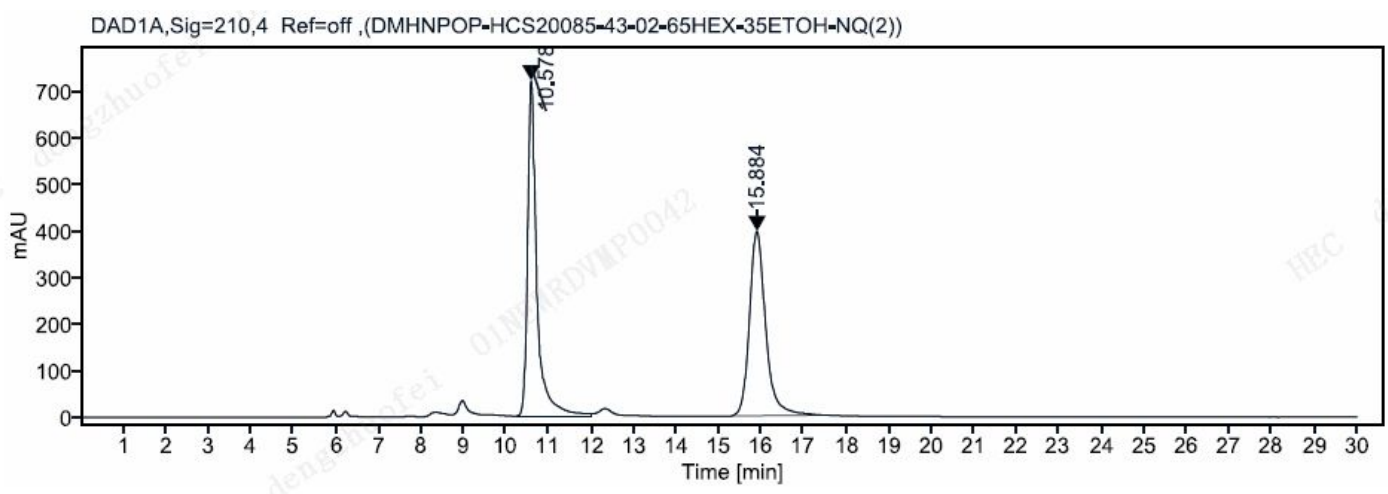

Signal: DAD1A,Sig=210,4 Ref=off

$\begin{array}{ccccccc}\text { RT [min] } & \text { Height } & \text { Area } & \text { Area \% } & \begin{array}{c}\text { Resolution } \\ \text { USP }\end{array} & \text { Tail } & \begin{array}{c}\text { Peak Theoretical } \\ \text { Plates USP }\end{array} \\ 10.58 & 719.833 & 10904.348 & 51.07 & & 1.6 & 15564.13736 \\ 15.88 & 395.543 & 10448.439 & 48.93 & 10.6 & 1.3 & 9249.63357\end{array}$

HPLC spectra of (R)-4ba

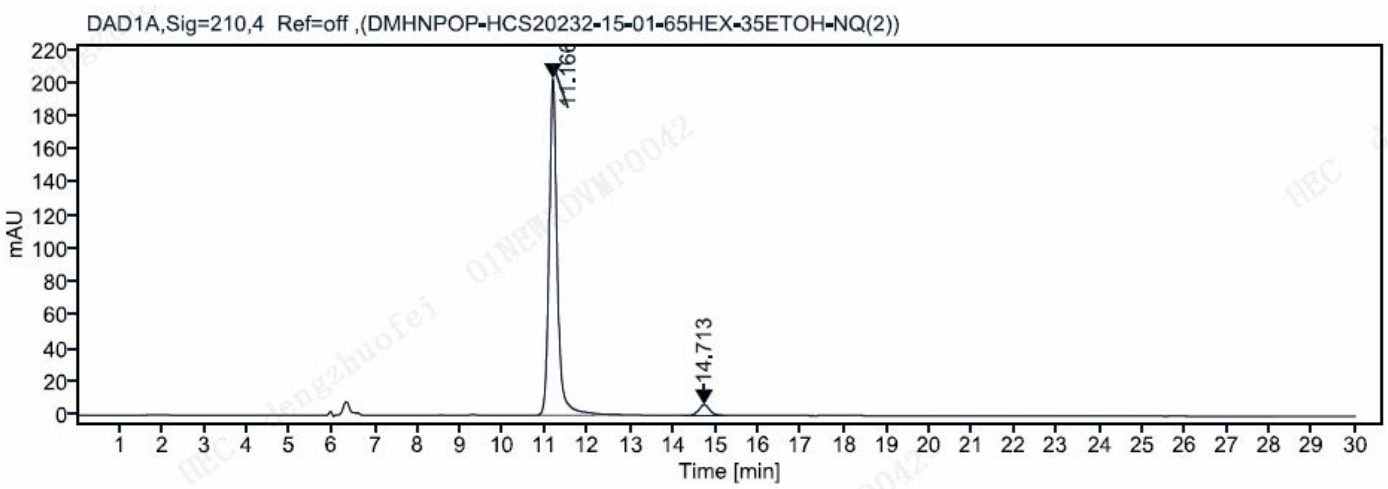

Signal: $\quad$ DAD1A,Sig $=210,4$ Ref $=$ off

$\begin{array}{ccccccc}\text { RT [min] } & \text { Height } & \text { Area } & \text { Area \% } & \begin{array}{c}\text { Resolution } \\ \text { USP }\end{array} & \text { Tail } & \begin{array}{c}\text { Peak Theoretical } \\ \text { Plates USP }\end{array} \\ 11.17 & 202.739 & 2714.250 & 96.00 & & 1.2 & 18864.45173 \\ 14.71 & 6.356 & 113.237 & 4.00 & 9.0 & 1.1 & 15949.95620\end{array}$


HPLC spectra of Rac-4ca

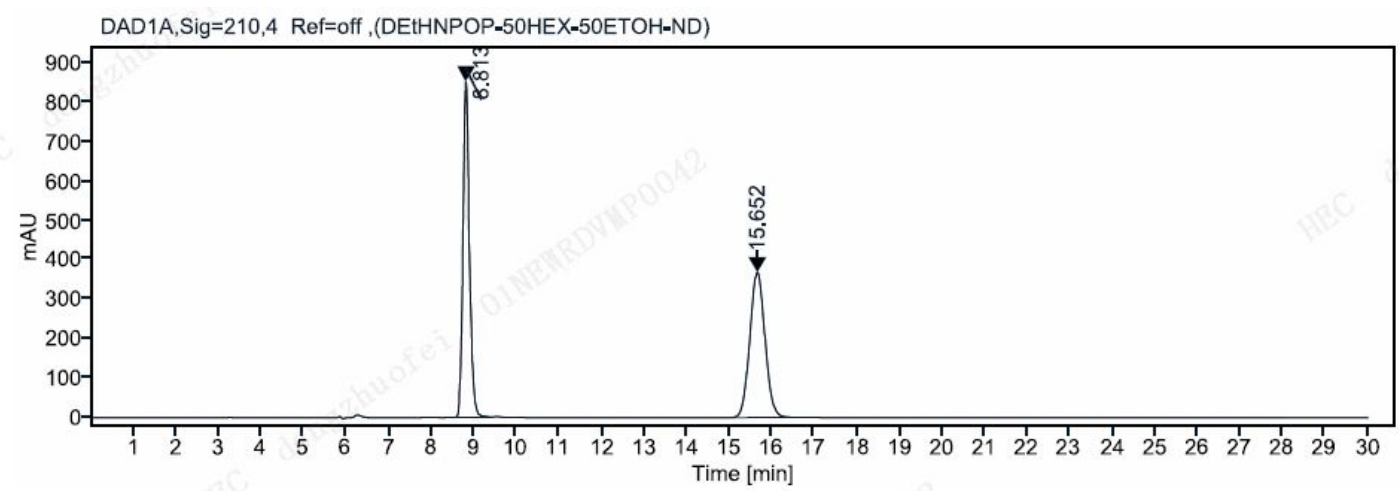

Signal: $\quad$ DAD1A,Sig=210,4 Ref=off

$\begin{array}{ccccccc}\text { RT [min] } & \text { Height } & \text { Area } & \text { Area \% } & \begin{array}{c}\text { Resolution } \\ \text { USP }\end{array} & \text { Tail } & \begin{array}{c}\text { Peak Theoretical } \\ \text { Plates USP }\end{array} \\ 8.81 & 849.803 & 9118.081 & 49.99 & & 1.2 & 16133.89974 \\ 15.65 & 367.171 & 9121.375 & 50.01 & 14.6 & 1.1 & 9057.52055\end{array}$

HPLC spectra of (R)-4ca

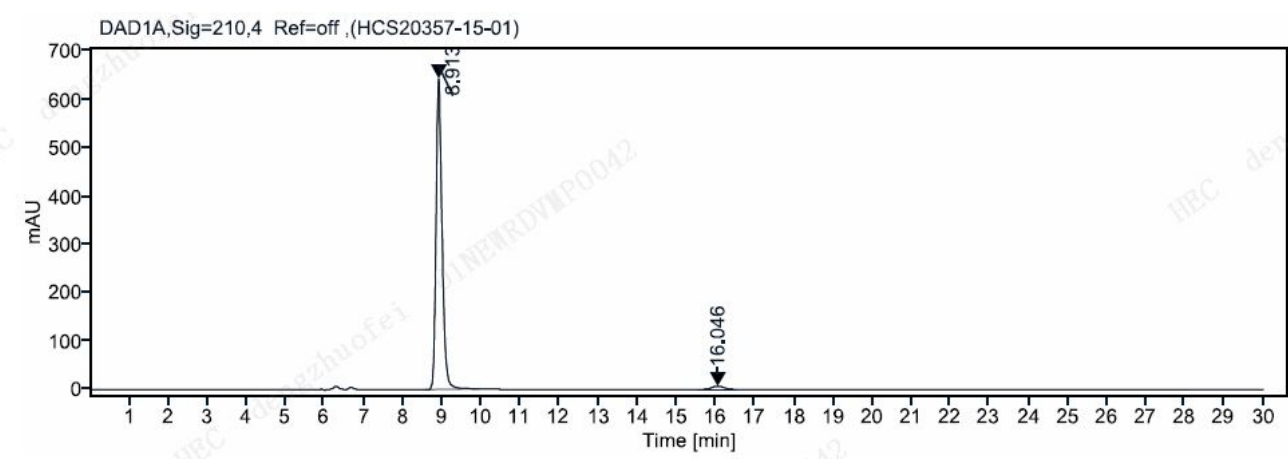

Signal: DAD1A,Sig=210,4 Ref=off

$\begin{array}{ccccccc}\text { RT [min] } & \text { Height } & \text { Area } & \text { Area } \% & \begin{array}{c}\text { Resolution } \\ \text { USP }\end{array} & \text { Tail } & \begin{array}{c}\text { Theoretical } \\ \text { Plates USP }\end{array} \\ 8.91 & 642.408 & 6981.429 & 97.71 & & 1.2 & 16601 \\ 16.05 & 6.959 & 163.766 & 2.29 & 15.6 & 1.1 & 10227\end{array}$


HPLC spectra of Rac-4da

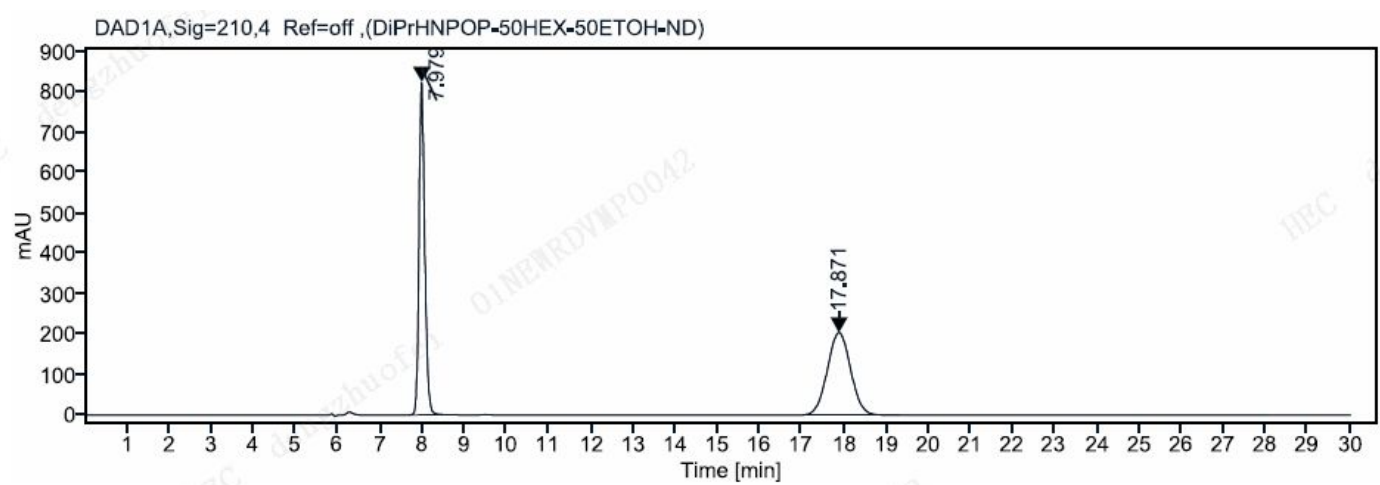

Signal: $\quad$ DAD1A,Sig=210,4 Ref=off

$\begin{array}{ccccccc}\text { RT [min] } & \text { Height } & \text { Area } & \text { Area \% } & \begin{array}{c}\text { Resolution } \\ \text { USP }\end{array} & \text { Tail } & \begin{array}{c}\text { Peak Theoretical } \\ \text { Plates USP }\end{array} \\ 7.98 & 824.056 & 7813.434 & 50.08 & & 1.2 & 16772.77903 \\ 17.87 & 203.852 & 7787.544 & 49.92 & 15.6 & 1.0 & 4901.50412\end{array}$

HPLC spectra of (R)-4da

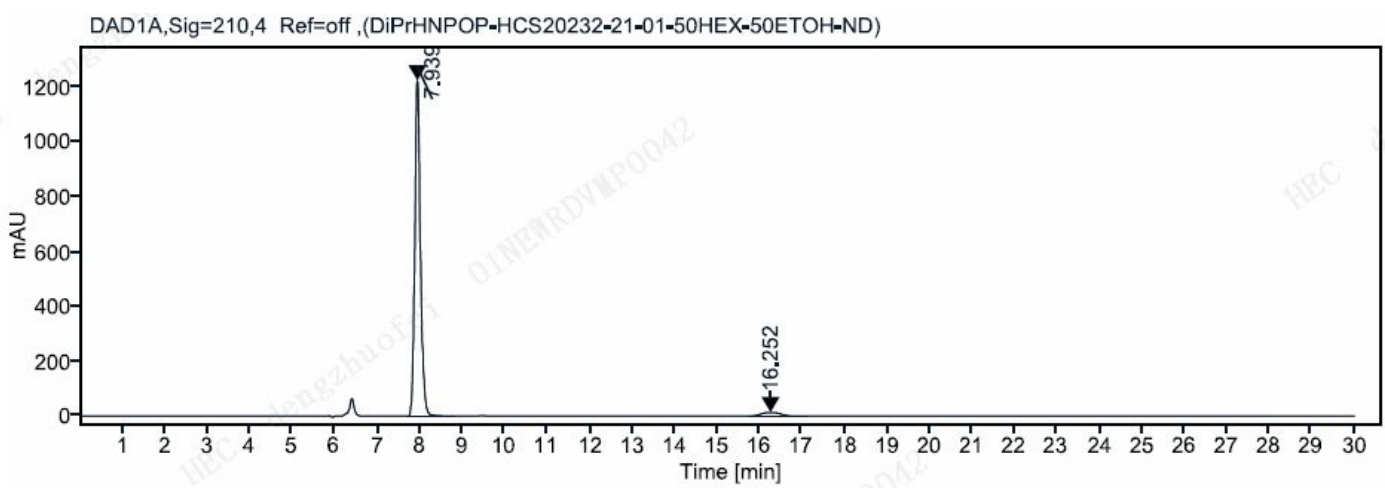

Signal: $\quad$ DAD1A,Sig=210,4 Ref=off

$\begin{array}{ccccccc}\text { RT [min] } & \text { Height } & \text { Area } & \text { Area \% } & \begin{array}{c}\text { Resolution } \\ \text { USP }\end{array} & \text { Tail } & \begin{array}{c}\text { Peak Theoretical } \\ \text { Plates USP }\end{array} \\ 7.94 & 1223.389 & 11472.169 & 96.19 & & 1.2 & 16979.34985 \\ 16.25 & 14.037 & 454.322 & 3.81 & 14.8 & 1.0 & 5494.29453\end{array}$


HPLC spectra of Rac-4ea

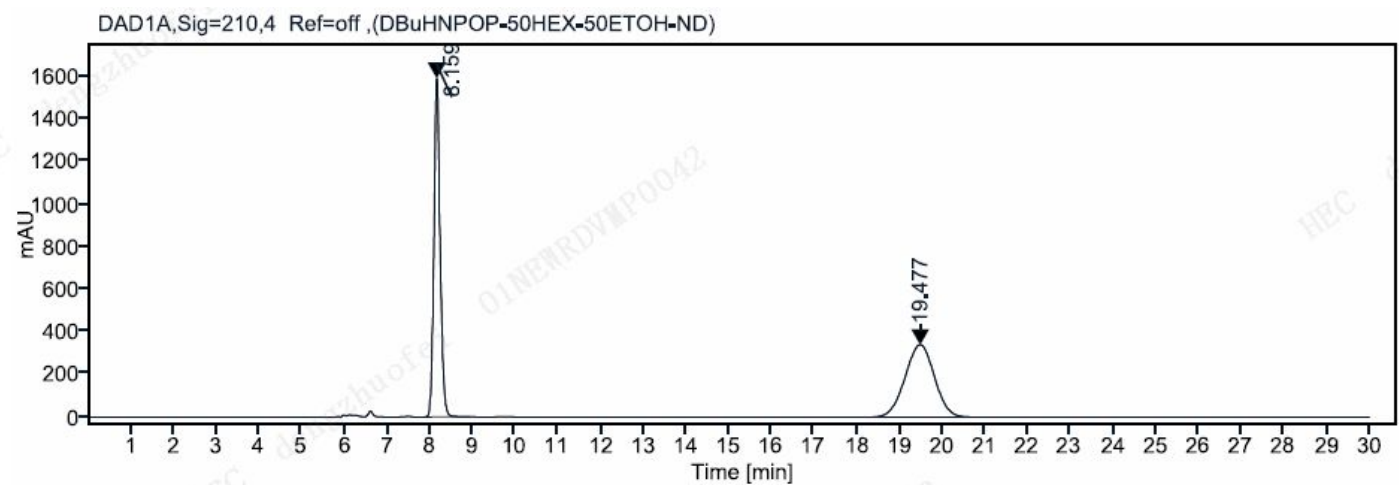

Signal: $\quad$ DAD1A,Sig=210,4 Ref=off

$\begin{array}{ccccccc}\text { RT [min] } & \text { Height } & \text { Area } & \text { Area \% } & \begin{array}{c}\text { Resolution } \\ \text { USP }\end{array} & \text { Tail } & \begin{array}{c}\text { Peak Theoretical } \\ \text { Plates USP }\end{array} \\ 8.16 & 1590.006 & 16162.225 & 49.97 & & 1.2 & 15163.69329 \\ 19.48 & 339.043 & 16182.651 & 50.03 & 14.6 & 1.0 & 3696.57175\end{array}$

HPLC spectra of (R)-4ea

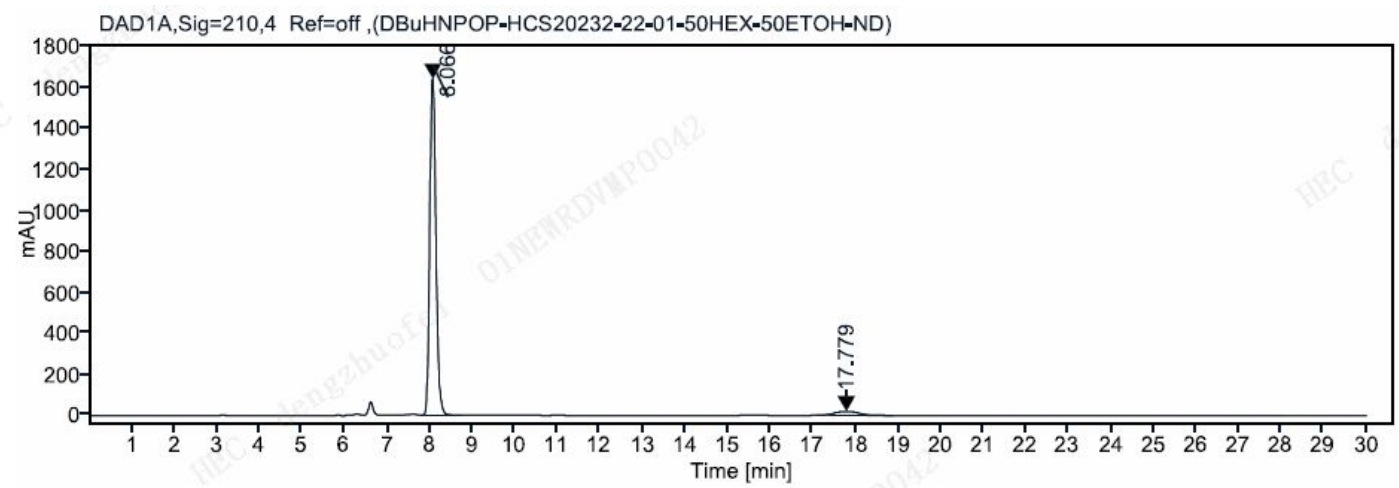

Signal: $\quad D A D 1 A, S i g=210,4$ Ref $=$ off

$\begin{array}{ccccccc}\text { RT [min] } & \text { Height } & \text { Area } & \text { Area \% } & \begin{array}{c}\text { Resolution } \\ \text { USP }\end{array} & \text { Tail } & \begin{array}{c}\text { Peak Theoretical } \\ \text { Plates USP }\end{array} \\ 8.07 & 1642.613 & 16273.083 & 95.26 & & 1.2 & 15629.66119 \\ 17.78 & 19.594 & 808.901 & 4.74 & 14.1 & 1.0 & 4043.94301\end{array}$


HPLC spectra of Rac-4fa

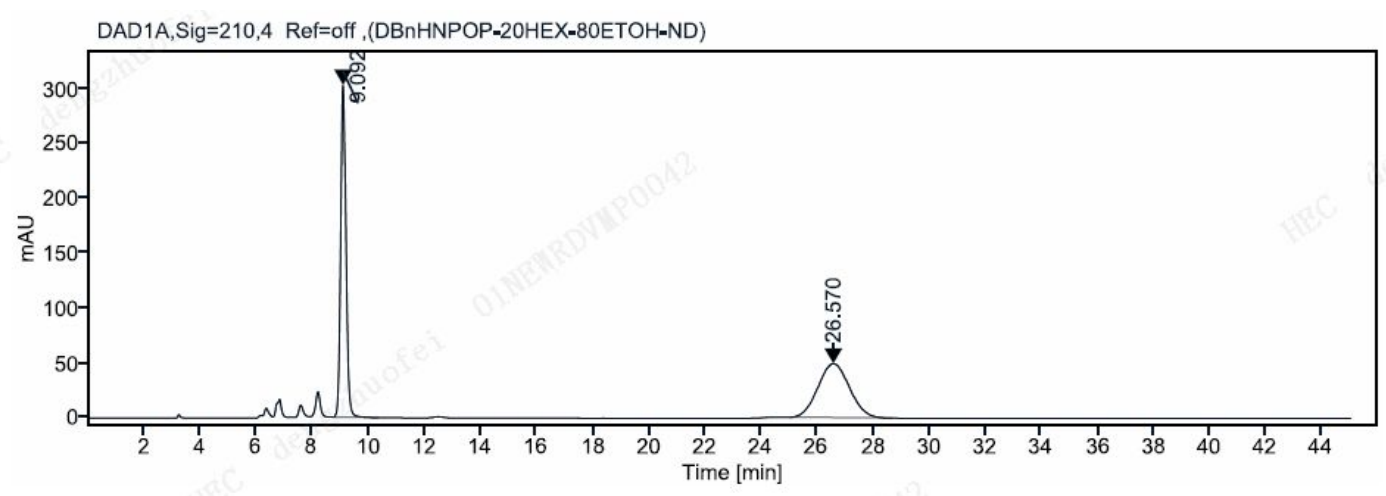

Signal: $\quad$ DAD1A,Sig $=210,4$ Ref $=$ off

$\begin{array}{ccccccc}\text { RT [min] } & \text { Height } & \text { Area } & \text { Area \% } & \begin{array}{c}\text { Resolution } \\ \text { USP }\end{array} & \text { Tail } & \begin{array}{c}\text { Peak Theoretical } \\ \text { Plates USP }\end{array} \\ 9.09 & 302.644 & 3988.289 & 50.96 & & 1.1 & 11465.39371 \\ 26.57 & 49.125 & 3837.787 & 49.04 & 14.3 & 1.0 & 2568.67703\end{array}$

HPLC spectra of (R)-4fa

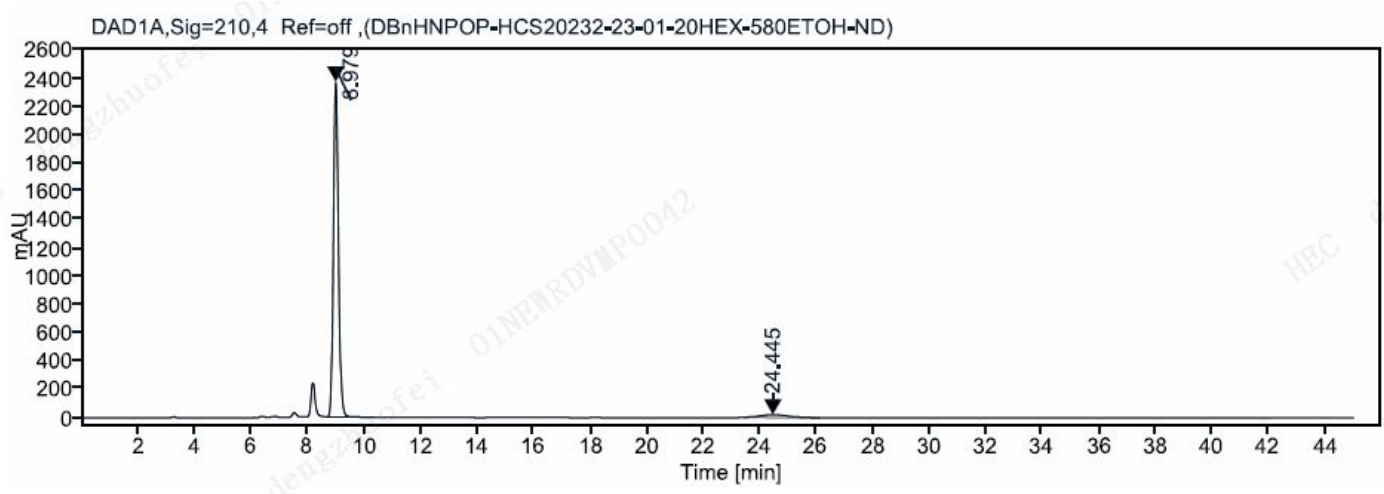

Signal: $\quad$ DAD1A,Sig=210,4 Ref=off

$\begin{array}{ccccccc}\text { RT [min] } & \text { Height } & \text { Area } & \text { Area \% } & \begin{array}{c}\text { Resolution } \\ \text { USP }\end{array} & \text { Tail } & \begin{array}{c}\text { Peak Theoretical } \\ \text { Plates USP }\end{array} \\ 8.98 & 2376.089 & 29827.315 & 95.63 & & 1.2 & 12191.16906 \\ 24.44 & 20.657 & 1362.307 & 4.37 & 14.6 & 1.0 & 2968.26676\end{array}$


HPLC spectra of Rac-4cb

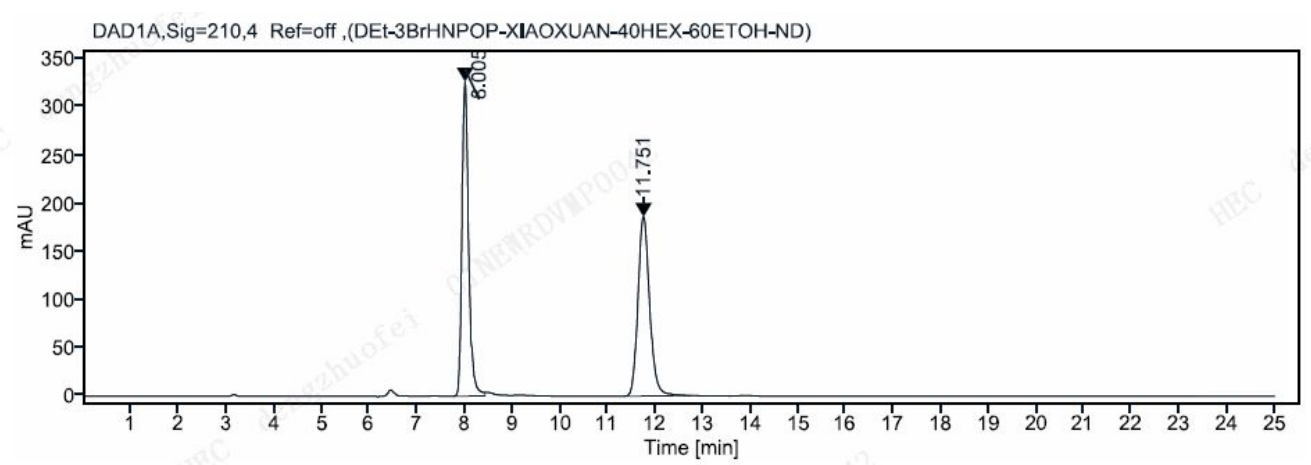

Signal: DAD1A,Sig=210,4 Ref=off

$\begin{array}{ccccccc}\text { RT [min] } & \text { Height } & \text { Area } & \text { Area \% } & \begin{array}{c}\text { Resolution } \\ \text { USP }\end{array} & \text { Tail } & \begin{array}{c}\text { Theoretical } \\ \text { Plates USP }\end{array} \\ 8.01 & 325.788 & 3136.518 & 49.85 & & 1.3 & 17110 \\ 11.75 & 185.340 & 3154.875 & 50.15 & 10.9 & 1.2 & 11399\end{array}$

HPLC spectra of (R)-4cb

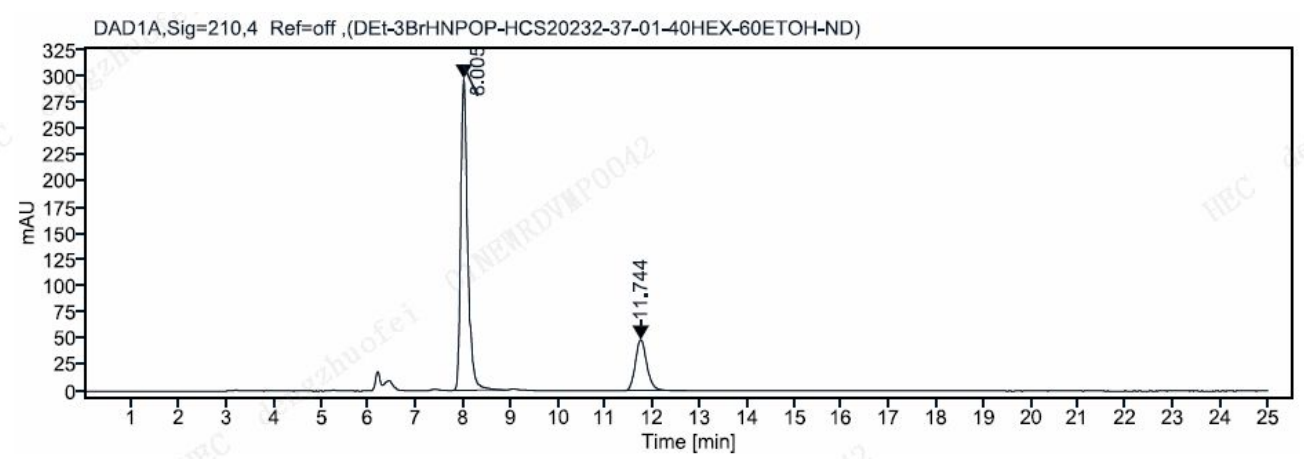

Signal: $\quad$ DAD1A,Sig $=210,4$ Ref=off

$\begin{array}{ccccccc}\text { RT [min] } & \text { Height } & \text { Area } & \text { Area \% } & \begin{array}{c}\text { Resolution } \\ \text { USP }\end{array} & \begin{array}{c}\text { Tail } \\ \text { Theoretical }\end{array} & \begin{array}{c}\text { Plates USP } \\ 8.01\end{array} \\ 297.448 & 3014.803 & 78.49 & & 1.3 & 16194 \\ 11.74 & 48.605 & 826.217 & 21.51 & 10.8 & 1.2 & 11384\end{array}$


HPLC spectra of Rac-4cc

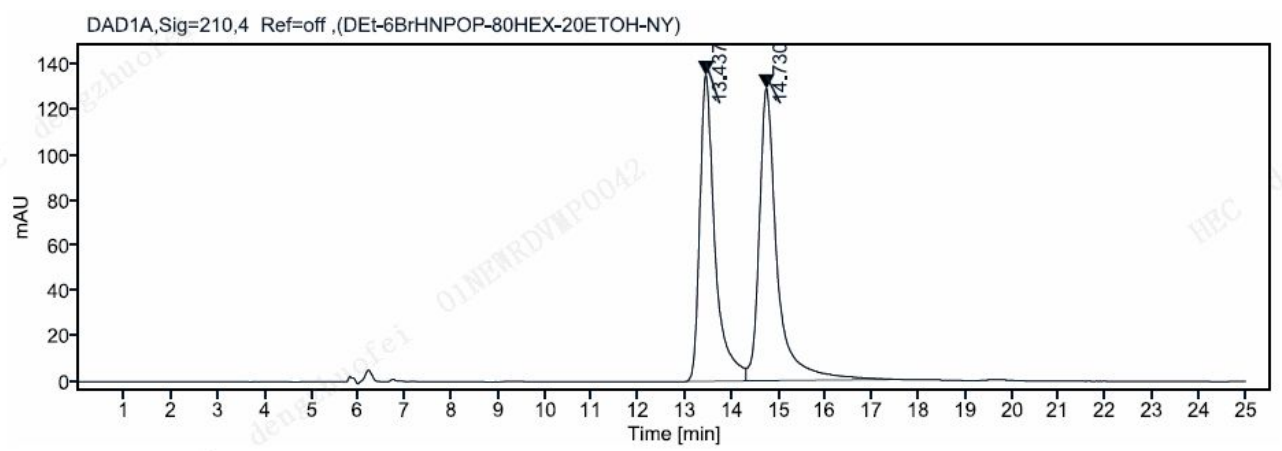

Signal: $\quad$ DAD1A,Sig $=210,4$ Ref $=$ off

$\begin{array}{ccccccc}\text { RT [min] } & \text { Height } & \text { Area } & \text { Area \% } & \begin{array}{c}\text { Resolution } \\ \text { USP }\end{array} & \text { Tail } & \begin{array}{c}\text { Theoretical } \\ \text { Plates USP }\end{array} \\ 13.44 & 135.307 & 3056.405 & 47.07 & & 1.8 & 10037 \\ 14.73 & 129.066 & 3436.942 & 52.93 & 2.3 & 1.6 & 9526\end{array}$

HPLC spectra of (R)-4cc

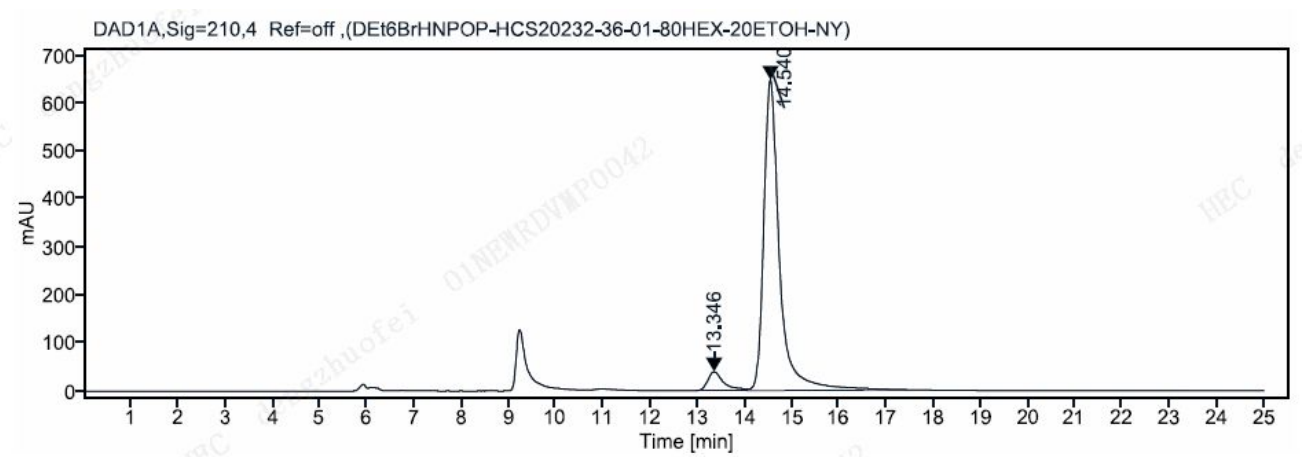

Signal: DAD1A,Sig=210,4 Ref=off

$\begin{array}{ccccccc}\text { RT [min] } & \text { Height } & \text { Area } & \text { Area \% } & \begin{array}{c}\text { Resolution } \\ \text { USP }\end{array} & \begin{array}{c}\text { Tail } \\ \text { Theoretical }\end{array} & \begin{array}{c}\text { Plates USP } \\ 13.35\end{array} \\ 39.355 & 920.330 & 5.79 & & 1.8 & 9200 \\ 14.54 & 647.642 & 14971.906 & 94.21 & 2.2 & 1.5 & 11453\end{array}$


HPLC spectra of Rac-4cd

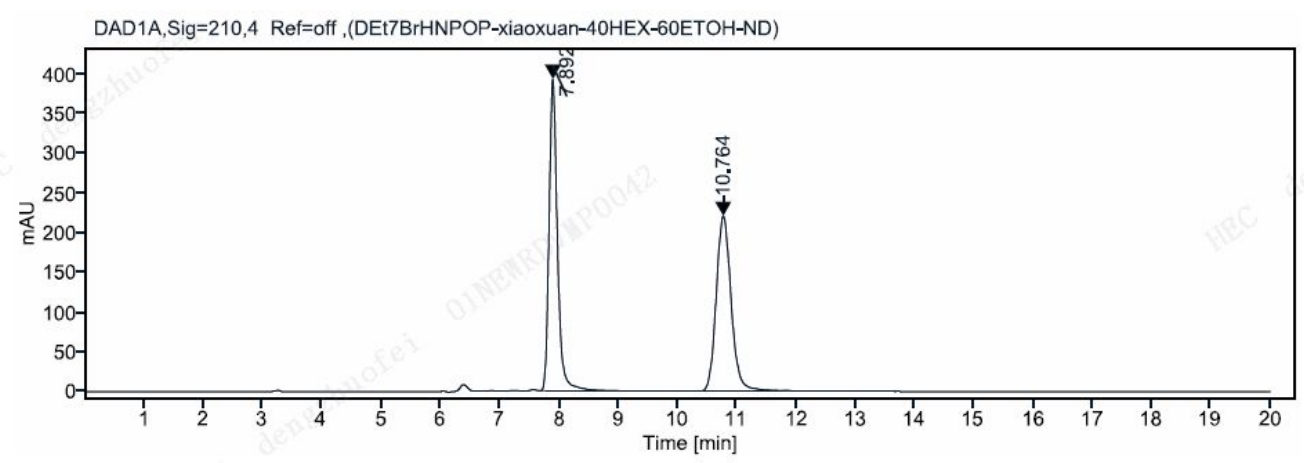

Signal: $\quad D A D 1 A, S i g=210,4$ Ref $=$ off

\begin{tabular}{|c|c|c|c|c|c|c|}
\hline RT [min] & Height & Area & Area \% & $\begin{array}{l}\text { Resolution } \\
\text { USP }\end{array}$ & Tail & $\begin{array}{l}\text { Theoretical } \\
\text { Plates USP }\end{array}$ \\
\hline 7.89 & 392.785 & 3841.615 & 49.93 & & 1.3 & 16677 \\
\hline 10.76 & 220.823 & 3852.178 & 50.07 & 8.3 & 1.1 & 9170 \\
\hline
\end{tabular}

HPLC spectra of (R)-4cd

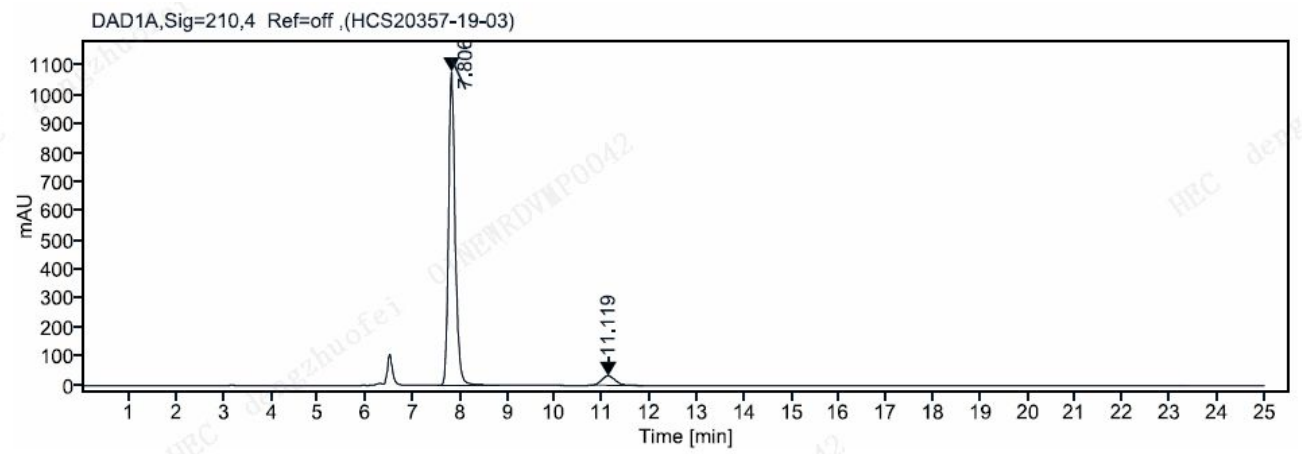

Signal: $\quad$ DAD1A, Sig $=210,4$ Ref $=$ off

$\begin{array}{ccccccc}\text { RT [min] } & \text { Height } & \text { Area } & \text { Area \% } & \begin{array}{c}\text { Resolution } \\ \text { USP }\end{array} & \text { Tail } & \begin{array}{c}\text { Theoretical } \\ \text { Plates USP }\end{array} \\ 7.81 & 1079.974 & 10704.295 & 94.07 & & 1.2 & 15132 \\ 11.12 & 32.812 & 675.104 & 5.93 & 8.3 & 1.1 & 6636\end{array}$


HPLC spectra of Rac-4ce

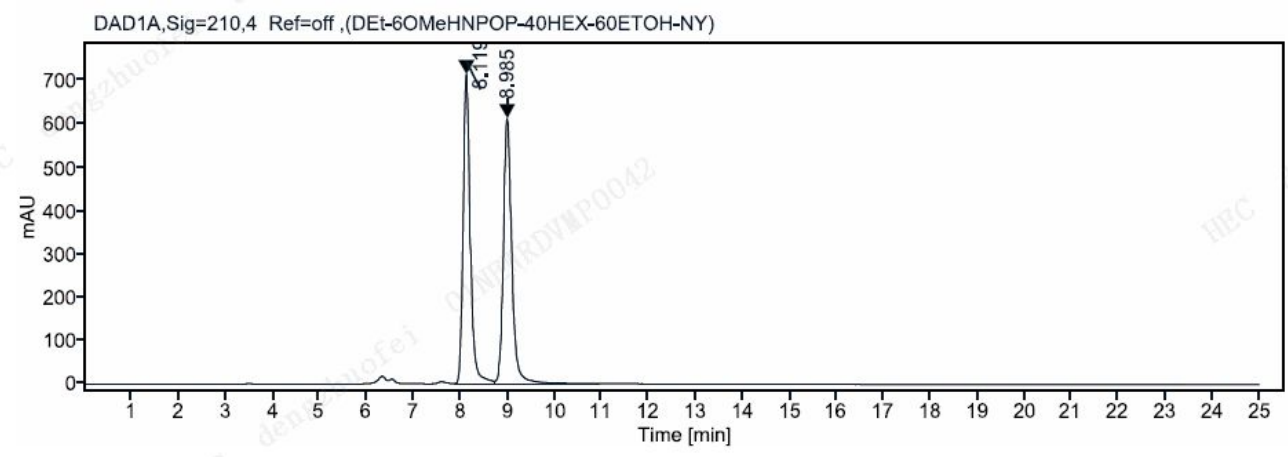

Signal: $\quad$ DAD1A,Sig $=210,4$ Ref $=$ off

\begin{tabular}{|c|c|c|c|c|c|c|}
\hline RT [min] & Height & Area & Area $\%$ & $\begin{array}{c}\text { Resolution } \\
\text { USP }\end{array}$ & Tail & $\begin{array}{l}\text { Theoretical } \\
\text { Plates USP }\end{array}$ \\
\hline 8.12 & 713.844 & 7595.677 & 49.62 & & 1.3 & 14959 \\
\hline 8.99 & 611.947 & 7713.313 & 50.38 & 3.0 & 1.2 & 13282 \\
\hline
\end{tabular}

HPLC spectra of (R)-4ce

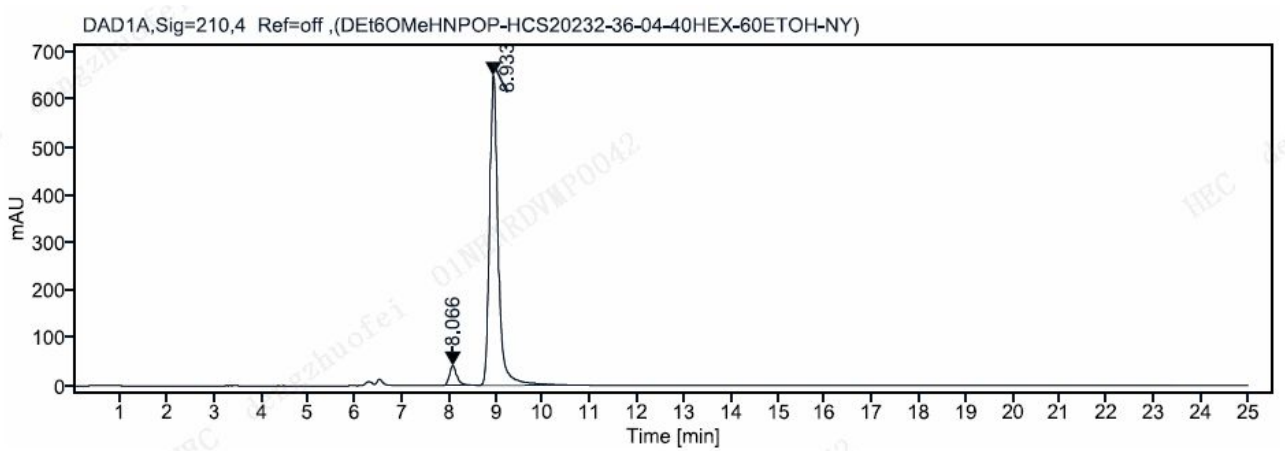

Signal: DAD1A,Sig=210,4 Ref=off

$\begin{array}{ccccccc}\text { RT [min] } & \text { Height } & \text { Area } & \text { Area \% } & \begin{array}{c}\text { Resolution } \\ \text { USP }\end{array} & \text { Tail } & \begin{array}{c}\text { Theoretical } \\ \text { Plates USP }\end{array} \\ 8.07 & 42.037 & 483.942 & 5.46 & & 1.4 & 13259 \\ 8.93 & 651.114 & 8385.746 & 94.54 & 2.9 & 1.3 & 12974\end{array}$


HPLC spectra of Rac-4cf

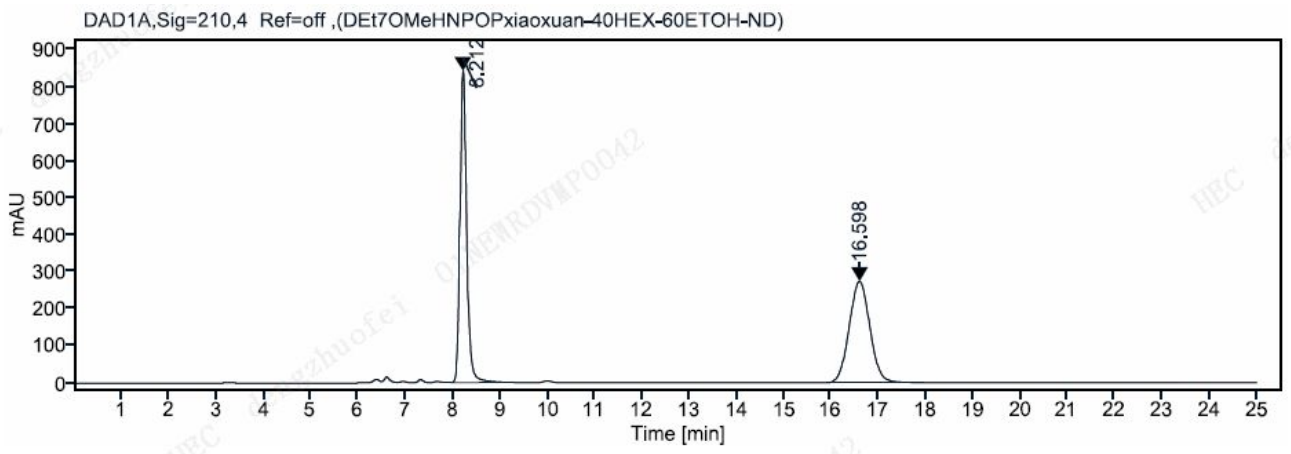

Signal: DAD1A,Sig=210,4 Ref=off

\begin{tabular}{ccccccc} 
RT [min] & Height & Area & Area \% & $\begin{array}{c}\text { Resolution } \\
\text { USP }\end{array}$ & $\begin{array}{c}\text { Tail } \\
\text { Theoretical }\end{array}$ & $\begin{array}{c}\text { Plates USP } \\
\text { Pla }\end{array}$ \\
\hline 8.21 & 842.506 & 8330.605 & 49.92 & & 1.2 & 17187 \\
16.60 & 273.970 & 8358.202 & 50.08 & 15.8 & 1.1 & 6737
\end{tabular}

HPLC spectra of (R)-4cf

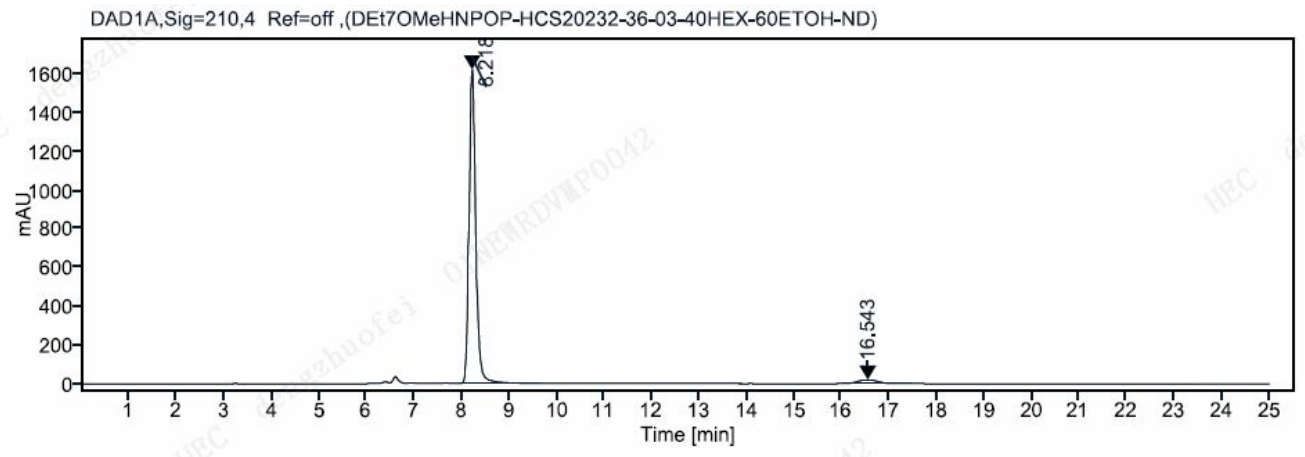

Signal: $\quad$ DAD1A,Sig=210,4 Ref=off

$\begin{array}{ccccccc}\text { RT [min] } & \text { Height } & \text { Area } & \text { Area \% } & \begin{array}{c}\text { Resolution } \\ \text { USP }\end{array} & \text { Tail } & \begin{array}{c}\text { Theoretical } \\ \text { Plates USP }\end{array} \\ 8.22 & 1625.067 & 16170.873 & 96.56 & & 1.2 & 17090 \\ 16.54 & 19.489 & 576.051 & 3.44 & 15.9 & 1.1 & 6978\end{array}$


HPLC spectra of Rac-4cg

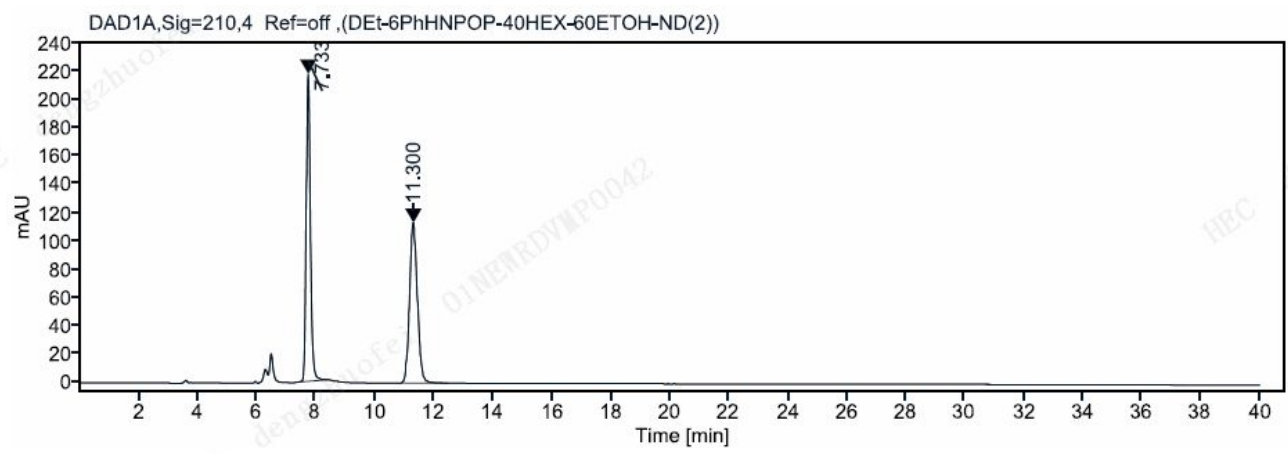

Signal: $\quad$ DAD1A,Sig $=210,4$ Ref $=$ off

$\begin{array}{ccccccc}\text { RT [min] } & \text { Height } & \text { Area } & \text { Area \% } & \begin{array}{c}\text { Resolution } \\ \text { USP }\end{array} & \text { Tail } & \begin{array}{c}\text { Theoretical } \\ \text { Plates USP }\end{array} \\ 7.73 & 217.311 & 2122.700 & 50.27 & & 1.2 & 15334 \\ 11.30 & 113.304 & 2100.260 & 49.73 & 9.7 & 1.1 & 8566\end{array}$

HPLC spectra of (R)-4cg

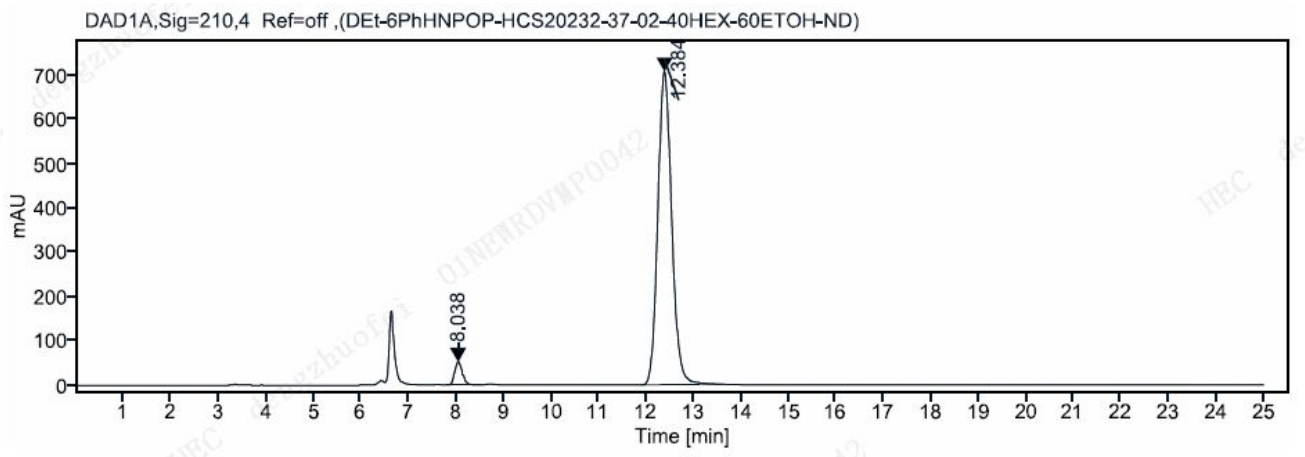

Signal: $\quad$ DAD1A,Sig $=210,4$ Ref=off

$\begin{array}{ccccccc}\text { RT [min] } & \text { Height } & \text { Area } & \text { Area \% } & \begin{array}{c}\text { Resolution } \\ \text { USP }\end{array} & \text { Tail } & \begin{array}{c}\text { Theoretical } \\ \text { Plates USP }\end{array} \\ 8.04 & 51.695 & 591.303 & 3.94 & & 1.2 & 11826 \\ 12.38 & 708.223 & 14401.616 & 96.06 & 10.5 & 1.1 & 8712\end{array}$


HPLC spectra of Rac-4ch

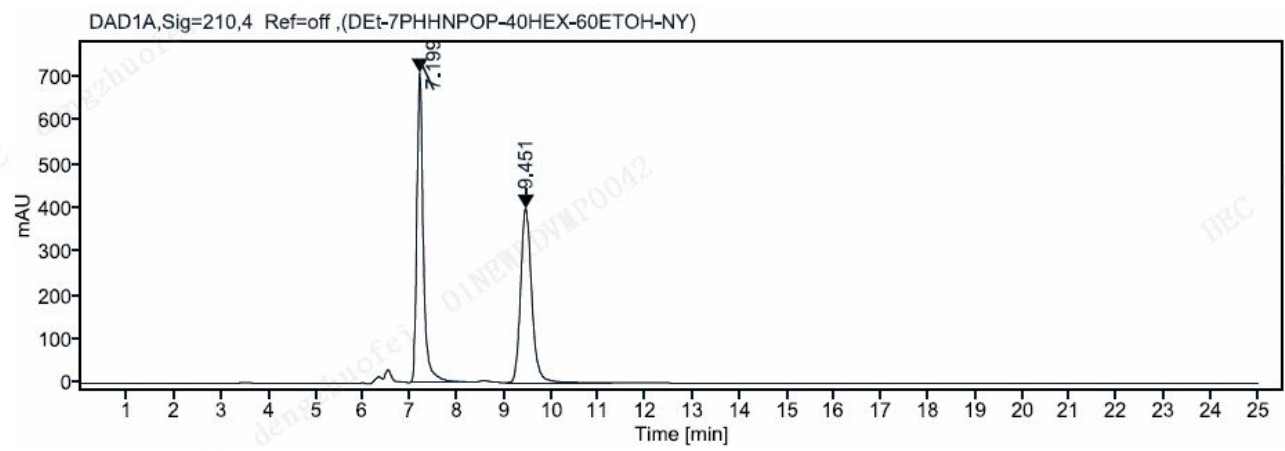

Signal: $\quad D A D 1 A, S i g=210,4$ Ref $=$ off

$\begin{array}{ccccccc}\text { RT [min] } & \text { Height } & \text { Area } & \text { Area \% } & \begin{array}{c}\text { Resolution } \\ \text { USP }\end{array} & \text { Tail } & \begin{array}{c}\text { Theoretical } \\ \text { Plates USP }\end{array} \\ 7.20 & 705.094 & 6682.027 & 50.54 & & 1.4 & 15259 \\ 9.45 & 397.344 & 6539.421 & 49.46 & 6.9 & 1.2 & 8082\end{array}$

HPLC spectra of (R)-4ch

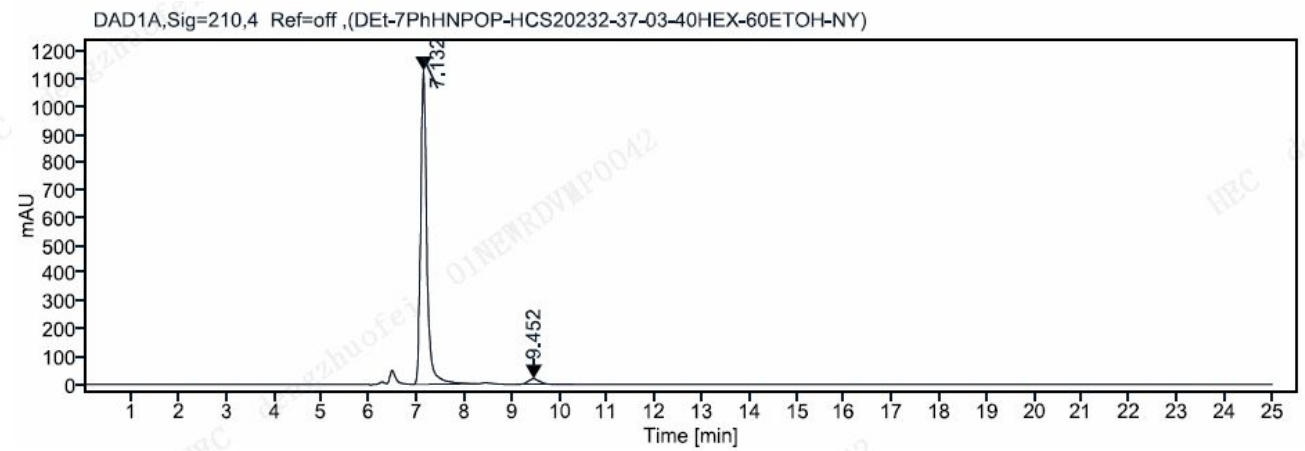

Signal: $\quad$ DAD1A,Sig $=210,4$ Ref $=$ off

$\begin{array}{ccccccc}\text { RT [min] } & \text { Height } & \text { Area } & \text { Area } \% & \begin{array}{c}\text { Resolution } \\ \text { USP }\end{array} & \text { Tail } & \begin{array}{c}\text { Theoretical } \\ \text { Plates USP }\end{array} \\ 7.13 & 1131.027 & 10364.231 & 96.77 & & 1.3 & 16306 \\ 9.45 & 19.754 & 346.250 & 3.23 & 6.9 & 1.2 & 7018\end{array}$


HPLC spectra of Rac-4ci

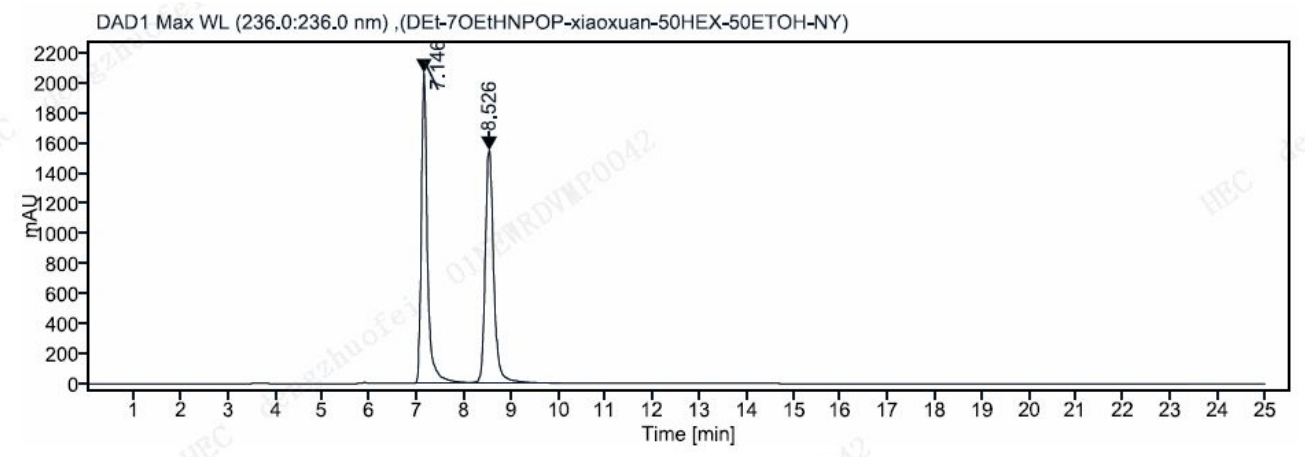

Signal: $\quad$ DAD1 Max WL $(236.0: 236.0 \mathrm{~nm})$

\begin{tabular}{|c|c|c|c|c|c|c|}
\hline $\mathrm{RT}$ [min] & Height & Area & Area $\%$ & $\begin{array}{l}\text { Resolution } \\
\text { USP }\end{array}$ & Tail & $\begin{array}{l}\text { Theoretica } \\
\text { Plates USP }\end{array}$ \\
\hline 7.15 & 2069.367 & 19146.557 & 49.56 & & 1.5 & 17057 \\
\hline 8.53 & 1551.180 & 19488.259 & 50.44 & 5.2 & 1.2 & 12041 \\
\hline
\end{tabular}

HPLC spectra of (R)-4ci

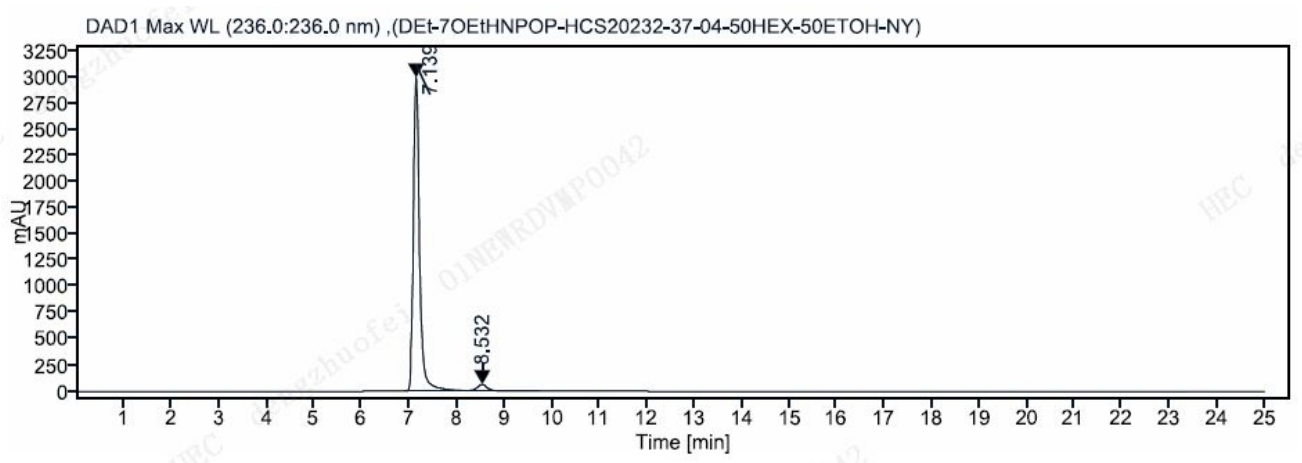

Signal: $\quad$ DAD1 Max WL (236.0:236.0 nm)

\begin{tabular}{|c|c|c|c|c|c|c|}
\hline RT [min] & Height & Area & Area \% & $\begin{array}{c}\text { Resolution } \\
\text { USP }\end{array}$ & Tail & $\begin{array}{l}\text { Theoretical } \\
\text { Plates USP }\end{array}$ \\
\hline 7.14 & 2997.449 & 28006.554 & 96.10 & & 1.3 & 15999 \\
\hline 8.53 & 65.278 & 1136.152 & 3.90 & 4.5 & 1.1 & 7589 \\
\hline
\end{tabular}


HPLC spectra of Rac-4cj

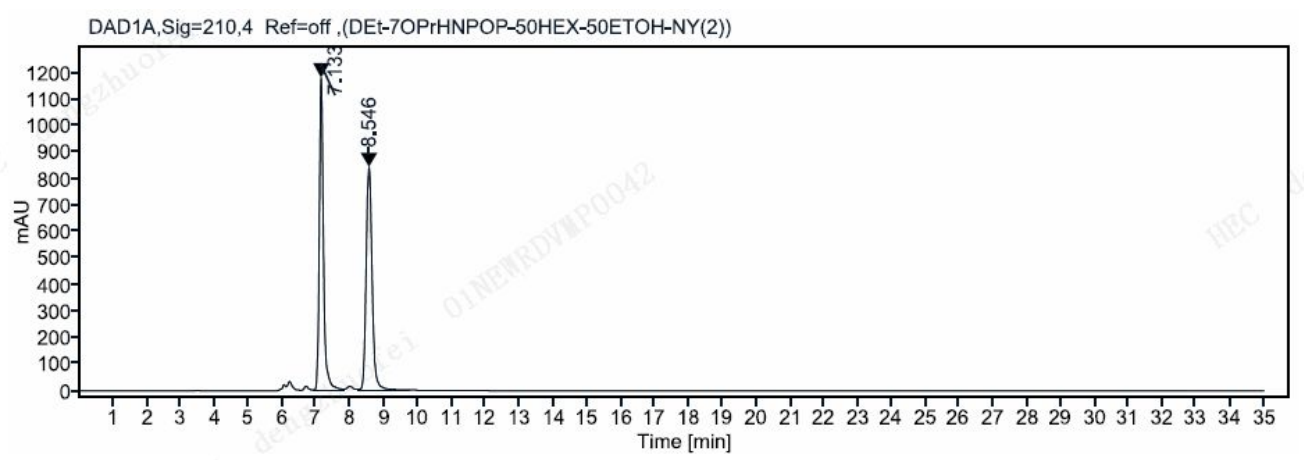

Signal: $\quad$ DAD1A,Sig=210,4 Ref=off

$\begin{array}{ccccccc}\text { RT [min] } & \text { Height } & \text { Area } & \text { Area \% } & \begin{array}{c}\text { Resolution } \\ \text { USP }\end{array} & \text { Tail } & \begin{array}{c}\text { Theoretical } \\ \text { Plates USP }\end{array} \\ 7.13 & 1180.318 & 10281.310 & 50.14 & & 1.4 & 17896 \\ 8.55 & 843.488 & 10222.020 & 49.86 & 5.4 & 1.2 & 12369\end{array}$

HPLC spectra of (R)-4cj

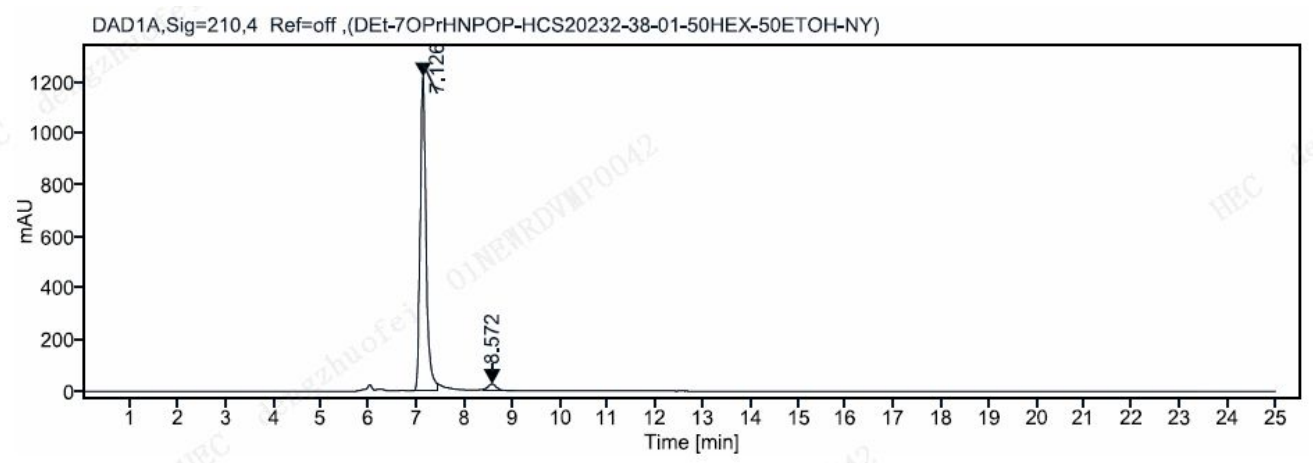

Signal: $\quad$ DAD1A,Sig $=210,4$ Ref $=$ off

$\begin{array}{ccccccc}\text { RT [min] } & \text { Height } & \text { Area } & \text { Area \% } & \begin{array}{c}\text { Resolution } \\ \text { USP }\end{array} & \text { Tail } & \begin{array}{c}\text { Theoretical } \\ \text { Plates USP }\end{array} \\ 7.13 & 1219.661 & 10588.276 & 96.94 & & 1.3 & 17364 \\ 8.57 & 26.237 & 334.680 & 3.06 & 5.3 & 1.2 & 10836\end{array}$


HPLC spectra of Rac-4ck

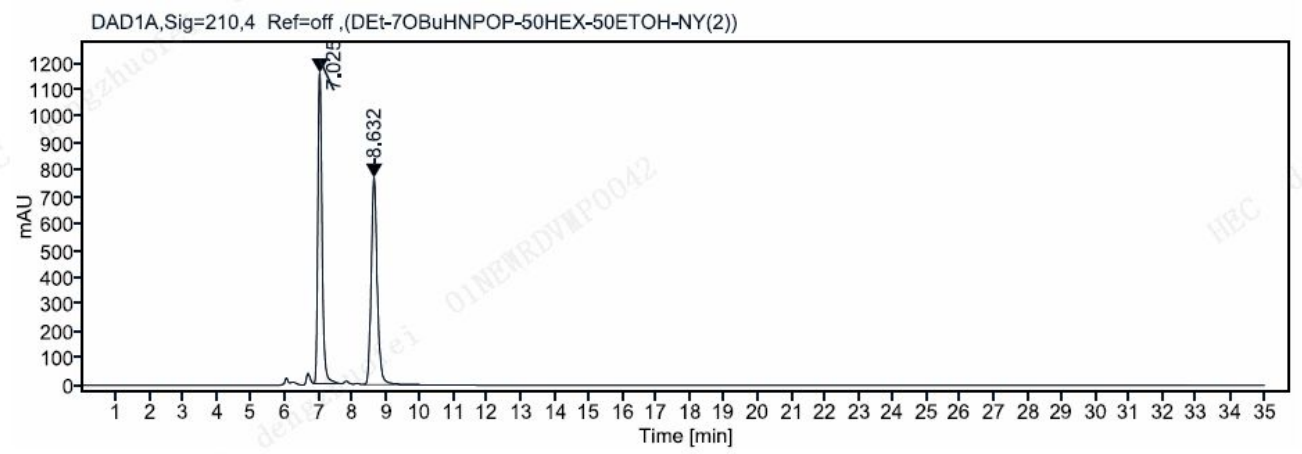

Signal: $\quad$ DAD1A,Sig $=210,4$ Ref $=$ off

$\begin{array}{ccccccc}\text { RT [min] } & \text { Height } & \text { Area } & \text { Area \% } & \begin{array}{c}\text { Resolution } \\ \text { USP }\end{array} & \text { Tail } & \begin{array}{c}\text { Theoretical } \\ \text { Plates USP }\end{array} \\ 7.03 & 1159.305 & 9747.117 & 49.85 & & 1.3 & 18070 \\ 8.63 & 771.230 & 9806.363 & 50.15 & 6.0 & 1.2 & 11386\end{array}$

HPLC spectra of (R)-4ck

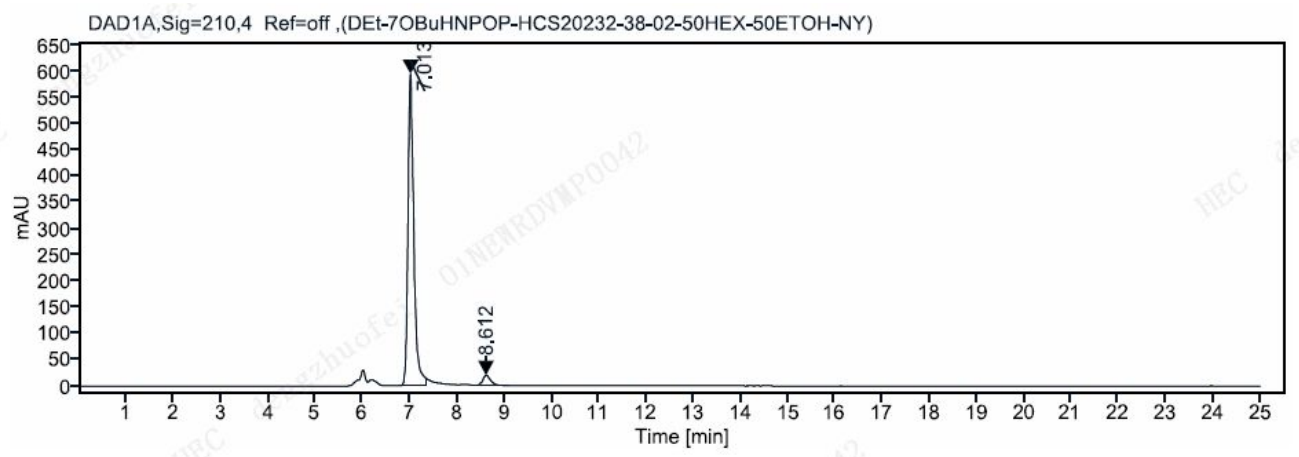

Signal: $\quad$ DAD1A,Sig $=210,4$ Ref $=$ off

\begin{tabular}{ccccccc} 
RT [min] & Height & Area & Area \% & $\begin{array}{c}\text { Resolution } \\
\text { USP }\end{array}$ & $\begin{array}{c}\text { Tail } \\
\text { Theoretical }\end{array}$ & $\begin{array}{c}\text { Plates USP } \\
7.01\end{array}$ \\
\hline 594.576 & 5128.108 & 95.64 & & 1.4 & 17139 \\
8.61 & 20.073 & 233.701 & 4.36 & 6.3 & 1.3 & 13920
\end{tabular}


HPLC spectra of Rac-4l

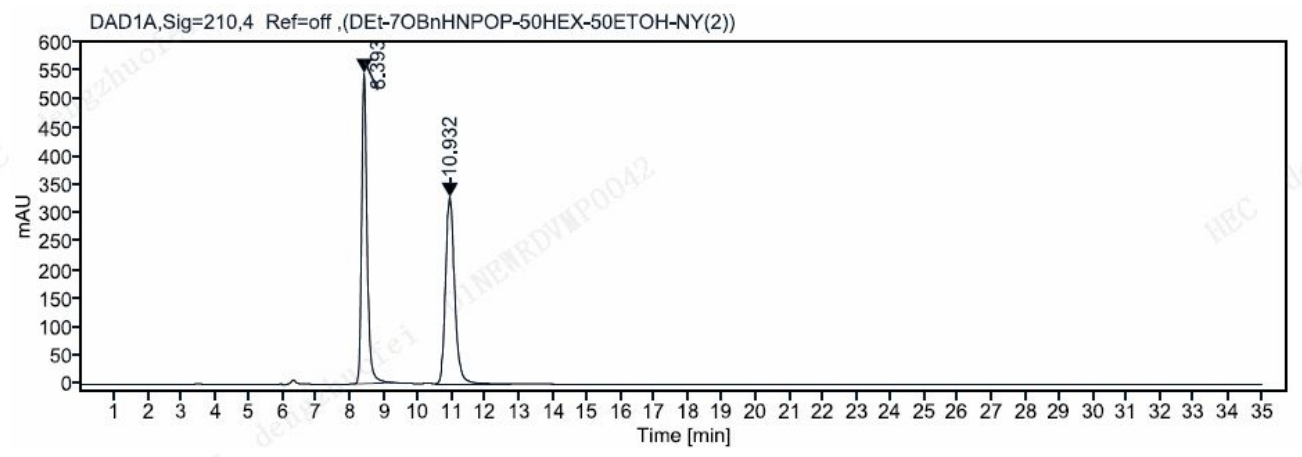

Signal: $\quad$ DAD1A,Sig $=210,4$ Ref $=$ off

$\begin{array}{ccccccc}\text { RT [min] } & \text { Height } & \text { Area } & \text { Area \% } & \begin{array}{c}\text { Resolution } \\ \text { USP }\end{array} & \text { Tail } & \begin{array}{c}\text { Theoretical } \\ \text { Plates USP }\end{array} \\ 8.39 & 545.260 & 6164.996 & 49.89 & & 1.3 & 13930 \\ 10.93 & 328.232 & 6191.510 & 50.11 & 6.6 & 1.2 & 8150\end{array}$

HPLC spectra of (R)-4l

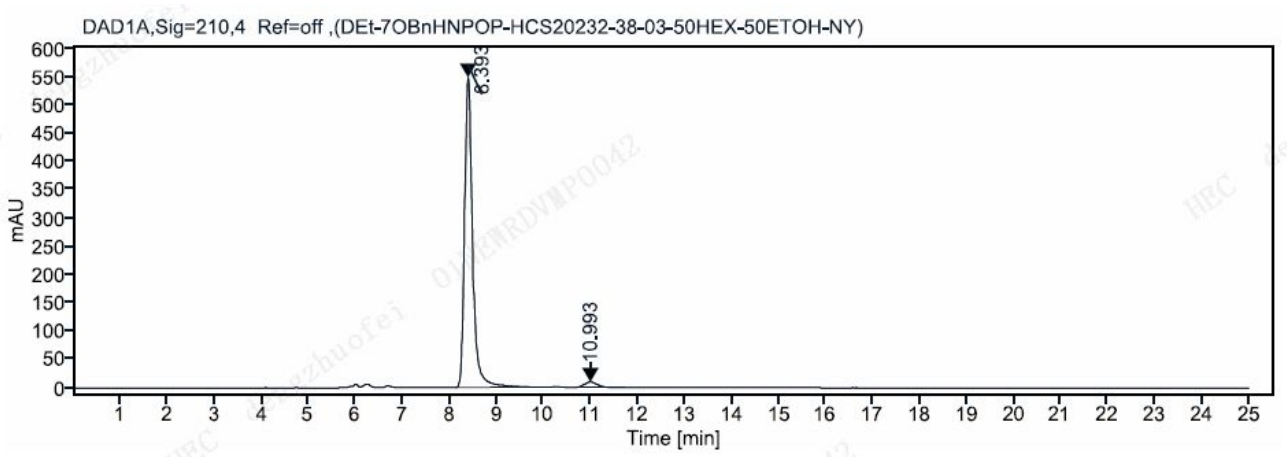

Signal: $\quad$ DAD1A,Sig $=210,4$ Ref $=$ off

$\begin{array}{ccccccc}\text { RT [min] } & \text { Height } & \text { Area } & \text { Area \% } & \begin{array}{c}\text { Resolution } \\ \text { USP }\end{array} & \text { Tail } & \begin{array}{c}\text { Theoretical } \\ \text { Plates USP }\end{array} \\ 8.39 & 548.910 & 6496.768 & 97.30 & & 1.3 & 13371 \\ 10.99 & 9.424 & 180.022 & 2.70 & 6.6 & 1.2 & 7627\end{array}$


HPLC spectra of Rac-4cm

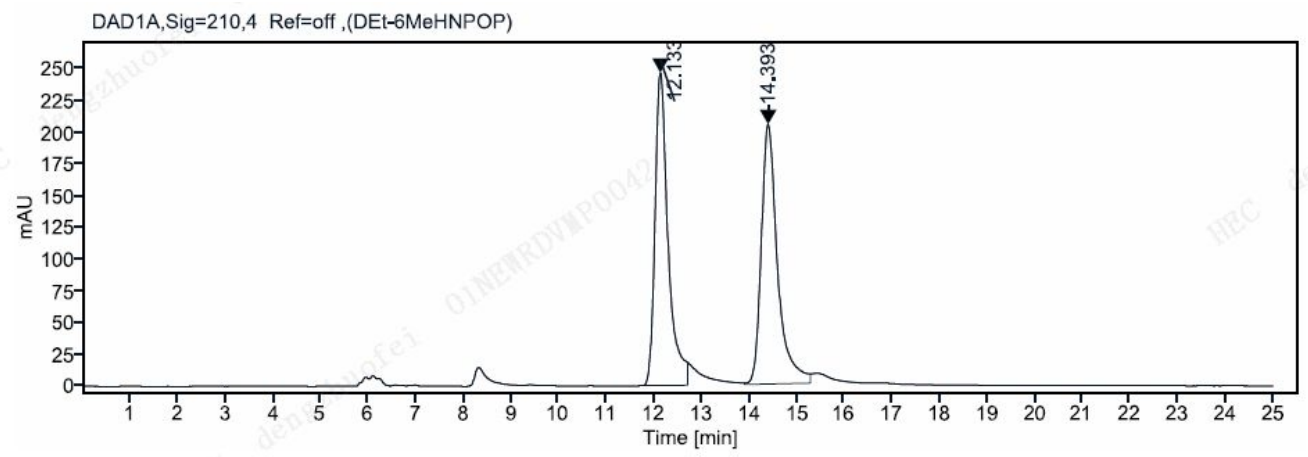

Signal: $\quad D A D 1 A, S i g=210,4$ Ref $=$ off

$\begin{array}{ccccccc}\text { RT [min] } & \text { Height } & \text { Area } & \text { Area \% } & \begin{array}{c}\text { Resolution } \\ \text { USP }\end{array} & \begin{array}{c}\text { Tail } \\ \text { Theoretical }\end{array} & \begin{array}{c}\text { Plates USP } \\ 12.13\end{array} \\ 246.228 & 4686.744 & 49.11 & & 1.7 & 10894 \\ 14.39 & 204.523 & 4856.204 & 50.89 & 4.4 & 1.6 & 10077\end{array}$

HPLC spectra of (R)-4cm

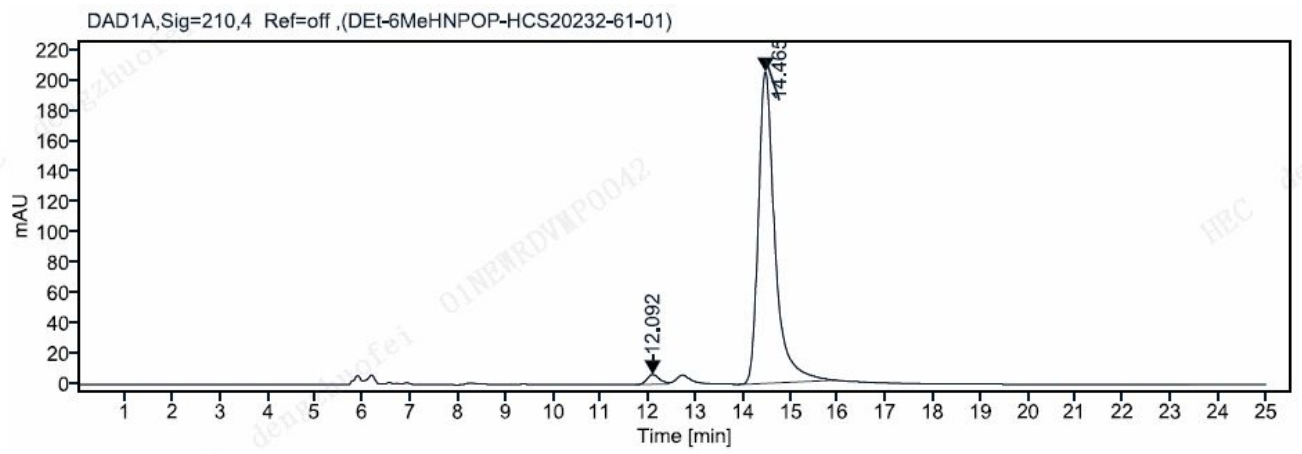

Signal: $\quad$ DAD1A,Sig $=210,4$ Ref $=$ off

\begin{tabular}{ccccccc} 
RT [min] & Height & Area & Area \% & $\begin{array}{c}\text { Resolution } \\
\text { USP }\end{array}$ & $\begin{array}{c}\text { Tail } \\
\text { Theoretical }\end{array}$ & $\begin{array}{c}\text { Plates USP } \\
12.09\end{array}$ \\
\hline 6.447 & 119.082 & 2.34 & & 1.2 & 9661 \\
14.47 & 204.408 & 4965.037 & 97.66 & 4.4 & 1.5 & 9534
\end{tabular}


HPLC spectra of Rac-4cn

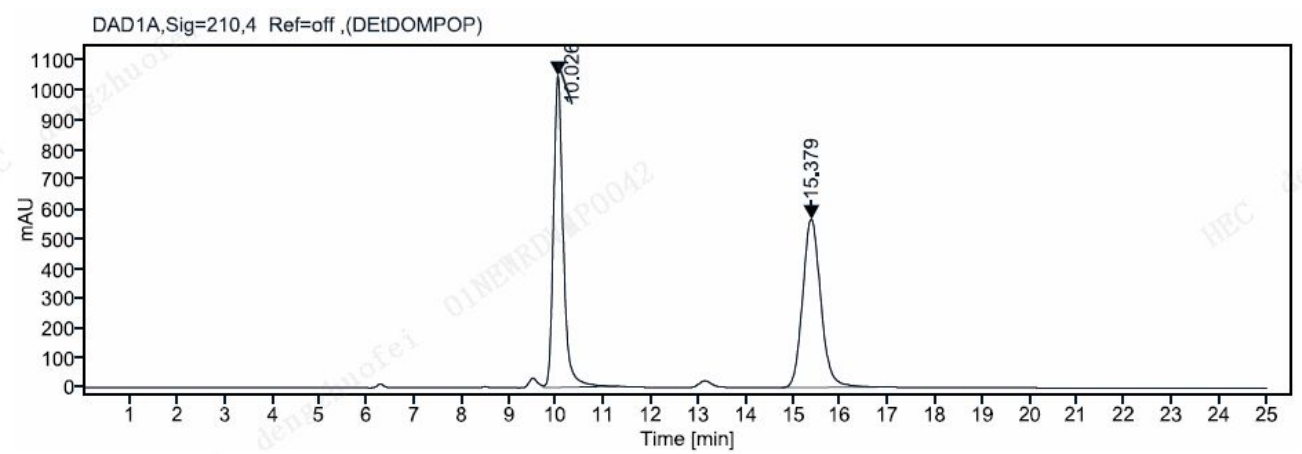

Signal: $\quad D A D 1 A, S i g=210,4$ Ref $=$ off

$\begin{array}{ccccccc}\text { RT [min] } & \text { Height } & \text { Area } & \text { Area \% } & \begin{array}{c}\text { Resolution } \\ \text { USP }\end{array} & \begin{array}{c}\text { Tail } \\ \text { Theoretical }\end{array} & \begin{array}{c}\text { Thates USP } \\ \text { Plat }\end{array} \\ 10.03 & 1049.306 & 14986.831 & 49.74 & & 1.3 & 12605 \\ 15.38 & 567.222 & 15143.990 & 50.26 & 10.2 & 1.1 & 7866\end{array}$

HPLC spectra of (R)-4cn

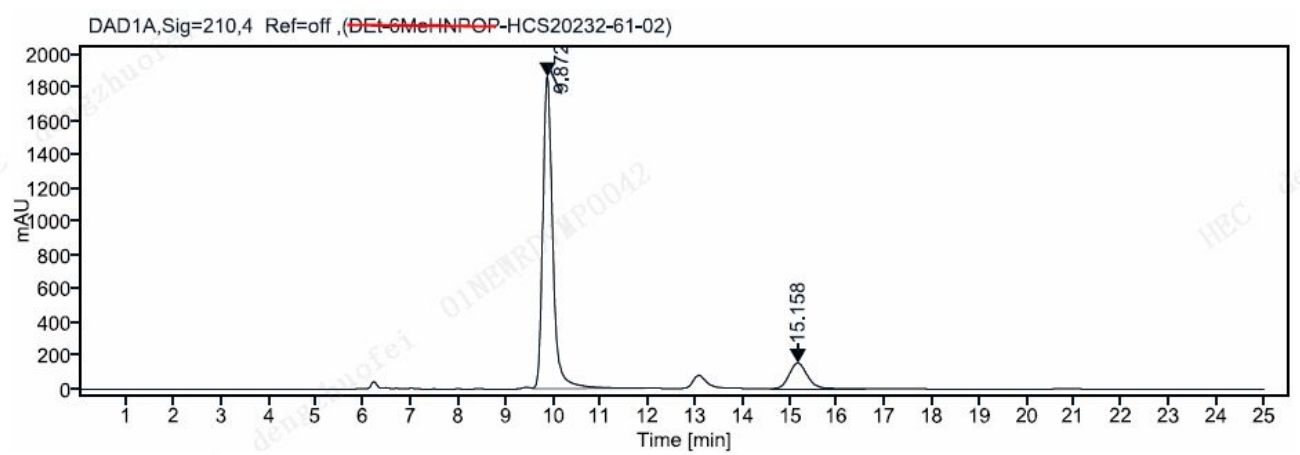

Signal: $\quad$ DAD1A,Sig $=210,4$ Ref $=$ off

\begin{tabular}{|c|c|c|c|c|c|c|}
\hline RT [min] & Height & Area & Area \% & $\begin{array}{l}\text { Resolution } \\
\text { USP }\end{array}$ & Tail & $\begin{array}{l}\text { Theoretical } \\
\text { Plates USP }\end{array}$ \\
\hline 9.87 & 1864.573 & 27501.756 & 86.99 & & 1.3 & 12021 \\
\hline 15.16 & 153.935 & 4114.260 & 13.01 & 10.0 & 1.1 & 7597 \\
\hline
\end{tabular}


HPLC spectra of Rac-5

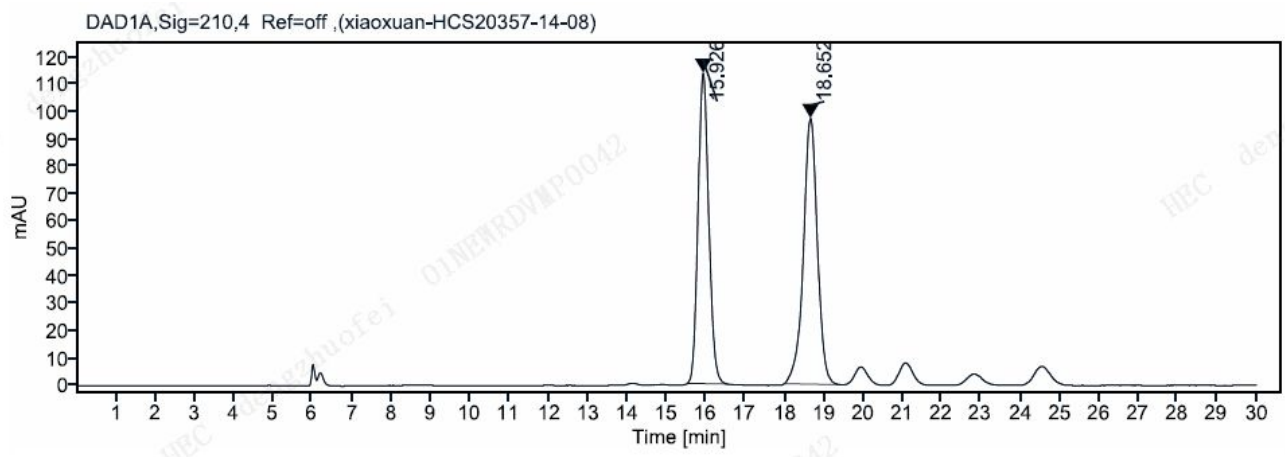

Signal: $\quad$ DAD1A,Sig $=210,4$ Ref $=$ off

$\begin{array}{ccccccc}\text { RT [min] } & \text { Height } & \text { Area } & \text { Area \% } & \begin{array}{c}\text { Resolution } \\ \text { USP }\end{array} & \text { Tail } & \begin{array}{c}\text { Theoretical } \\ \text { Plates USP }\end{array} \\ 15.93 & 113.497 & 2147.173 & 47.20 & & 1.1 & 16185 \\ 18.65 & 97.101 & 2402.204 & 52.80 & 4.8 & 0.9 & 13813\end{array}$

HPLC spectra of (R)- 5

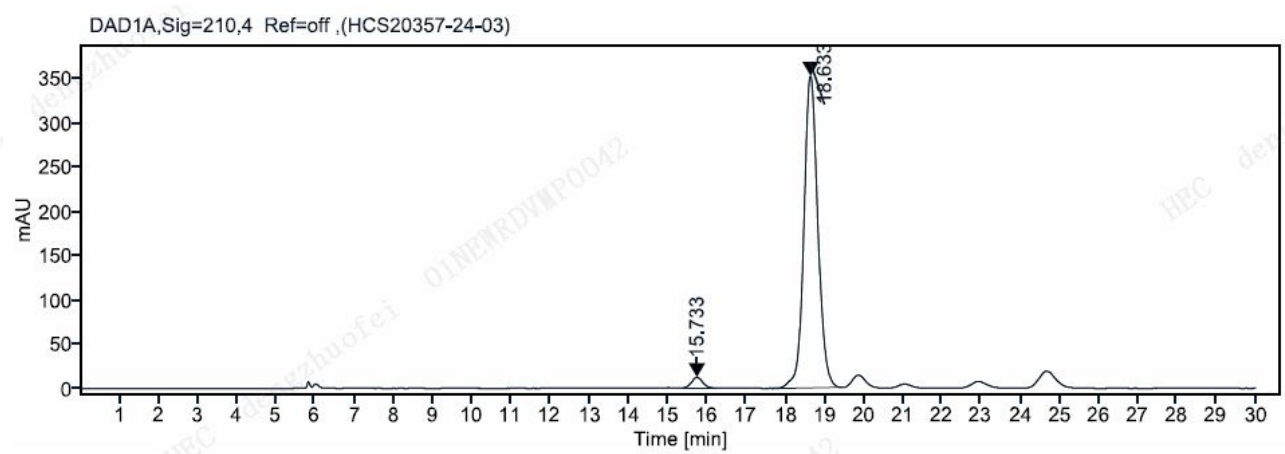

Signal: $\quad$ DAD1A, Sig $=210,4$ Ref $=$ off

$\begin{array}{ccccccc}\text { RT [min] } & \text { Height } & \text { Area } & \text { Area \% } & \begin{array}{c}\text { Resolution } \\ \text { USP }\end{array} & \text { Tail } & \begin{array}{c}\text { Theoretical } \\ \text { Plates USP }\end{array} \\ 15.73 & 12.356 & 243.493 & 2.71 & & 1.1 & 14429 \\ 18.63 & 352.702 & 8738.889 & 97.29 & 5.0 & 1.1 & 13526\end{array}$


HPLC spectra of Rac-6

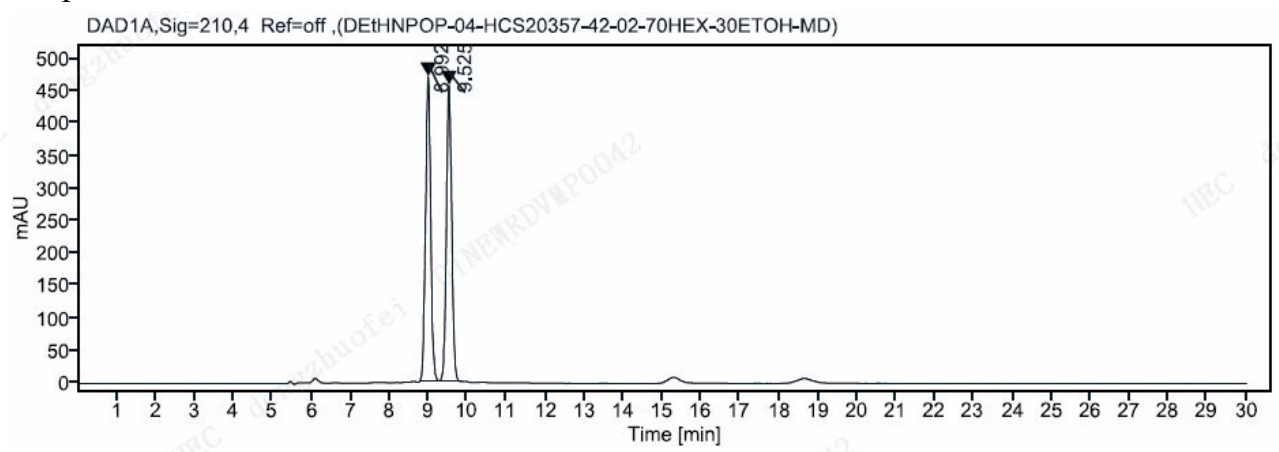

Signal: DAD1A,Sig=210,4 Ref=off

$\begin{array}{ccccccc}\text { RT [min] } & \text { Height } & \text { Area } & \text { Area \% } & \begin{array}{c}\text { Resolution } \\ \text { USP }\end{array} & \text { Tail } & \begin{array}{c}\text { Theoretical } \\ \text { Plates USP }\end{array} \\ 8.99 & 469.923 & 4341.812 & 50.03 & & 1.0 & 21720 \\ 9.52 & 456.858 & 4336.688 & 49.97 & 2.2 & 1.0 & 23174\end{array}$

HPLC spectra of (R)- 6

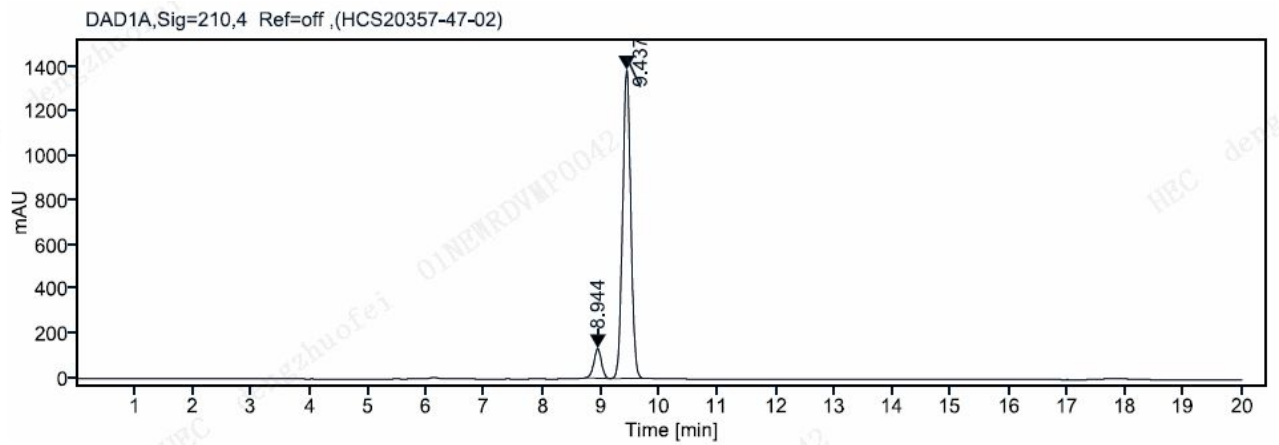

Signal: $\quad$ DAD1A,Sig=210,4 Ref=off

$\begin{array}{ccccccc}\text { RT [min] } & \text { Height } & \text { Area } & \text { Area \% } & \begin{array}{c}\text { Resolution } \\ \text { USP }\end{array} & \text { Tail } & \begin{array}{c}\text { Theoretical } \\ \text { Plates USP }\end{array} \\ 8.94 & 133.563 & 1199.056 & 8.12 & & 0.9 & 22539 \\ 9.44 & 1385.726 & 13564.617 & 91.88 & 2.0 & 1.0 & 21501\end{array}$


HPLC spectra of Rac-7a

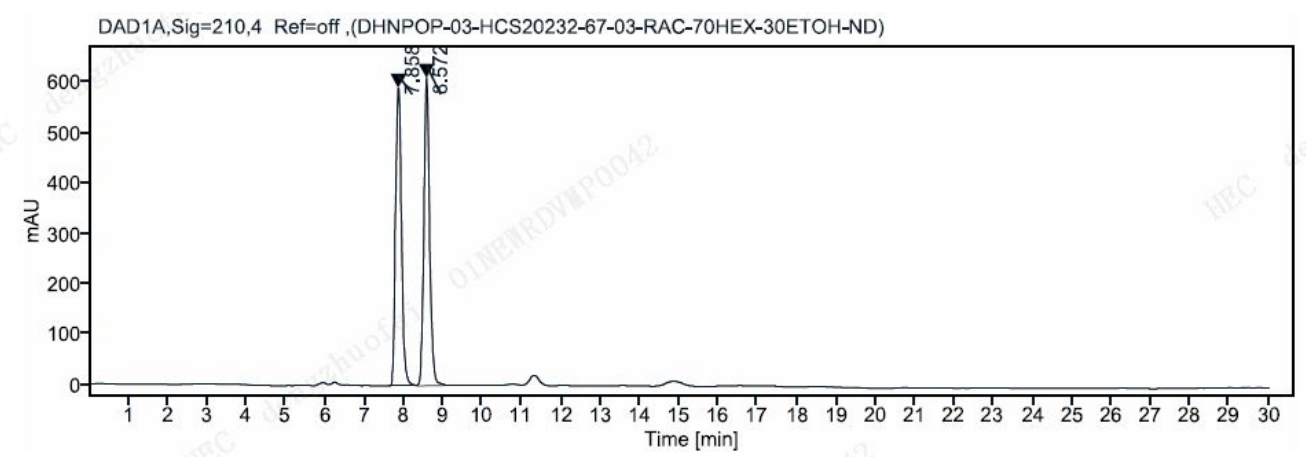

Signal: DAD1A,Sig=210,4 Ref=off

\begin{tabular}{|c|c|c|c|c|c|c|}
\hline RT [min] & Height & Area & Area \% & $\begin{array}{c}\text { Resolution } \\
\text { USP }\end{array}$ & Tail & $\begin{array}{l}\text { Theoretical } \\
\text { Plates USP }\end{array}$ \\
\hline 7.86 & 592.868 & 6204.110 & 49.71 & & 1.1 & 12977 \\
\hline 8.57 & 612.425 & 6276.487 & 50.29 & 2.6 & 1.2 & 16684 \\
\hline
\end{tabular}

HPLC spectra of (R)- 7a

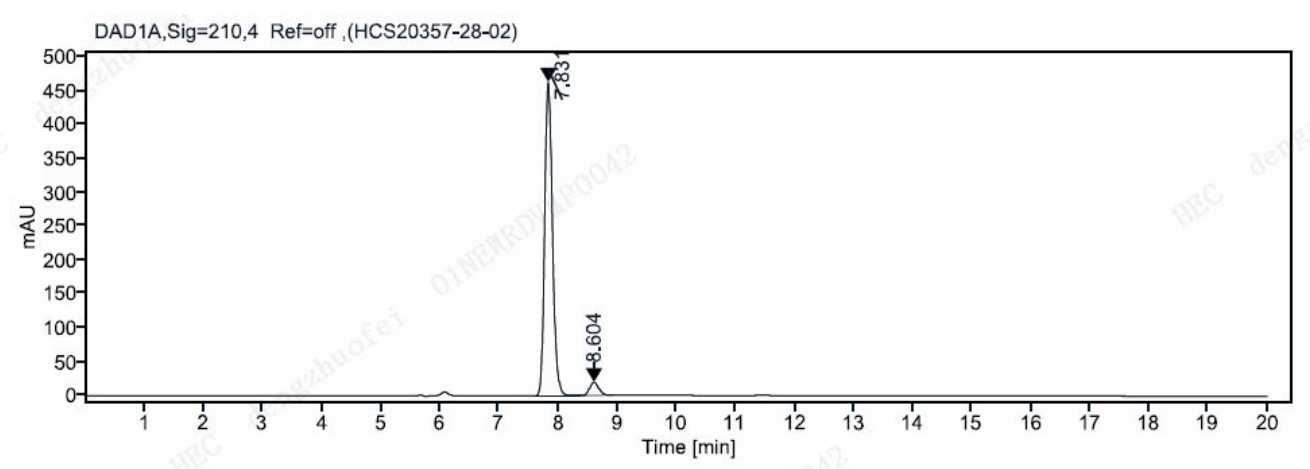

Signal: $\quad$ DAD1A, Sig $=210,4$ Ref $=$ off

$\begin{array}{ccccccc}\text { RT [min] } & \text { Height } & \text { Area } & \text { Area \% } & \begin{array}{c}\text { Resolution } \\ \text { USP }\end{array} & \text { Tail } & \begin{array}{c}\text { Theoretical } \\ \text { Plates USP }\end{array} \\ 7.83 & 462.044 & 4217.559 & 95.13 & & 1.2 & 17238 \\ 8.60 & 19.594 & 216.060 & 4.87 & 2.9 & 1.2 & 14218\end{array}$


HPLC spectra of Rac-7b

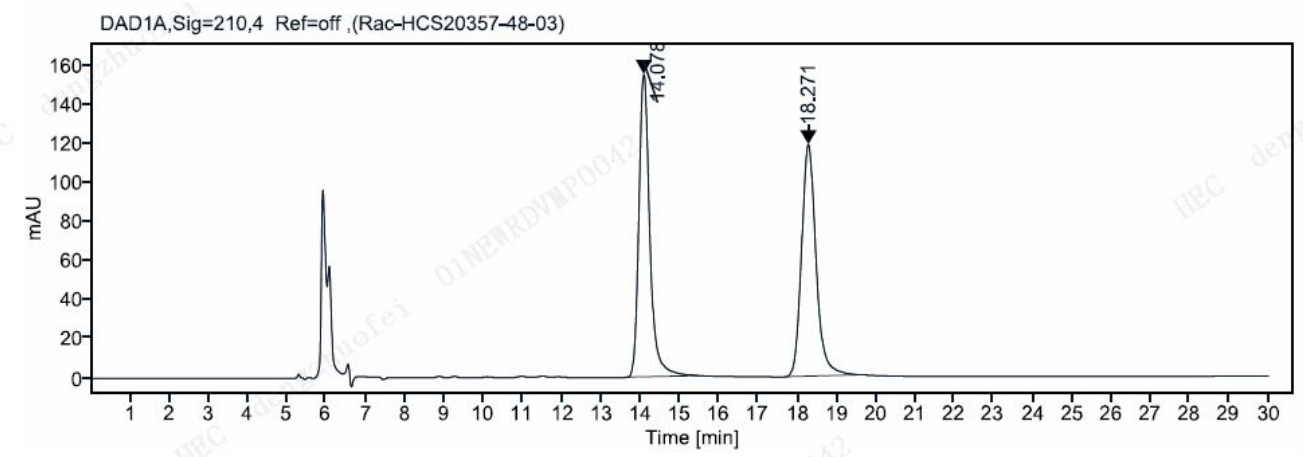

Signal: $\quad D A D 1 A, S i g=210,4$ Ref $=$ off

$\begin{array}{ccccccc}\text { RT [min] } & \text { Height } & \text { Area } & \text { Area \% } & \begin{array}{c}\text { Resolution } \\ \text { USP }\end{array} & \text { Tail } & \begin{array}{c}\text { Theoretical } \\ \text { Plates USP }\end{array} \\ 14.08 & 154.729 & 2997.565 & 49.47 & & 1.3 & 13544 \\ 18.27 & 118.520 & 3062.166 & 50.53 & 7.4 & 1.2 & 12390\end{array}$

HPLC spectra of (R)-7b

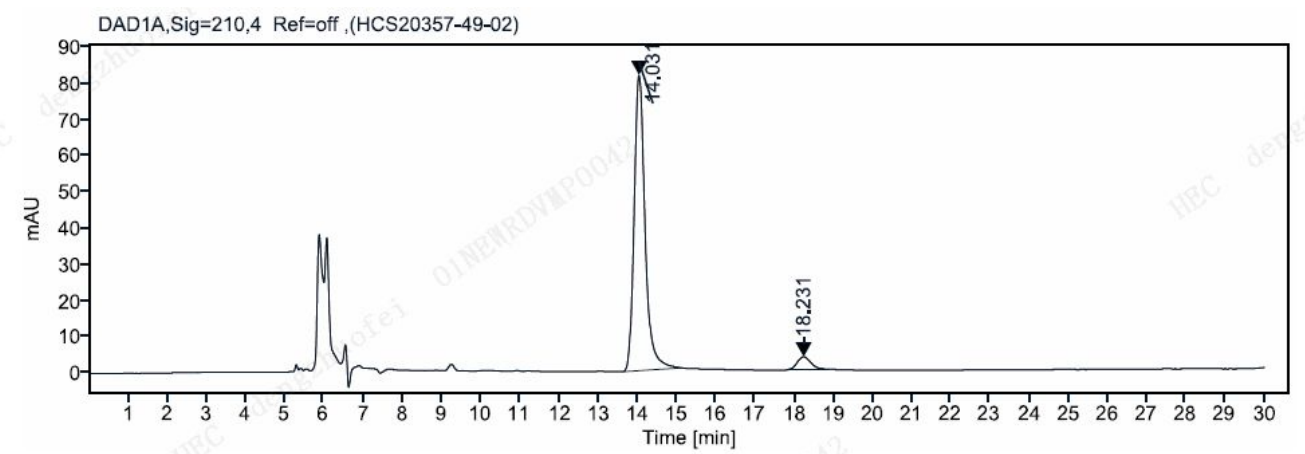

Signal: $\quad$ DAD1A,Sig $=210,4$ Ref $=$ off

$\begin{array}{ccccccc}\text { RT [min] } & \text { Height } & \text { Area } & \text { Area \% } & \begin{array}{c}\text { Resolution } \\ \text { USP }\end{array} & \text { Tail } & \begin{array}{c}\text { Theoretical } \\ \text { Plates USP }\end{array} \\ 14.03 & 81.640 & 1576.447 & 94.95 & & 1.3 & 13341 \\ 18.23 & 3.540 & 83.881 & 5.05 & 7.5 & 1.2 & 13383\end{array}$


HPLC spectra of Rac-8

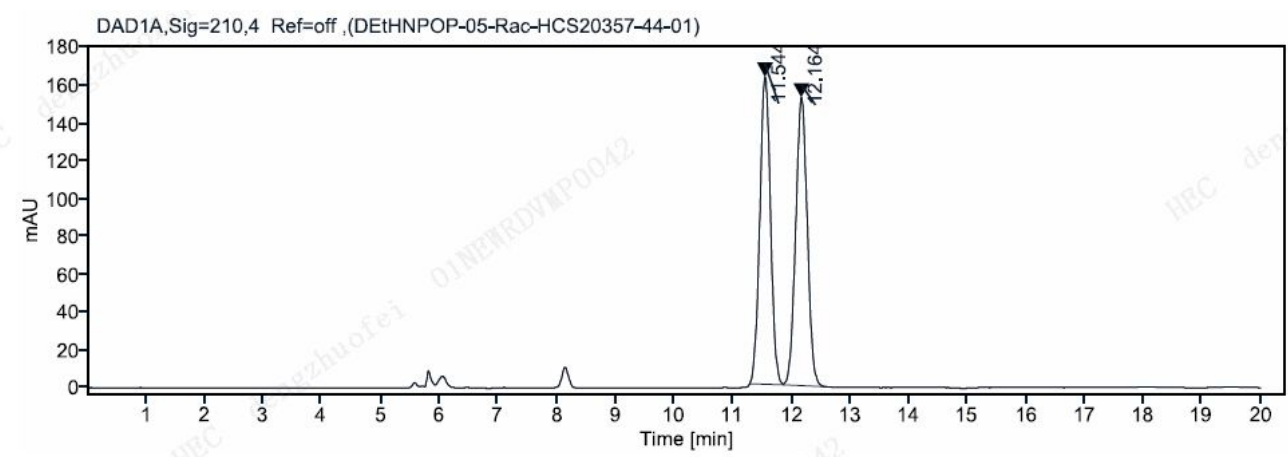

Signal: $\quad$ DAD1A, Sig $=210,4$ Ref $=$ off

$\begin{array}{ccccccc}\text { RT [min] } & \text { Height } & \text { Area } & \text { Area \% } & \begin{array}{c}\text { Resolution } \\ \text { USP }\end{array} & \text { Tail } & \begin{array}{c}\text { Theoretical } \\ \text { Plates USP }\end{array} \\ 11.54 & 162.819 & 2068.072 & 49.59 & & 1.0 & 18675 \\ 12.16 & 152.702 & 2101.936 & 50.41 & 1.8 & 1.0 & 17702\end{array}$

HPLC spectra of (R)-8

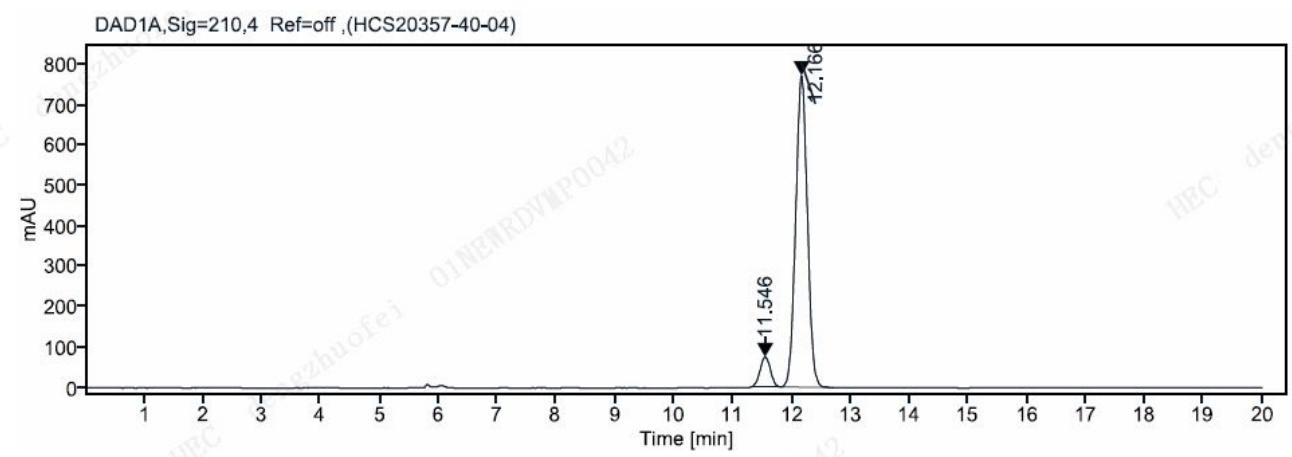

Signal: $\quad$ DAD1A, Sig $=210,4$ Ref $=$ off

$\begin{array}{ccccccc}\text { RT [min] } & \text { Height } & \text { Area } & \text { Area \% } & \begin{array}{c}\text { Resolution } \\ \text { USP }\end{array} & \text { Tail } & \begin{array}{c}\text { Theoretical } \\ \text { Plates USP }\end{array} \\ 11.55 & 74.033 & 912.965 & 7.89 & & 1.0 & 19344 \\ 12.17 & 770.657 & 10651.173 & 92.11 & 1.8 & 1.0 & 17569\end{array}$


HPLC spectra of Rac-9

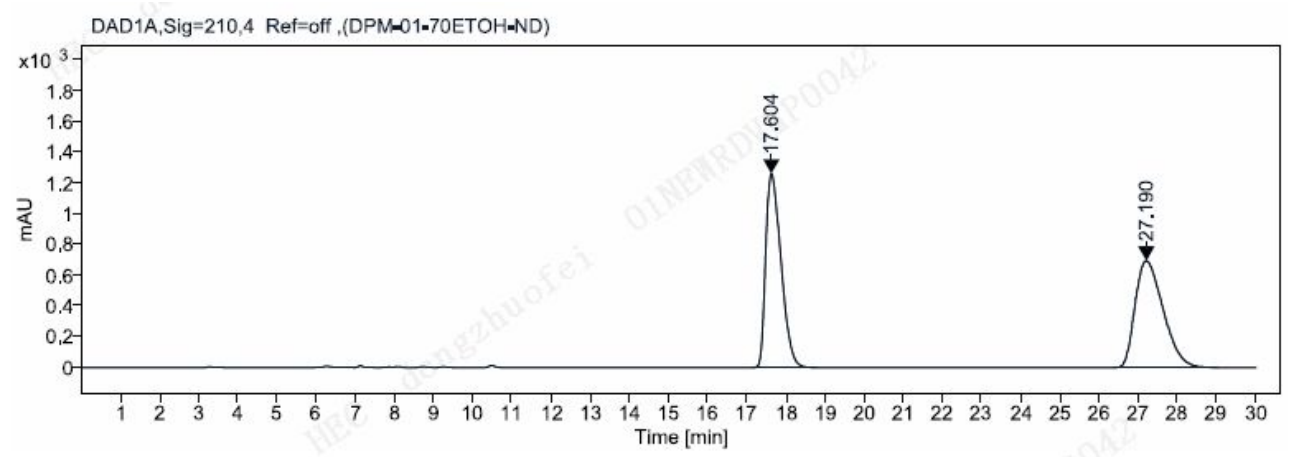

Signal: DAD1A, Sig $=210,4$ Ref $=$ off

$\begin{array}{ccccccc}\text { RT [min] } & \text { Height } & \text { Area } & \text { Area \% } & \begin{array}{c}\text { Resolution } \\ \text { USP }\end{array} & \text { Tail } & \begin{array}{c}\text { Theoretical } \\ \text { Plates USP }\end{array} \\ 17.60 & 1262.778 & 34315.834 & 49.79 & & 1.5 & 9170 \\ 27.19 & 695.519 & 34611.701 & 50.21 & 9.2 & 1.4 & 6556\end{array}$

HPLC spectra of chiral-9

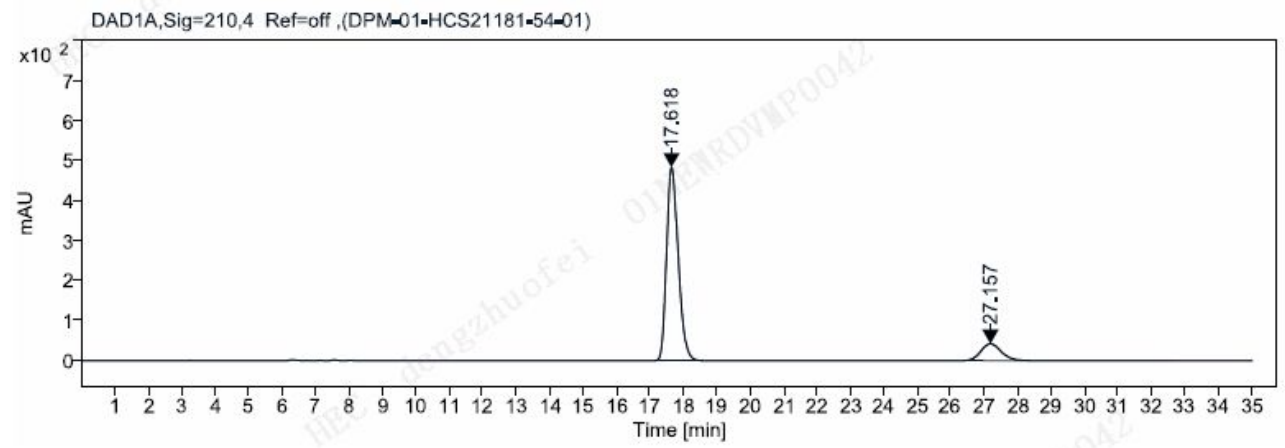

Signal: $\quad$ DAD1A,Sig $=210,4$ Ref $=$ off

$\begin{array}{ccccccc}\text { RT [min] } & \text { Height } & \text { Area } & \text { Area } \% & \begin{array}{c}\text { Resolution } \\ \text { USP }\end{array} & \text { Tail } & \begin{array}{c}\text { Theoretical } \\ \text { Plates USP }\end{array} \\ 17.62 & 484.079 & 11765.422 & 86.90 & & 1.3 & 12009 \\ 27.16 & 42.118 & 1774.126 & 13.10 & 10.8 & 1.2 & 9353\end{array}$

